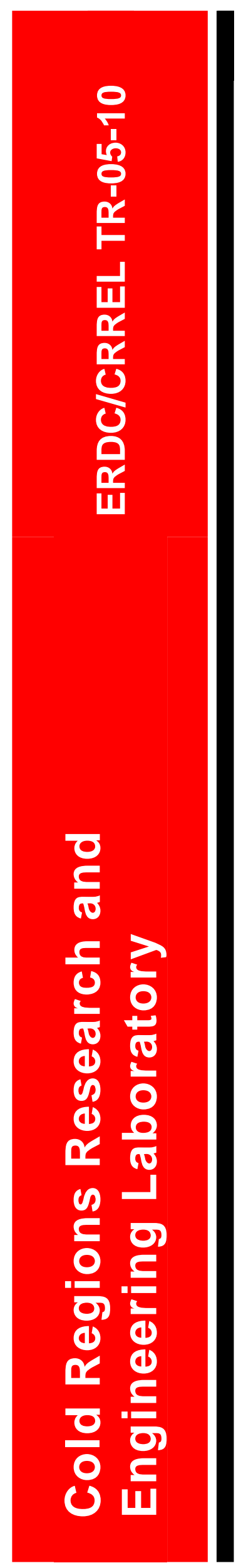

\title{
Short-range Seismic and Acoustic Signature Measurements Through Forest
}

Stephen N. Decato, Donald G. Albert,

Frank E. Perron Jr., and David L. Carbee

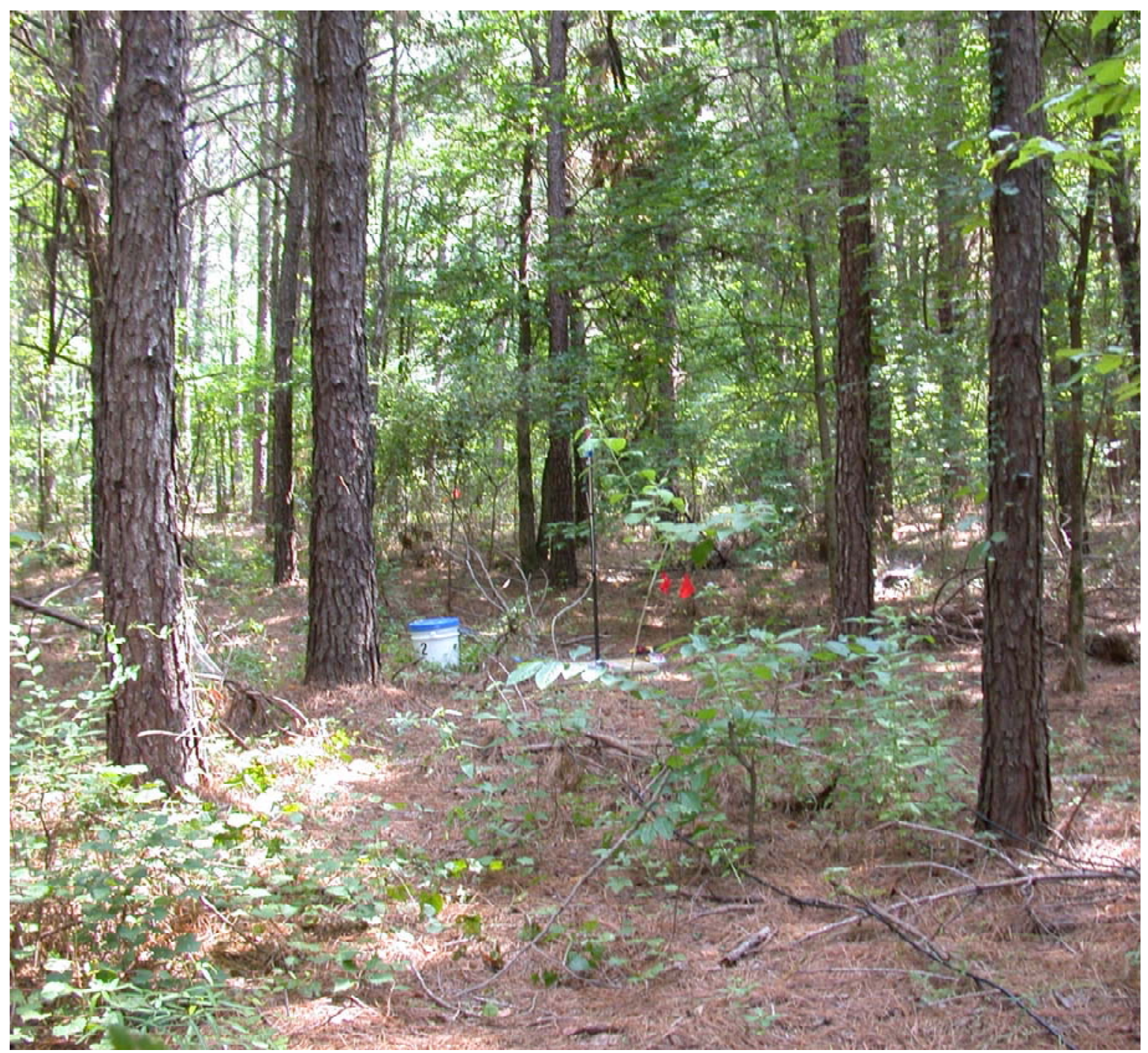

Approved for public release; distribution is unlimited. 



\section{Short-range Seismic and Acoustic Signature Measurements Through Forest}

Stephen N. Decato, Donald G. Albert, Frank E. Perron Jr., and David L. Carbee

U.S. Army Engineer Research and Development Center Cold Regions Research and Engineering Laboratory

72 Lyme Road

Hanover NH 03755-1290

Approved for public release; distribution is unlimited 
ABSTRACT: The effect of forests on low frequency military noise propagation is unknown. As part of a joint project, ERDC-CERL and ERDC CRREL conducted measurements at the Lone Star Army Ammunition Plant located in Texarkana, Texas, to investigate these effects. In this report, the short-range measurements conducted by ERDC-CRREL are documented. Blast noise waveforms produced by C4 explosions at distances from 30 to $567 \mathrm{~m}$ were recorded and are presented in this report. In all, 42 different explosions were recorded, producing 314 high quality pressure waveforms for analysis. Additional reports documenting the long-range measurements and analyzing the recorded data are in preparation.

DISCLAIMER: The contents of this report are not to be used for advertising, publication, or promotional purposes. Citation of trade names does not constitute an official endorsement or approval of the use of such commercial products. All product names and trademarks cited are the property of their respective owners. The findings of this report are not to be construed as an official Department of the Army position unless so designated by other authorized documents. 


\section{CONTENTS}

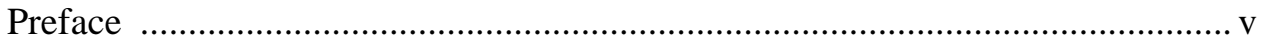

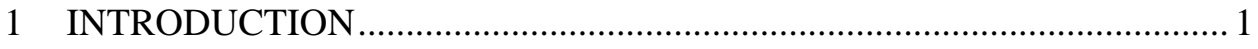

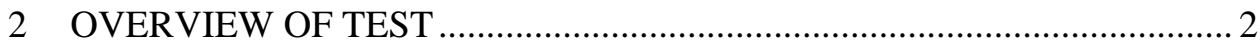

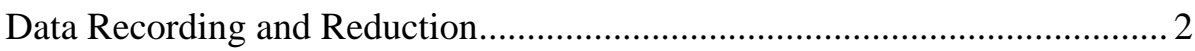

List of Signature Records ........................................................................... 5

Specifications for Sources used in Measurements ........................................ 6

3 CRREL SENSOR CONFIGURATION ...................................................... 9

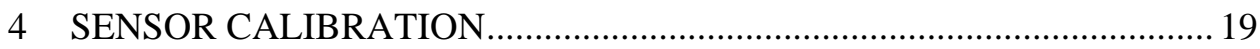

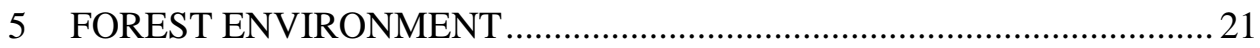

6 TEXAS 2002 TIME BREAK CORRECTIONS AND ACOUSTIC

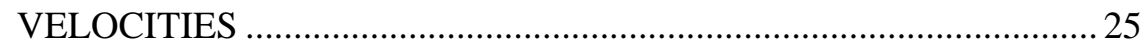

7 SIGNATURE RECORDINGS AND DATA QUALITY .............................. 28

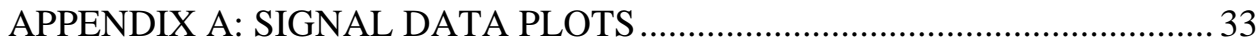

APPENDIX B: MATLAB PROGRAMS TO READ AND PLOT BINARY DATA FROM NZ SEISMOGRAPH AND TO WRITE MULTICHANNEL ASCII DATA FILES............................................. 90

APPENDIX C: MATLAB PROGRAMS TO READ AND PLOT MULTICHANNEL ASCII DATA FILES........................................... 118

\section{ILLUSTRATIONS}

Figure 1. View of the Lone Star AAP test range, with approximate locations of

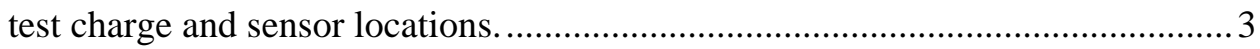

Figure 2. Test Charge Site 2 (TC 2) and a sketch of the test array....................... 4 Figure 3. Geometrics NZ Digital Seismograph, set up inside a vehicle parked behind a protection soil berm......................................................................... 5

Figure 4. .45-caliber calibration pistol firing blanks at a 1-m height.................... 7

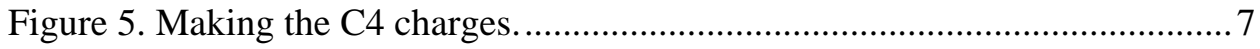

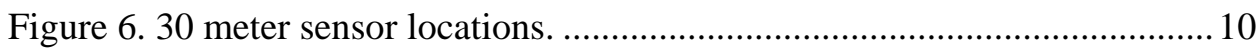

Figure 7. 60 meter sensor locations. .......................................................... 11

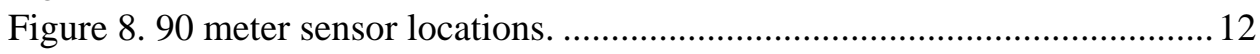




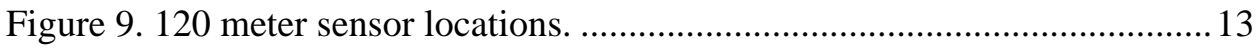

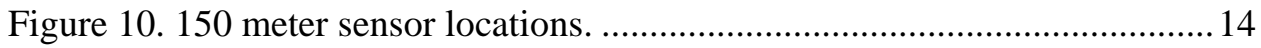

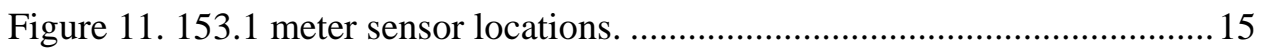

Figure 12. CRREL munitions demolition array ................................................. 16

Figure 13. Typical sensor calibration............................................................. 19

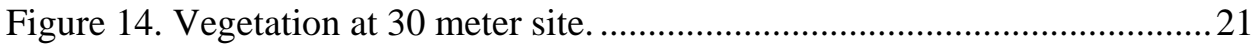

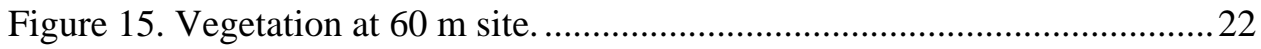

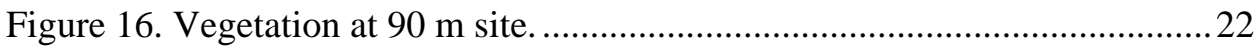

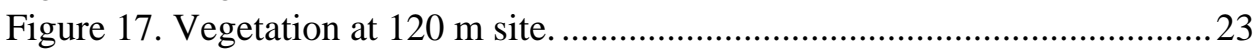

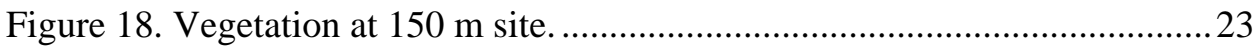

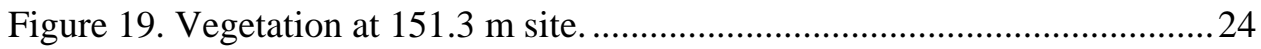

\section{TABLES}

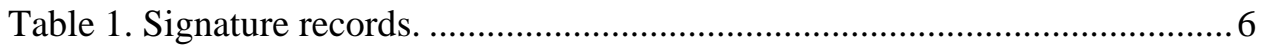

Table 2. Distances between the CRREL and CERL locations.............................. 9

Table 3. Wave propagation sensor array........................................................ 17

Table 4. Changes to wave propagation sensor array.......................................... 18

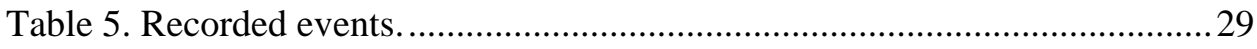

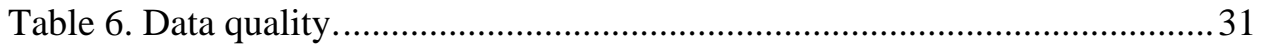




\section{PREFACE}

This report was prepared by Stephen N. Decato, Physical Science Technician, Donald G. Albert, Research Geophysicist, Frank E. Perron Jr., Physical Science Technician, and David L. Carbee, Physical Science Technician, Geophysical Sciences Branch, U.S. Army Engineer Research and Development Center, Cold Regions Research and Engineering Laboratory.

In recent years the increase of civilian populations living on the outskirts of U.S. Army installations has led to more frequent noise complaints. While the Army normally uses forest management techniques to ensure the health of the forests on its installations, a question that has arisen is whether these forest practices, including tree harvesting, might adversely impact the noise problem by reducing the noise absorption characteristics of the forested land. Underlying this question is the assumption that forests absorb noise. While this is certainly true at high frequencies (in the $\mathrm{kHz}$ band), little work has been done at the lower frequencies (below $100 \mathrm{~Hz}$ ) often produced by Army demolition and training activities. As a result of this lack of knowledge, ERDC, under the direction of Dr. Larry Pater, ERDC-CERL, is embarking on a study of the low frequency noise attenuation by forests. As part of this study, a joint measurement program involving two ERDC Laboratories, CERL and CRREL, was conducted at the Lone Star Army Ammunition Plant in Texarkana, Texas. The purpose of this report is to document the CRREL measurements. Additional reports will document the CERL measurements and analyze the experimental data to determine the effect of the forest on low frequency military blast noise.

Field personnel during this test included Don Albert, Steve Decato, and Frank Perron from CRREL, and Larry Pater, Mike White, Richard Racioppi, Jeff Mifflin, George Swenson, Brent Miller, and Achal Modi from CERL. The authors especially thank Dave Self, Jesse Stewart, and all the other Lone Star Army Ammunition Plant personnel for their assistance during these measurements. They thank Tom Vorac, U.S. AMC Installations and Security Activity (now with the U.S. Army Environmental Center, Natural Resources), and Dr. Larry Pater, U.S.A ERDC-CERL, for funding this research. Finally, they thank Dr. Pater, Project Leader for Military Noise Management, for the invitation to participate in this project.

This report was prepared under the general supervision of Dr. Richard Detsch, Chief, Geophysical Sciences Branch; and James Wuebben, Acting Director, CRREL.

The Commander of the Engineer Research and Development Center is COL James R. Rowan, EN. The Director is Dr. James R. Houston. 



\title{
Short-range Seismic and Acoustic Signature Measurements Through Forest
}

\author{
STEPHEN N. DECATO, DONALD G. ALBERT, FRANK E. PERRON JR., AND \\ DAVID L. CARBEE
}

\section{INTRODUCTION}

This report documents the measurements and presents the signals recorded during the Blast Noise Propagation Through Forest Test conducted by the U.S. Army ERDC Construction Engineering Research Laboratory and the U.S. Army ERDC Cold Regions Research and Engineering Laboratory. The measurements were conducted at the Lone Star Army Ammunition Plant in Texarkana, Texas, from 23 July through 25 July 2002 to determine the properties of blast noise wave propagation through a forested area. This report contains the recordings made by CRREL; the CERL recordings will be published in a separate report. Use of this and other data sets will assist the Army in determining methods of improving blast noise attenuation in populated areas and enhance forest management techniques.

The blast waves were produced by detonating charges of M112 C4 explosive at various heights and locations. Sledge hammer blows on an aluminum plate and .45 caliber blank rounds were also recorded to provide additional ground and air characterization. Pressure sensors, microphones, and geophones were configured in a straight-line array on the ground and $1.5 \mathrm{~m}$ above the ground surface.

The intent of this report is to provide documentation of the CRREL measurements that will accompany the transmittal of the recorded data on CDROM to the test participants and sponsor. This report includes a list of the records obtained during the tests, documentation of the sensor array, a table of sensors used, environmental characterization, and a description of the data reduction. Plots of all the signatures are provided, and the signatures themselves are included on a CD-ROM in ASCII format. An analysis report of the signatures shown here will be published separately. 


\section{OVERVIEW OF TEST}

The Lone Star Army Ammunition plant routinely detonates obsolete munitions as a method of disposal. On a typical day, as many as 48 shots, each consisting of about $95 \mathrm{lb}$ (43 kg) of explosives, may be detonated. Although these charges are buried 10 to $15 \mathrm{ft}$ ( 3 to $5 \mathrm{~m}$ ) deep in a sandy/silty soil, the shots always "blow out," sending debris high into the air producing a loud blast wave. In recent years, private homes are being built close to the AAP and other Army facilities' boundary lines. This has resulted in concerns about noise complaints from new residents. As the current demolition area at the AAP is surrounded by forest, a scientifically unanswered question is what is the effect of this forest on the noise produced by the explosions? If the trees are cut or thinned as a part of normal forest management plan, will the noise complaints increase? To answer this question, the measurements in this report were conducted.

The measurements were conducted at the (new) Demolition Range at the Lone Star AAP. Figure 1 shows an aerial photograph of the test site with the shot locations (marked TC = Test Charge) and sensor locations. The CRREL sensor array was located in the forest south of TC 3 . Figure 2 shows a schematic of the CRREL sensor locations and a typical test charge (C-4) shot. Details of the sensor array are given in Section 3 of this report.

\section{Data Recording and Reduction}

A Geometrics NZ digital seismograph (Fig. 3) was used to record the signature data from the sensor arrays. We used an $8-\mathrm{kHz}$ sampling rate $(0.125 \mathrm{~ms})$ per channel, providing a 3-dB recording bandwidth of $1.75 \mathrm{~Hz}$ to $3.3 \mathrm{kHz}$. Twentyfour sensor channels were recorded for each test charge.

Because of a malfunction with the CRREL blaster box, the actual shot instant could not be recorded and the recorder could not be automatically started as we normally do. Instead, the recordings were started manually and the shot instant was determined afterwards from the blast wave arrival times. This correction procedure is discussed in Section 6.

The seismograph produces binary data files in an industry standard format called SEG-2. These binary files contain the raw voltage output of the sensors, but contain no other information. We converted these binary files into ASCII files using the MATLAB program listed in Appendix B. The ASCII files have the correct physical units, time information, and sensor location details. The signature plots presented in Appendix A were produced by the MATLAB program 
listed in Appendix C from the ASCII data files. Additional data analysis will be performed using only the ASCII data files.

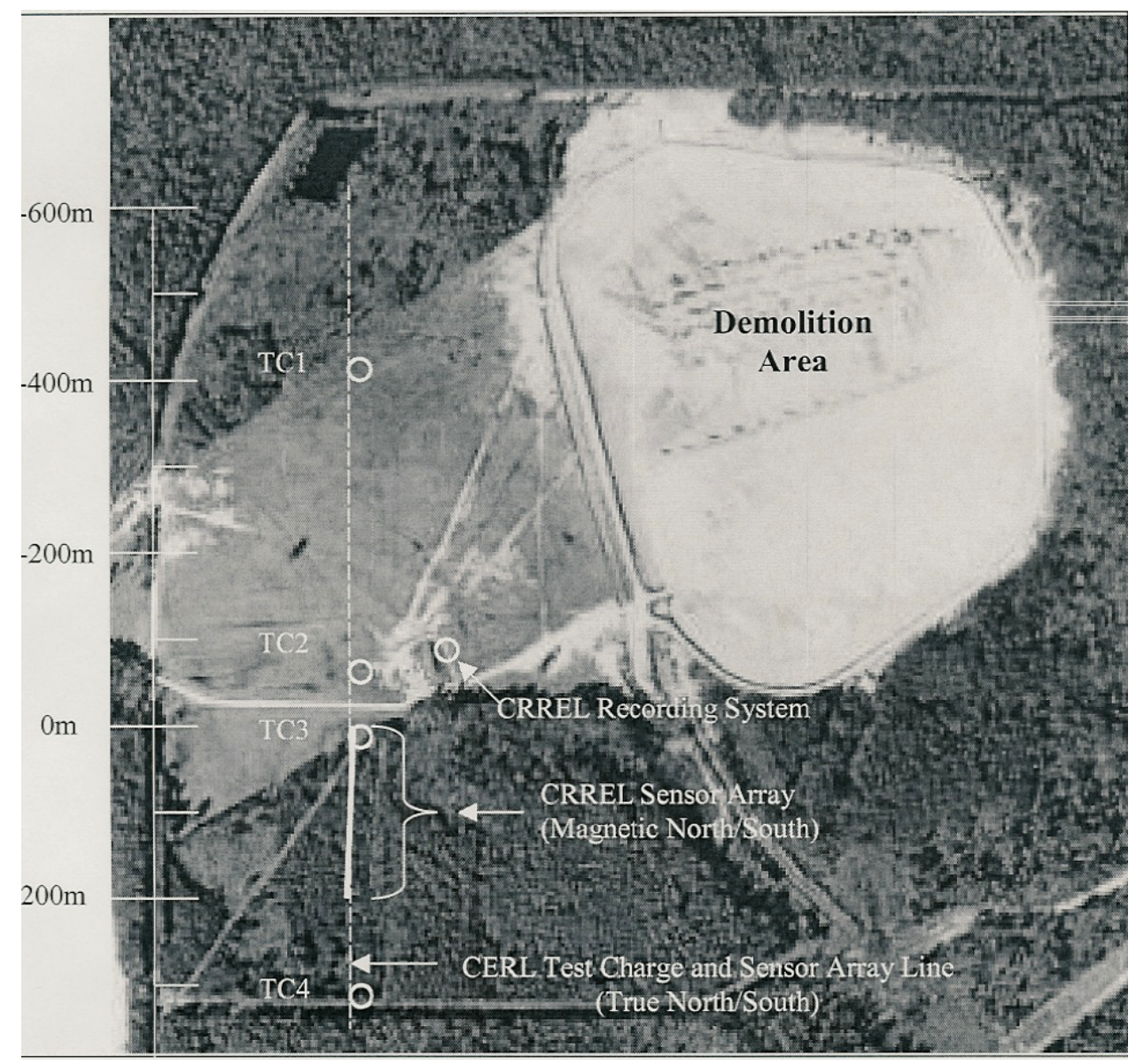

Figure 1. View of the Lone Star AAP test range, with approximate locations of test charge and sensor locations. 

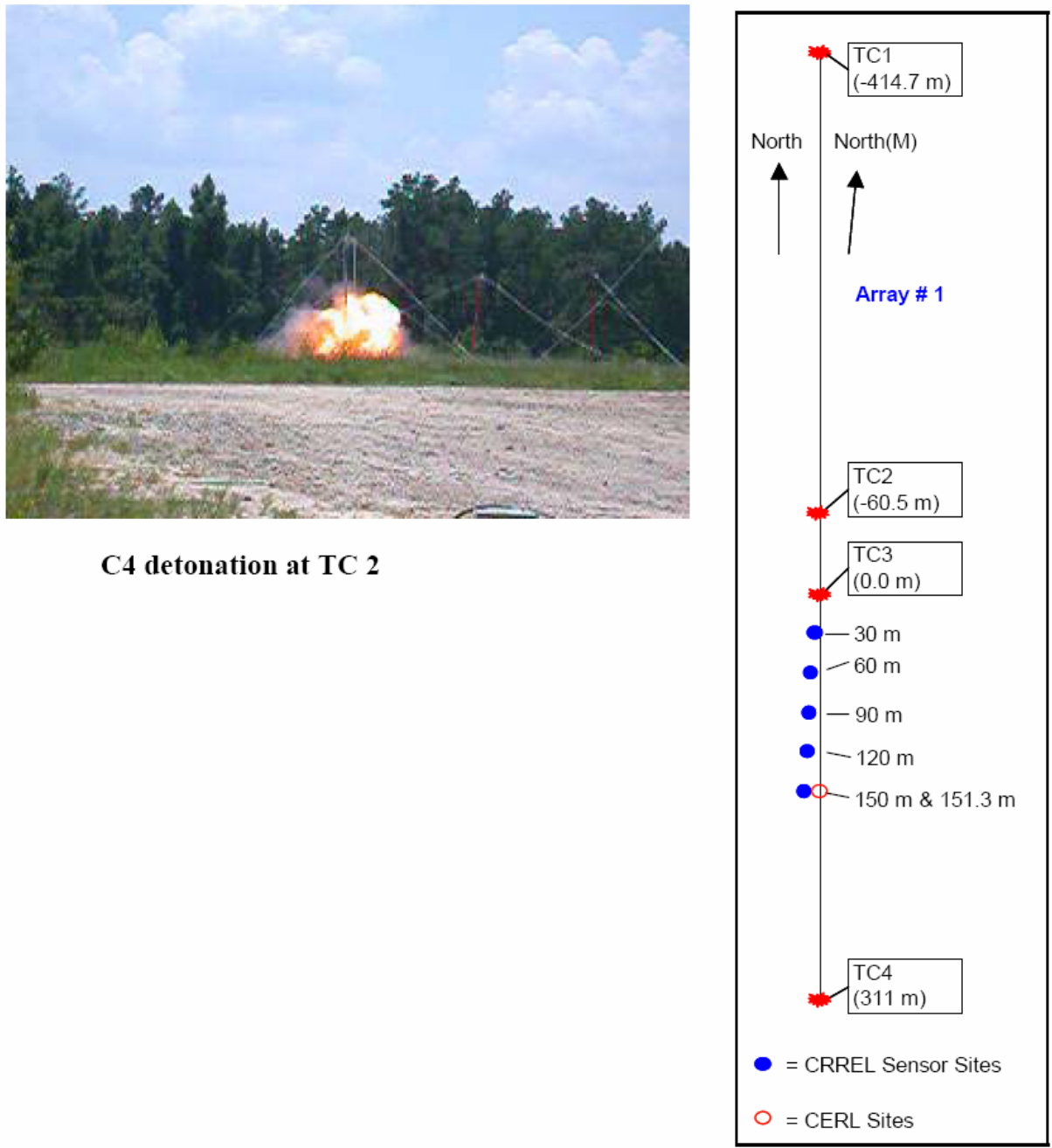

Figure 2. Test Charge Site 2 (TC 2) and a sketch of the test array. 


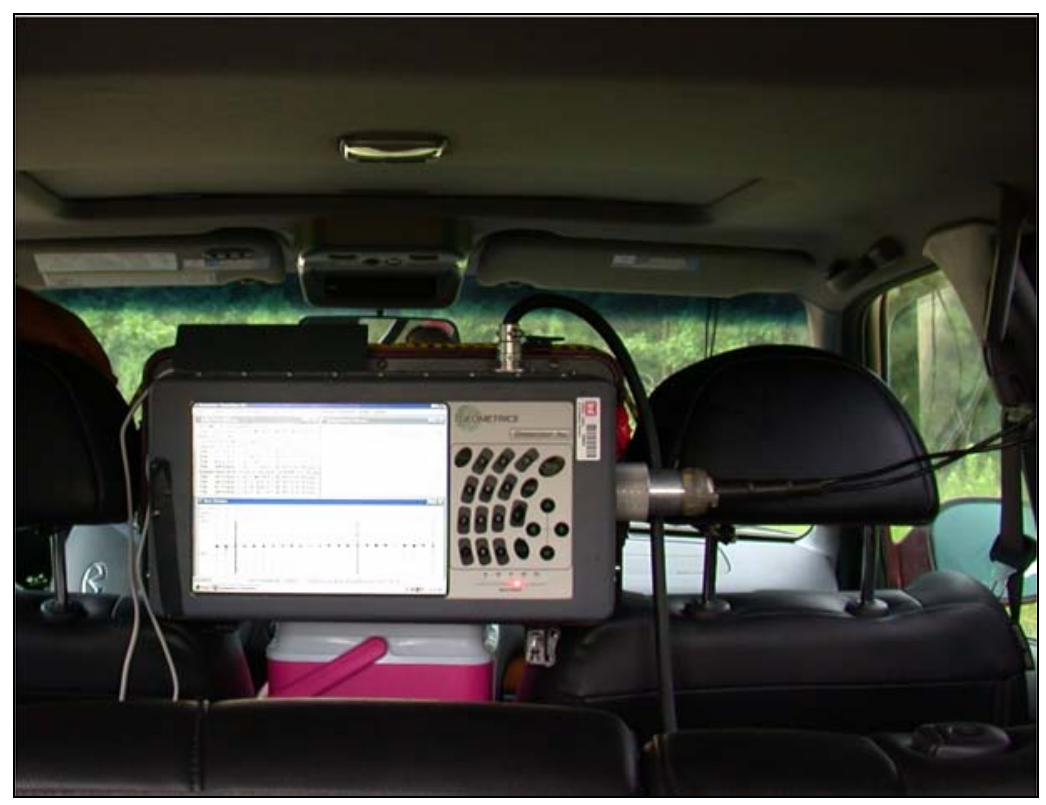

Figure 3. Geometrics NZ Digital Seismograph, set up inside a vehicle parked behind a protection soil berm.

\section{List of Signature Records}

The measurements were conducted without interfering with the normal demolition operations. This meant that the forest testing was delayed and personnel were evacuated from the test site when demolition shots were fired. While we did take advantage of this opportunity to record some of these demolition shots using a separate small sensor array located in the safety zone, those recordings are not of client interest to this study and are not included in this report. They do, however, affect the numbering of the records and files recorded by the digital seismograph.

The following list contains the types of data recorded for the Blast Noise Propagation Test and is included on the CD. NZ file names like "1.dat" through "199.dat" have been converted to multichannel ASCII files with the correct physical units. File names like "TX02002.asc" through “TX02046.asc" are files numbered so that they correspond to CERL's C4 shot numbers. Munition demolition records have been omitted from this report. Table 1 contains the list of signature records. Figures 4 and 5 show our C4 charges and .45-calibre pistol. 
Table 1. Signature records.

\begin{tabular}{|ll|}
\hline 1.dat - 19.dat & .45 cal. blank \\
\hline 20.dat -27. dat & Microphone calibration \\
\hline 28.dat - 38.dat & Demolition of munitions \\
\hline 39.dat - 44.dat & C4 \\
\hline 45.dat & Noise Record \\
\hline 46.dat - 56.dat & Demolition of munitions \\
\hline 57.dat - 58.dat & Noise Record \\
\hline 59.dat & Demolition of munitions \\
\hline 60.dat - 68.dat & C4 \\
\hline 69.dat & Noise Record \\
\hline 70.dat $-74 . d a t$ & C4 \\
\hline 75.dat $-81 . d a t$ & Demolition of munitions \\
\hline 82.dat - 91.dat & Calibration \\
\hline 92.dat & Noise Record \\
\hline 93.dat - 113.dat & C4 \\
\hline 114.dat - 199.dat & Calibration \\
\hline
\end{tabular}

\section{Specifications for Sources used in Measurements}

\begin{tabular}{|c|c|c|c|}
\hline \multirow[t]{7}{*}{ C4 } & $1 / 2$ brick & \multicolumn{2}{|c|}{$0.63 \mathrm{lb}(0.28 \mathrm{~kg})$} \\
\hline & 1 brick & \multicolumn{2}{|c|}{$1.25 \mathrm{lb}(0.57 \mathrm{~kg})$} \\
\hline & 2 bricks & \multicolumn{2}{|c|}{$2.50 \mathrm{lb}(1.23 \mathrm{~kg})$} \\
\hline & 4 bricks & \multicolumn{2}{|c|}{$5.00 \mathrm{lb}(2.27 \mathrm{~kg})$} \\
\hline & 5 bricks & \multicolumn{2}{|c|}{$6.25 \mathrm{lb}(2.84 \mathrm{~kg})$} \\
\hline & 8 bricks & \multicolumn{2}{|c|}{$10.00 \mathrm{lb}(4.55 \mathrm{~kg})$} \\
\hline & 10 bricks & \multicolumn{2}{|c|}{12.50 lb (5.67 kg) } \\
\hline \multirow[t]{8}{*}{.45 cal blank } & Overall Length & \multicolumn{2}{|c|}{$0.89 \mathrm{in} .(22.6 \mathrm{~mm})$} \\
\hline & Case Length & \multicolumn{2}{|c|}{0.89 in. $(22.6 \mathrm{~mm})$} \\
\hline & \multicolumn{3}{|c|}{ Cartridge Weight 7.6 g } \\
\hline & Case Weight & \multicolumn{2}{|c|}{$5.8 \mathrm{~g}$} \\
\hline & Powder Weight & \multicolumn{2}{|l|}{$31 \mathrm{~g}$} \\
\hline & Powder Type & \multicolumn{2}{|c|}{ Black, FFFFG, CCI } \\
\hline & Primer Type & \multicolumn{2}{|c|}{ Magnum } \\
\hline & Manufacture & \multicolumn{2}{|c|}{ Custom made } \\
\hline \multirow[t]{7}{*}{ 10-lb (4.5-kg) sledge hammer } & Overall Length & \multicolumn{2}{|c|}{34 in. $(86.7 \mathrm{~cm})$} \\
\hline & Overall Weight & \multicolumn{2}{|c|}{$11.68 \mathrm{lb}(5.30 \mathrm{~kg})$} \\
\hline & Head Weight & \multicolumn{2}{|c|}{$10.0 \mathrm{lb}(4.55 \mathrm{~kg})$} \\
\hline & \multicolumn{2}{|c|}{ Strike Plate Material } & 6061T6 Aluminum \\
\hline & \multicolumn{2}{|c|}{ Strike Plate Area } & 103.1 in. $^{2}\left(668 \mathrm{~cm}^{2}\right)$ \\
\hline & \multicolumn{2}{|c|}{ Strike Plate Thickness } & $1 \mathrm{in} .(2.54 \mathrm{~cm})$ \\
\hline & \multicolumn{2}{|c|}{ Strike Plate Weight } & 10.36 lb. $(4.70 \mathrm{~kg})$ \\
\hline
\end{tabular}



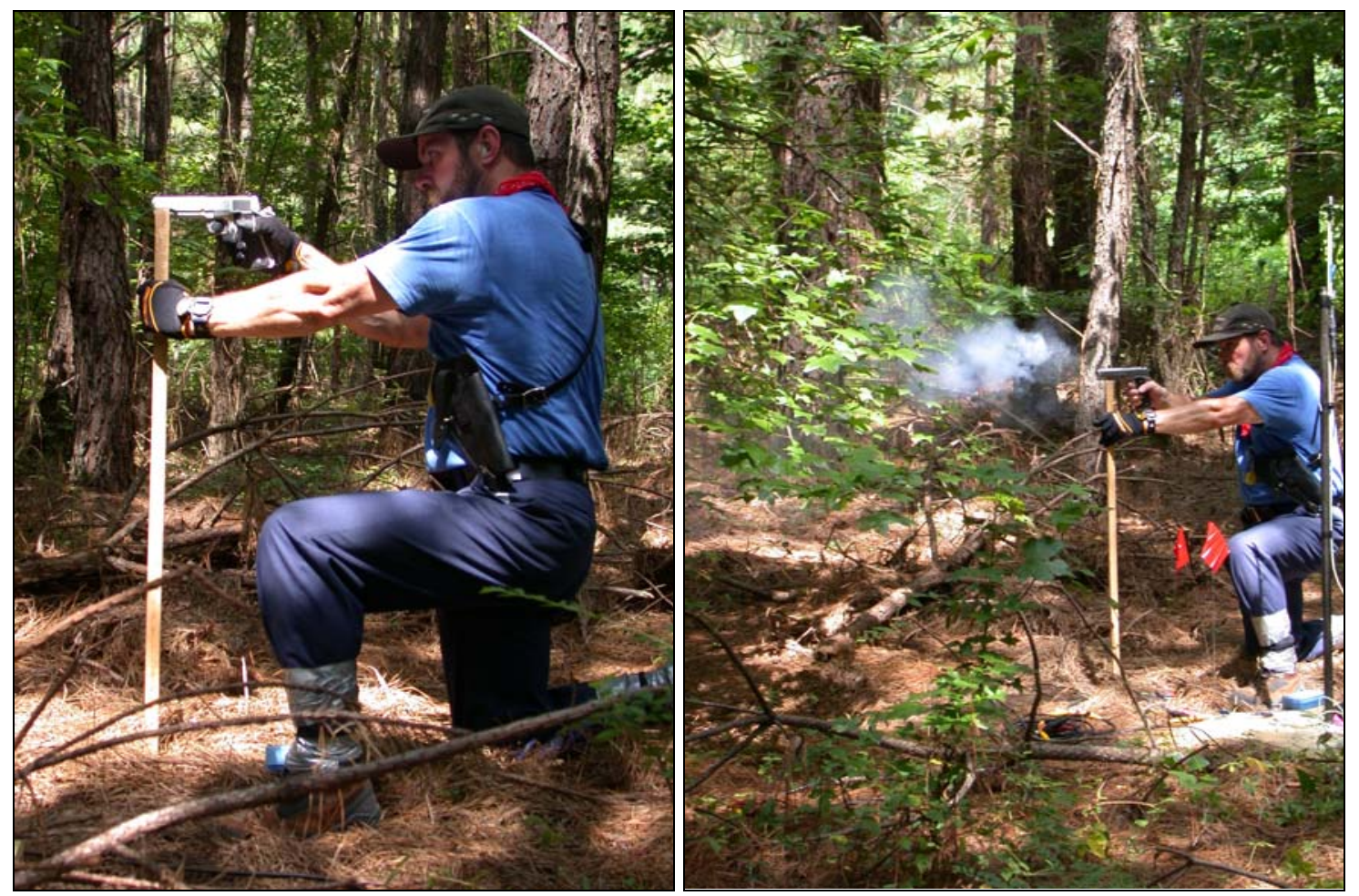

Figure 4. .45-caliber calibration pistol firing blanks at a 1-m height.

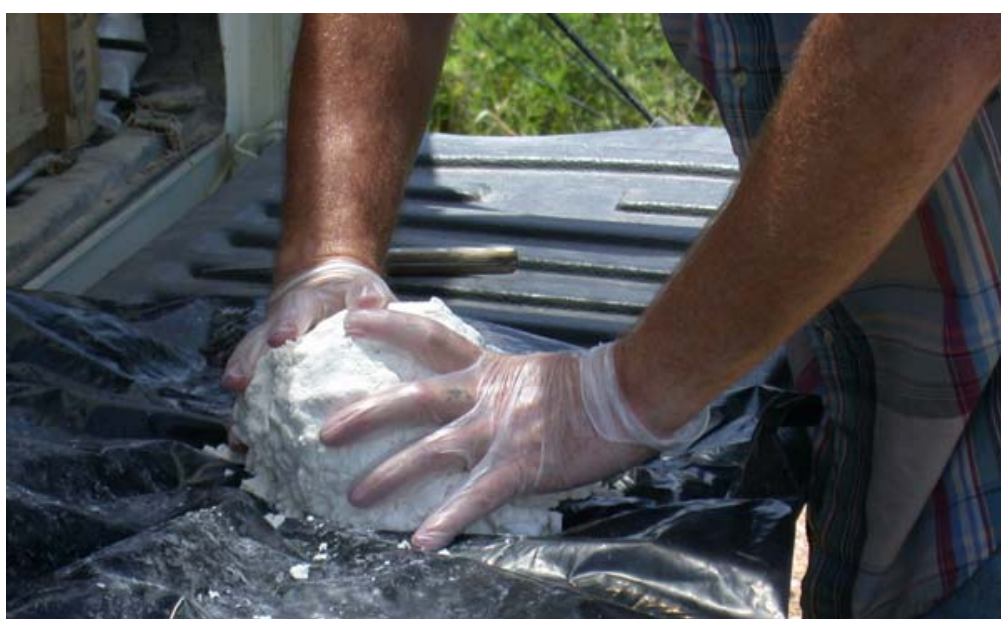

a. C4 being formed into a $5-\mathrm{lb}(2.25-\mathrm{kg})$ spherical charge.

Figure 5. Making the C4 charges. 


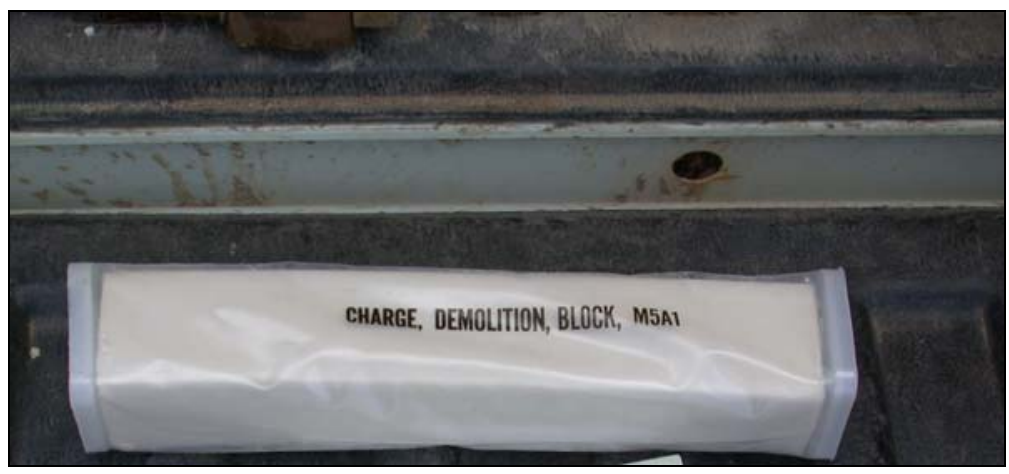

b. A $1.25-\mathrm{lb}(0.68-\mathrm{kg})$ brick of C4.

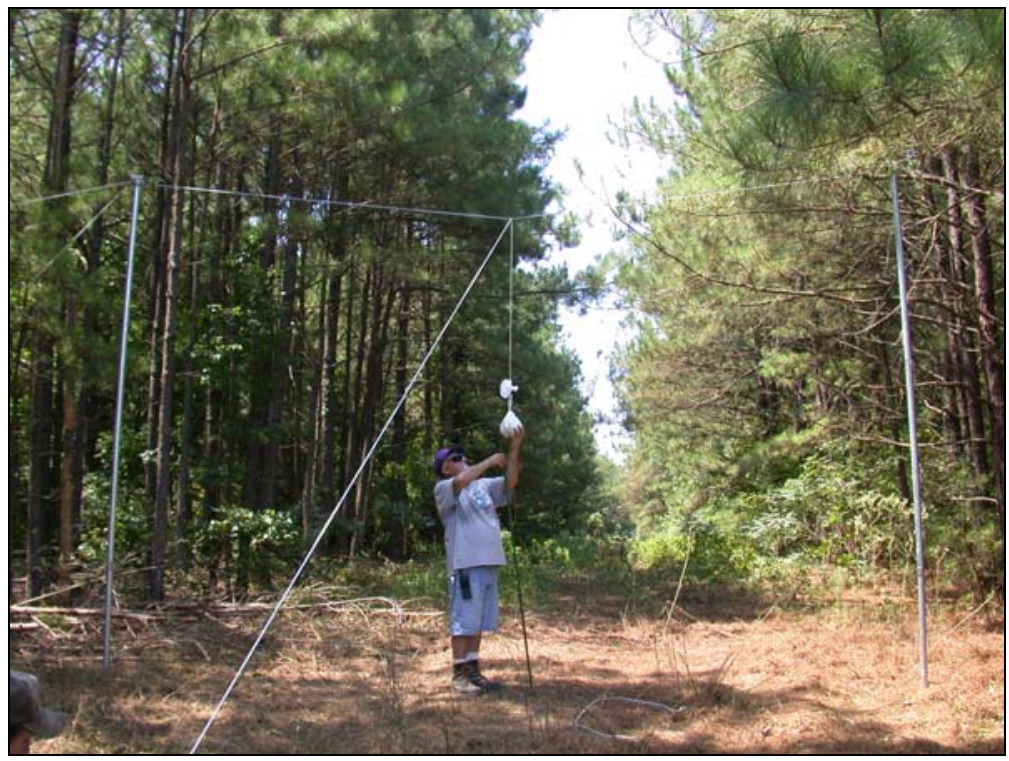

c. C4 spherical charge being suspended at TC 3.

Figure 5 (con't). Making the $\mathrm{C} 4$ charges. 


\section{CRREL SENSOR CONFIGURATION}

The sensor configuration installed by CRREL consisted of a linear array with multi-sensor stations. The array was located in the forest between TC 3 and TC 4 . It extended from 30 to $151.3 \mathrm{~m}$ magnetic south of TC 3 . The goal of this sensor array was to obtain a detailed view of the blast waves as they propagated into the forest.

CERL also installed and recorded signatures from their own independent sensor array. This array was located both inside and outside of the forest on a true north-south line. This array was designed to obtain a complete overview of the blast wave propagation at the site as well as details of the blast wave generation by the source.

Table 2 lists the distances between the CRREL and CERL locations. This difference was due to a 3.5 degree east deviation between true north and magnetic north.

Figures 6 through 11 show the locations of the of the CRREL instrument stations. Table 3 details the sensors used in the CRREL Blast Wave Propagation Array. Table 4 notes any sensor changes made to that array, during the measurements.

Figure 12 shows the CRREL array used to record the large munitions demolition shots. Further discussion of this array is omitted from this report.

Table 2. Distances between the CRREL and CERL locations.

\begin{tabular}{|c|c|}
\hline Location & Distance $(\mathbf{m})$ \\
\hline 30 meters & 1.83 \\
60 meters & 3.66 \\
90 meters & 5.49 \\
120 meters & 7.32 \\
150 meters & 9.15 \\
\hline
\end{tabular}



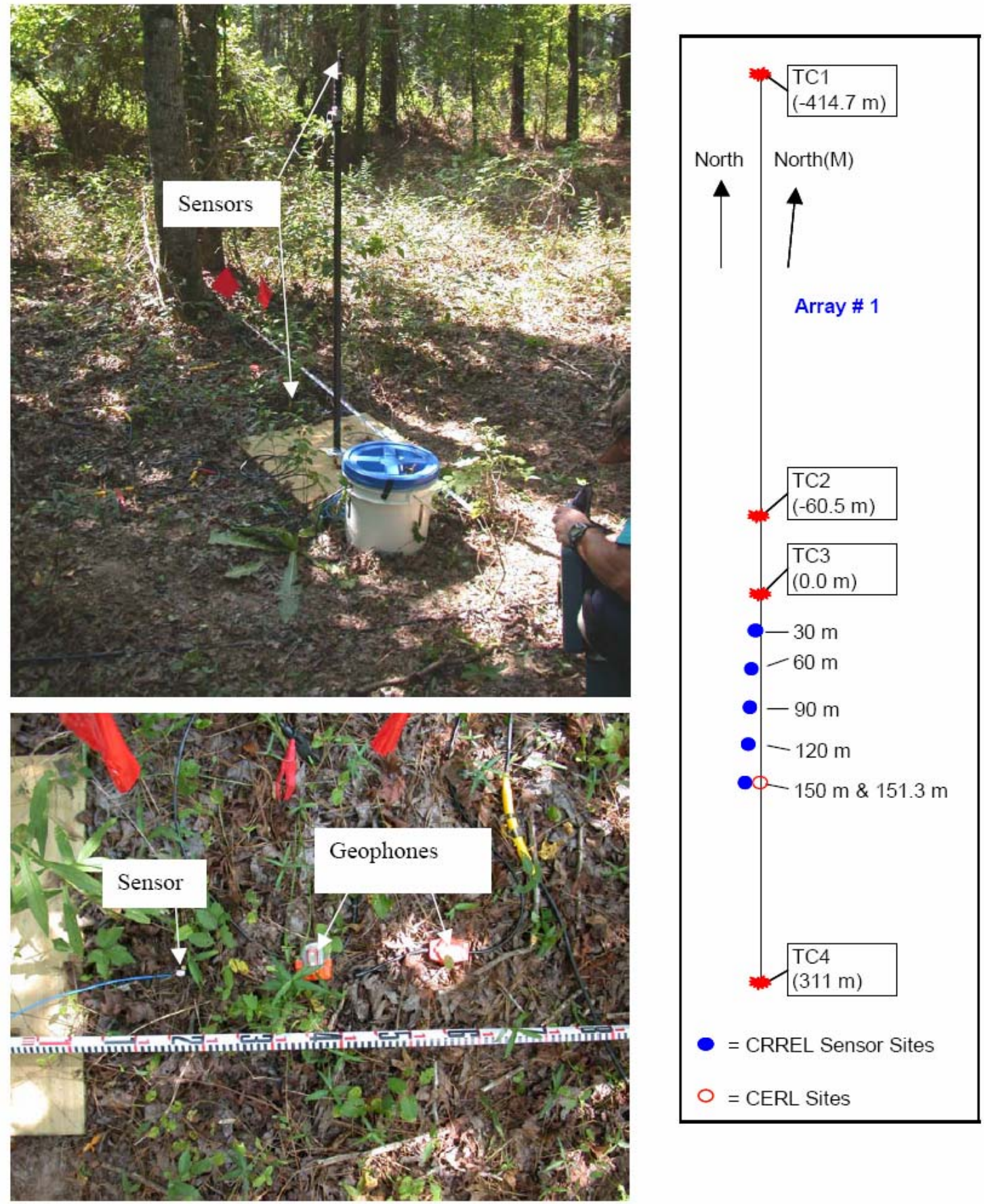

Figure 6. 30 meter sensor locations. 


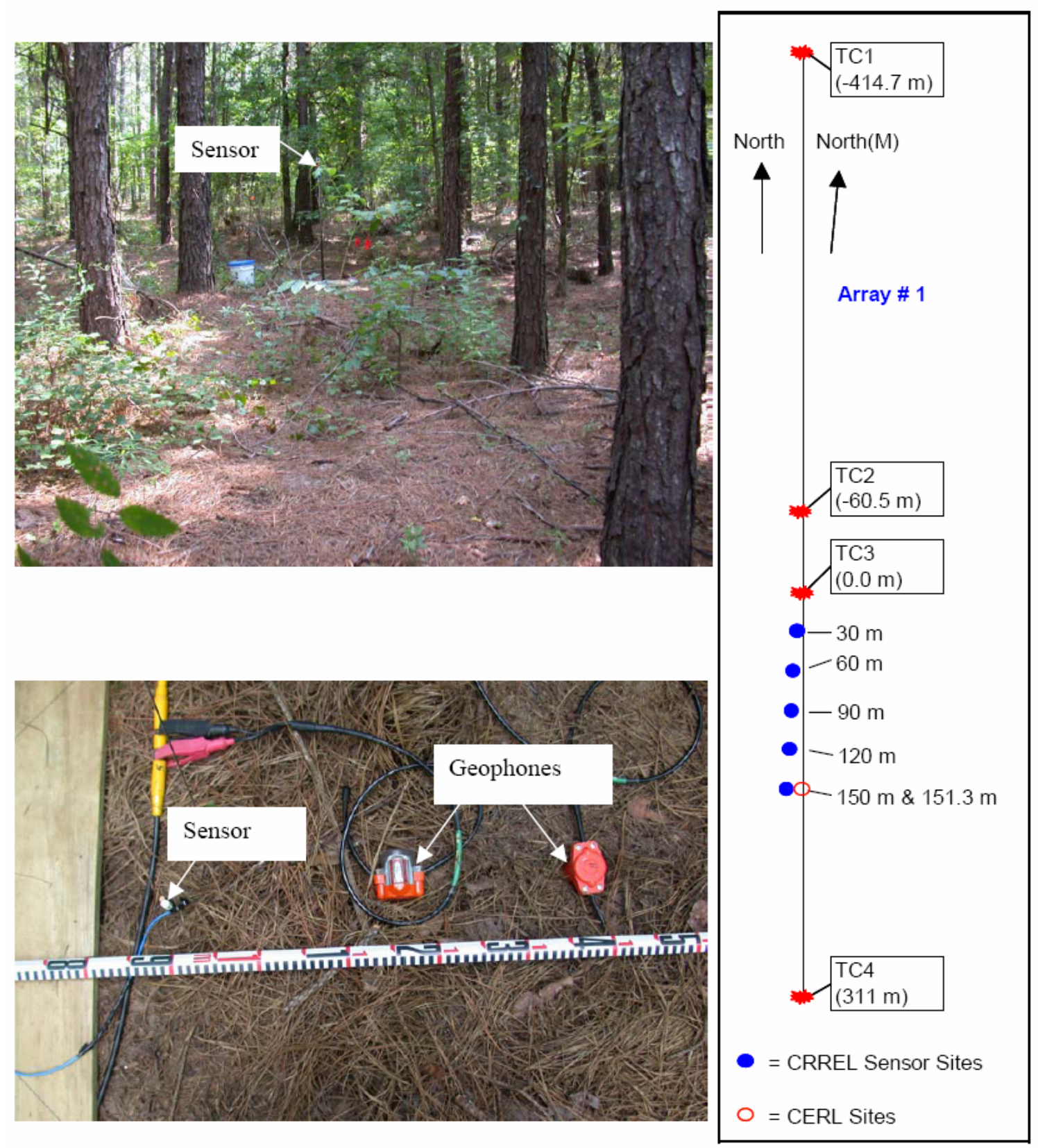

Figure 7. 60 meter sensor locations. 

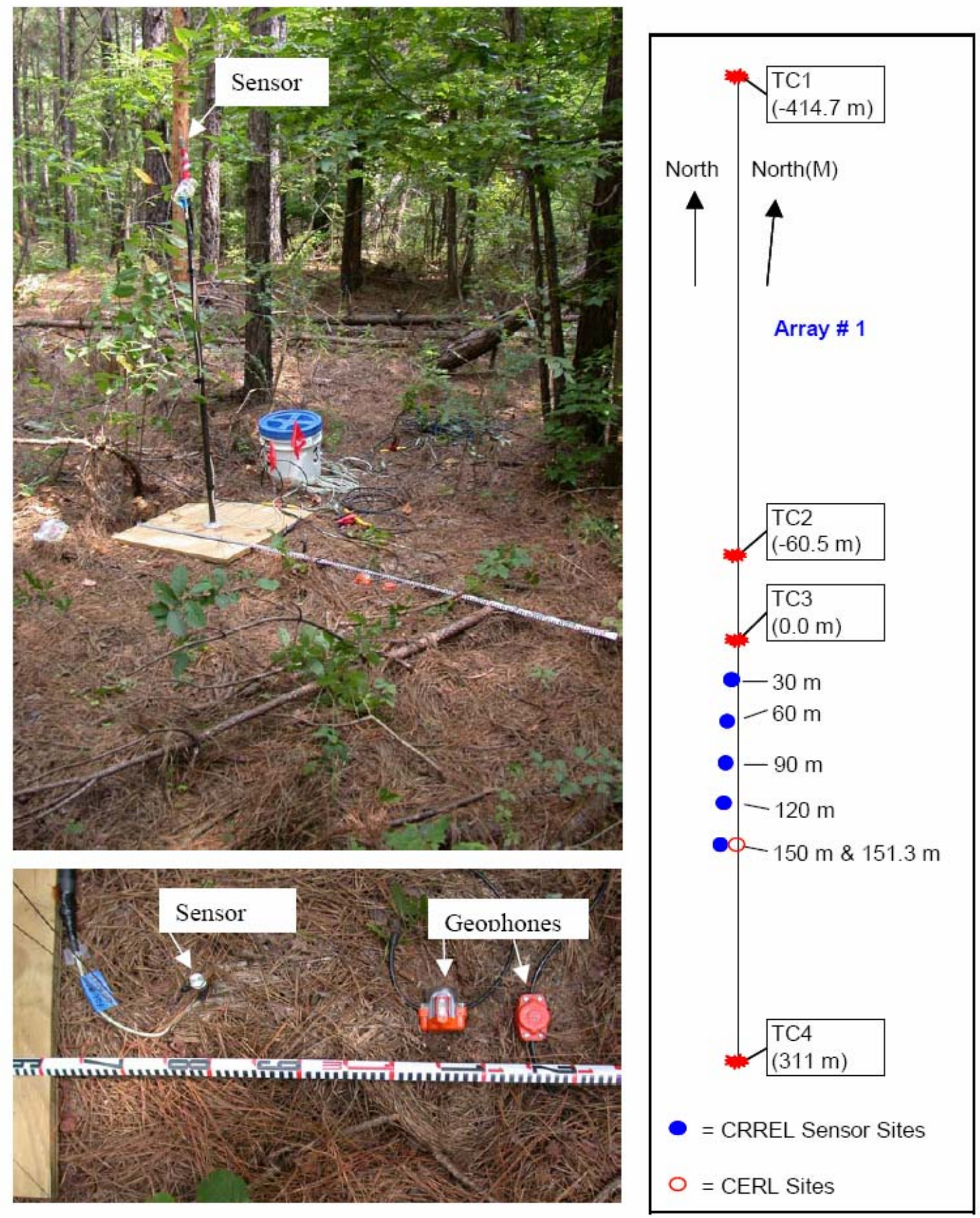

Figure 8. 90 meter sensor locations. 

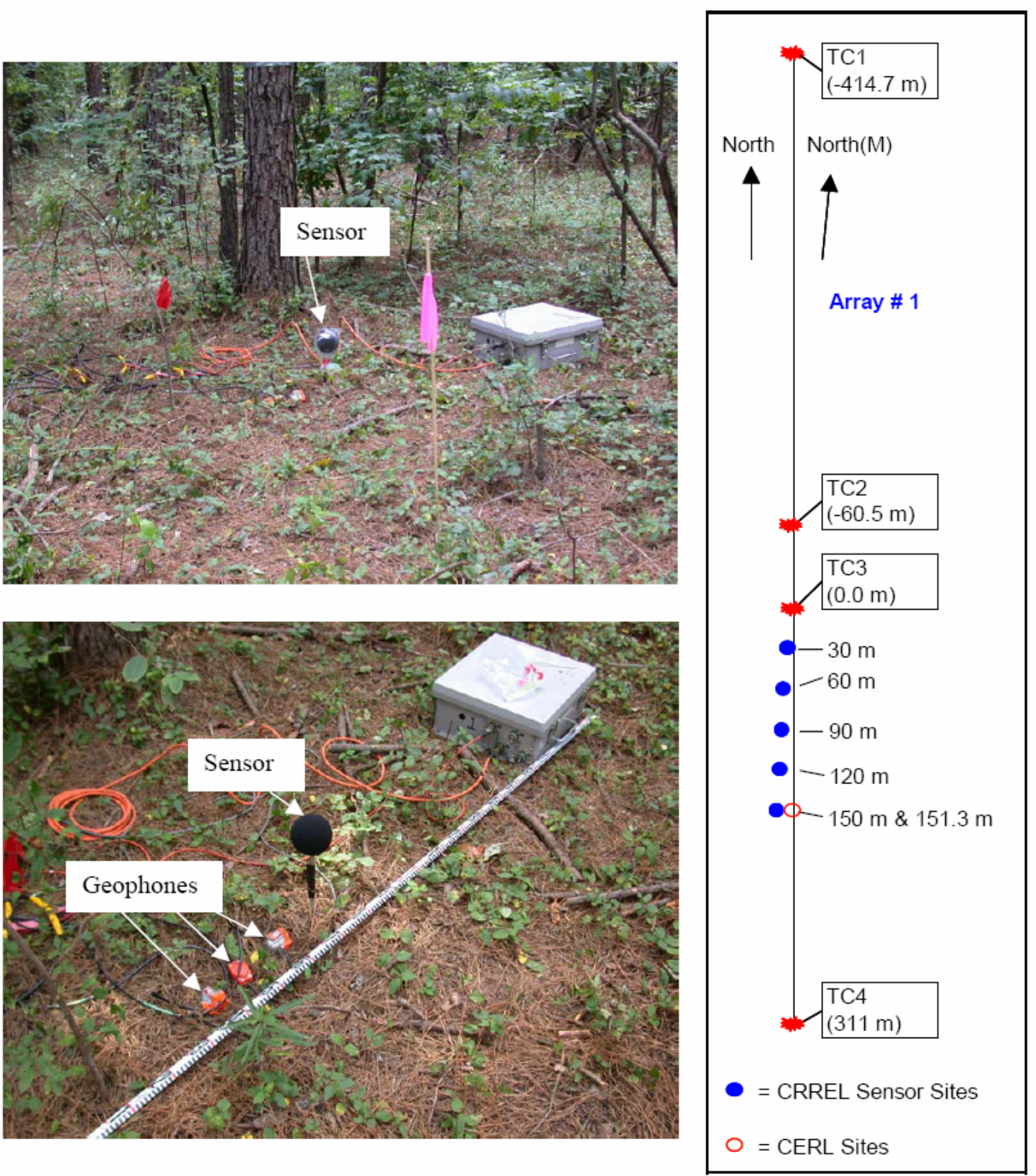

= CERL Sites

Figure 9. 120 meter sensor locations. 

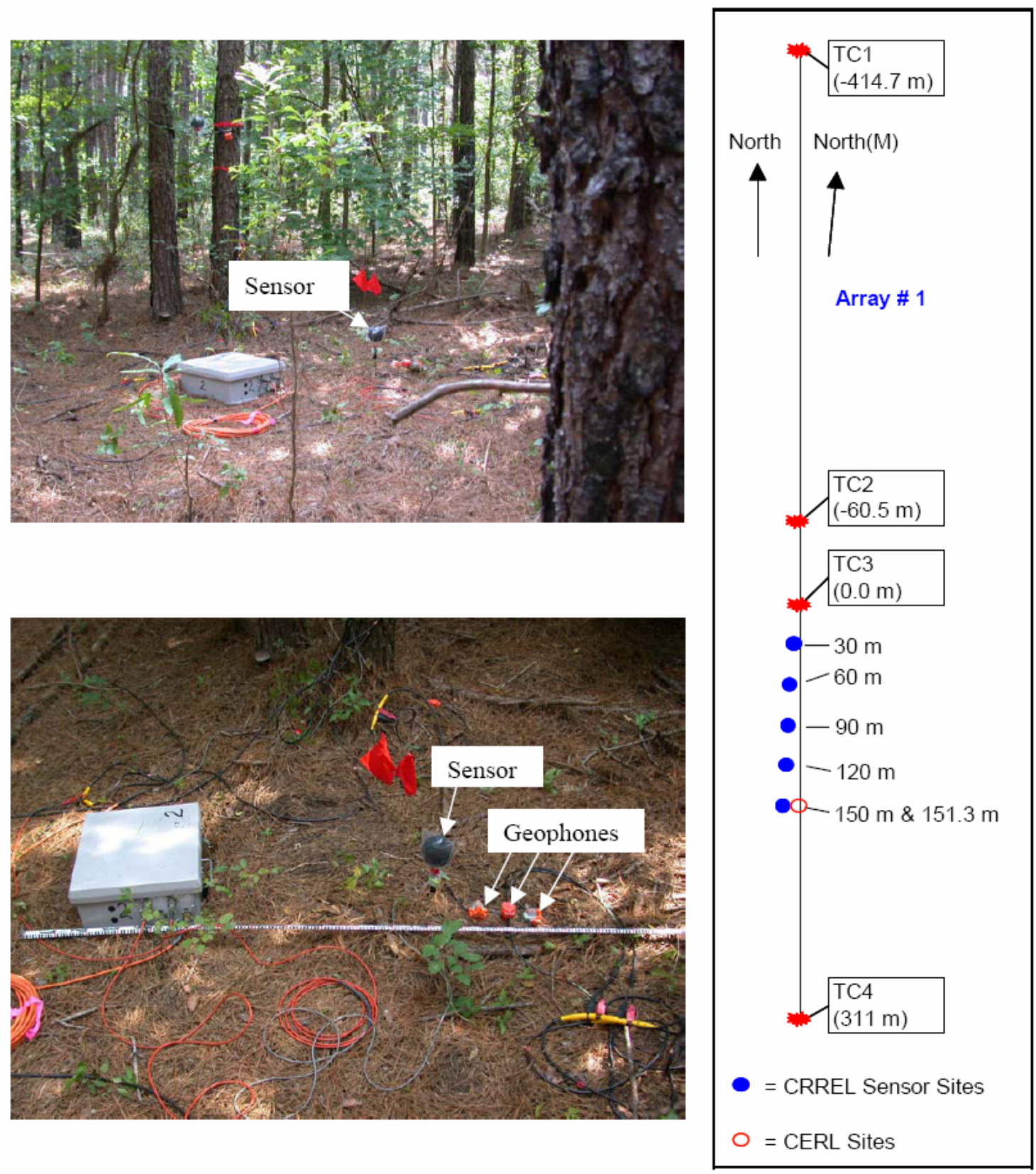

Figure 10. 150 meter sensor locations. 

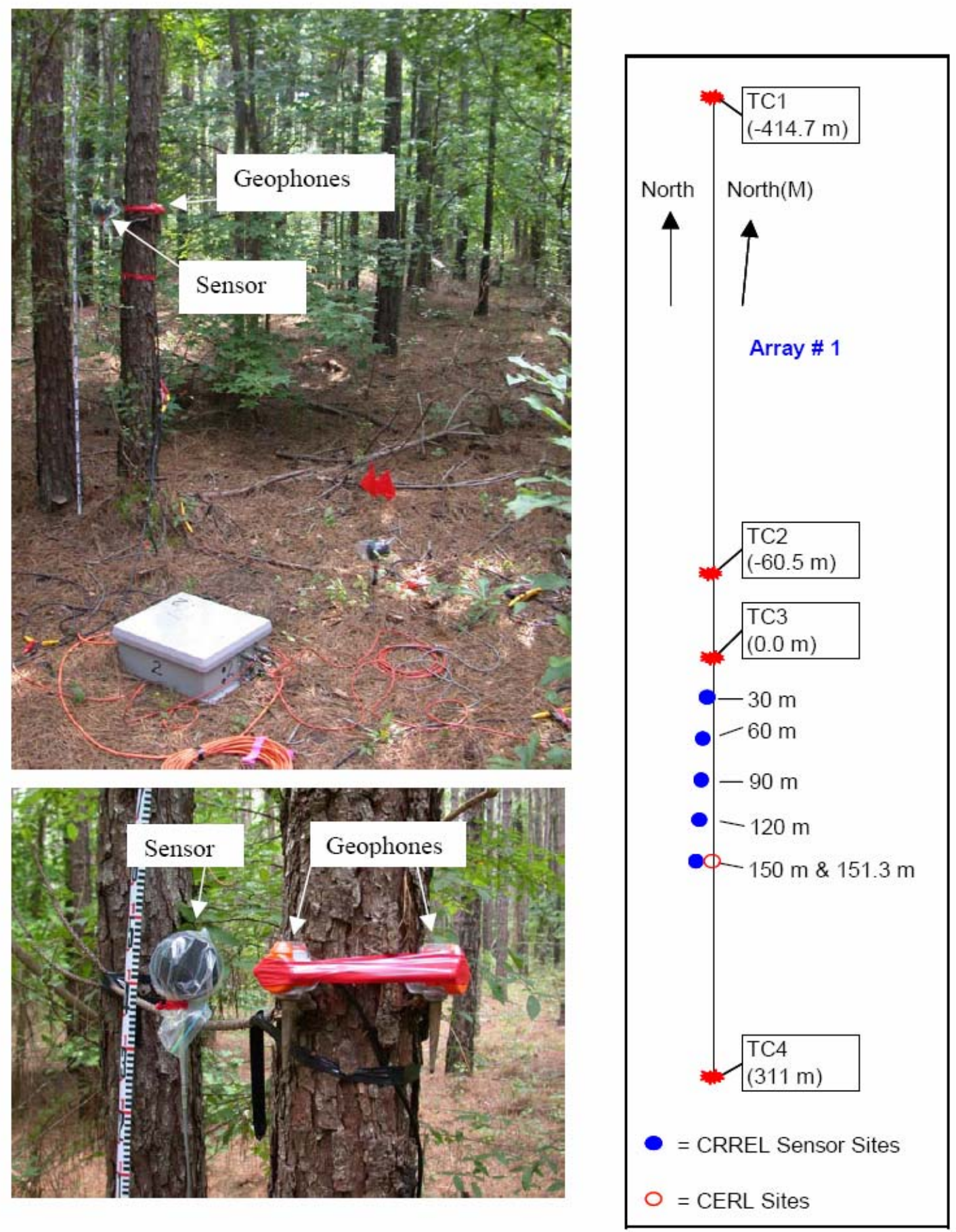

Figure 11. 153.1 meter sensor locations. 


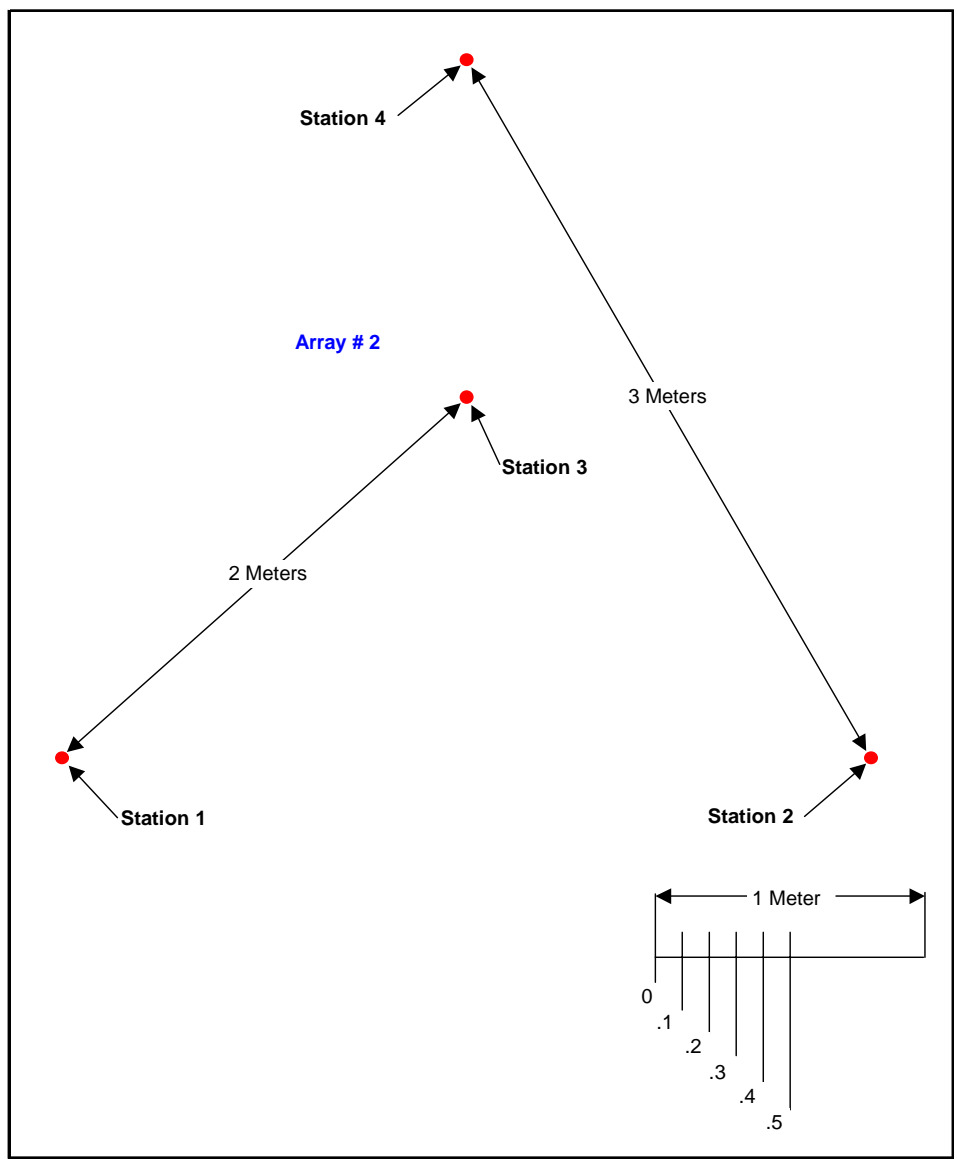

Figure 12. CRREL munitions demolition array. This array was located approximately 600 to $900 \mathrm{~m}$ from the demolition charges, with Station 4 closest to the charges. 
Table 3. Wave propagation sensor array.

Array Cable 1
\begin{tabular}{|c|c|c|c|c|c|}
\hline & $\begin{array}{c}\text { Channel } \\
\text { Location }\end{array}$ & Sumber & $\begin{array}{c}\text { Serial } \\
\text { Number }\end{array}$ & $\begin{array}{c}\text { Height } \\
(\mathbf{m})\end{array}$ & Calibrated Sensitivity \\
\hline 30 Meter & 1 & Radial geophone & $\mathrm{H} 13$ & 0.0 & $32.2 \mathrm{~V} / \mathrm{m} / \mathrm{s}$ \\
\hline 30 Meter & 2 & Vertical geophone & $\mathrm{V} 23$ & 0.0 & $32.2 \mathrm{~V} / \mathrm{m} / \mathrm{s}$ \\
\hline 30 Meter & 3 & PCB 102A07 & 15972 & 0.0 & $1.28 \mathrm{E}-02 \mathrm{mV} / \mathrm{Pa}$ \\
\hline 30 Meter & 4 & PCB 102A07 & 15973 & 1.5 & $1.24 \mathrm{E}-02 \mathrm{mV} / \mathrm{Pa}$ \\
\hline 60 Meter & 5 & Radial geophone & $\mathrm{H} 2$ & 0.0 & $32.2 \mathrm{~V} / \mathrm{m} / \mathrm{s}$ \\
\hline 60 Meter & 6 & Vertical geophone & $\mathrm{V} 11$ & 0.0 & $32.2 \mathrm{~V} / \mathrm{m} / \mathrm{s}$ \\
\hline 60 Meter & 7 & PCB 102A07 & 15971 & 0.0 & $1.30 \mathrm{E}-02 \mathrm{mV} / \mathrm{Pa}$ \\
\hline 60 Meter & 8 & PCB 106B50 & 6693 & 1.5 & $6.74 \mathrm{E}-02 \mathrm{mV} / \mathrm{Pa}$ \\
\hline 90 Meter & 9 & Radial geophone & $\mathrm{H} 18$ & 0.0 & $32.2 \mathrm{~V} / \mathrm{m} / \mathrm{s}$ \\
\hline 90 Meter & 10 & Vertical geophone & V4 & 0.0 & $32.2 \mathrm{~V} / \mathrm{m} / \mathrm{s}$ \\
\hline 90 Meter & 11 & PCB 106B50 & 3259 & 0.0 & $8.17 \mathrm{E}-02 \mathrm{mV} / \mathrm{Pa}$ \\
\hline 90 Meter & 12 & PCB 106B50 & 6695 & 1.5 & $7.16 \mathrm{E}-02 \mathrm{mV} / \mathrm{Pa}$ (uncal) \\
\hline
\end{tabular}

Array Cable 2

\begin{tabular}{|c|c|c|c|c|c|}
\hline & $\begin{array}{c}\text { Channel } \\
\text { Lumber }\end{array}$ & Sensor Number & Sensor S/N & $\begin{array}{c}\text { Height } \\
(\mathbf{m})\end{array}$ & Calibrated Sensitivity \\
\hline 120 Meter & 13 & Radial geophone & $\mathrm{H} 28$ & 0.0 & $32.2 \mathrm{~V} / \mathrm{m} / \mathrm{s}$ \\
\hline 120 Meter & 14 & Transv. geophone & $\mathrm{H} 19$ & 0.0 & $32.2 \mathrm{~V} / \mathrm{m} / \mathrm{s}$ \\
\hline 120 Meter & 15 & Vertical geophone & V6 & 0.0 & $32.2 \mathrm{~V} / \mathrm{m} / \mathrm{s}$ \\
\hline 120 Meter & 16 & B\&K 4938 - 3 & 2239255 & 0.0 & $1.15 \mathrm{E}-3 \mathrm{mV} / \mathrm{Pa}$ \\
\hline 150 Meter & 17 & Radial geophone & $\mathrm{H} 20$ & 0.0 & $32.2 \mathrm{~V} / \mathrm{m} / \mathrm{s}$ \\
\hline 150 Meter & 18 & Transv. geophone & $\mathrm{H} 21$ & 0.0 & $32.2 \mathrm{~V} / \mathrm{m} / \mathrm{s}$ \\
\hline 150 Meter & 19 & Vertical geophone & $\mathrm{V} 28$ & 0.0 & $32.2 \mathrm{~V} / \mathrm{m} / \mathrm{s}$ \\
\hline 150 Meter & 20 & B\&K 4938 - & 2239254 & 0.0 & $1.32 \mathrm{E}-3 \mathrm{mV} / \mathrm{Pa}$ \\
\hline 151.3 Meter & 21 & Radial geophone & $\mathrm{H} 10$ & 1.5 & $32.2 \mathrm{~V} / \mathrm{m} / \mathrm{s}$ \\
\hline 151.3 Meter & 22 & Transv. geophone & $\mathrm{H} 9$ & 1.5 & $32.2 \mathrm{~V} / \mathrm{m} / \mathrm{s}$ \\
\hline 151.3 Meter & 23 & Vertical geophone & $\mathrm{V} ?$ & 1.5 & $32.2 \mathrm{~V} / \mathrm{m} / \mathrm{s}$ \\
\hline 151.3 Meter & 24 & B\&K 4938 - 1 & 2239253 & 1.5 & $1.20 \mathrm{E}-3 \mathrm{mV} / \mathrm{Pa}$ \\
\hline
\end{tabular}

All geophones are Mark Products L-15, $4.5 \mathrm{~Hz}$ All B\&K microphones had 1:1000 attenuation. 
Table 4. Changes to wave propagation sensor array.

The PCB sensor at the $60 \mathrm{~m}$ location failed and was replaced after CRREL binary file number 44 , or CERL shot number 6 .

\begin{tabular}{|c|c|c|c|c|c|}
\hline Location & Channel & Sensor Number & Sensor S/N & Height & Calibrated Sensitivity \\
\hline 60 Meter & 7 & PCB 106B50 & 3260 & 0.0 & $7.16 \mathrm{E}-02 \mathrm{mV} / \mathrm{Pa}$ \\
\hline 60 Meter & 7 & PCB 102A07 & 15971 & 0.0 & $1.30 \mathrm{E}-02 \mathrm{mV} / \mathrm{Pa}$ \\
\hline
\end{tabular}

$A=$ Binary records $45-199$

$\mathrm{B}=$ Binary records $1-44$

The B\&K 4938 microphones at locations $120 \mathrm{~m}$ and $150 \mathrm{~m}$ were replaced with with B\&K 4165 microphones for blank pistol shots 5 - 19, ascii files 105 - 119, plus the associated calibration files.

\begin{tabular}{|c|c|c|c|c|c|}
\hline Location & $\begin{array}{c}\text { Channel } \\
\text { Number }\end{array}$ & Sensor Number & Sensor S/N & $\begin{array}{c}\text { Height } \\
(\mathbf{m})\end{array}$ & Calibrated Sensitivity \\
\hline 120 Meter & $16 \mathrm{~A}$ & B\&K 4938 - 3 & 2239255 & 0.0 & $1.15 \mathrm{E}-3 \mathrm{mV} / \mathrm{Pa}$ \\
\hline 120 Meter & $16 \mathrm{~B}$ & B\&K 4165 -5 & 1881043 & 0.0 & $0.75 \mathrm{E}-3 \mathrm{mV} / \mathrm{Pa}$ \\
\hline 150 Meter & $20 \mathrm{~A}$ & B\&K 4938 - 2 & 2239254 & 0.0 & $1.32 \mathrm{E}-3 \mathrm{mV} / \mathrm{Pa}$ \\
\hline 150 Meter & 20 B & B\&K 4165 - 6 & 1857571 & 0.0 & $0.73 \mathrm{E}-3 \mathrm{mV} / \mathrm{Pa}$ \\
\hline
\end{tabular}

$A=$ Binary records $39-199$

$\mathrm{B}=$ Binary records $5-27$

The 4165 microphones had too low of a calibration value, so may not be reliable. 


\section{SENSOR CALIBRATION}

Tables 3 and 4 list the sensitivities used to convert the voltages recorded by the seismograph to physical units. These two tables also contain the calibration values for all sensors used. The sensitivities were determined as follows:

- Geophones: The sensitivity provided by the manufacturer was used. From past experience at CRREL, these sensors are usually accurate to about $10 \%$, but they can occasionally vary more than this range because of differences in coupling to the ground.

- Pressure Sensors (solid state): The PCB pressure sensors were calibrated in situ using a Trig-Tek Model 402H calibrator (Fig. 13). This model calibrator provides sound pressure levels of 120 to $160 \mathrm{~dB}$ in 10-dB increments. We used levels of 140,150 , and $160 \mathrm{~dB}$ for these calibrations to match the expected blast noise levels. It has selectable internal low frequency outputs of $62.5,125,250,500$ and $1000 \mathrm{~Hz}$. We relied mostly on the lower frequencies, $62.5-250 \mathrm{~Hz}$, for these calibrations.

- Microphones ( $1 / 4$ and $1 / 2$ inch B\&K): The B\&K 1/4 inch Model 4938 microphones were also calibrated using the Trig-Tek Model $402 \mathrm{H}$ calibrator at 140 and $150 \mathrm{~dB}$.

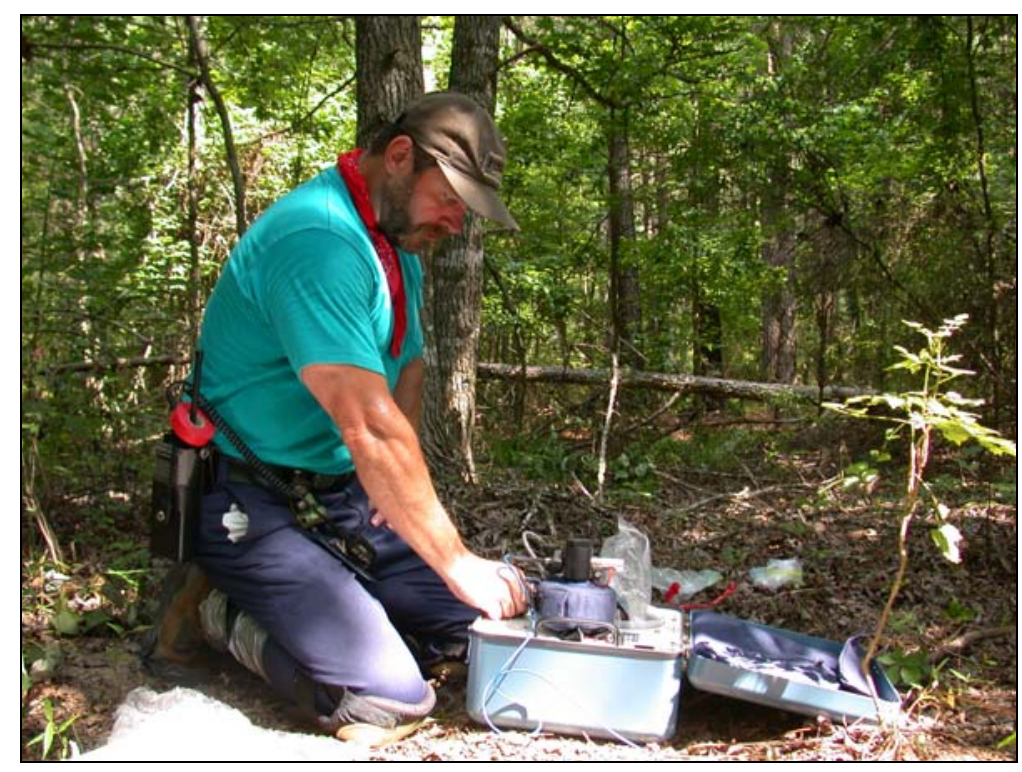

Figure 13. Typical sensor calibration using the Trig-Tek Model $402 \mathrm{H}$ calibrator. 
The B\&K $1 \frac{1}{2}$ inch Model 4165 microphones were calibrated using a GenRad Type 1562-A sound level calibrator. This type of calibrator provides a sound pressure level of $114 \mathrm{~dB}$. It has selectable internal frequency outputs of 125, 250, 500, 1000 and $2000 \mathrm{~Hz}$. 


\section{FOREST ENVIRONMENT}

Figures 13 through 19 show typical views of the conditions within the forest. The understory vegetation limited visibility to about 30-60 m within the forest. Details of the tree species and density are to be provided by forest measurements already conducted as part of the land management at the Lone Star AAP.

Ground conditions usually consisted of a layer of needles a few inches thick, as seen in the photographs. The weather during the tests was very hot and humid, with air temperatures typically near $100^{\circ} \mathrm{F}\left(38^{\circ} \mathrm{C}\right)$.

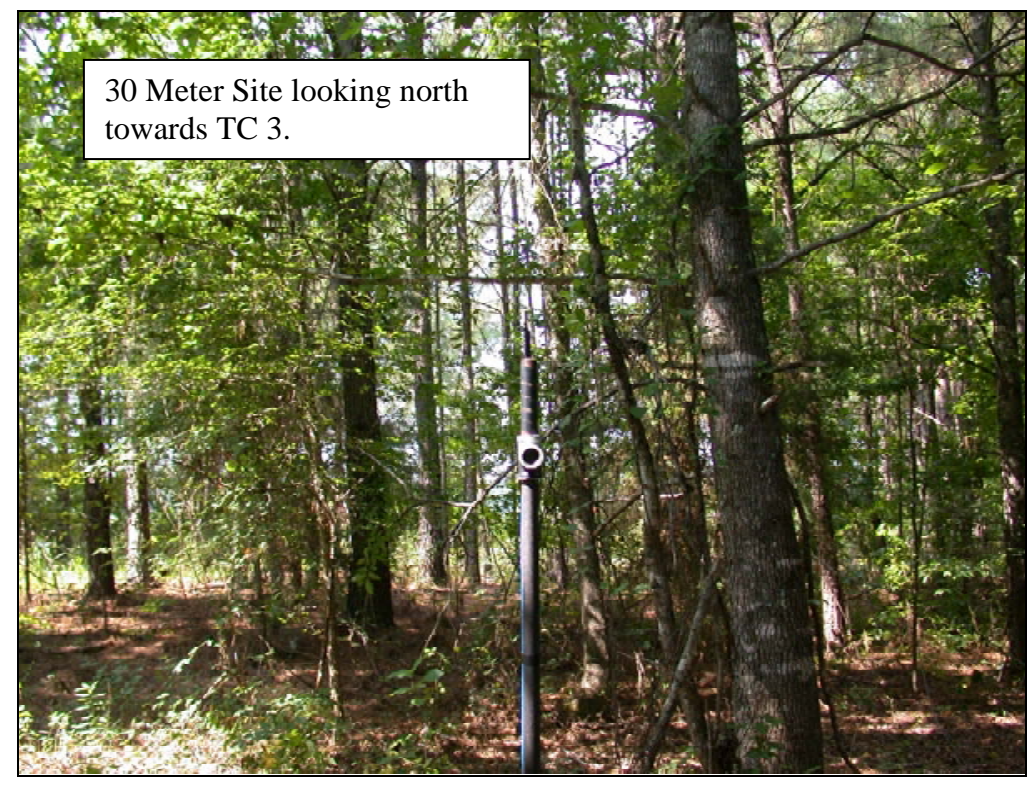

Figure 14. Vegetation at 30 meter site. 


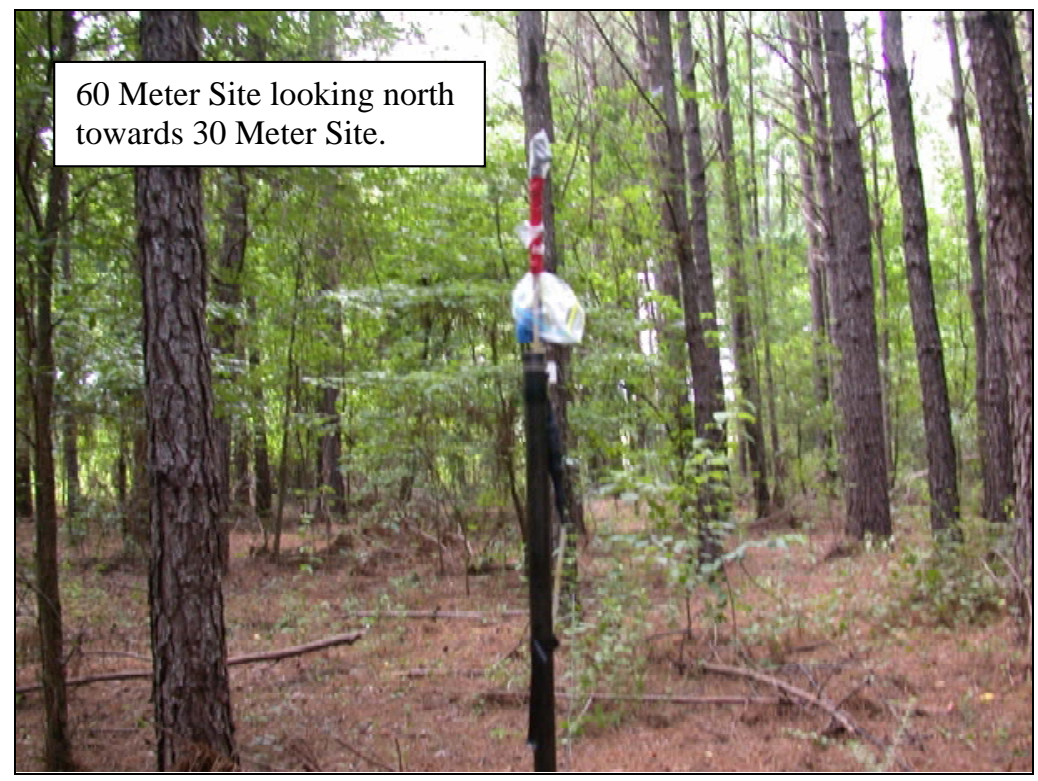

Figure 15. Vegetation at $60 \mathrm{~m}$ site.

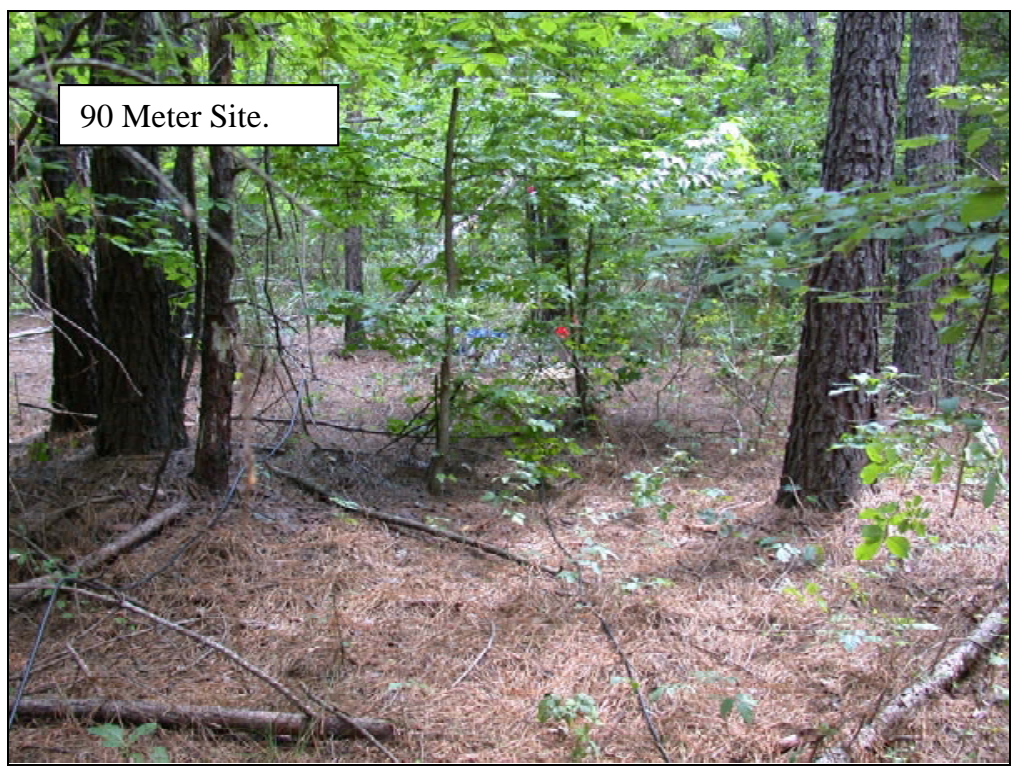

Figure 16. Vegetation at $90 \mathrm{~m}$ site. 


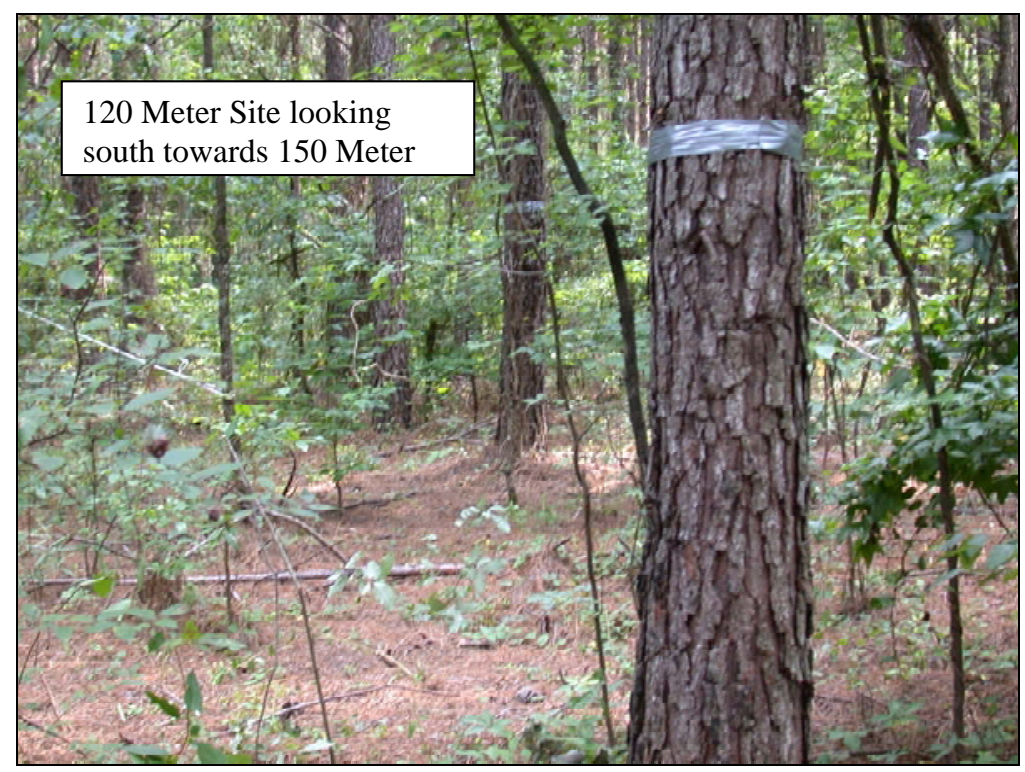

Figure 17. Vegetation at $120 \mathrm{~m}$ site.

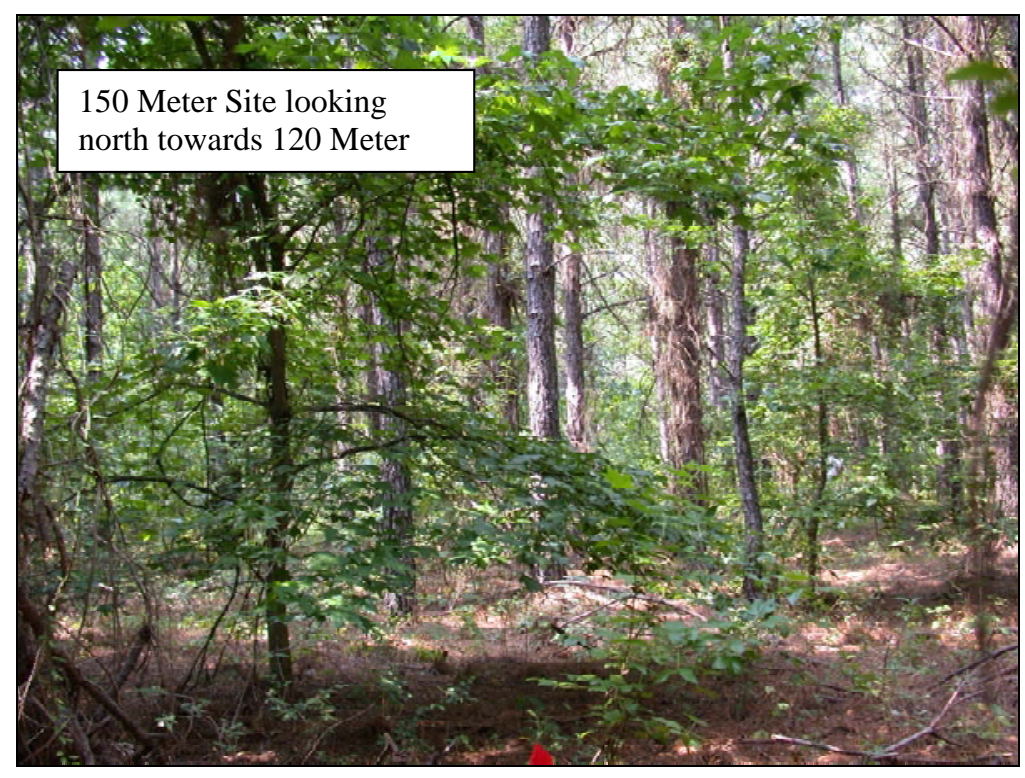

Figure 18. Vegetation at $150 \mathrm{~m}$ site. 


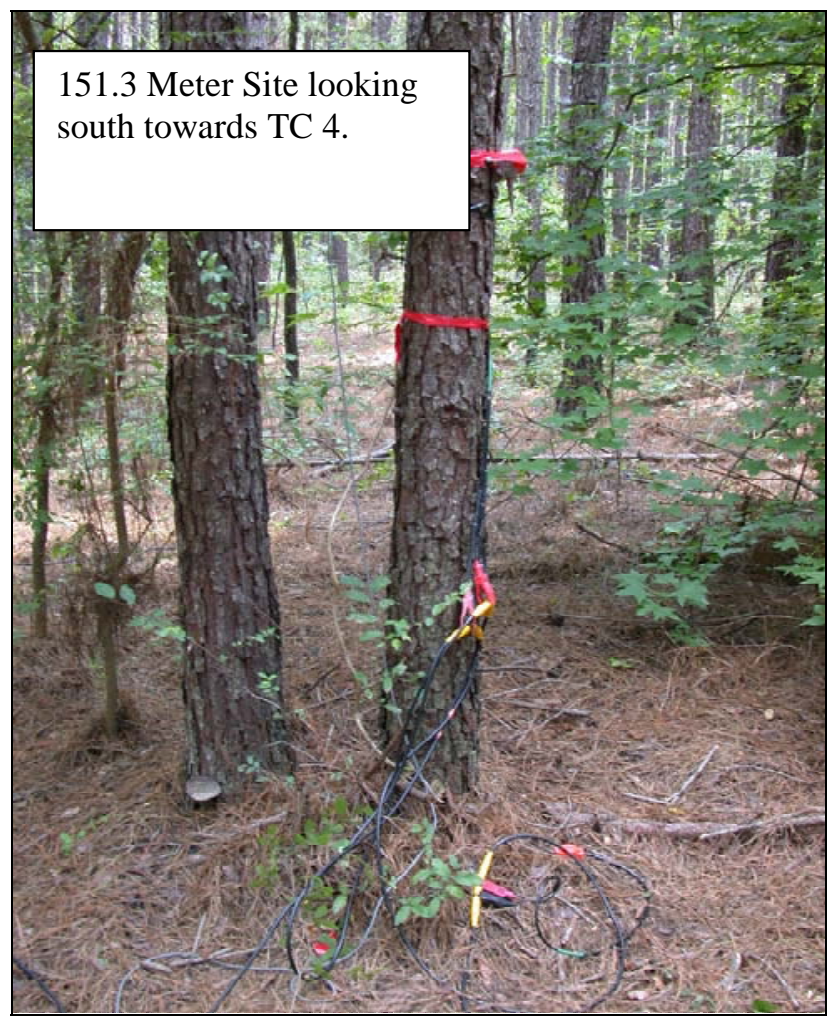

Figure 19. Vegetation at $151.3 \mathrm{~m}$ site. 


\section{TEXAS 2002 TIME BREAK CORRECTIONS AND ACOUSTIC VELOCITIES}

Because of a malfunction with the blaster box, good time breaks were not obtained. The seismograph recordings were started manually during the countdown, so the blast wave arrivals occur at different times within the binary file.

Before writing the ASCII files, the true time break was found by finding the blast wave arrival times and the correct zero time from the acoustic velocity. These corrections are listed below. In the ASCII files, the blast wave arrivals occur at the true arrival time.

In this listing, the shot number is the original CRREL binary file (xx.dat) recorded by the NZ seismograph. For C4 shots, the ASCII file name with the time break corrections applied is tx020xx.asc, where $\mathrm{xx}$ is the CERL number given below. For pistol shots, the

- $\quad$ ASCII file name is tx021xx.asc, where $\mathrm{xx}$ is the CRREL shot number.

- (For shot 40, the binary file is 40.dat and the ascii file is tx2002.asc.)

- (For shot 1, the binary file is 1.dat and the ascii file is tx2101.asc.)

The acoustic velocities listed below for the pistol shots are higher than the true velocities because of the offset of the pistol from the line of the sensors. As the pistol was only $2 \mathrm{~m}$ away from the nearest sensor, this did not affect the time break correction.

The records are sorted by shot location below.

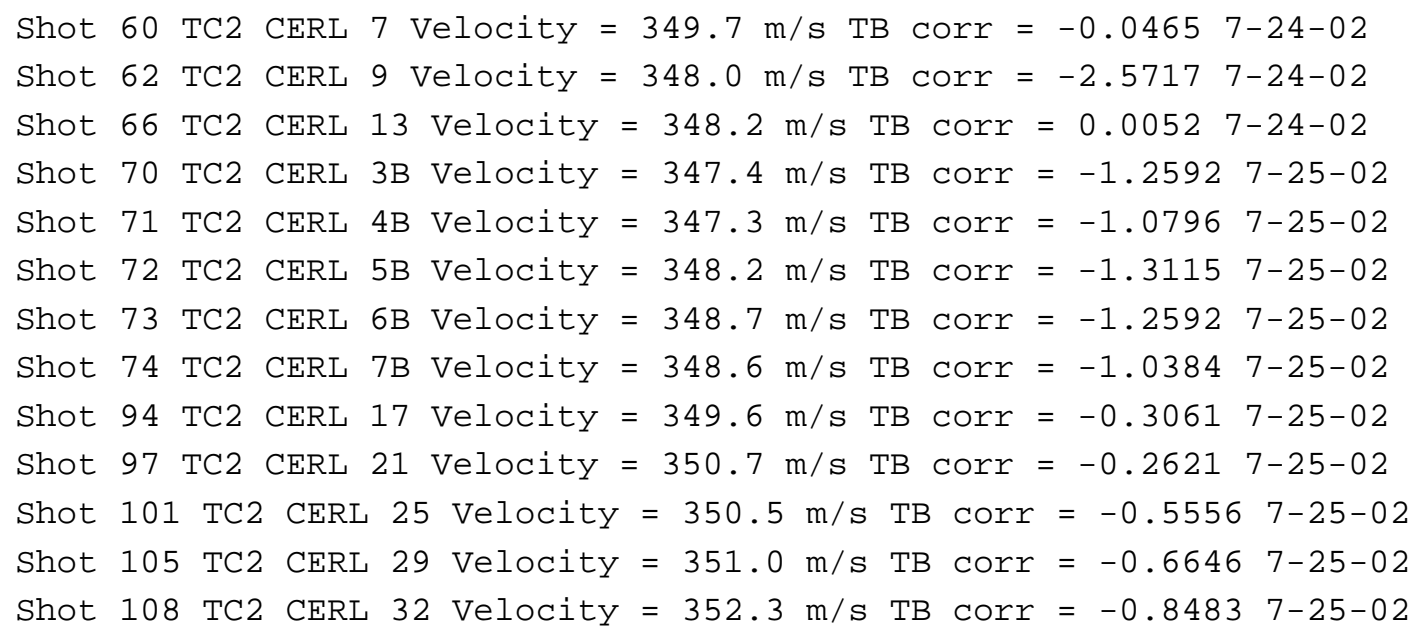


Shot 109 TC2 CERL 33 Velocity $=352.7 \mathrm{~m} / \mathrm{s}$ TB corr $=-1.27427-25-02$

Shot 110 TC2 CERL 34 Velocity $=351.8 \mathrm{~m} / \mathrm{s}$ TB corr $=-1.5879$ 7-25-02

Shot 111 TC2 CERL 35 Velocity $=352.4 \mathrm{~m} / \mathrm{s}$ TB corr $=-1.0272$ 7-25-02

Shot 112 TC2 CERL 36 Velocity $=351.9 \mathrm{~m} / \mathrm{s}$ TB corr $=-0.9628$ 7-25-02

Shot 113 TC2 CERL 37 Velocity $=351.5 \mathrm{~m} / \mathrm{s}$ TB corr $=-1.23907-25-02$

Shot 61 TC1 CERL 8 Velocity $=350.2 \mathrm{~m} / \mathrm{s}$ TB corr $=1.2226$ 7-24-02

Shot 65 TC1 CERL 12 Velocity $=348.6 \mathrm{~m} / \mathrm{s}$ TB corr $=0.26757-24-02$

Shot 93 TC1 CERL 16 Velocity $=352.9 \mathrm{~m} / \mathrm{s}$ TB corr $=-0.42477-25-02$

Shot 96 TC1 CERL 20 Velocity $=348.7 \mathrm{~m} / \mathrm{s}$ TB corr $=-0.27567-25-02$

Shot 100 TC1 CERL 24 Velocity $=351.5 \mathrm{~m} / \mathrm{s}$ TB corr $=-0.69637-25-02$

Shot 104 TC1 CERL 28 Velocity $=351.0 \mathrm{~m} / \mathrm{s}$ TB corr $=-0.81217-25-02$

Shot 40 TC3 CERL 2 Velocity $=350.4 \mathrm{~m} / \mathrm{s}$ TB corr $=-0.21347-24-02$

Shot 41 TC3 CERL 3 Velocity $=349.0 \mathrm{~m} / \mathrm{s}$ TB corr $=-0.21797-24-02$

Shot 42 TC3 CERL 4 Velocity $=350.5 \mathrm{~m} / \mathrm{s}$ TB corr $=-0.21747-24-02$

Shot 67 TC3 CERL 14 Velocity $=348.7 \mathrm{~m} / \mathrm{s}$ TB corr $=0.08807-24-02$

Shot 98 TC3 CERL 22 Velocity $=350.6 \mathrm{~m} / \mathrm{s}$ TB corr $=-1.40867-25-02$

Shot 102 TC3 CERL 26 Velocity $=350.4 \mathrm{~m} / \mathrm{s}$ TB corr $=-0.70767-25-02$

Shot 106 TC3 CERL 30 Velocity $=350.9 \mathrm{~m} / \mathrm{s}$ TB corr $=-0.49057-25-02$

Shot 64 TC4 CERL 11 Velocity $=353.1 \mathrm{~m} / \mathrm{s}$ TB corr $=-0.83797-24-02$

Shot 68 TC4 CERL 15 Velocity $=353.3 \mathrm{~m} / \mathrm{s}$ TB corr $=-1.0028$ 7-24-02

Shot 95 TC4 CERL 19 Velocity $=355.0 \mathrm{~m} / \mathrm{s}$ TB corr $=-1.15807-25-02$

Shot 99 TC4 CERL 23 Velocity $=355.2 \mathrm{~m} / \mathrm{s}$ TB corr $=-1.2413$ 7-25-02

Shot 103 TC4 CERL 27 Velocity $=355.4 \mathrm{~m} / \mathrm{s}$ TB corr $=-1.58337-25-02$

Shot 107 TC4 CERL 31 Velocity $=355.7 \mathrm{~m} / \mathrm{s}$ TB corr $=-2.07207-25-02$

\section{Blank Pistol Shots}

(The velocity is an apparent phase velocity, not the true acoustic velocity, because the source was offset from the sensor line.)

Shot $130 \mathrm{~m}$ Velocity $=362.3 \mathrm{~m} / \mathrm{s}$ TB corr $=0.80917-23-02$

Shot $230 \mathrm{~m}$ Velocity $=362.5 \mathrm{~m} / \mathrm{s}$ TB corr $=0.5928$ 7-23-02

Shot $330 \mathrm{~m}$ Velocity $=362.8 \mathrm{~m} / \mathrm{s}$ TB corr $=0.95997-23-02$

Shot $430 \mathrm{~m}$ Velocity $=363.9 \mathrm{~m} / \mathrm{s}$ TB corr $=1.22687-23-02$

Shot $1530 \mathrm{~m}$ Velocity $=362.3 \mathrm{~m} / \mathrm{s}$ TB corr $=0.93307-24-02$

Shot $1630 \mathrm{~m}$ Velocity $=362.0 \mathrm{~m} / \mathrm{s}$ TB corr $=1.21517-24-02$

Shot $1730 \mathrm{~m}$ Velocity $=362.0 \mathrm{~m} / \mathrm{s}$ TB corr $=1.39547-24-02$

Shot $1830 \mathrm{~m}$ Velocity $=362.0 \mathrm{~m} / \mathrm{s}$ TB corr $=1.1648$ 7-24-02

Shot $1930 \mathrm{~m}$ Velocity $=362.3 \mathrm{~m} / \mathrm{s}$ TB corr $=1.18637-24-02$ 
Shot $590 \mathrm{~m}$ Velocity $=356.9 \mathrm{~m} / \mathrm{s}$ TB corr $=1.40877-24-02$
Shot $690 \mathrm{~m}$ Velocity $=359.0 \mathrm{~m} / \mathrm{s}$ TB corr $=1.47007-24-02$
Shot $790 \mathrm{~m}$ Velocity $=358.2 \mathrm{~m} / \mathrm{s}$ TB corr $=1.07017-24-02$
Shot $890 \mathrm{~m}$ Velocity $=358.5 \mathrm{~m} / \mathrm{s}$ TB corr $=1.08987-24-02$
Shot $990 \mathrm{~m}$ Velocity $=359.0 \mathrm{~m} / \mathrm{s}$ TB corr $=1.23767-24-02$
Shot $1060 \mathrm{~m}$ Velocity $=364.2 \mathrm{~m} / \mathrm{s}$ TB corr $=1.23347-24-02$
Shot $1160 \mathrm{~m}$ Velocity $=364.5 \mathrm{~m} / \mathrm{s}$ TB corr $=1.20907-24-02$
Shot $1260 \mathrm{~m}$ Velocity $=364.5 \mathrm{~m} / \mathrm{s}$ TB corr $=1.28287-24-02$
Shot $1360 \mathrm{~m}$ Velocity $=364.5 \mathrm{~m} / \mathrm{s}$ TB corr $=1.02937-24-02$
Shot $1460 \mathrm{~m}$ Velocity $=364.5 \mathrm{~m} / \mathrm{s}$ TB corr $=1.30297-24-02$ 


\section{SIGNATURE RECORDINGS AND DATA QUALITY}

This section provides plots of all of the C4 test charges and all of the .45 caliber pistol shots recorded by the CRREL sensor array. The plots were produced using the ASCII data files and the MATLAB plot program listed in Appendix C.

Table 5 lists the events recorded during the tests. As explained in Appendix $\mathrm{B}$, the binary data file names were changed to agree with the CERL Test Charge numbering system. Also, to avoid duplicate file names, the .45 caliber shot files were renumbered in the range 101-119.

Table 6 gives an assessment of the data quality of each trace, based on the plots in this section. The table lists "good" data suitable for additional analysis. "Bad" data should not be used, at least not without further processing. The data might be bad because of sensor failure or too high a noise level.

Nine pressure sensor channels were recorded for each of the C4 shots. For the $42 \mathrm{C} 4$ shots detonated during the test, five shots were not recorded because of a failure with the time break signal. Four other channels were unusable, while 15 were usable but noisy.

Appendix A shows each individual record that was made. 
Table 5. Chronology of recorded events.

\begin{tabular}{|c|c|c|c|c|c|c|c|}
\hline $\begin{array}{c}\text { CRREL } \\
\text { File } \\
\text { Name }\end{array}$ & $\begin{array}{c}\text { CERL } \\
\text { Shot } \\
\#\end{array}$ & $\begin{array}{l}\text { ASCII } \\
\text { File } \\
\text { Name }\end{array}$ & Date & $\begin{array}{l}\text { Local } \\
\text { Time }\end{array}$ & Source & $\begin{array}{c}\text { Source Ht. } \\
\mathrm{m} \text { (ft/in.) }\end{array}$ & Comments \\
\hline 1.dat & & TX02101.asc & $07 / 23 / 02$ & 1545 & .45 cal. blank & $1.0(3 / 3)$ & Shot $2 \mathrm{~m}$ east of $30 \mathrm{~m}$ site \\
\hline 2.dat & & TX02102.asc & 07/23/02 & 1553 & .45 cal. blank & $1.0(3 / 3)$ & Shot $2 \mathrm{~m}$ east of $30 \mathrm{~m}$ site \\
\hline 3.dat & & TX02103.asc & $07 / 23 / 02$ & 1555 & .45 cal. blank & $1.0(3 / 3)$ & Shot $2 \mathrm{~m}$ east of $30 \mathrm{~m}$ site \\
\hline 4.dat & & TX02104.asc & 07/23/02 & 1556 & .45 cal. blank & $1.0(3 / 3)$ & Shot $2 \mathrm{~m}$ east of $30 \mathrm{~m}$ site \\
\hline 5.dat & & TX02105.asc & $07 / 24 / 02$ & 0749 & .45 cal. blank & $1.0(3 / 3)$ & Shot $2 \mathrm{~m}$ west of $90 \mathrm{~m}$ site \\
\hline 6.dat & & TX02106.asc & $07 / 24 / 02$ & 0753 & .45 cal. blank & $1.0(3 / 3)$ & Shot $2 \mathrm{~m}$ west of $90 \mathrm{~m}$ site \\
\hline 7.dat & & TX02107.asc & $07 / 24 / 02$ & 0754 & .45 cal. blank & $1.0(3 / 3)$ & Shot $2 \mathrm{~m}$ west of $90 \mathrm{~m}$ site \\
\hline 8.dat & & TX02108.asc & $07 / 24 / 02$ & 0755 & .45 cal. blank & $1.0(3 / 3)$ & Shot $2 \mathrm{~m}$ west of $90 \mathrm{~m}$ site \\
\hline 9.dat & & TX02109.asc & $07 / 24 / 02$ & 0756 & .45 cal. blank & $1.0(3 / 3)$ & Shot $2 \mathrm{~m}$ west of $90 \mathrm{~m}$ site \\
\hline 10.dat & & TX02110.asc & $07 / 24 / 02$ & 0807 & .45 cal. blank & $1.0(3 / 3)$ & Shot $2 \mathrm{~m}$ east of $60 \mathrm{~m}$ site \\
\hline 11.dat & & TX02111.asc & 07/24/02 & 0809 & .45 cal. blank & $1.0(3 / 3)$ & Shot $2 \mathrm{~m}$ east of $60 \mathrm{~m}$ site \\
\hline 12.dat & & TX02112.asc & $07 / 24 / 02$ & 0810 & .45 cal. blank & $1.0(3 / 3)$ & Shot $2 \mathrm{~m}$ east of $60 \mathrm{~m}$ site \\
\hline 13.dat & & TX02113.asc & $07 / 24 / 02$ & 0811 & .45 cal. blank & $1.0(3 / 3)$ & Shot $2 \mathrm{~m}$ east of $60 \mathrm{~m}$ site \\
\hline 14.dat & & TX02114.asc & $07 / 24 / 02$ & 0811 & .45 cal. blank & $1.0(3 / 3)$ & Shot $2 \mathrm{~m}$ east of $60 \mathrm{~m}$ site \\
\hline 15.dat & & TX02115.asc & $07 / 24 / 02$ & 0817 & .45 cal. blank & $1.0(3 / 3)$ & Shot $2 \mathrm{~m}$ east of $30 \mathrm{~m}$ site \\
\hline 16.dat & & TX02116.asc & 07/24/02 & 0818 & .45 cal. blank & $1.0(3 / 3)$ & Shot $2 \mathrm{~m}$ east of $30 \mathrm{~m}$ site \\
\hline 17.dat & & TX02117.asc & $07 / 24 / 02$ & 0818 & .45 cal. blank & $1.0(3 / 3)$ & Shot $2 \mathrm{~m}$ east of $30 \mathrm{~m}$ site \\
\hline 18.dat & & TX02118.asc & 07/24/02 & 0819 & .45 cal. blank & $1.0(3 / 3)$ & Shot $2 \mathrm{~m}$ east of $30 \mathrm{~m}$ site \\
\hline 19.dat & & TX02119.asc & 07/24/02 & 0819 & .45 cal. blank & $1.0(3 / 3)$ & Shot $2 \mathrm{~m}$ east of $30 \mathrm{~m}$ site \\
\hline 20.dat & & & $07 / 24 / 02$ & & Calibration & & 20.dat thru 27. dat are calibration files \\
\hline & & & & & & & \\
\hline 27.dat & & & 07/24/02 & 0909 & Calibration & & \\
\hline 28.dat & & & $07 / 24 / 02$ & 1009 & Munition Demo & & 28.dat thru 38.dat are munition demo files \\
\hline 38.dat & & & $07 / 24 / 02$ & 1014 & Munition Demo & & \\
\hline 39.dat & 1 & & $07 / 24 / 02$ & 1218 & C4 (1 brick) & $1.9(6 / 3)$ & TC2 (See Note 1) \\
\hline 40.dat & 2 & TX02002.asc & $07 / 24 / 02$ & 1235 & C4 (4 bricks) & $1.9(6 / 3)$ & TC2 \\
\hline 41.dat & 3 & TX02003.asc & 07/24/02 & 1334 & C4 (1 brick) & $3.8(12 / 6)$ & TC2 \\
\hline 42.dat & 4 & TX02004.asc & $07 / 24 / 02$ & 1345 & C4 (1 brick) & $3.8(12 / 6)$ & TC2 \\
\hline 43.dat & 5 & & $07 / 24 / 02$ & 1356 & C4 (1 brick) & $1.2(4 / 0)$ & TC2 (See Note 1) \\
\hline 44.dat & 6 & & 07/24/02 & 1406 & C4 (1 brick) & $0.6(2 / 0)$ & TC2 (See Note 1) \\
\hline 45.dat & & & $07 / 24 / 02$ & 1515 & Noise Record & & \\
\hline 46.dat & & & 07/24/02 & 1515 & Munition Demo & & 46. dat thru 56.dat are munition demo files \\
\hline 56.dat & & & 07/24/02 & 1519 & Munition Demo & & \\
\hline 57.dat & & & $07 / 24 / 02$ & 1520 & Noise Record & & \\
\hline 58.dat & & & $07 / 24 / 02$ & 1529 & Noise Record & & \\
\hline 59.dat & & & $07 / 24 / 02$ & 1532 & Munition Demo & & \\
\hline
\end{tabular}

Note 1. CRREL shotbox sent early time break. No ascii file. 
Table 5 (cont'd). Recorded events.

\begin{tabular}{|c|c|c|c|c|c|c|c|c|}
\hline $\begin{array}{c}\text { CRREL } \\
\text { File } \\
\text { Name }\end{array}$ & $\begin{array}{c}\text { CERL } \\
\text { Shot } \\
\#\end{array}$ & $\begin{array}{c}\text { ASCII } \\
\text { File } \\
\text { Name }\end{array}$ & Date & $\begin{array}{l}\text { Local } \\
\text { Time }\end{array}$ & Source & $\begin{array}{c}\text { Source Ht. } \\
\text { m (ft/in.) }\end{array}$ & & Comments \\
\hline 60.dat & 7 & TX02007.asc & $07 / 24 / 02$ & 1550 & C4 (1 brick) & $0.3(1 / 0)$ & & TC2 \\
\hline 61.dat & 8 & TX02008.asc & 07/24/02 & 1613 & C4 (1 brick) & $1.9(6 / 3)$ & & TC1 \\
\hline 62.dat & 9 & TX02009.asc & $07 / 24 / 02$ & 1614 & C4 (1 brick) & $1.9(6 / 3)$ & & TC2 \\
\hline 63.dat & 10 & & $07 / 24 / 02$ & 1615 & C4 (1 brick) & $1.9(6 / 3)$ & & TC3 (See Note 1) \\
\hline 64.dat & 11 & TX02011.asc & 07/24/02 & 1617 & C4 (1 brick) & $1.9(6 / 3)$ & & TC4 \\
\hline 65.dat & 12 & TX02012. asc & $07 / 24 / 02$ & 1631 & C4 (1 brick) & $19(6 / 3)$ & & TC1 \\
\hline 66.dat & 13 & TX02013.asc & $07 / 24 / 02$ & 1633 & C4 (1 brick) & $1.9(6 / 3)$ & & TC2 \\
\hline 67.dat & 14 & TX02014.asc & $07 / 24 / 02$ & 1634 & C4 (1 brick) & $1.9(6 / 3)$ & & TC3 \\
\hline 68.dat & 15 & TX02015.asc & $07 / 24 / 02$ & 1635 & C4 (1 brick) & $1.9(6 / 3)$ & & TC4 \\
\hline 69.dat & & & $07 / 25 / 02$ & 0820 & Noise Record & & & \\
\hline 70.dat & 43 & TX02043.asc & $07 / 25 / 02$ & 0859 & C4 (1 brick) & $3.8(12 / 6)$ & TC2 & Repeat of CERL shot \# 3 \\
\hline 71.dat & 44 & TX02044.asc & $07 / 25 / 02$ & 0910 & C4 (1 brick) & $3.8(12 / 6)$ & TC2 & Repeat of CERL shot \# 4 \\
\hline 72.dat & 45 & TX02045.asc & $07 / 25 / 02$ & 0933 & C4 (1 brick) & $3.0(10 / 0)$ & TC2 & Repeat of CERL shot \# 5 \\
\hline 73.dat & 46 & TX02046.asc & $07 / 25 / 02$ & 0942 & C4 (1 brick) & $1.2(4 / 0)$ & TC2 & Repeat of CERL shot \# 6 \\
\hline 74.dat & 47 & TX02047.asc & $07 / 25 / 02$ & 0951 & C4 (1 brick) & $0.3(1 / 0)$ & TC2 & Repeat of CERL shot \# 7 \\
\hline
\end{tabular}

\begin{tabular}{|c|c|c|c|c|}
\hline 75.dat & 07/25/02 & 1005 & Munition Demo & 75.dat thru 81.dat are munition demo files \\
\hline 81.dat & 07/25/02 & & Munition Demo & \\
\hline 82.dat & $07 / 25 / 02$ & 1013 & Calibration & 82.dat thru 91.dat are munition demo files \\
\hline 91.dat & 07/25/02 & 1019 & Calibration & \\
\hline 92.dat & 07/25/02 & 1103 & Noise Record & \\
\hline
\end{tabular}

\begin{tabular}{|c|c|c|c|c|c|c|c|}
\hline 93.dat & 16 & TX02016.asc & $07 / 25 / 02$ & 1106 & C4 (1 brick) & $1.9(6 / 3)$ & TC1 \\
\hline 94.dat & 17 & TX02017.asc & $07 / 25 / 02$ & 1107 & C4 (1 brick) & $1.9(6 / 3)$ & TC2 \\
\hline & 18 & & $07 / 25 / 02$ & 1107 & C4 (1 brick) & $1.9(6 / 3)$ & TC3 (See note 2) \\
\hline 95.dat & 19 & TX02019.asc & $07 / 25 / 02$ & 1108 & C4 (1 brick) & $1.9(6 / 3)$ & TC4 \\
\hline 96.dat & 20 & TX02020.asc & $07 / 25 / 02$ & 1122 & C4 (5 pounds) & $1.9(6 / 3)$ & TC1 (See note 3) \\
\hline
\end{tabular}

\begin{tabular}{|c|c|c|c|c|c|c|c|}
\hline 97.dat & 21 & TX02021.asc & $07 / 25 / 02$ & 1123 & C4 (5 pounds) & $1.9(6 / 3)$ & TC2 \\
\hline 98.dat & 22 & TX02022.asc & $07 / 25 / 02$ & 1124 & C4 (5 pounds) & $1.9(6 / 3)$ & TC3 \\
\hline 99.9at & 23 & TX02023.asc & $07 / 25 / 02$ & 1125 & C4 (5 pounds) & $1.9(6 / 3)$ & TC4 \\
\hline 100.dat & 24 & TX02024.asc & $07 / 25 / 02$ & 1143 & C4 (5 pounds) & $1.9(6 / 3)$ & TC1 \\
\hline 101.dat & 25 & TX02025.asc & $07 / 25 / 02$ & 1144 & C4 (5 pounds) & $1.9(6 / 3)$ & TC2 \\
\hline
\end{tabular}

\begin{tabular}{|l|c|c|c|c|c|c|c|}
\hline 102.dat & 26 & TX02026.asc & $07 / 25 / 02$ & 1145 & C4 $(5$ pounds $)$ & $1.9(6 / 3)$ & TC3 \\
\hline 103.dat & 27 & TX02027.asc & $07 / 25 / 02$ & 1146 & C4 (5 pounds) & $1.9(6 / 3)$ & TC4 \\
\hline 104.dat & 28 & TX02028.asc & $07 / 25 / 02$ & 1202 & C4 $(5$ pounds $)$ & $1.9(6 / 3)$ & TC1 \\
\hline 105.dat & 29 & TX02029.asc & $07 / 25 / 02$ & 1203 & C4 (5 pounds) & $1.9(6 / 3)$ & TC2 \\
\hline 106.dat & 30 & TX02030.asc & $07 / 25 / 02$ & 1204 & C4 $(5$ pounds $)$ & $1.9(6 / 3)$ & TC3 \\
\hline
\end{tabular}

Note 2. Time break failed. No binary or ascii file.

Note 3. All C-4 charges detonated to create files 96.dat through 113.dat were done using spherical charges, not preformed bricks. 
Table 5 (cont'd).

\begin{tabular}{|c|c|c|c|c|c|c|c|}
\hline $\begin{array}{c}\text { CRREL } \\
\text { File } \\
\text { Name }\end{array}$ & $\begin{array}{c}\text { CERL } \\
\text { Shot } \\
\#\end{array}$ & $\begin{array}{c}\text { ASCII } \\
\text { File } \\
\text { Name }\end{array}$ & Date & $\begin{array}{l}\text { Local } \\
\text { Time }\end{array}$ & Source & $\begin{array}{c}\text { Source Ht. } \\
\text { m (ft/in.) }\end{array}$ & Comments \\
\hline 107.dat & 31 & TX02031.asc & $07 / 25 / 02$ & 1204 & C4 (5 pounds) & $1.9(6 / 3)$ & TC4 \\
\hline 108.dat & 32 & TX02032.asc & 07/25/02 & 1316 & C4 (5 pounds) & $1.9(6 / 3)$ & TC2 \\
\hline 109.dat & 33 & TX02033.asc & $07 / 25 / 02$ & 1325 & C4 (5 pounds) & $3.8(12 / 6)$ & $\mathrm{TC} 2$ \\
\hline 110.dat & 34 & TX02034.asc & $07 / 25 / 02$ & 1336 & C4 (5 pounds) & $3.8(12 / 6)$ & TC2 \\
\hline 111.dat & 35 & TX02035.asc & $07 / 25 / 02$ & 1347 & C4 (5 pounds) & $3.0(10 / 0)$ & TC2 \\
\hline
\end{tabular}

\begin{tabular}{|l|c|c|c|c|c|c|c|}
\hline 112.dat & 36 & TX02036.asc & $07 / 25 / 02$ & 1356 & C4 (5 pounds) & $1.2(4 / 0)$ & TC2 \\
\hline 113.dat & 37 & TX02037.asc & $07 / 25 / 02$ & 1406 & C4 (5 pounds) & $0.3(1 / 0)$ & TC2 \\
\hline 114.dat & & & $07 / 25 / 02$ & 1543 & Calibration & & 114.dat thru 199.dat are calibration files \\
\hline 199.dat & & & $07 / 25 / 02$ & 1648 & Calibration & & \\
\hline
\end{tabular}

Table 6. Data quality.

TX 2002 Forest Test

Sensors - Channel numbers

\section{C4 Shots}

\begin{tabular}{|c|c|c|c|c|c|c|c|c|c|c|c|c|c|c|c|c|c|c|c|c|c|c|c|}
\hline CERL & & & & & & & & & & & & & & & & & & & & & & & \\
\hline Rec Num -> & 1 & 2 & 3 & 4 & 5 & 6 & 7 & 8 & 9 & 10 & 11 & 12 & 13 & 14 & 15 & 16 & 17 & 18 & 19 & 20 & 21 & 22 & 23 \\
\hline CRREL & & & & & & & & & & & & & & & & & & & & & & & \\
\hline Rec Num-> & 39 & 40 & 41 & 42 & 43 & 44 & 60 & 61 & 62 & 63 & 64 & 65 & 66 & 67 & 68 & 93 & 94 & $x$ & 95 & 96 & 97 & 98 & 99 \\
\hline 4 P-30-1 & $x$ & $\mathrm{O}$ & $\mathrm{O}$ & $\mathrm{O}$ & $x$ & $x$ & $\mathrm{O}$ & $\mathrm{O}$ & $\mathrm{O}$ & $x$ & $\mathrm{O}$ & $\mathrm{O}$ & $\mathrm{O}$ & $\mathrm{O}$ & $\mathrm{O}$ & $\mathrm{O}$ & $\mathrm{O}$ & $x$ & $\mathrm{O}$ & $\mathrm{O}$ & $\mathrm{O}$ & $\mathrm{O}$ & $\mathrm{O}$ \\
\hline 3 P-30-0 & $x$ & $\mathrm{O}$ & $\mathrm{O}$ & $\mathrm{O}$ & $x$ & $x$ & $\mathrm{O}$ & $N$ & $\mathrm{O}$ & $x$ & $\mathrm{~N}$ & $N$ & $\mathrm{O}$ & $\mathrm{O}$ & $\mathrm{O}$ & $\mathrm{O}$ & $\mathrm{O}$ & $x$ & $\mathrm{O}$ & $x$ & $\mathrm{O}$ & $\mathrm{O}$ & $\mathrm{O}$ \\
\hline $8 \mathrm{P}-60-1$ & $x$ & $\mathrm{O}$ & $\mathrm{O}$ & O & $x$ & $x$ & $\mathrm{O}$ & $\mathrm{O}$ & $\mathrm{O}$ & $x$ & $\mathrm{O}$ & $\mathrm{O}$ & $\mathrm{O}$ & $\mathrm{O}$ & $\mathrm{O}$ & $\mathrm{O}$ & $\mathrm{O}$ & $x$ & $\mathrm{O}$ & $\mathrm{O}$ & $\mathrm{O}$ & $\mathrm{O}$ & $\mathrm{O}$ \\
\hline 7 P-60-0 & $x$ & $x$ & $x$ & $x$ & $x$ & $x$ & $\mathrm{O}$ & 0 & $\mathrm{O}$ & $x$ & $\mathrm{O}$ & $\mathrm{O}$ & $\mathrm{O}$ & $\mathrm{O}$ & O & $\mathrm{O}$ & $\mathrm{O}$ & $x$ & $\mathrm{O}$ & $\mathrm{O}$ & $\mathrm{O}$ & $\mathrm{O}$ & $\mathrm{O}$ \\
\hline 12 P-90-1 & $x$ & $\mathrm{O}$ & $\mathrm{O}$ & $\mathrm{O}$ & $x$ & $x$ & $\mathrm{O}$ & $\mathrm{O}$ & $\mathrm{O}$ & $x$ & $\mathrm{O}$ & $\mathrm{O}$ & $\mathrm{O}$ & $\mathrm{O}$ & $\mathrm{O}$ & $\mathrm{O}$ & $\mathrm{O}$ & $x$ & $\mathrm{O}$ & $\mathrm{O}$ & $\mathrm{O}$ & $\mathrm{O}$ & $\mathrm{O}$ \\
\hline 11 P-90-0 & $x$ & $\mathrm{O}$ & $\mathrm{O}$ & $\mathrm{O}$ & $x$ & $x$ & $\mathrm{O}$ & $\mathrm{O}$ & $\mathrm{O}$ & $x$ & $\mathrm{O}$ & $\mathrm{O}$ & $\mathrm{O}$ & $\mathrm{O}$ & $\mathrm{O}$ & $\mathrm{O}$ & $\mathrm{O}$ & $x$ & $\mathrm{O}$ & $\mathrm{O}$ & $\mathrm{O}$ & $\mathrm{O}$ & $\mathrm{O}$ \\
\hline 16 P-120-0 & $x$ & $\mathrm{O}$ & O & O & $x$ & $x$ & $\mathrm{O}$ & $N$ & $\mathrm{O}$ & $x$ & $\mathrm{O}$ & $N$ & $\mathrm{O}$ & $\mathrm{O}$ & $\mathrm{O}$ & $\mathrm{O}$ & $\mathrm{O}$ & $x$ & $\mathrm{O}$ & $N$ & $\mathrm{O}$ & $\mathrm{O}$ & $\mathrm{O}$ \\
\hline 20 P-150-0 & $x$ & $\mathrm{O}$ & O & $\mathrm{O}$ & $x$ & $x$ & $\mathrm{O}$ & $\mathrm{N}$ & $\mathrm{O}$ & $x$ & $\mathrm{O}$ & $\mathrm{N}$ & $\mathrm{O}$ & $\mathrm{O}$ & $\mathrm{O}$ & $\mathrm{O}$ & $\mathrm{O}$ & $x$ & $\mathrm{O}$ & $\mathrm{N}$ & $\mathrm{O}$ & $\mathrm{O}$ & $\mathrm{O}$ \\
\hline 4 P-152T-1 & $x$ & $\mathrm{O}$ & $\mathrm{O}$ & $\mathrm{O}$ & $x$ & $x$ & $\mathrm{O}$ & $\mathrm{N}$ & $\mathrm{O}$ & $x$ & $\mathrm{O}$ & $\mathrm{N}$ & $\mathrm{O}$ & $\mathrm{O}$ & $\mathrm{O}$ & $\mathrm{O}$ & $\mathrm{O}$ & $x$ & $\mathrm{O}$ & $\mathrm{N}$ & $\mathrm{O}$ & $\mathrm{O}$ & $\mathrm{O}$ \\
\hline
\end{tabular}

Shot box TB failed for CERL shots 1, 5, 6, 10. No ascii files.

TB failure for CERL shot 18 , no binary or ascii file. 


\section{Table 6 (cont'd).}

\section{C4 Shots}

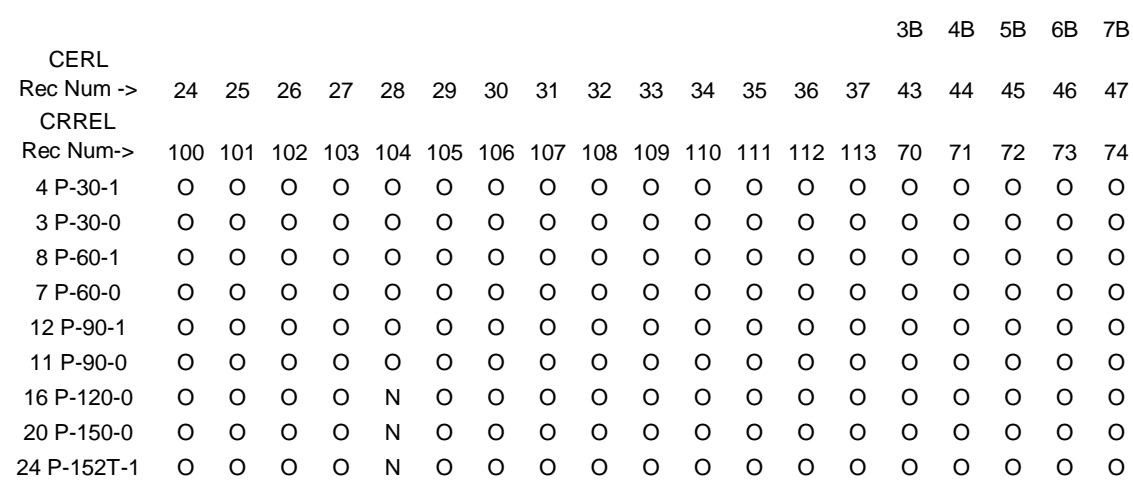

$\mathrm{O}=\mathrm{Good}$

$\mathrm{X}=\mathrm{Bad}$

$\mathrm{N}=$ Noisy data - but usable

$\mathrm{R}=$ Ringing sensor

$\mathrm{C}=$ Crosstalk

\section{BLANK PISTOL SHOTS}

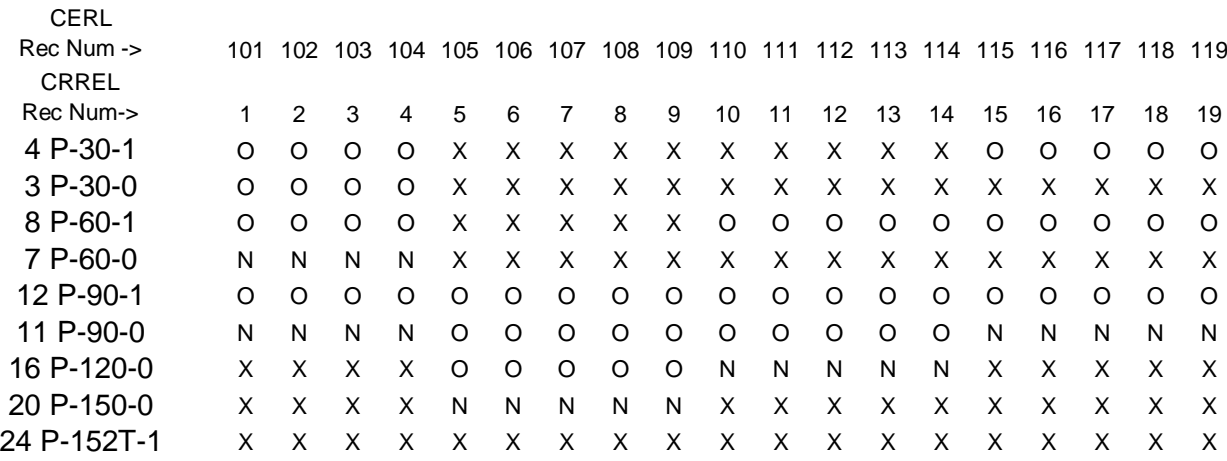

For records 105-119, channels 16, 20 used B\&K 4165 microphones.

$$
\begin{aligned}
& O=\text { Good } \\
& X=\text { Bad } \\
& N=\text { Noisy data }- \text { but usable } \\
& R=\text { Ringing sensor } \\
& C=\text { Crosstalk }
\end{aligned}
$$




\section{APPENDIX A: SIGNAL DATA PLOTS}

The following signal data plots show the response of the 24 sensors recorded during each test. The plots are normalized so that each channel is the same size. The maximum amplitude is shown in the label for each channel. Figure A1 describes the plot layout, labels and descriptions used for each record made during the test. The plots are normalized so that each channel is the same size. The maximum amplitude is shown in the label for each channel.

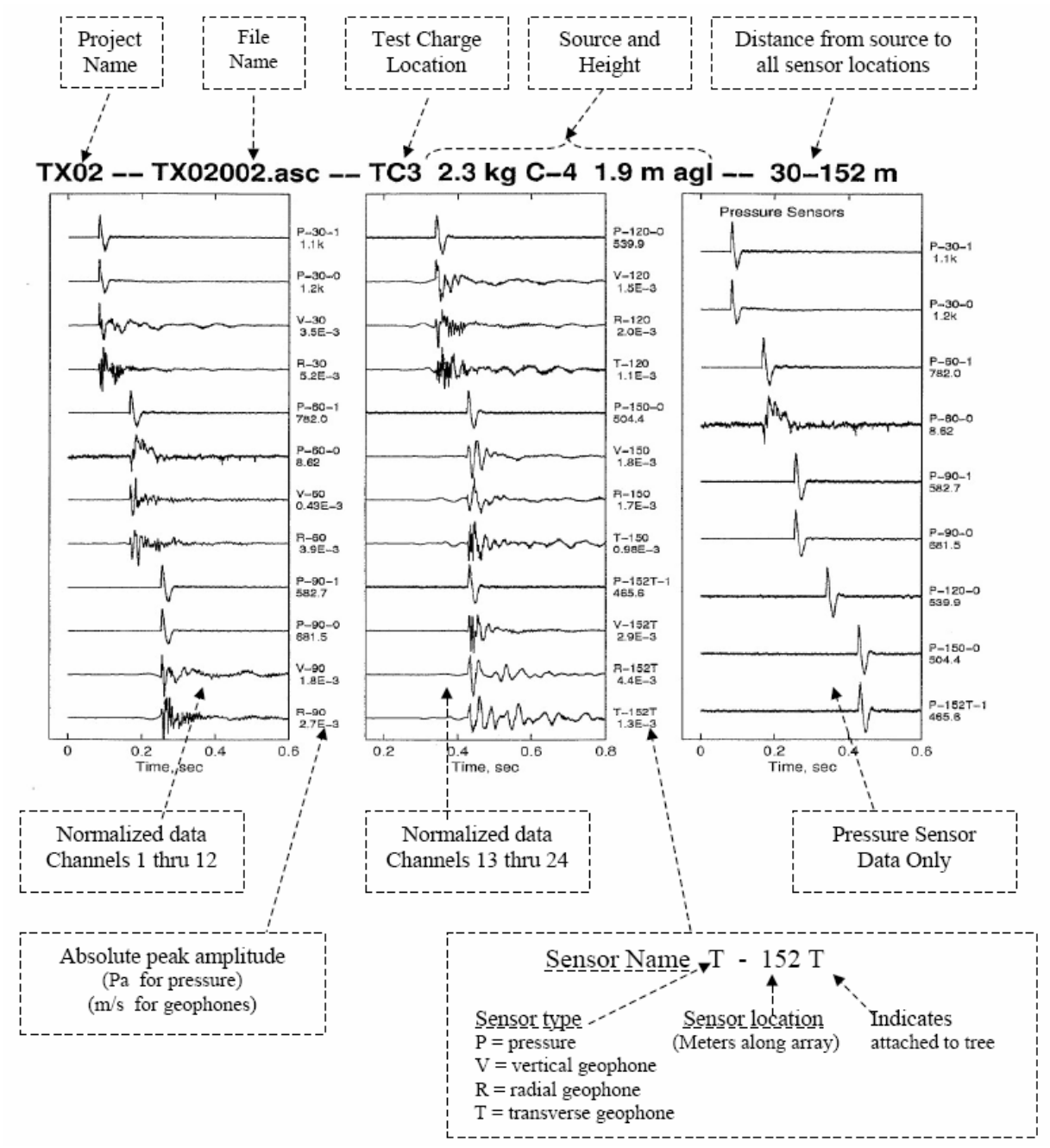

Figure A1. Description of the signal data plots. 


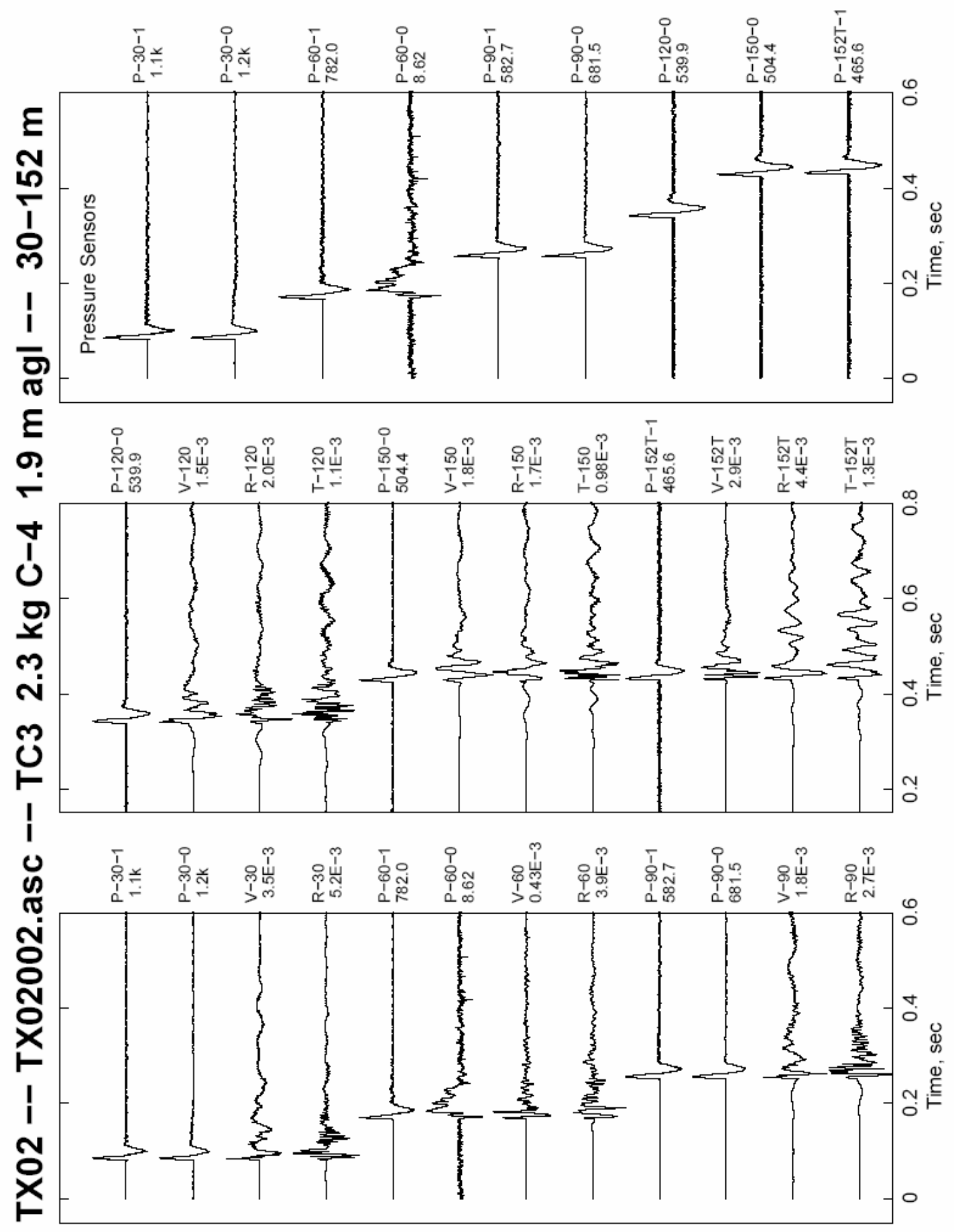




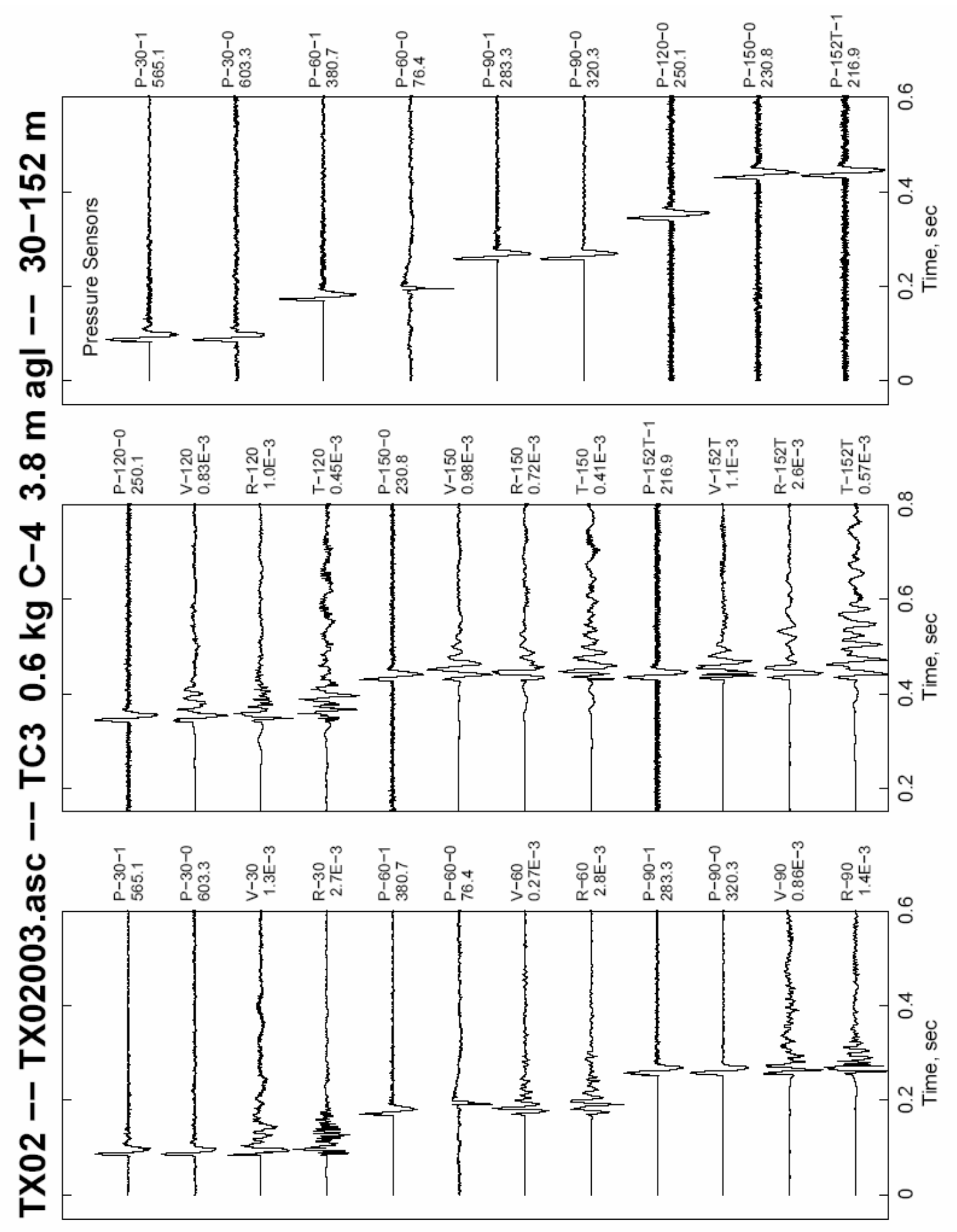




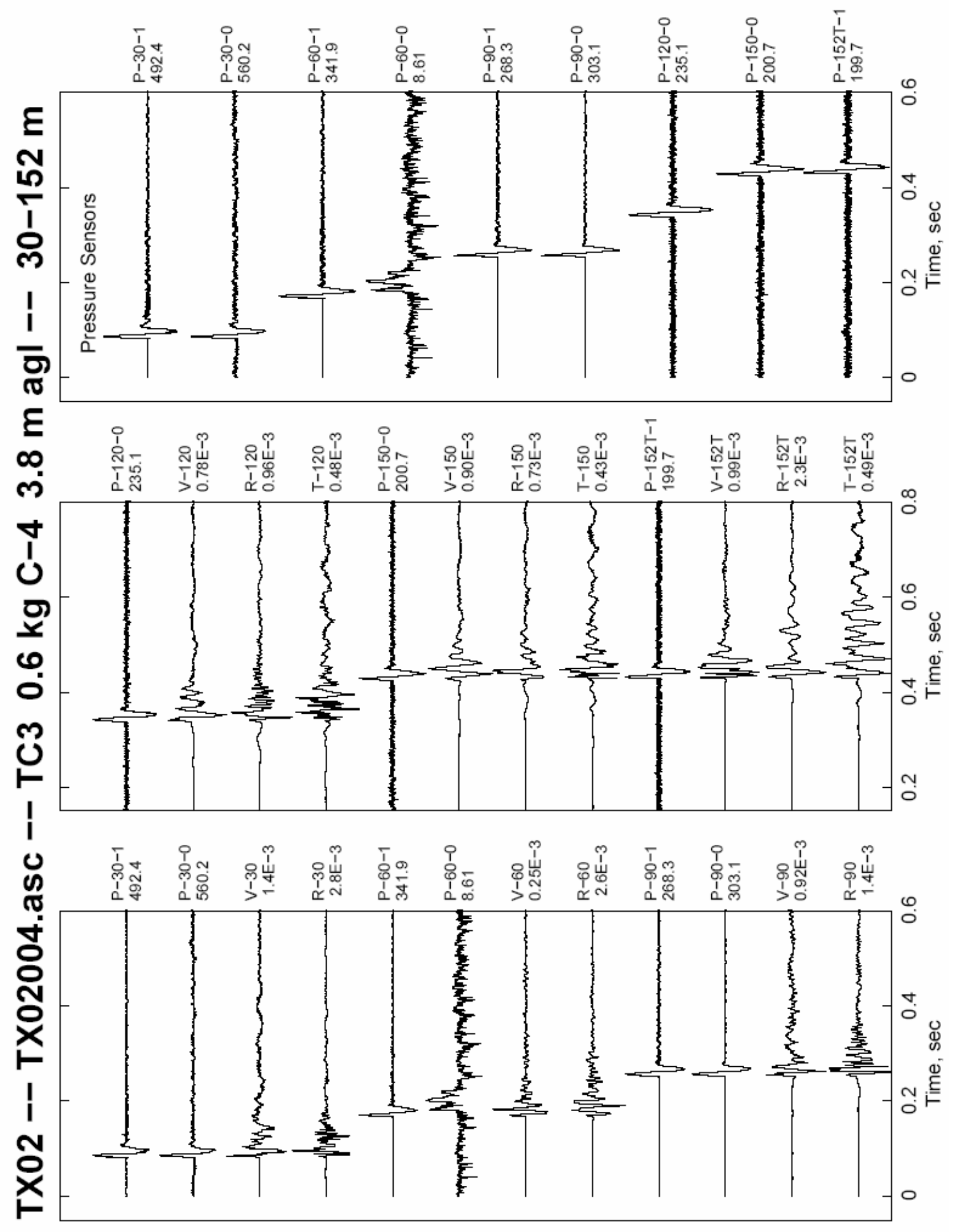




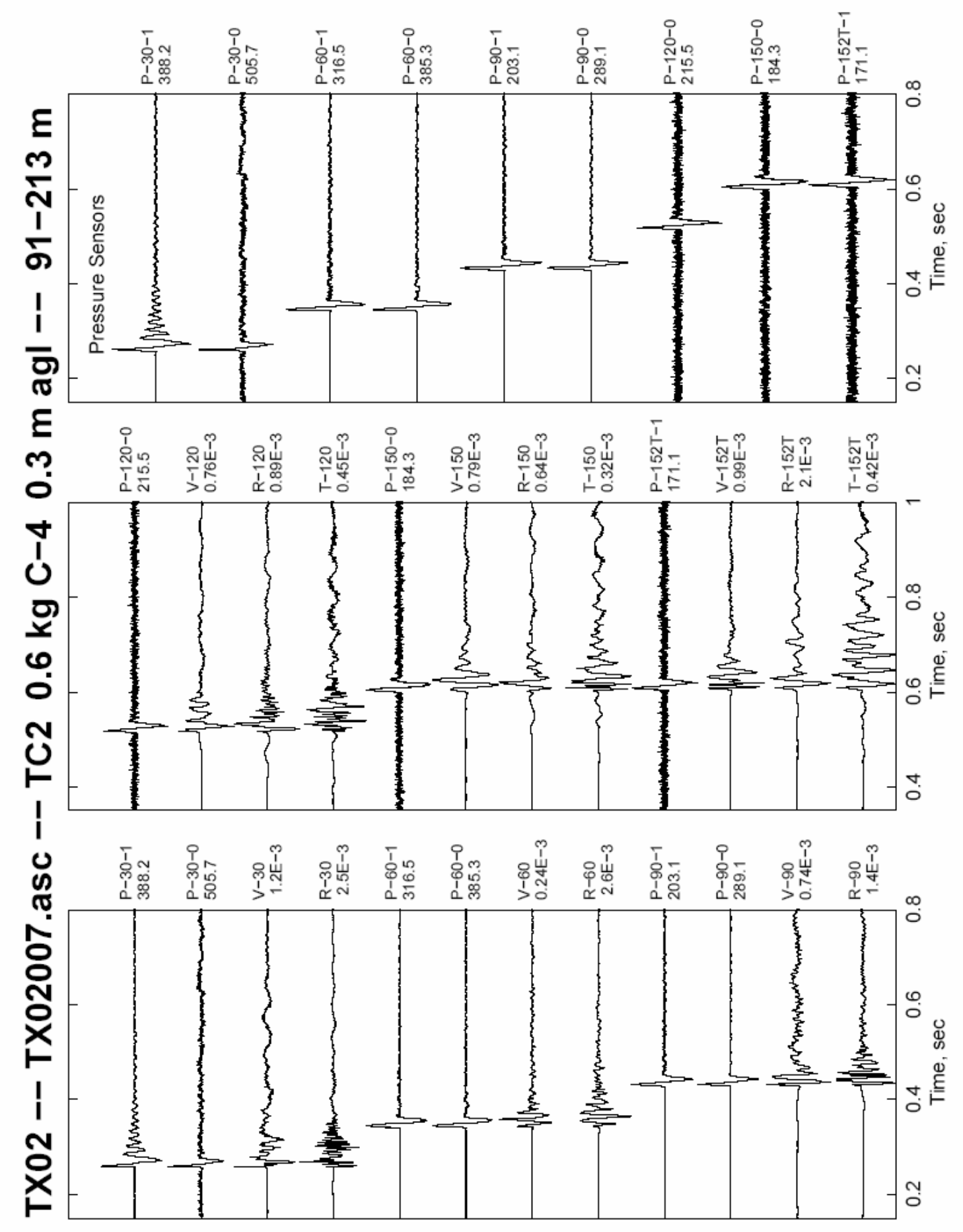




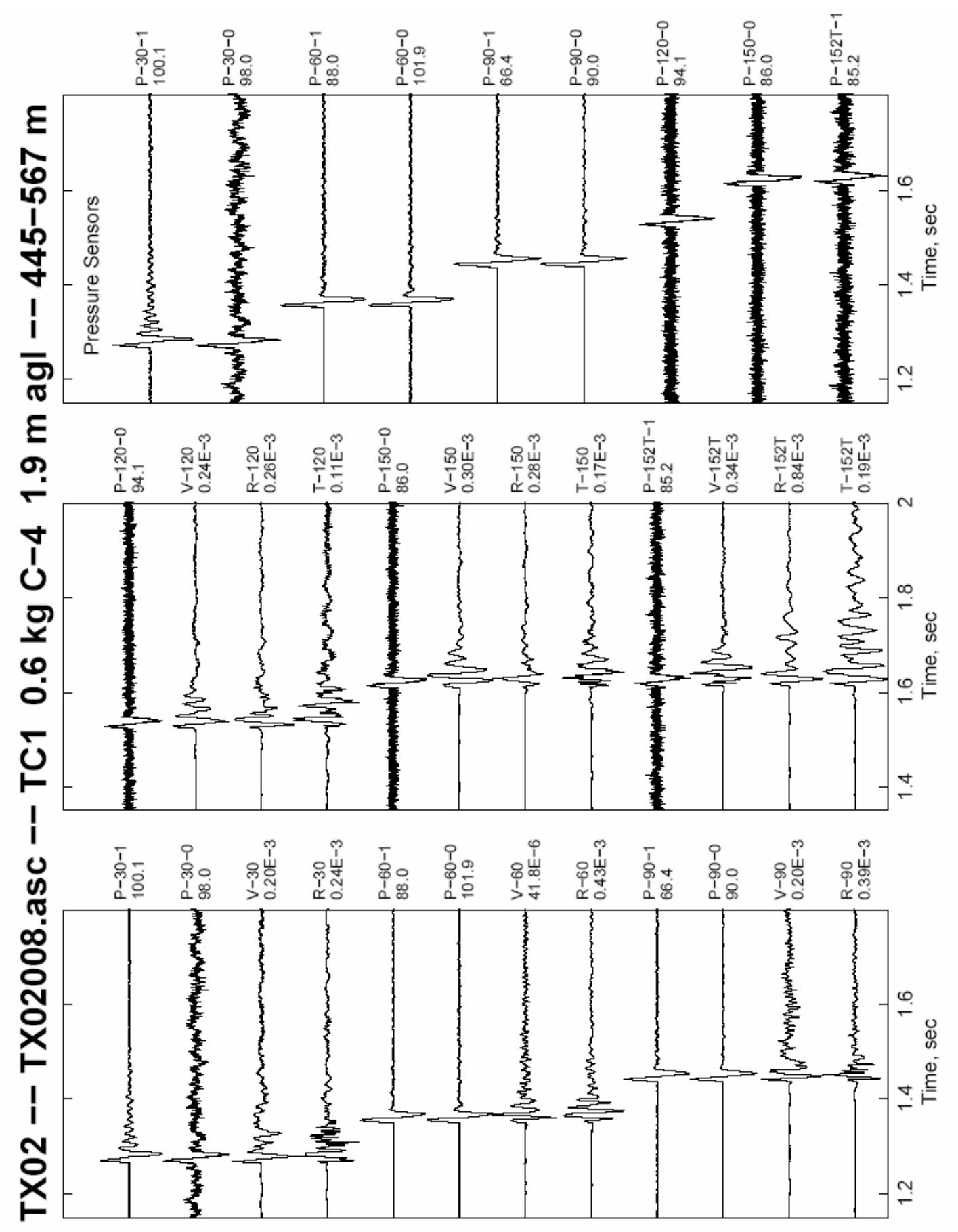




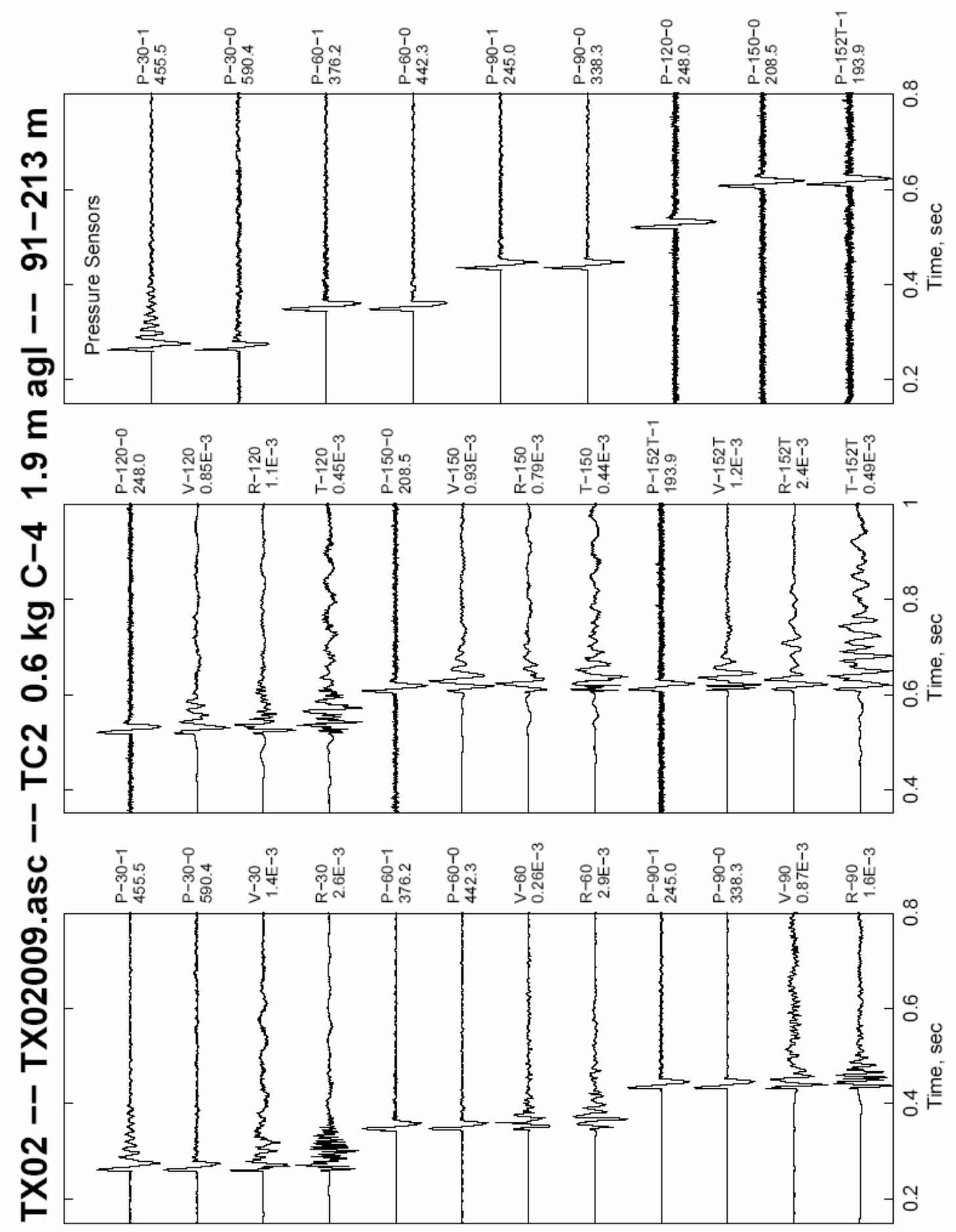




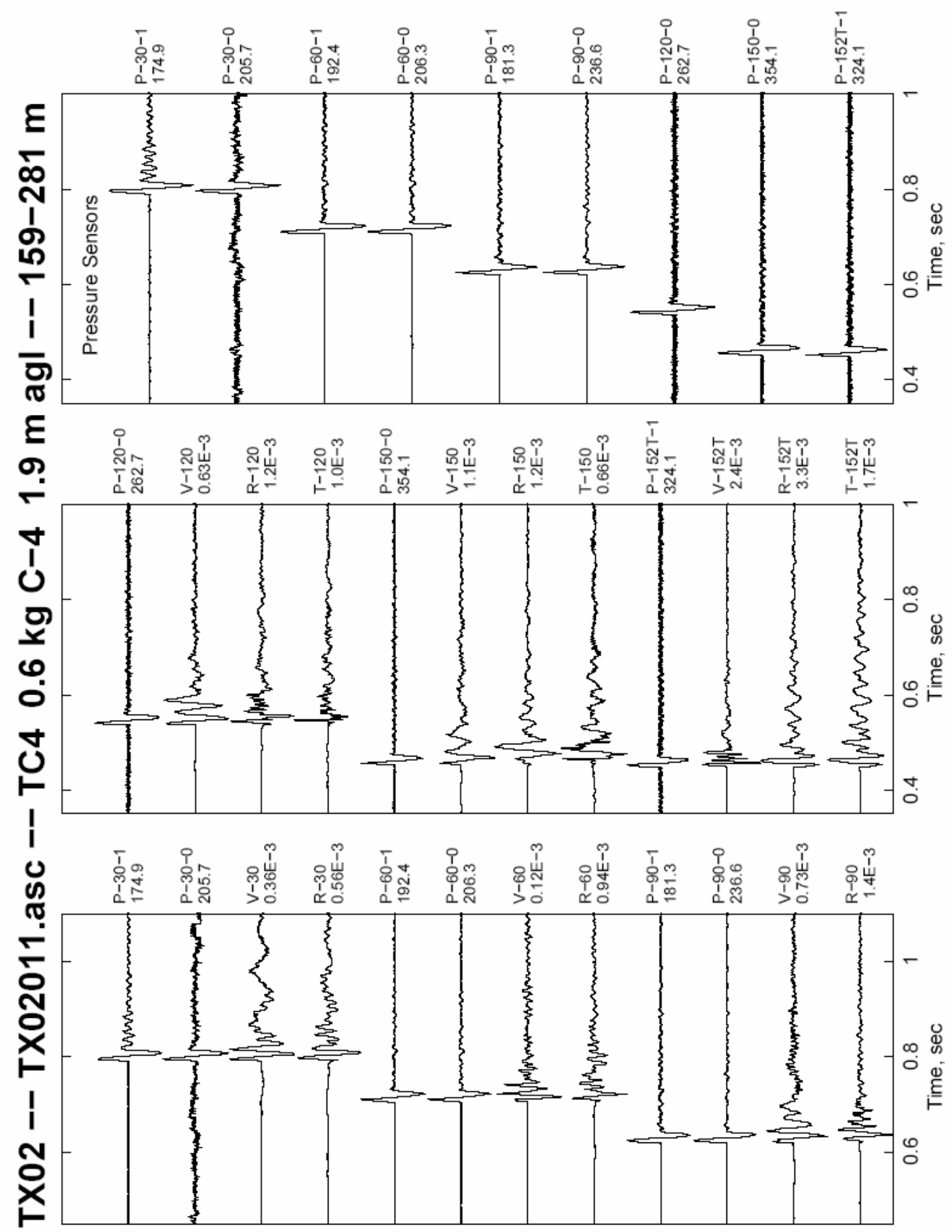




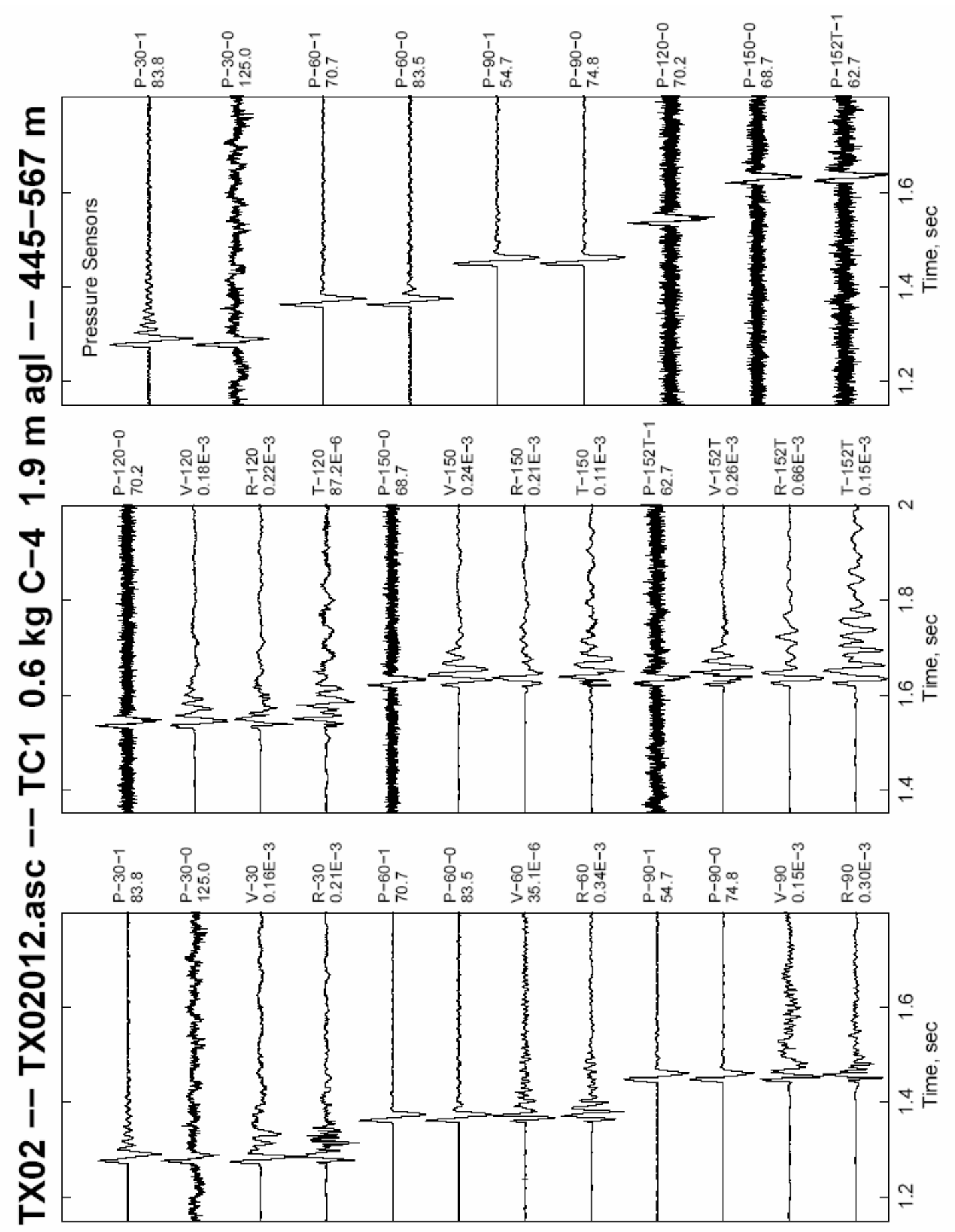




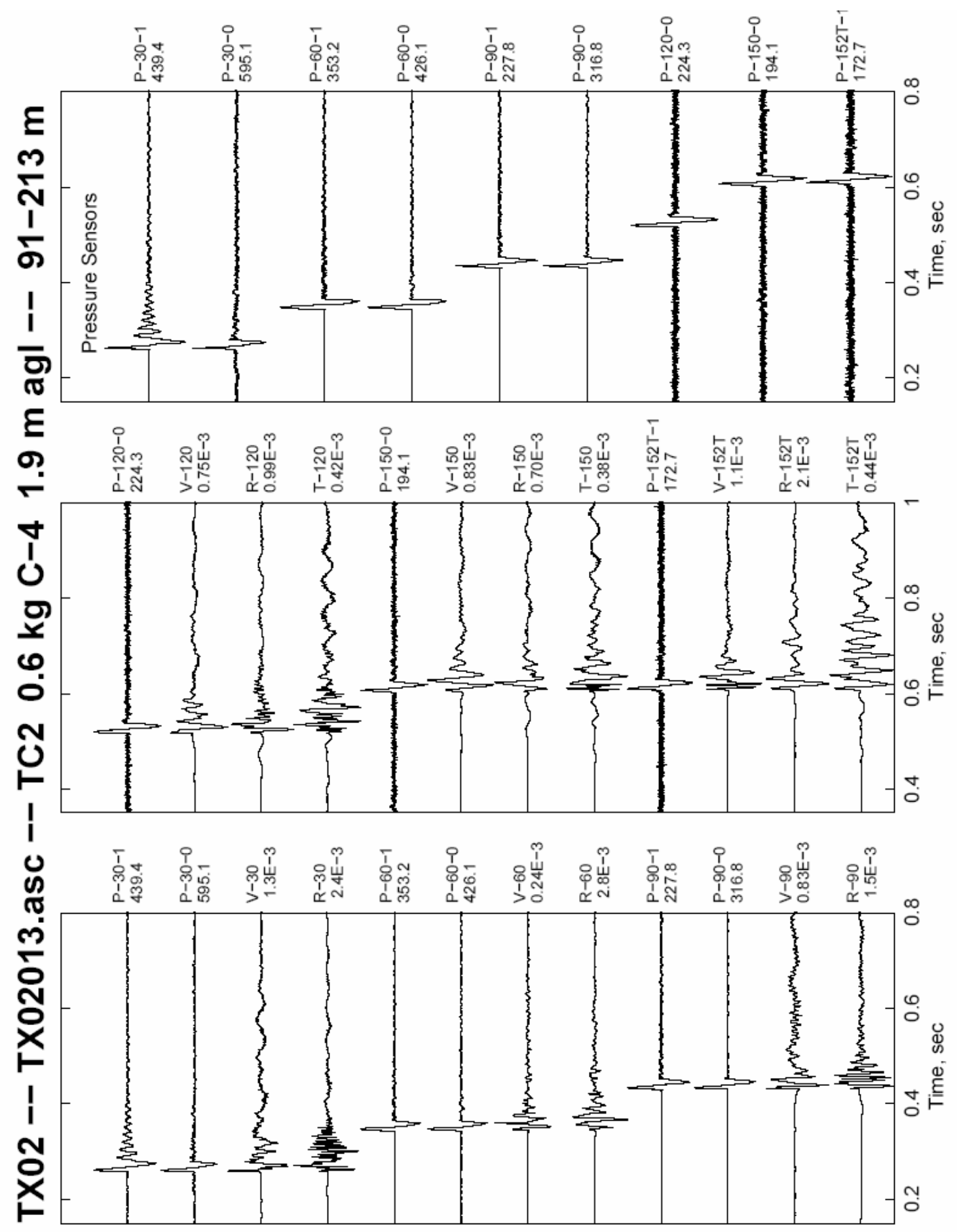




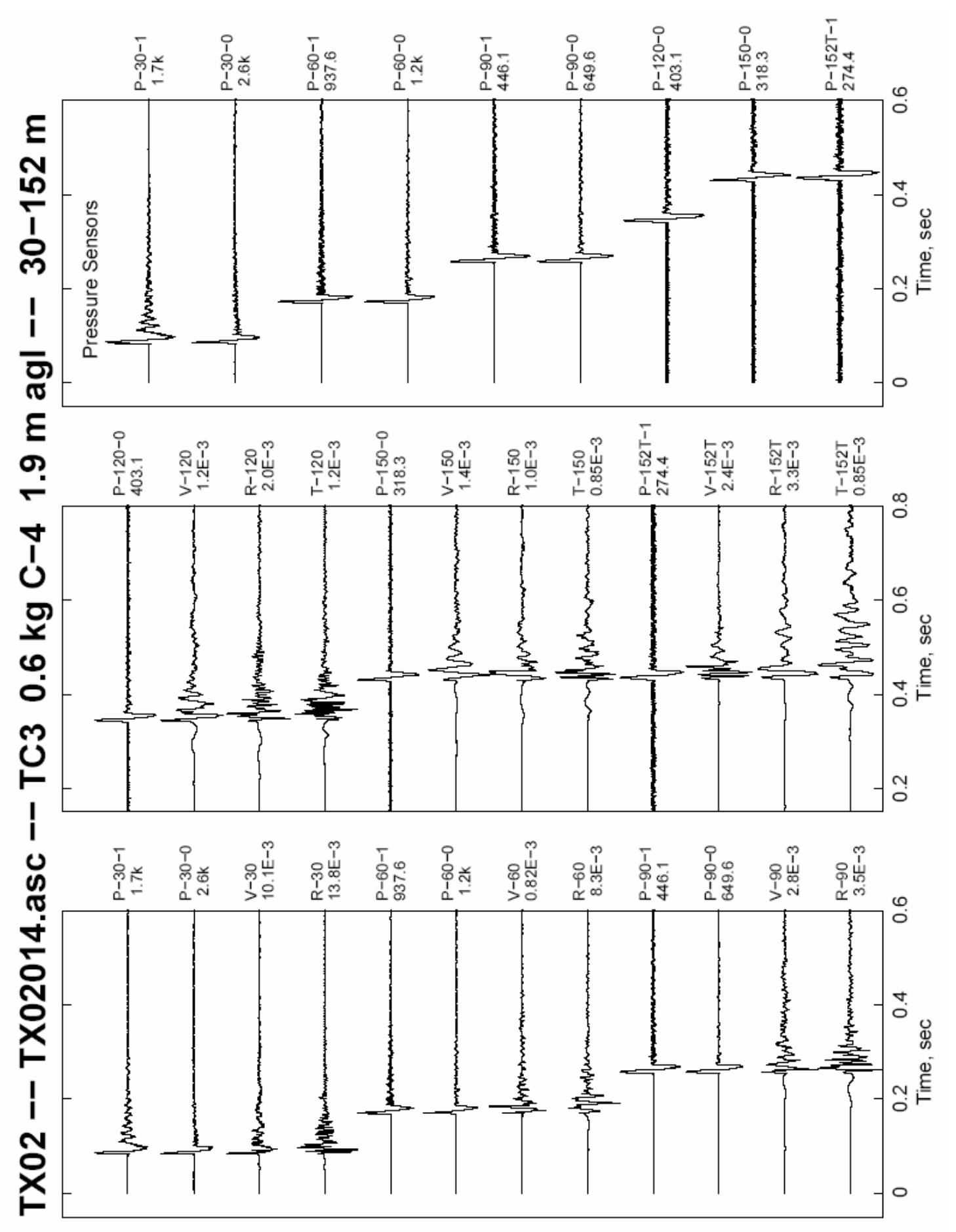




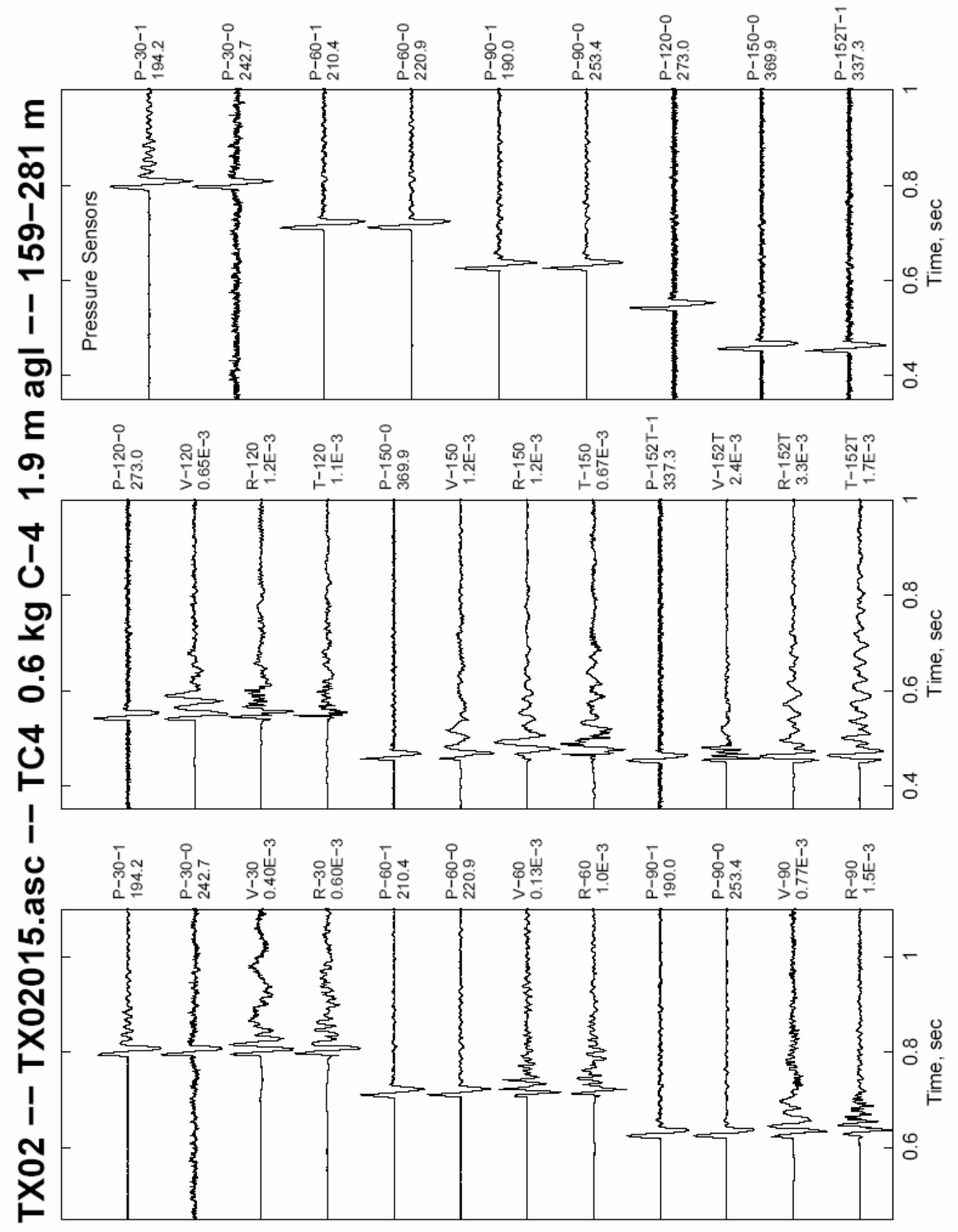




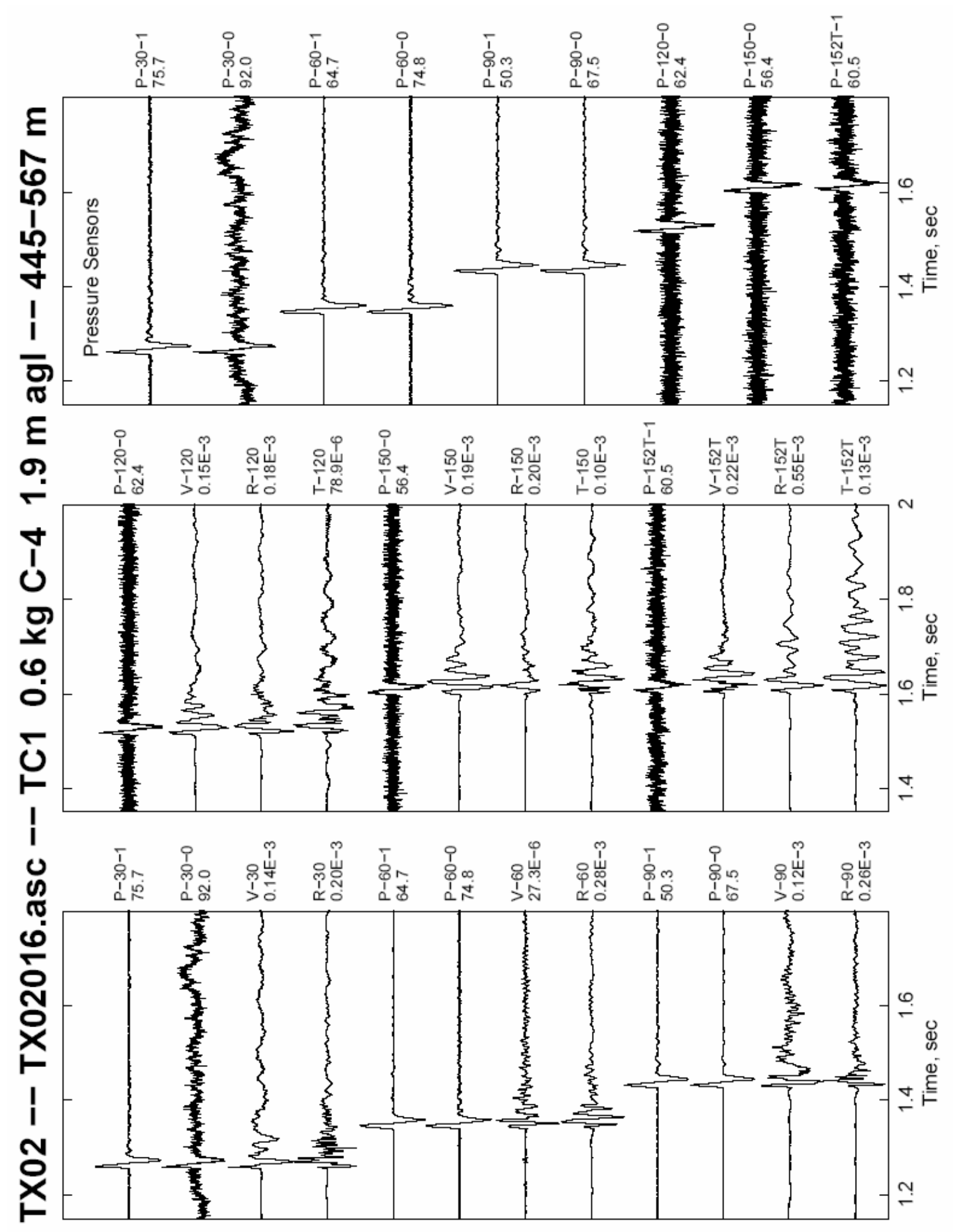




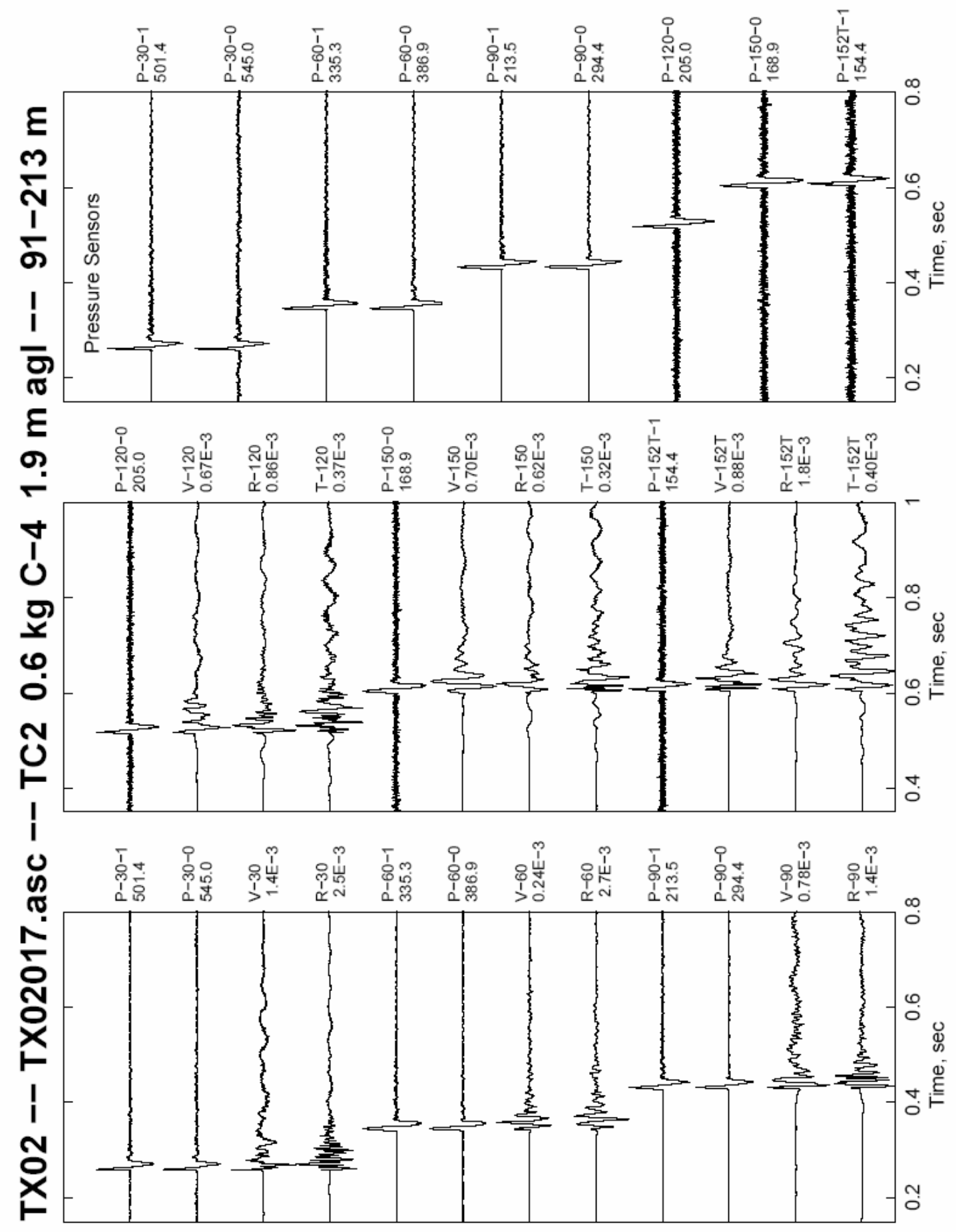




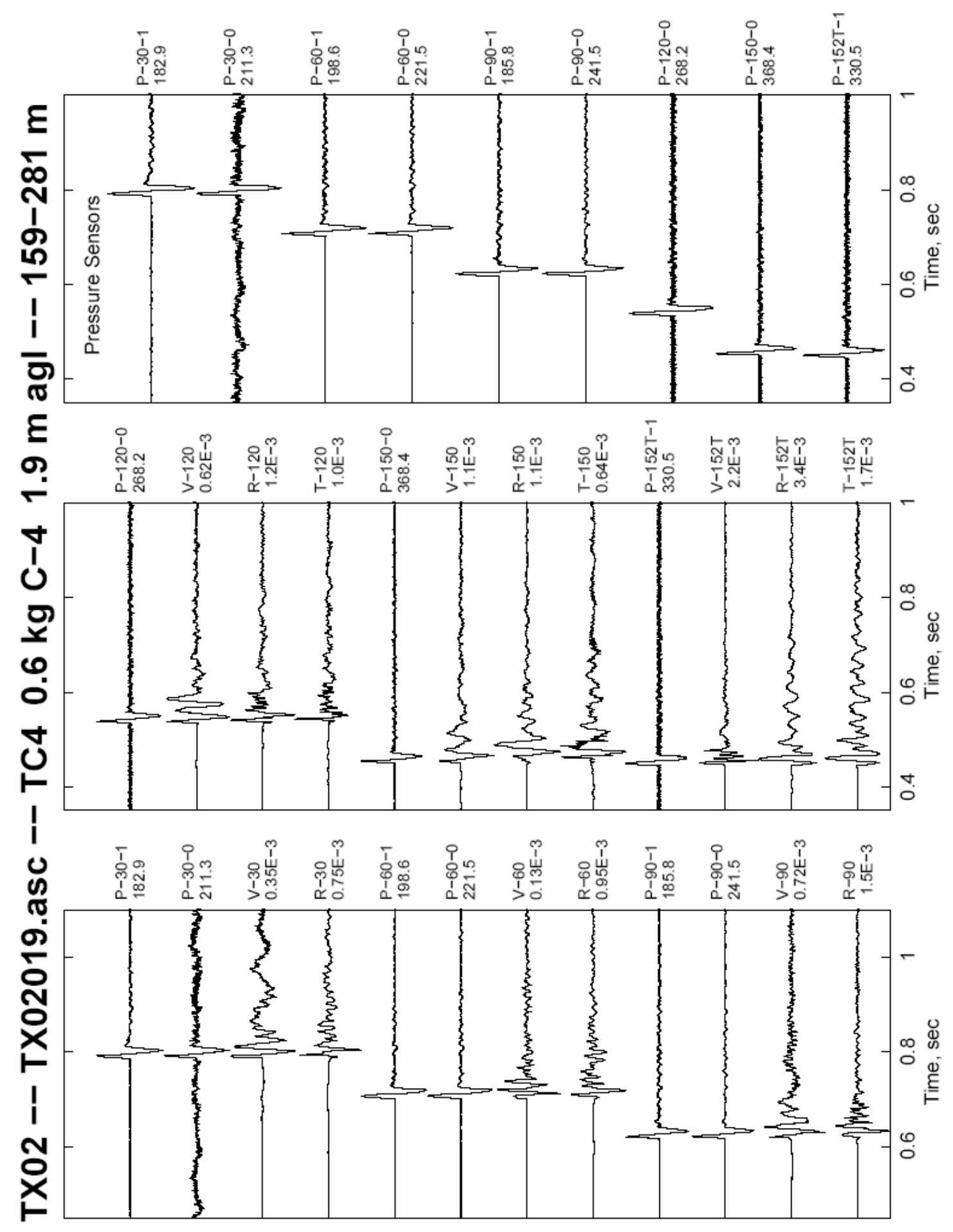




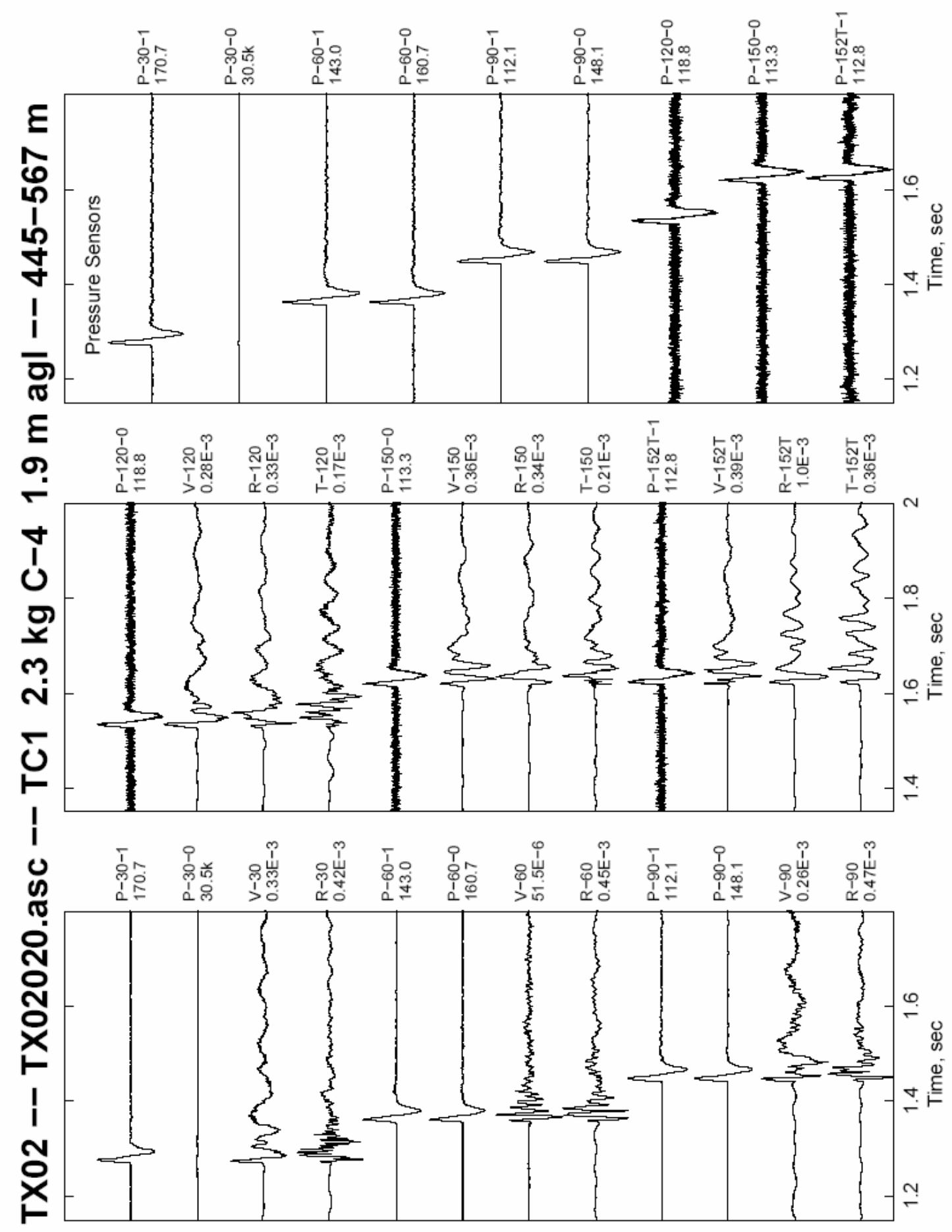




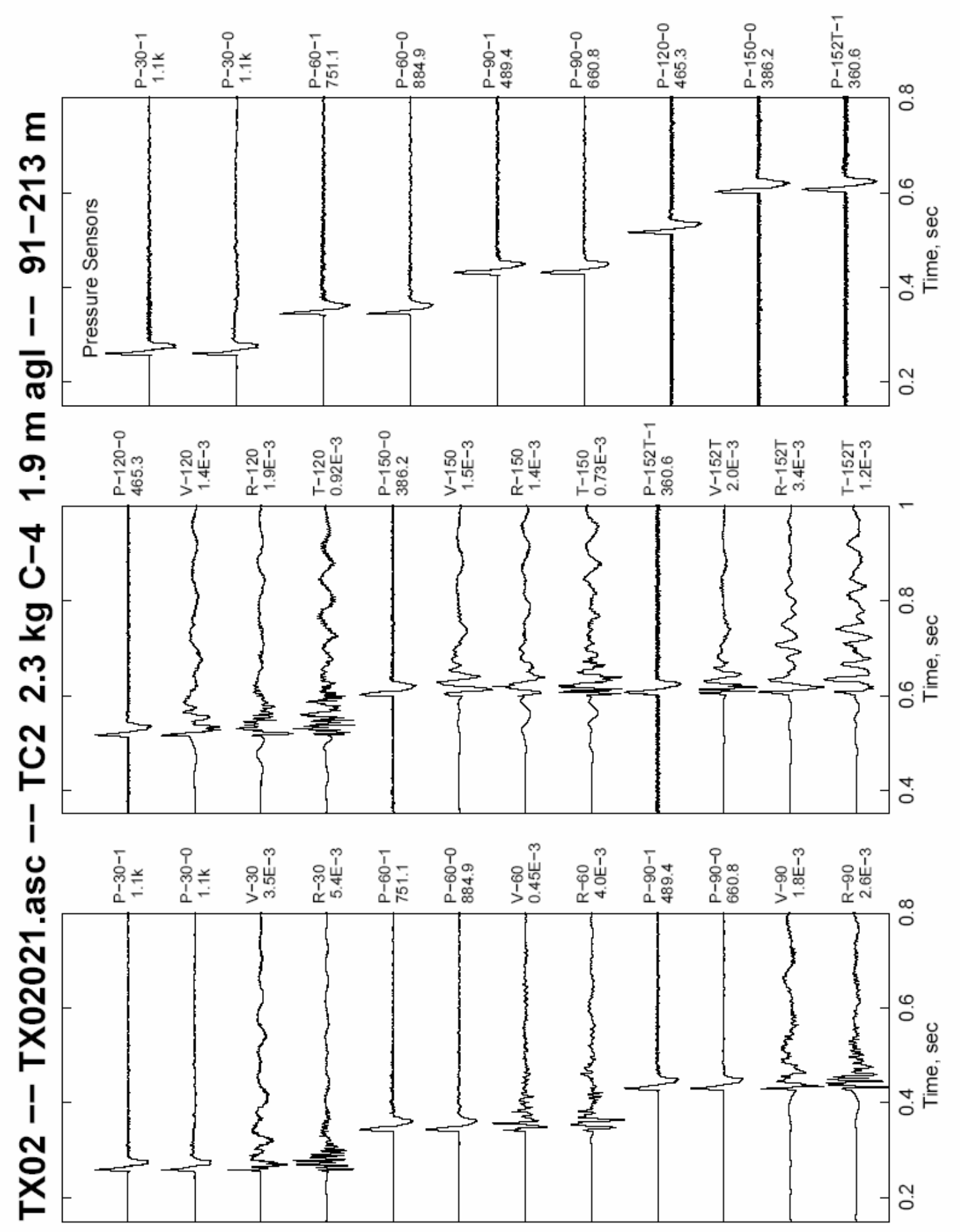




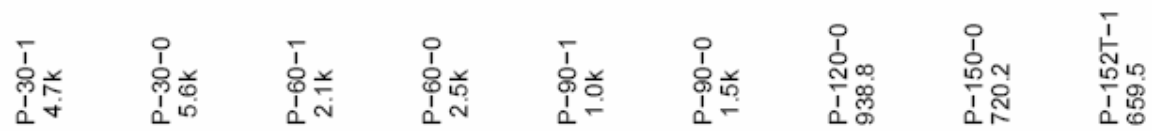

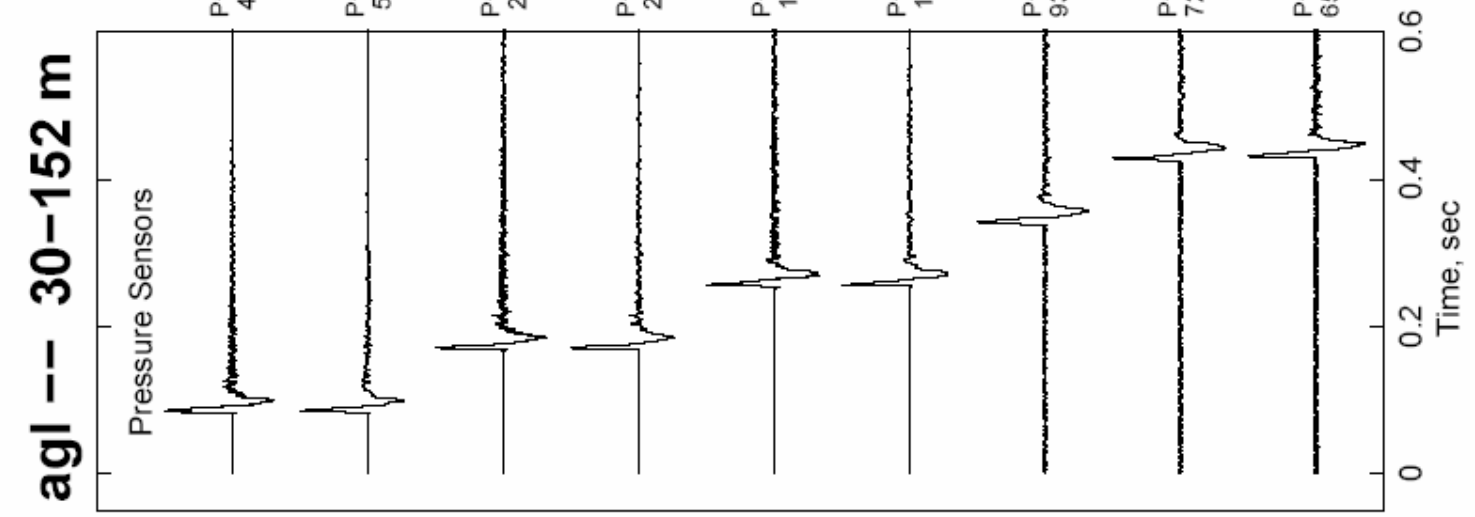

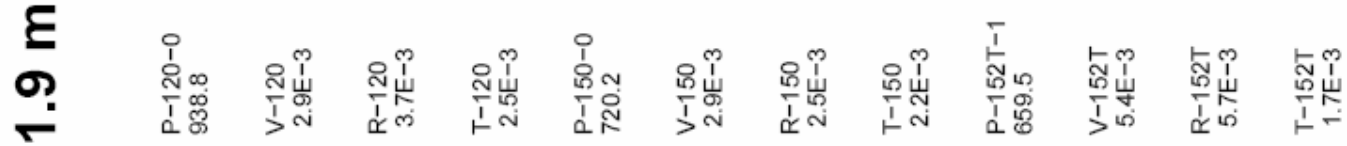

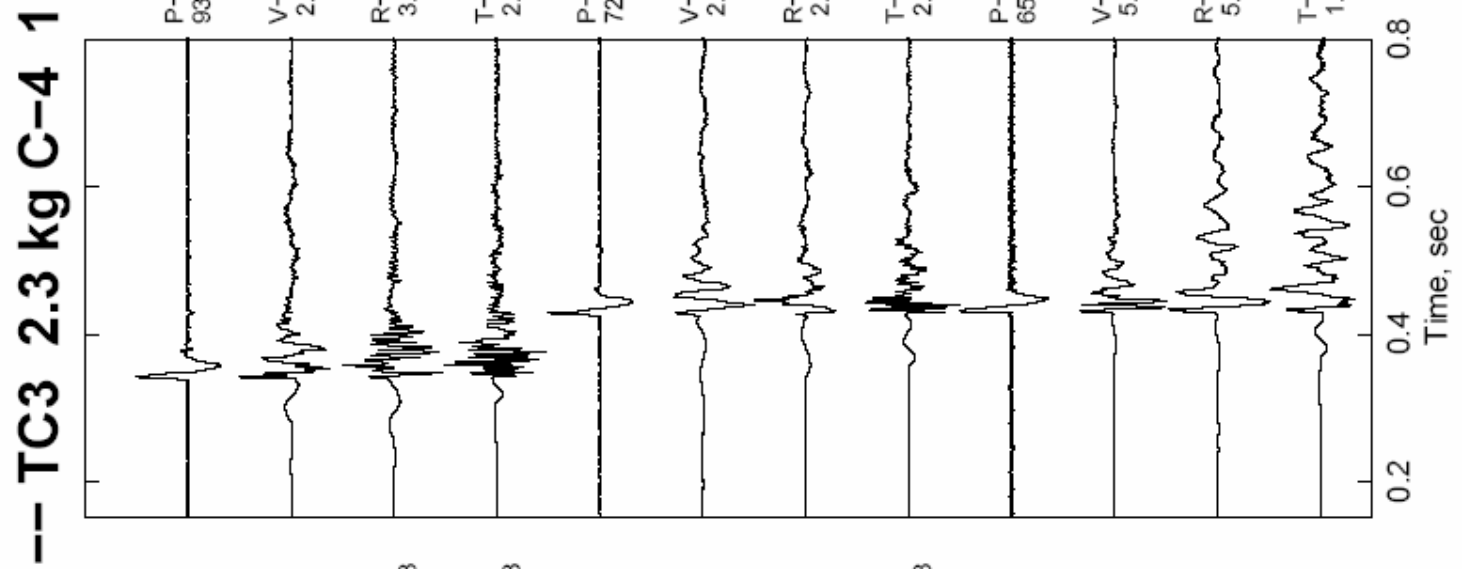

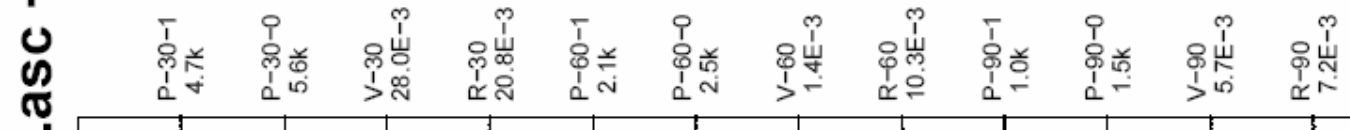

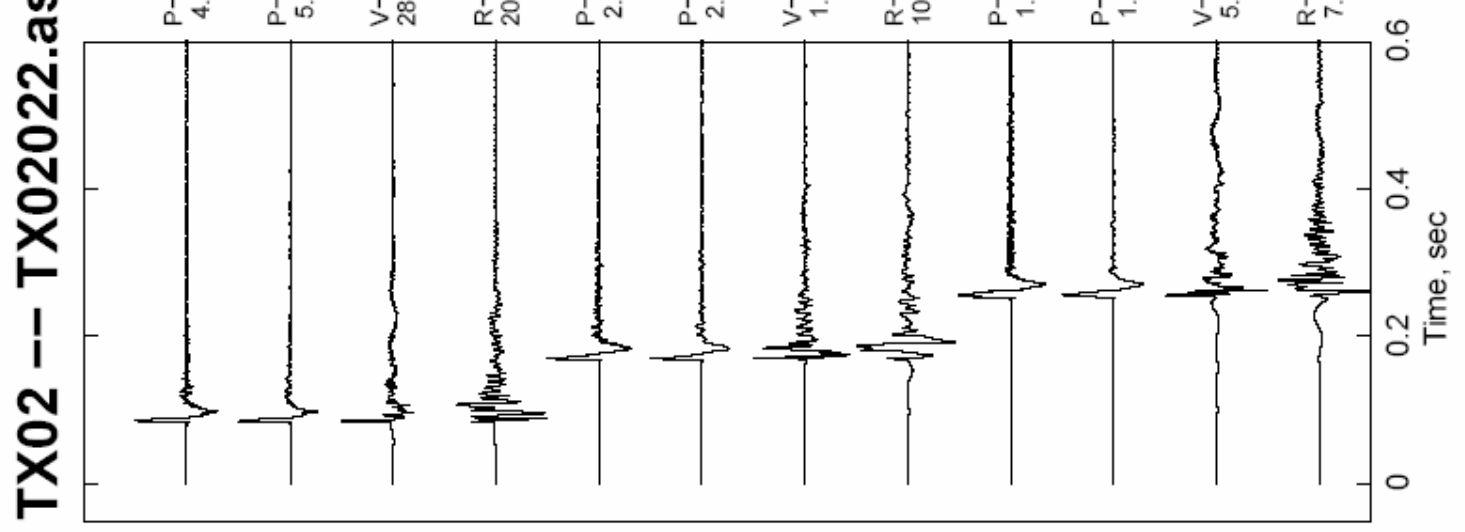




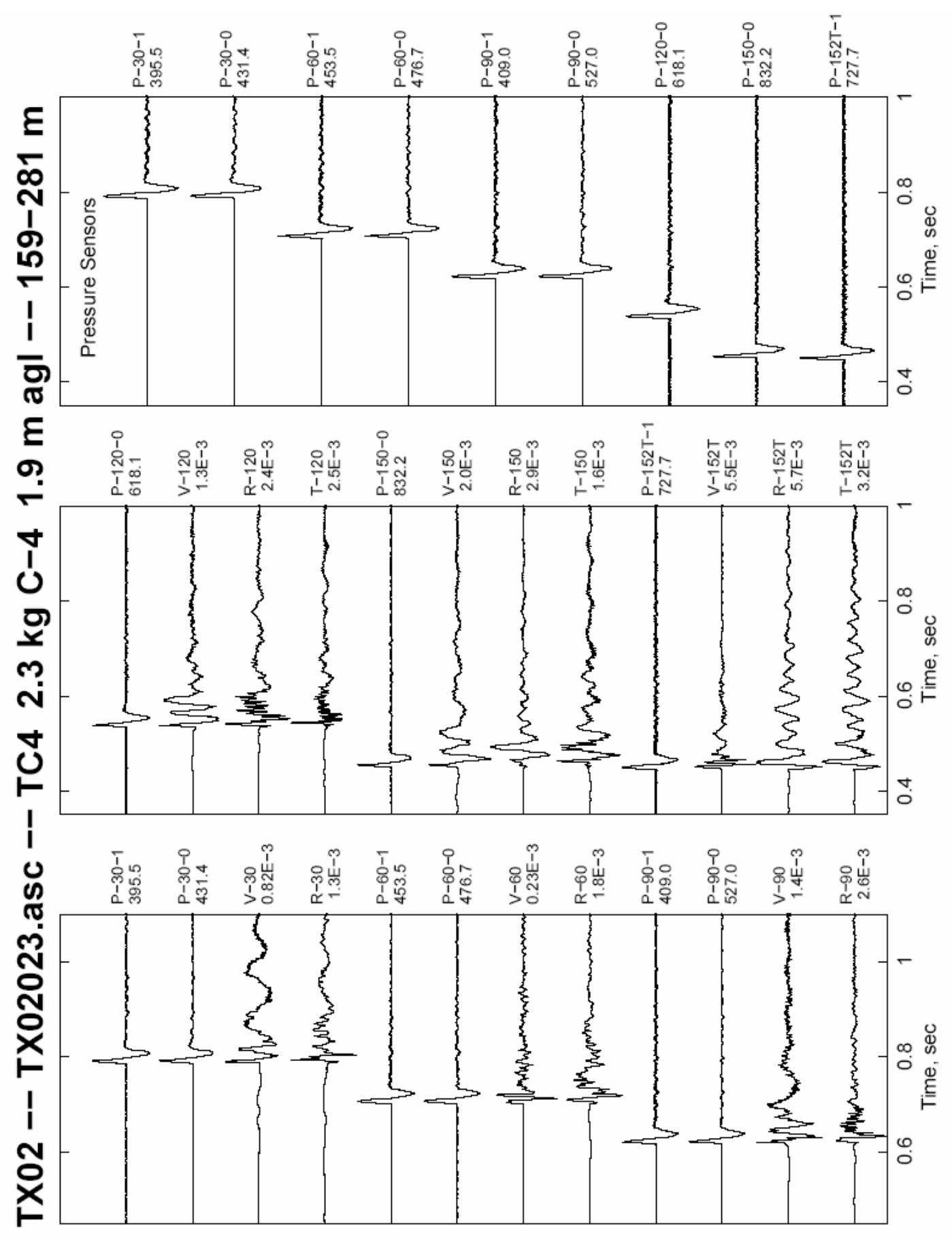




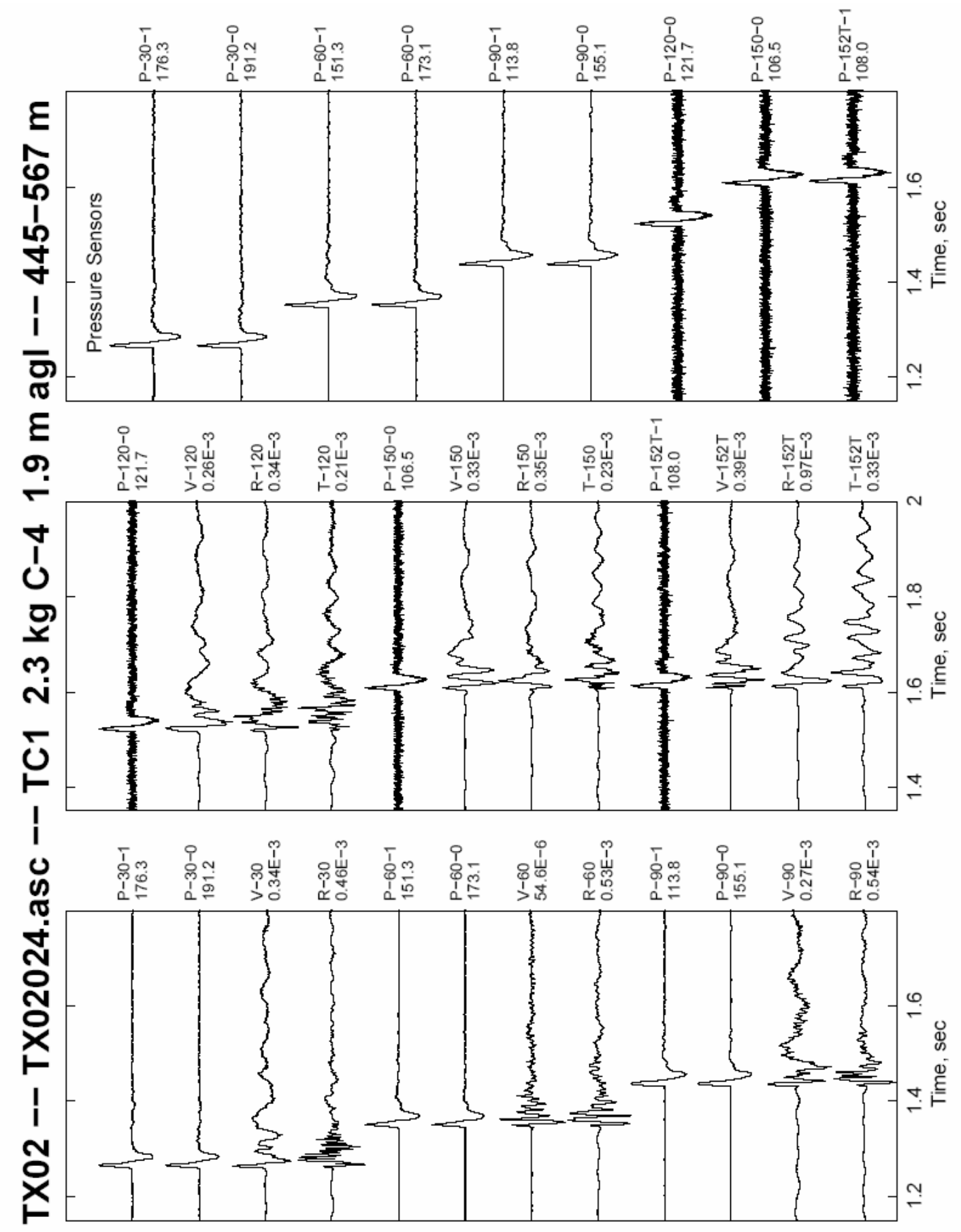




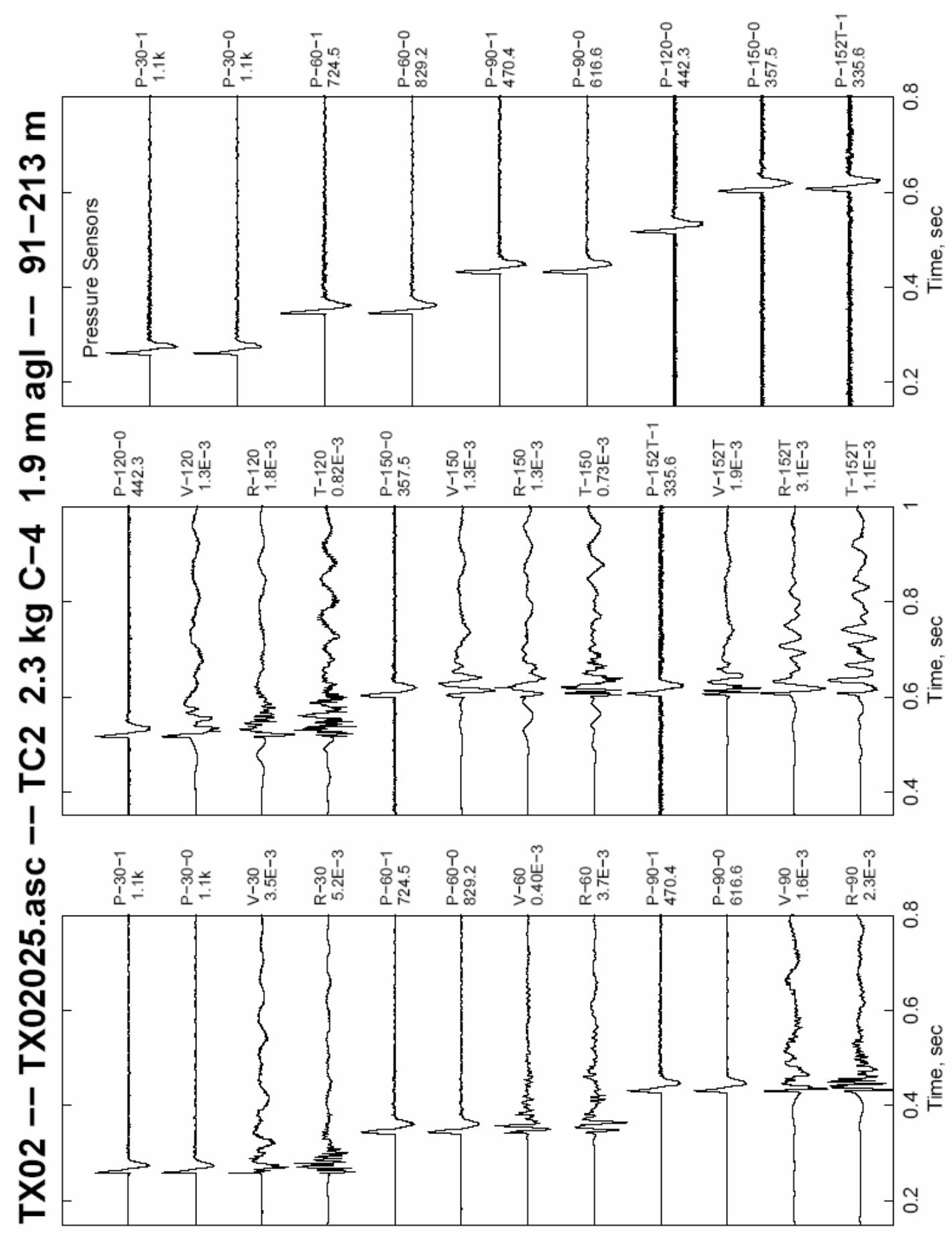




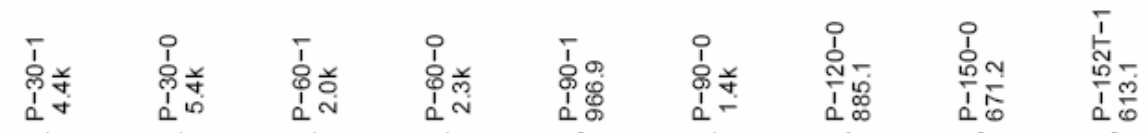

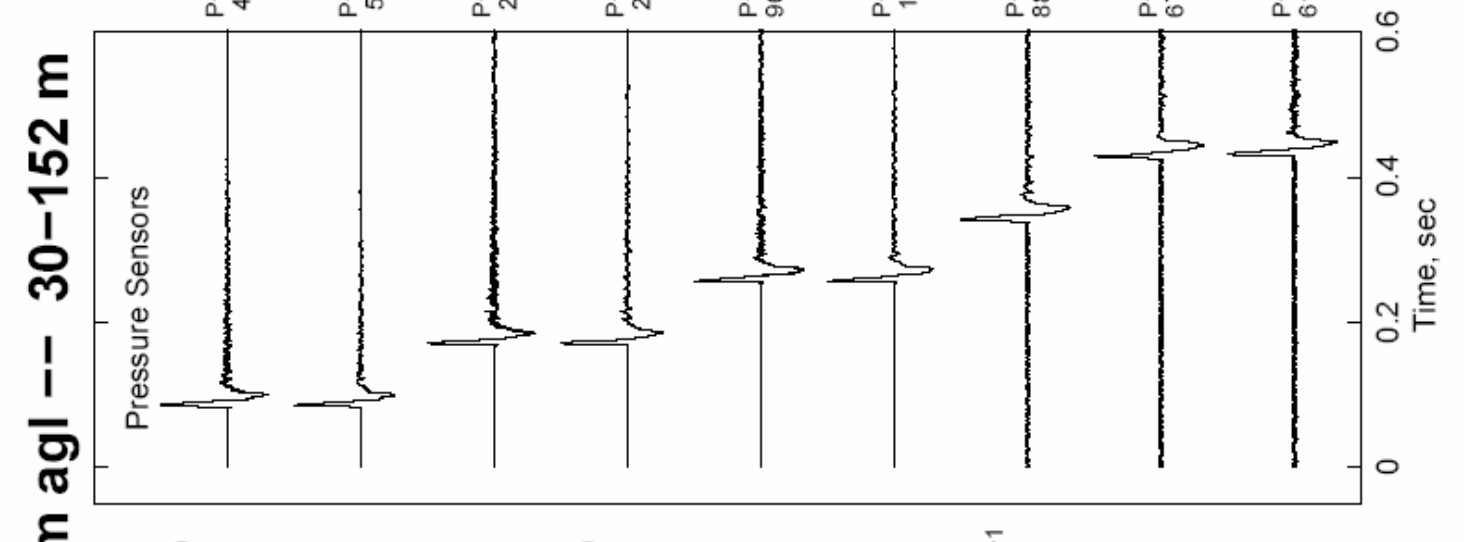

E)

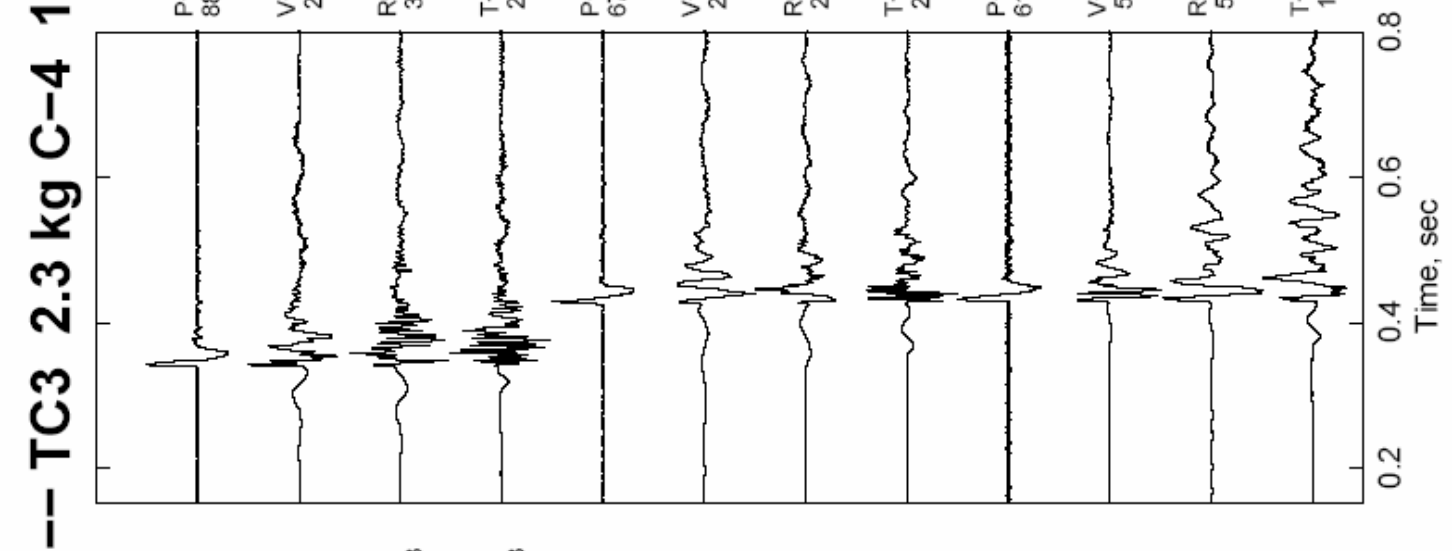

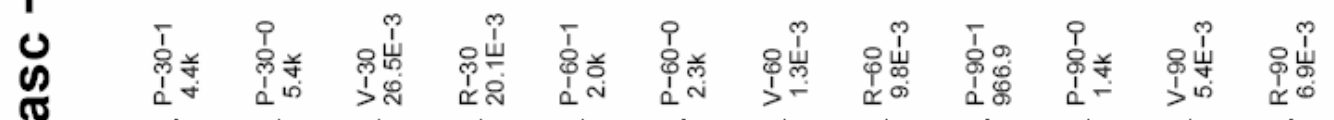

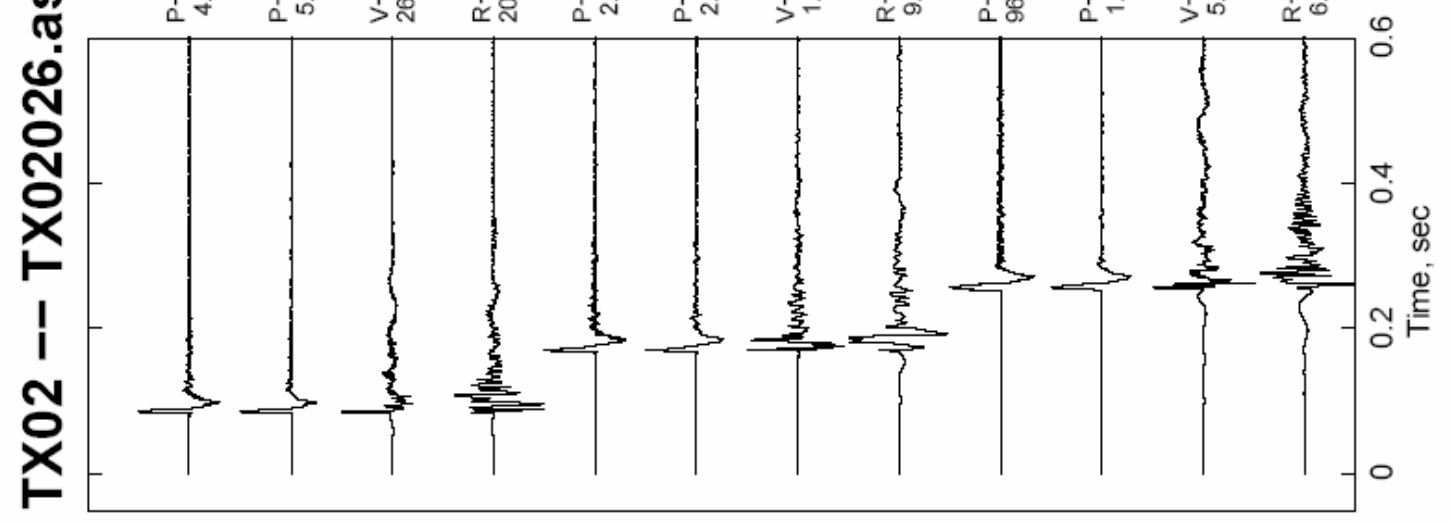




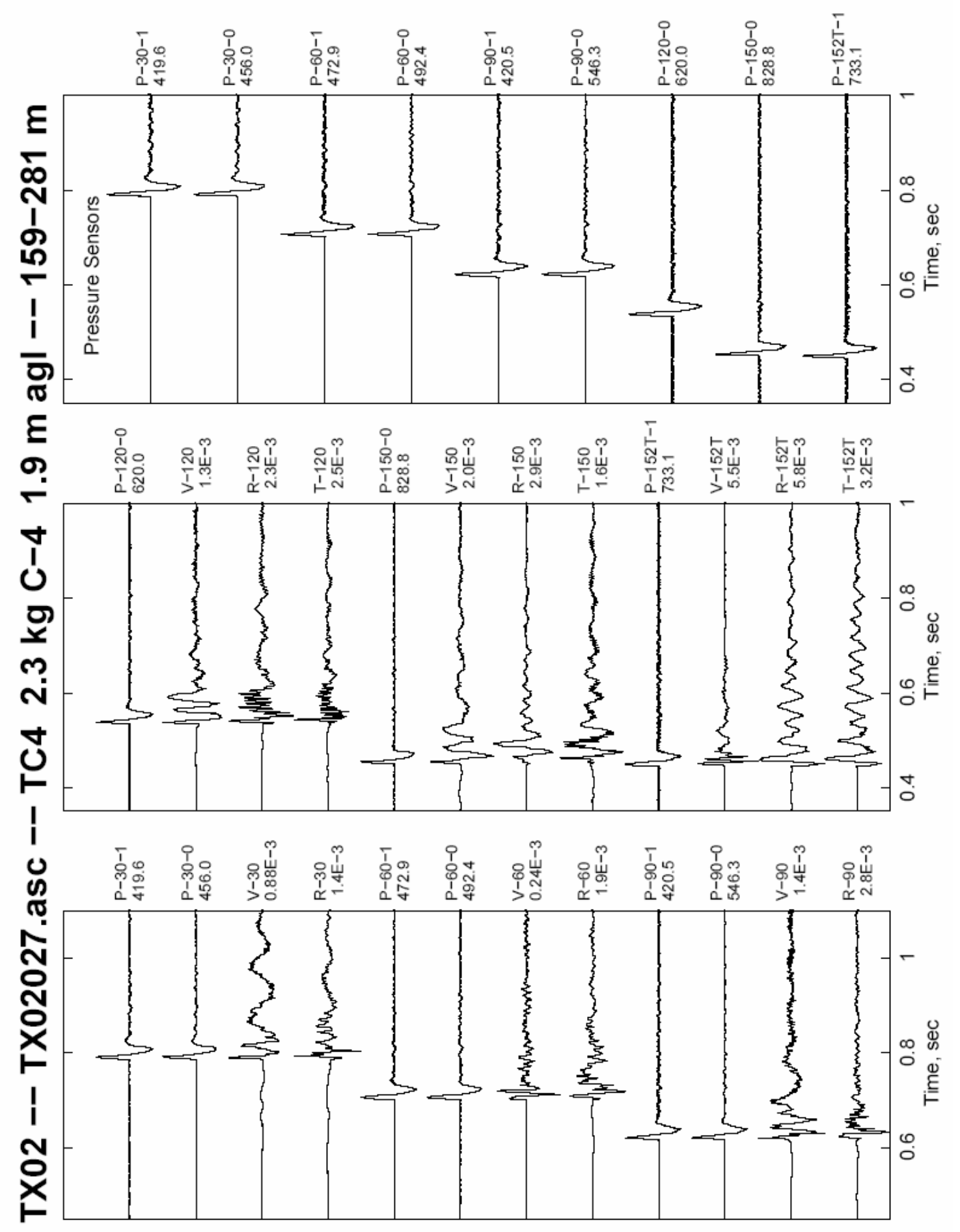




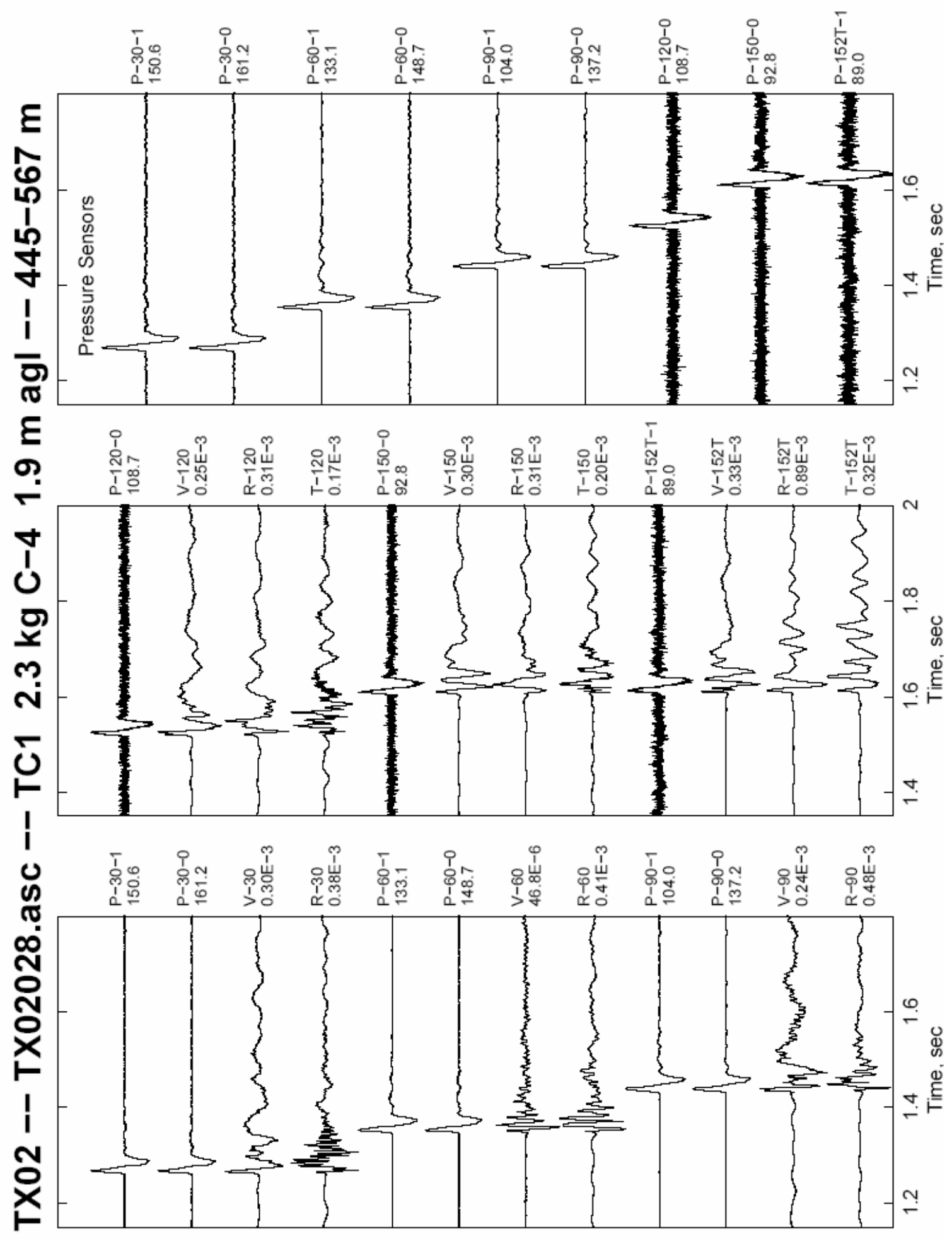




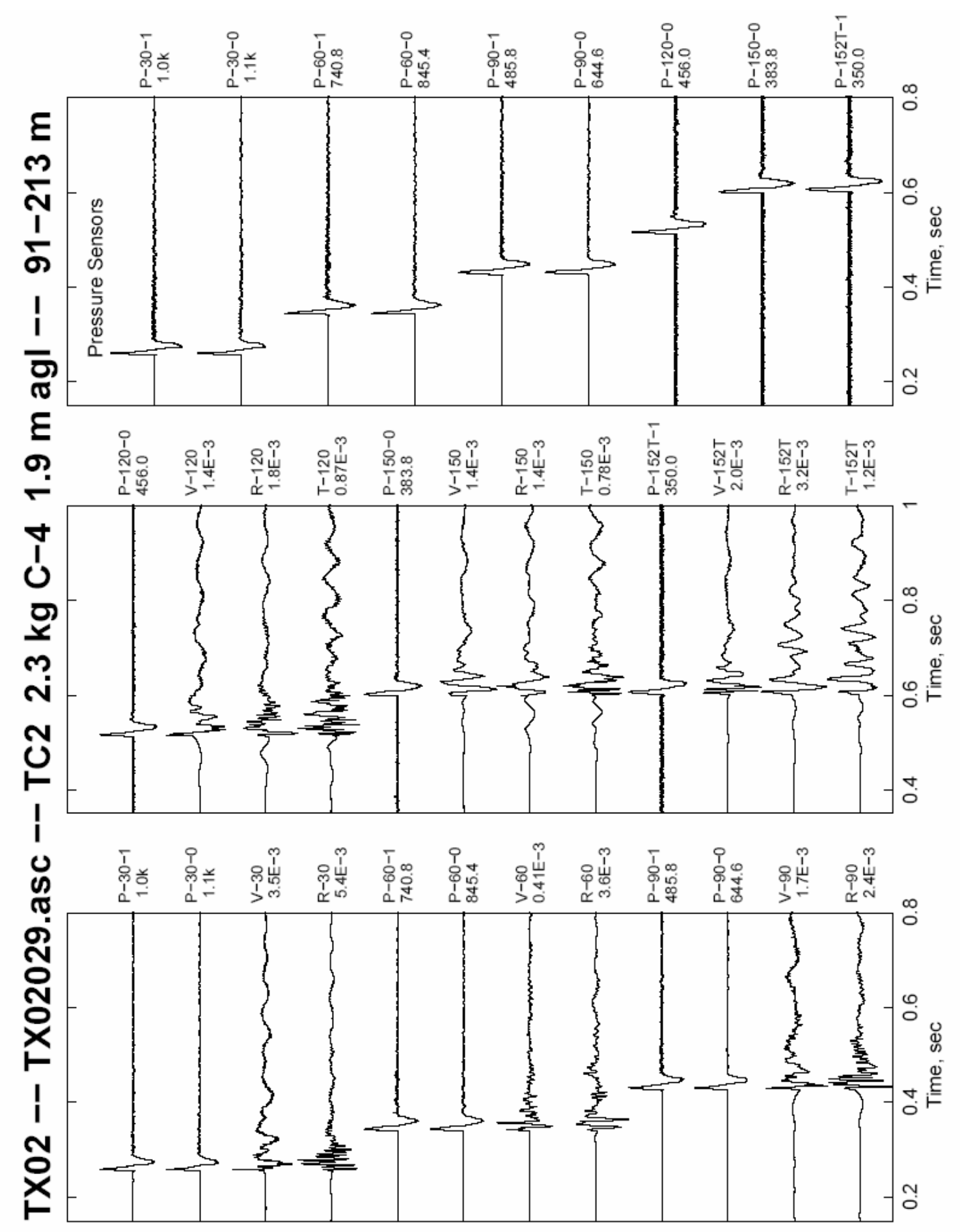




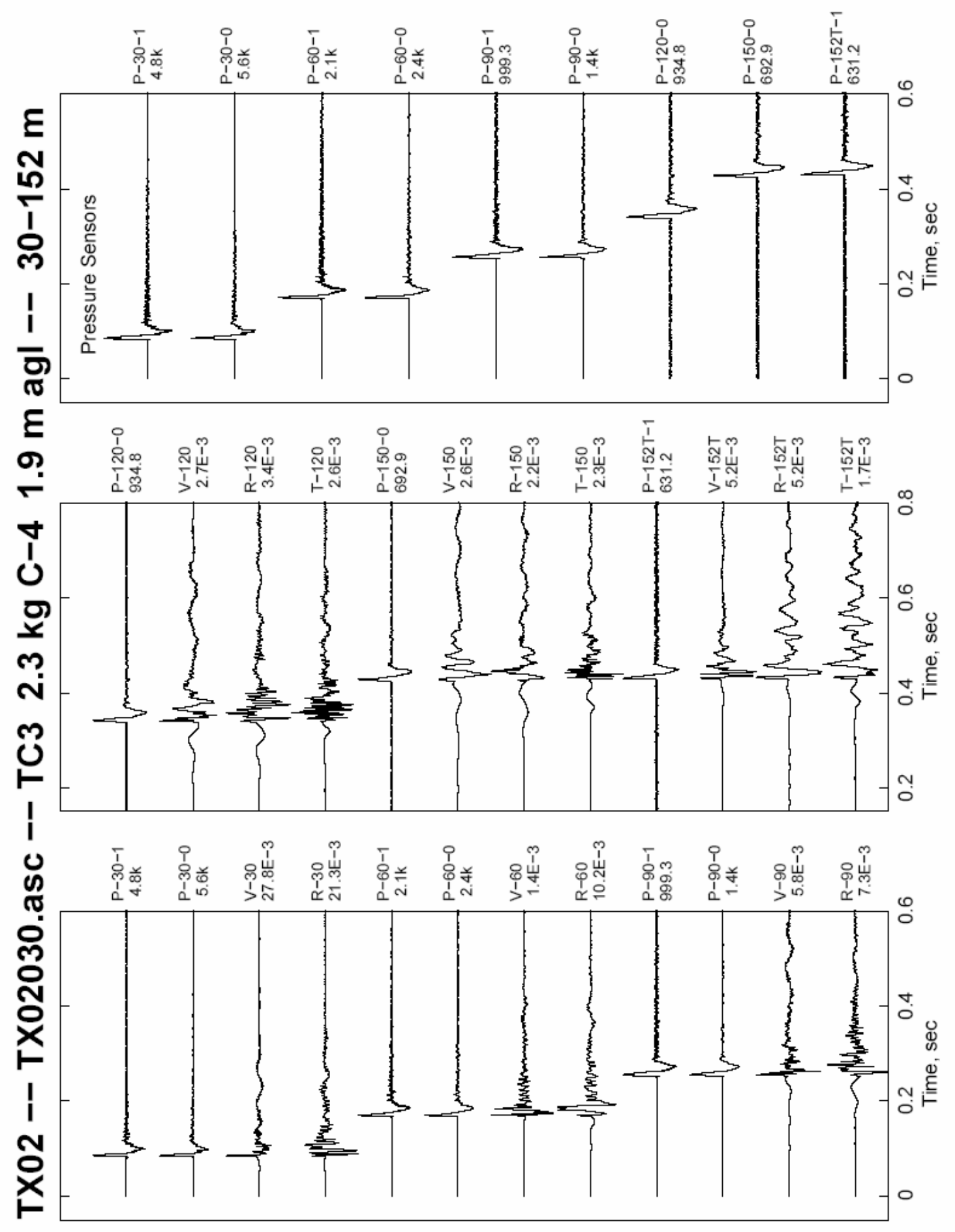




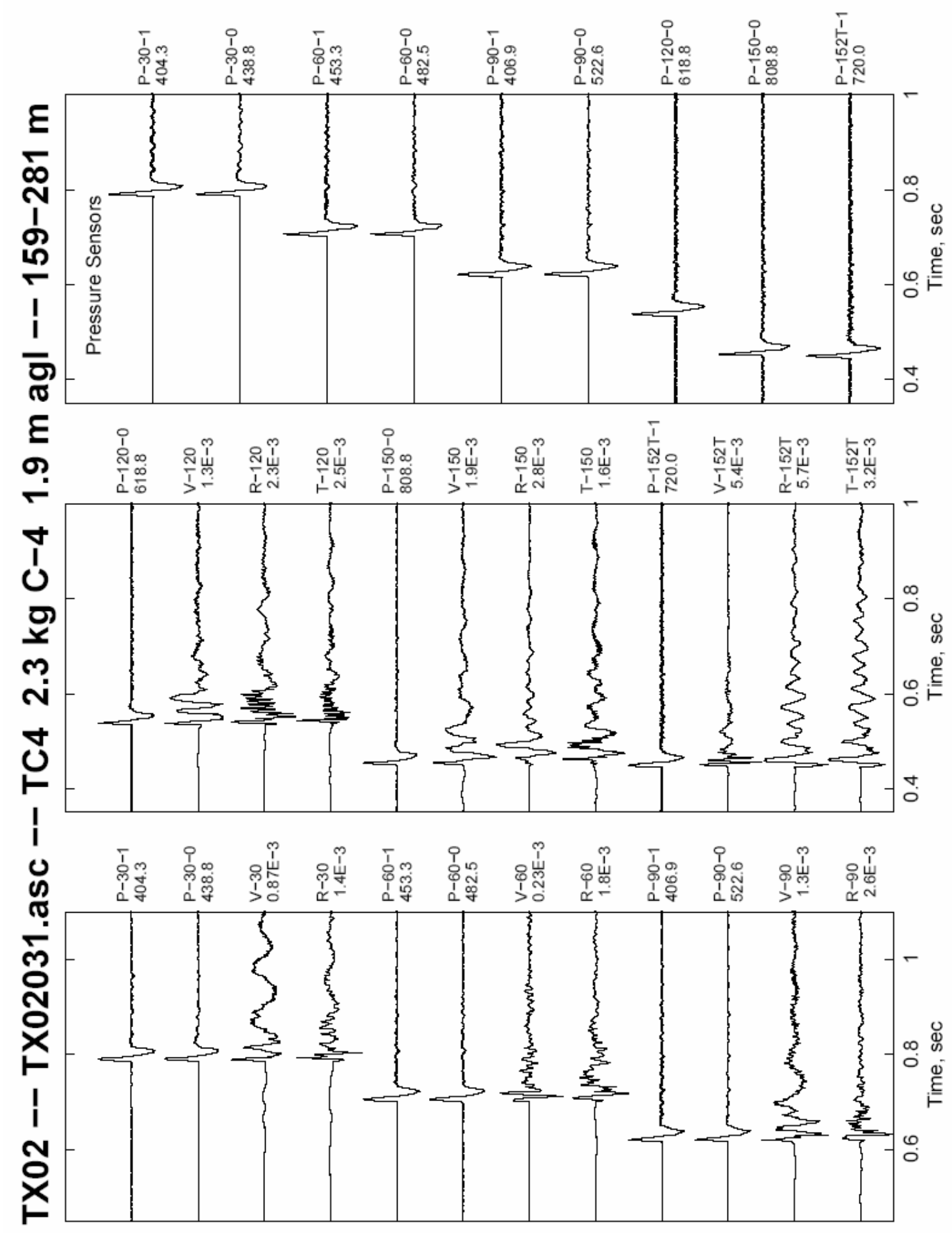




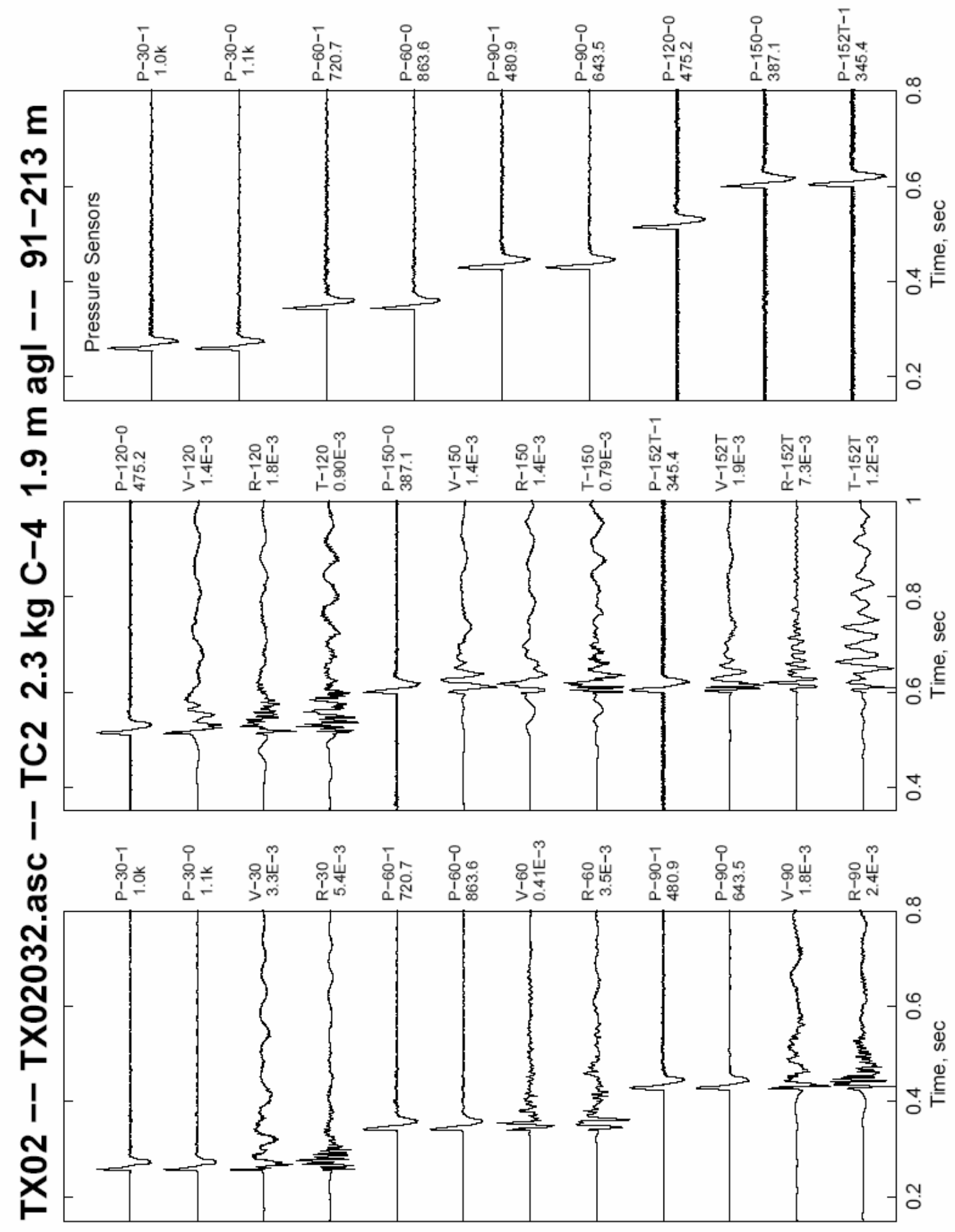




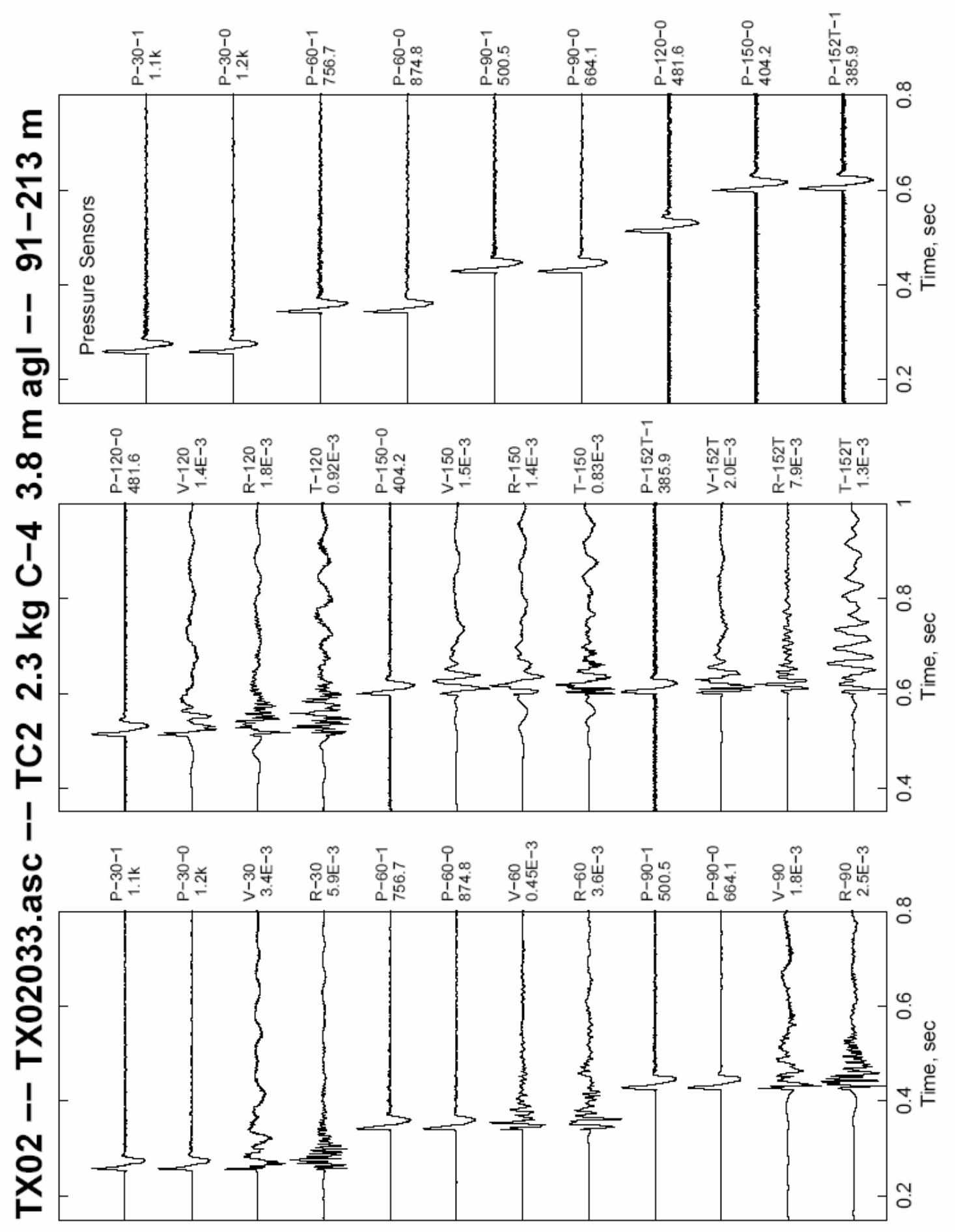




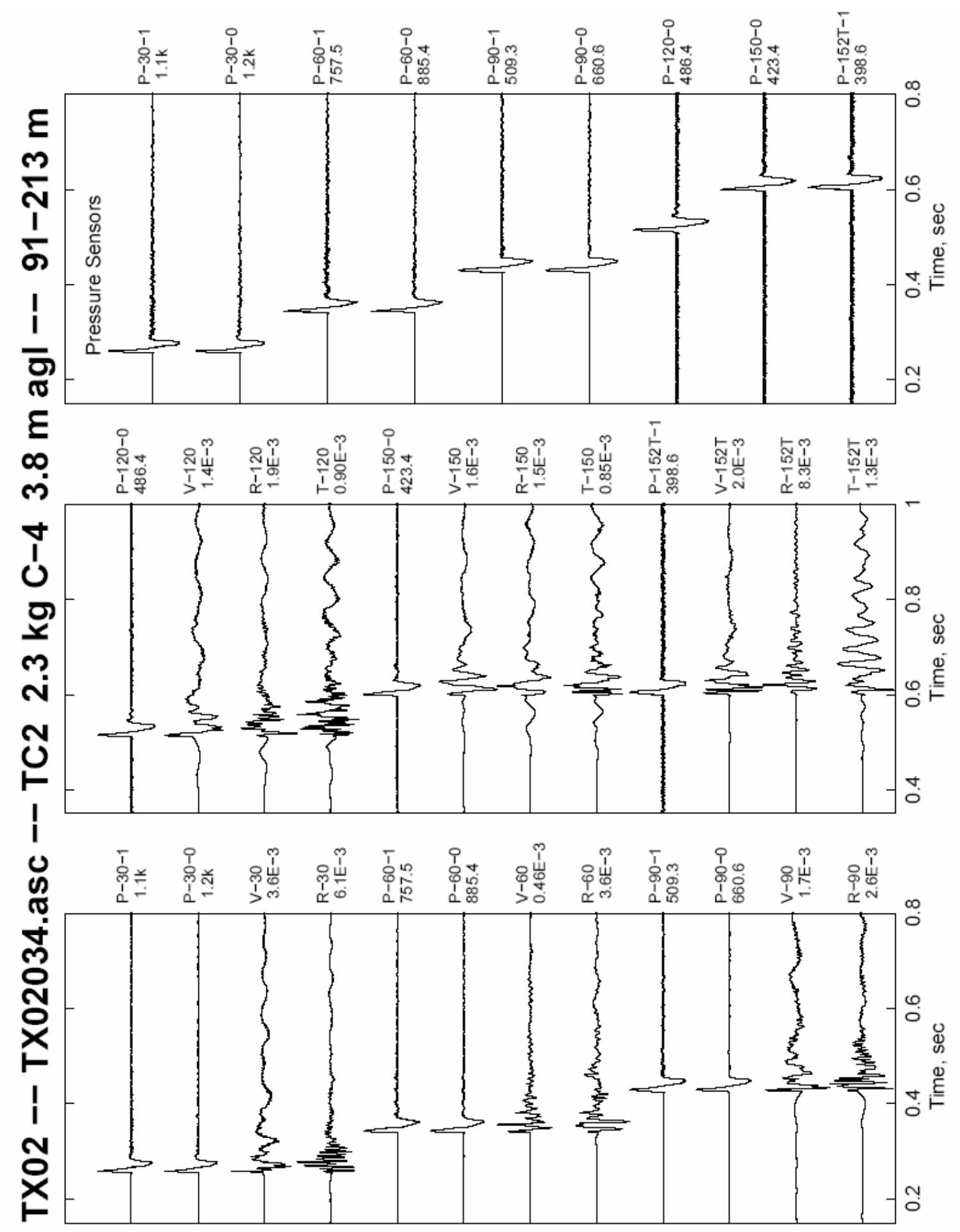




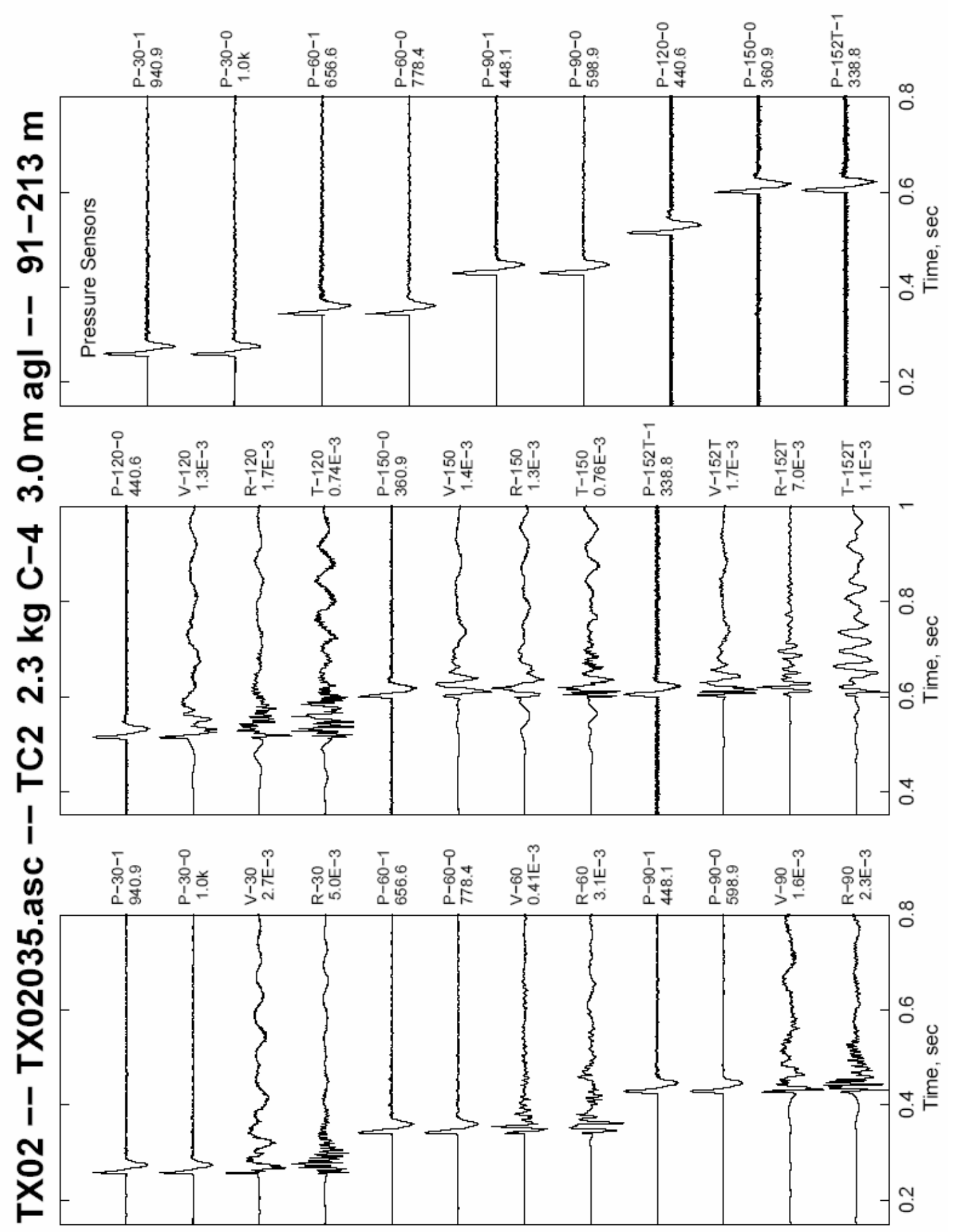




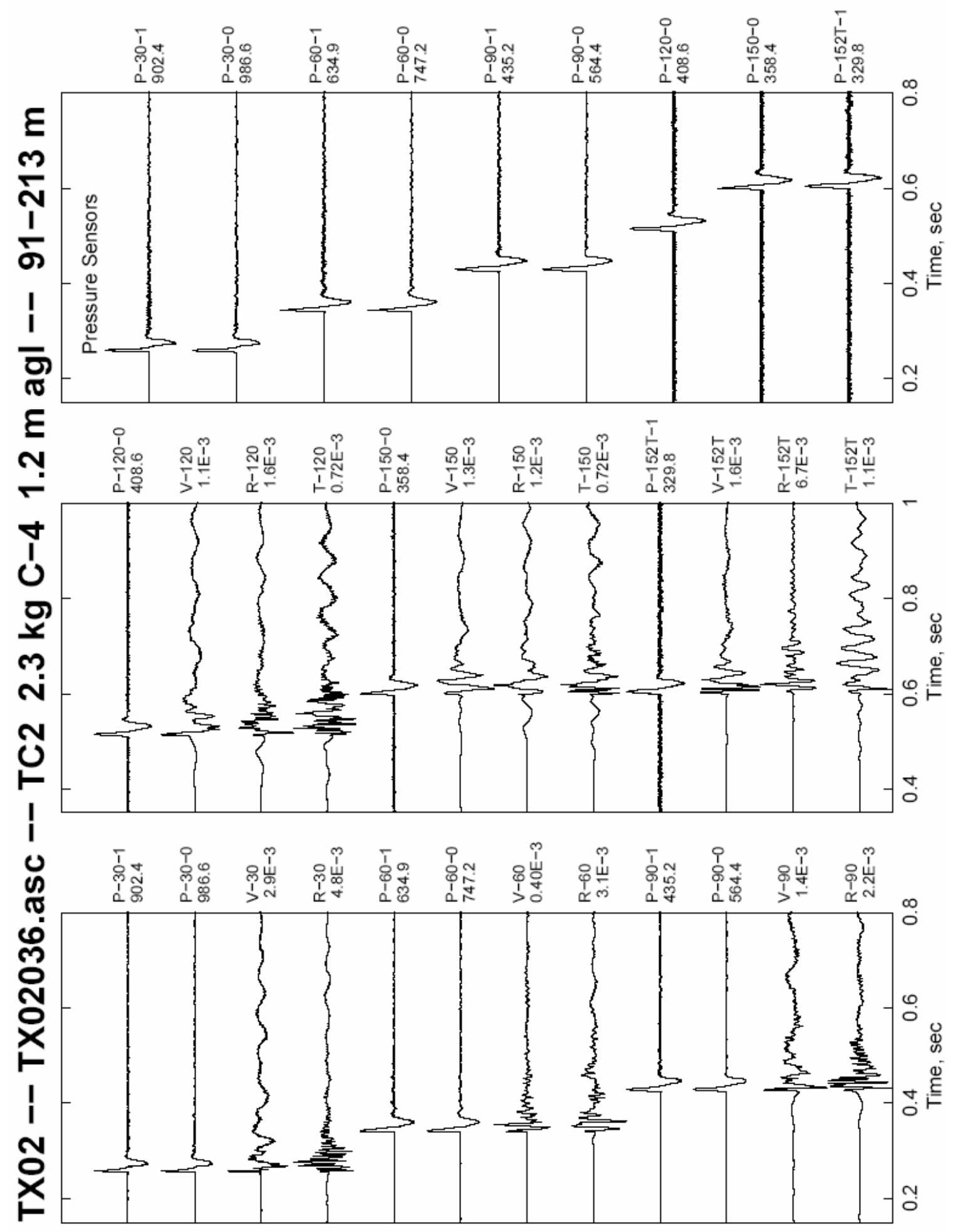




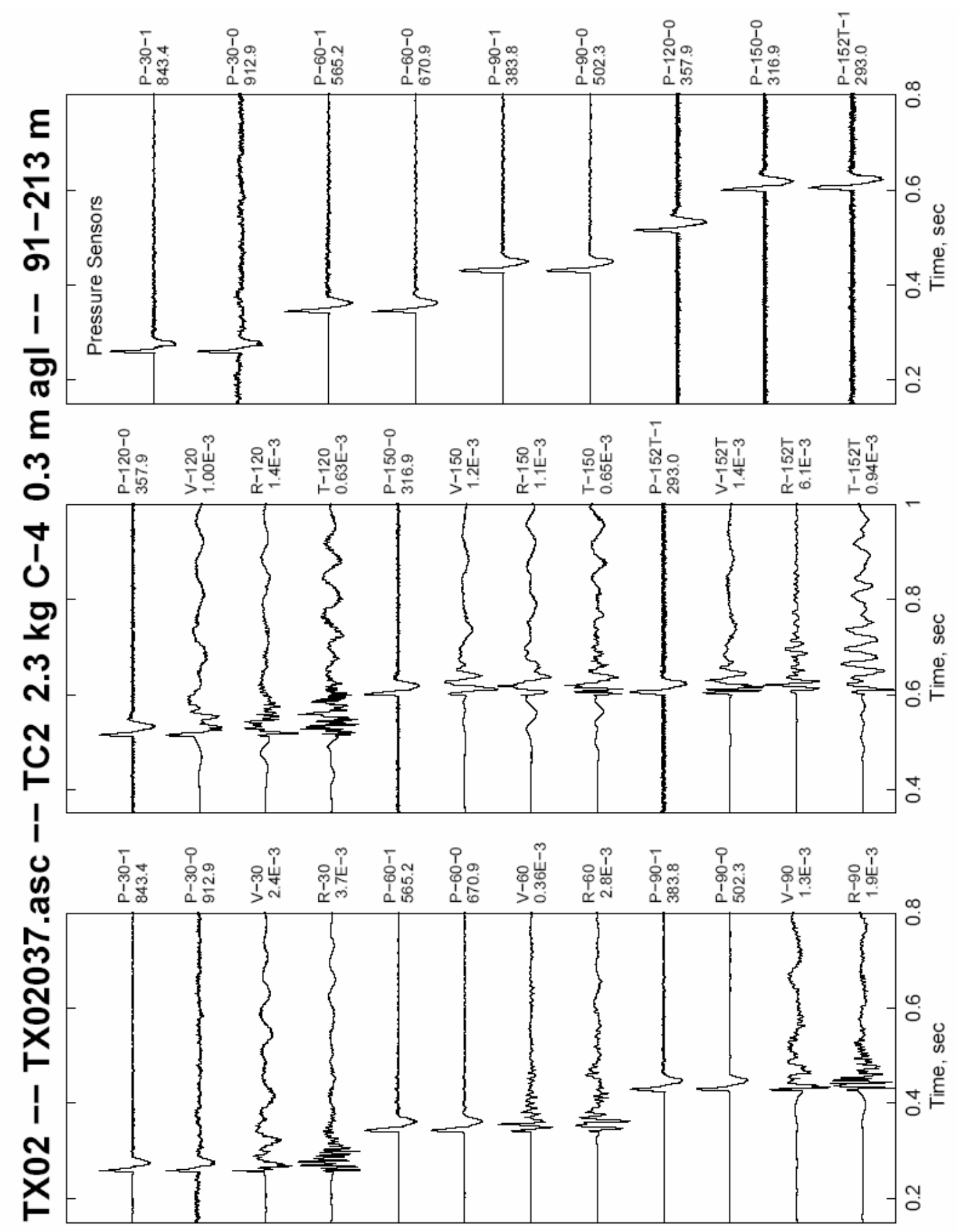




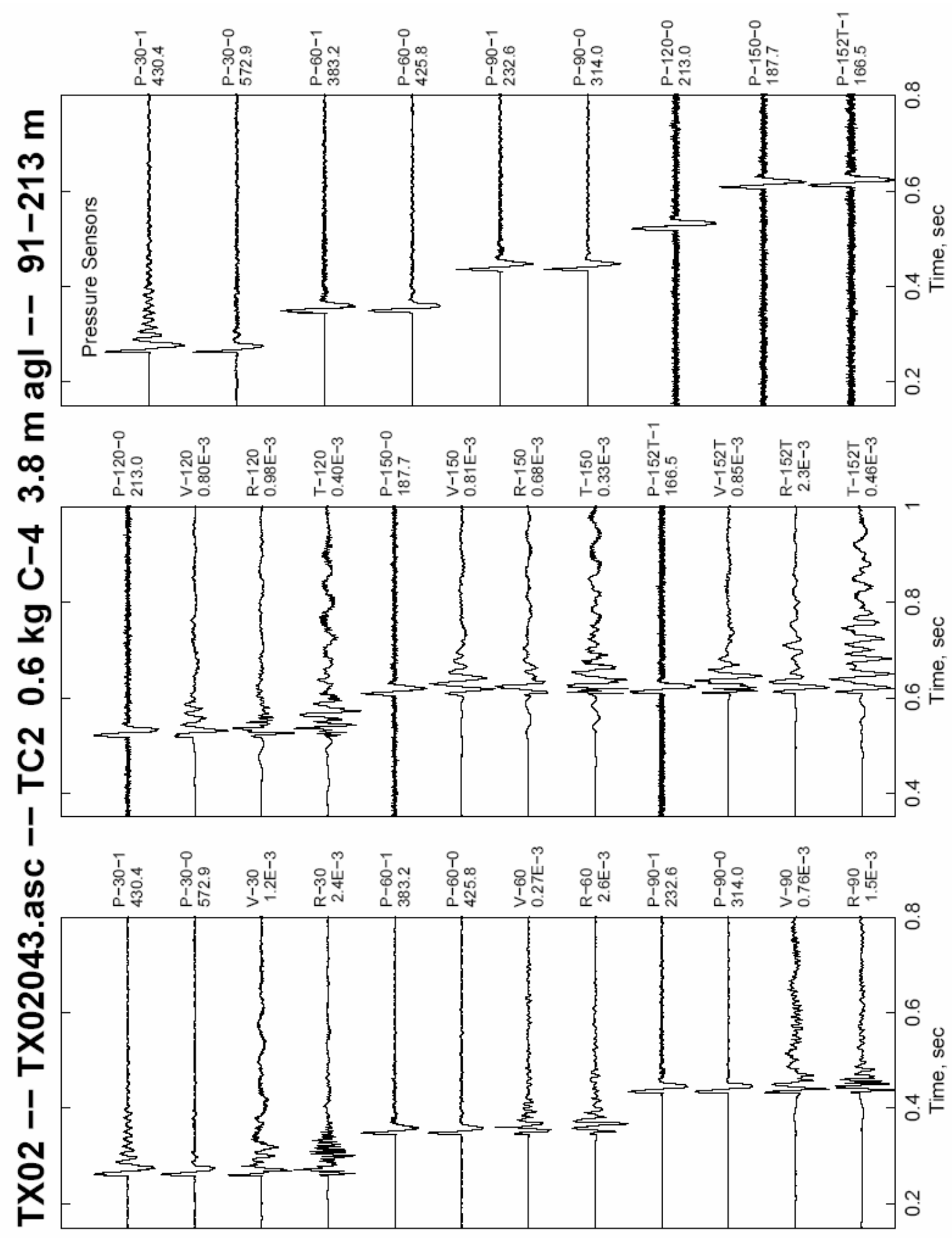




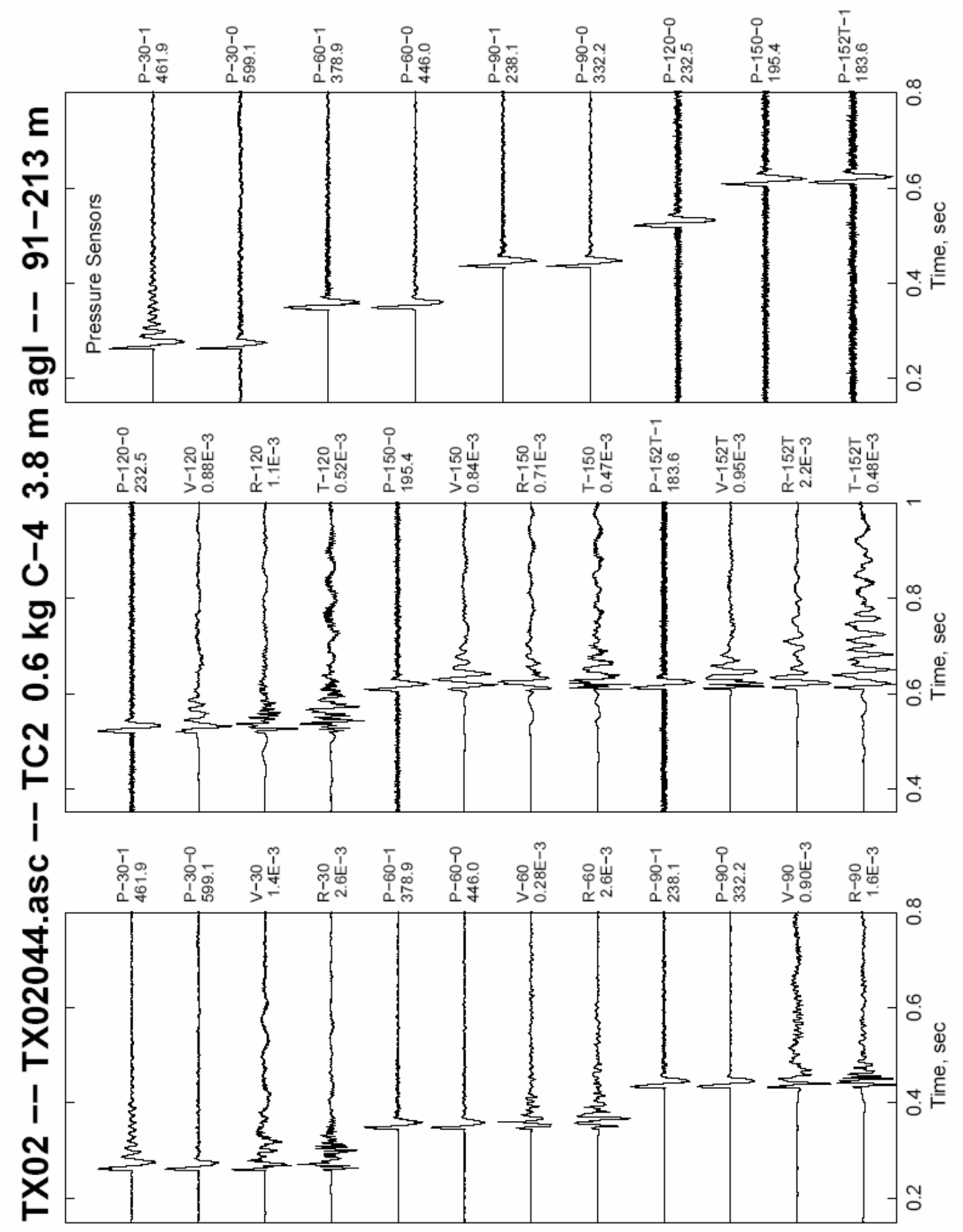




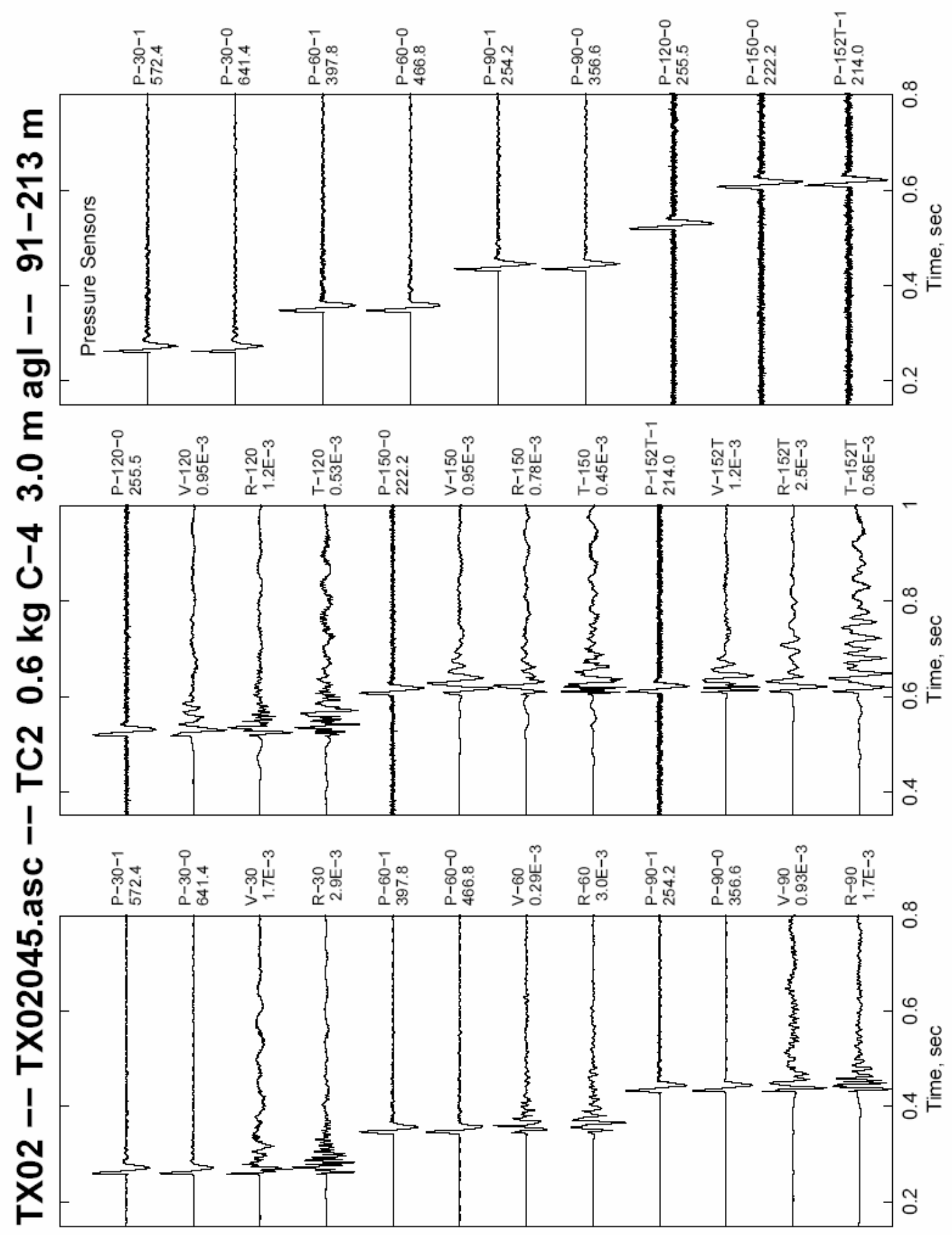




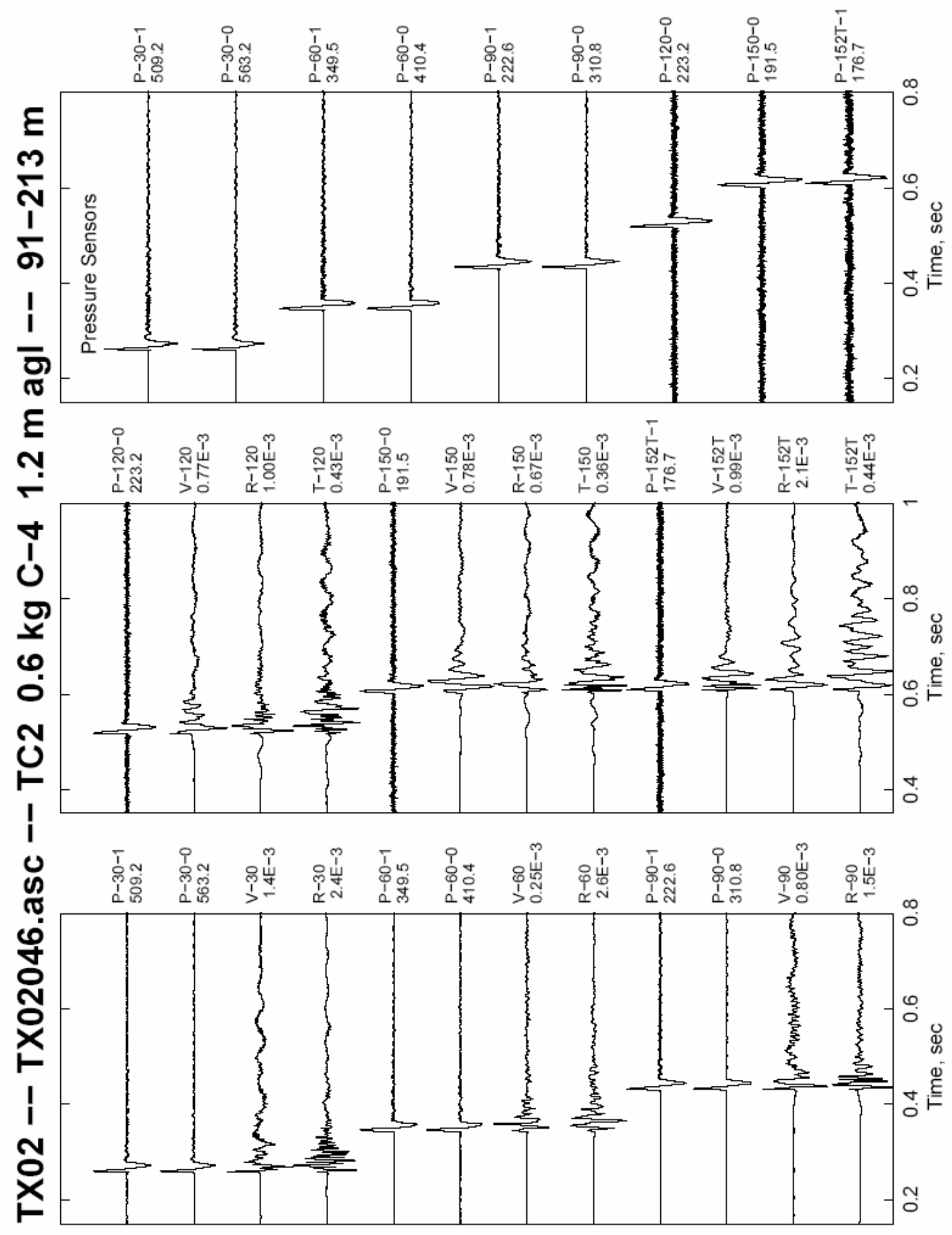




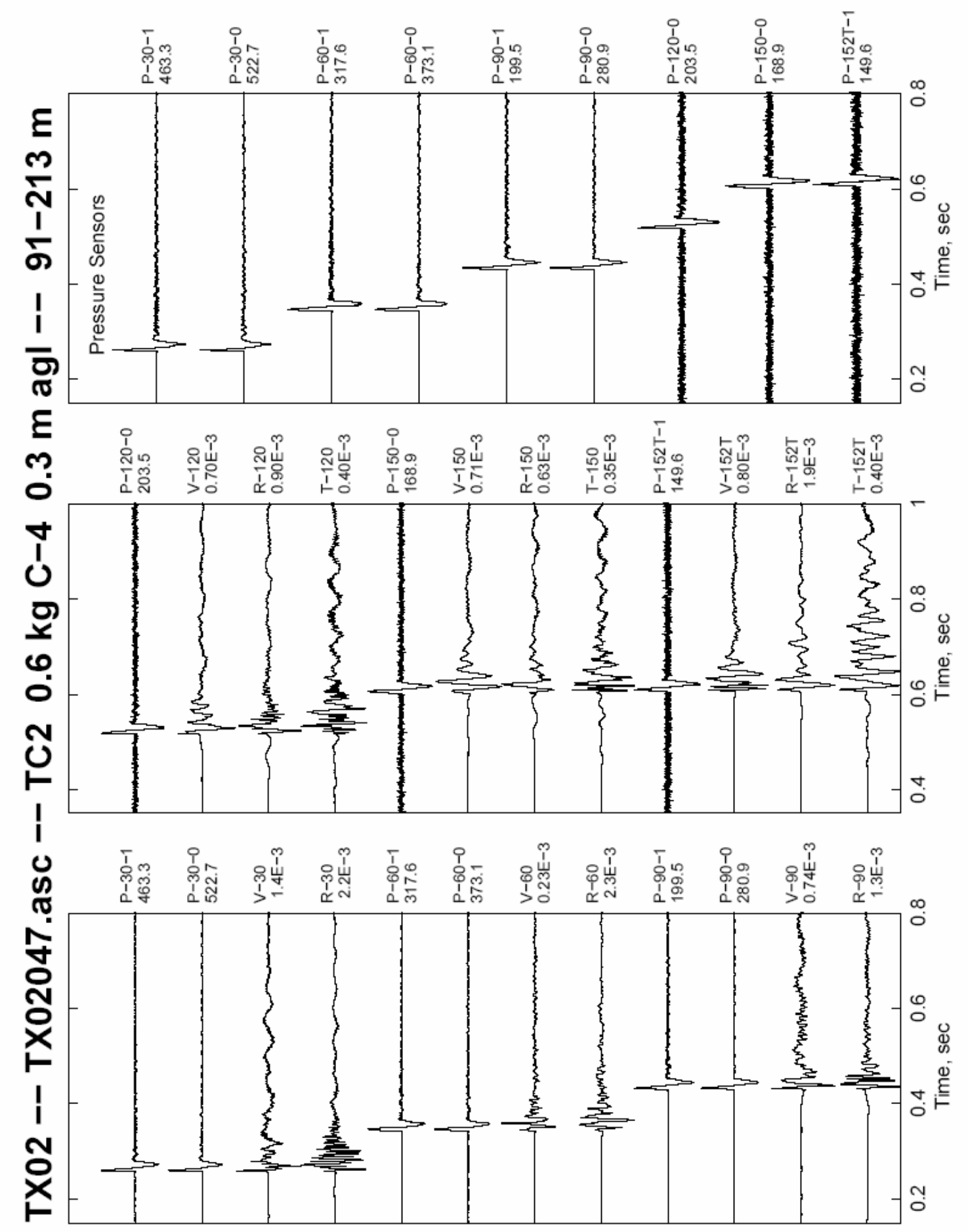




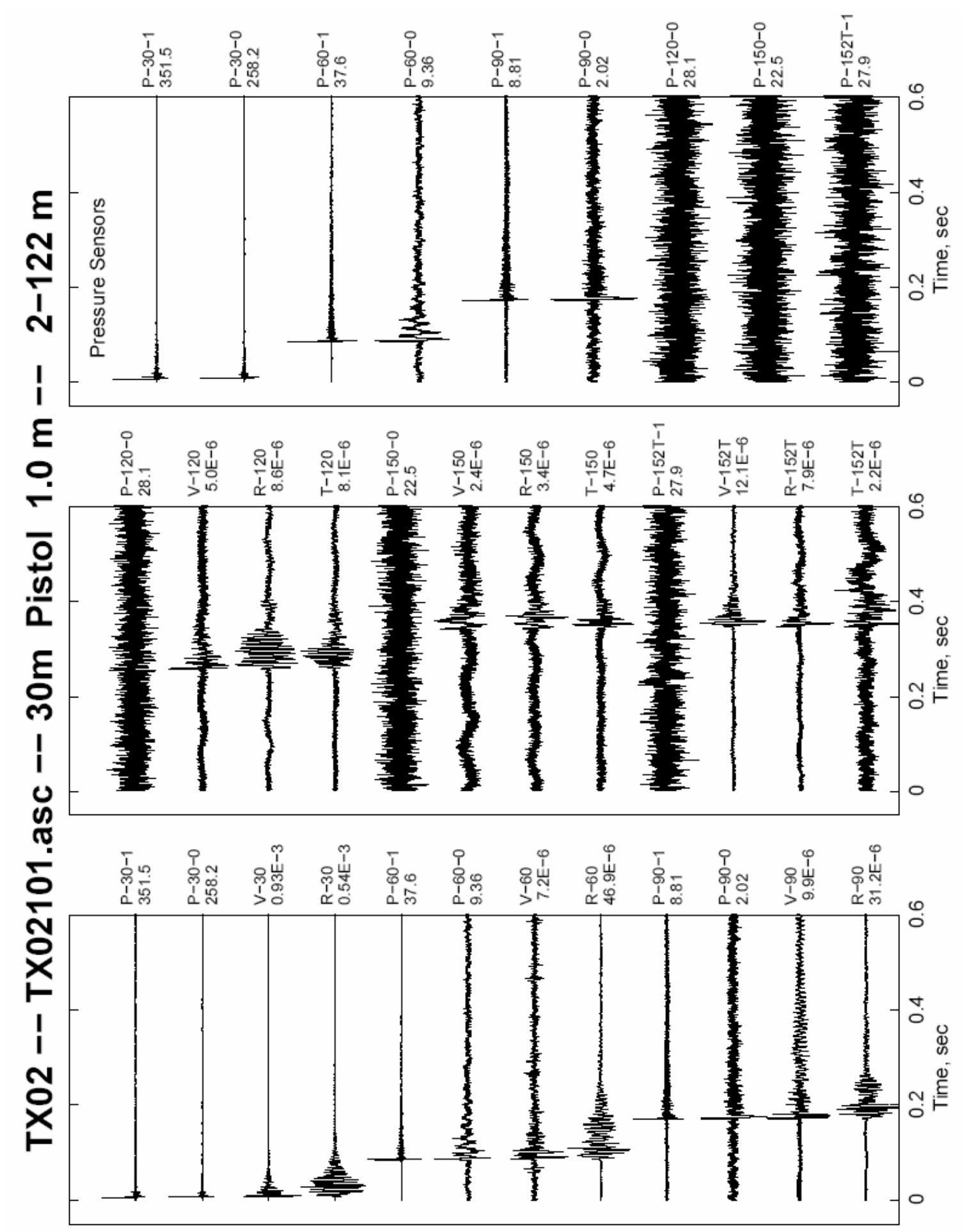




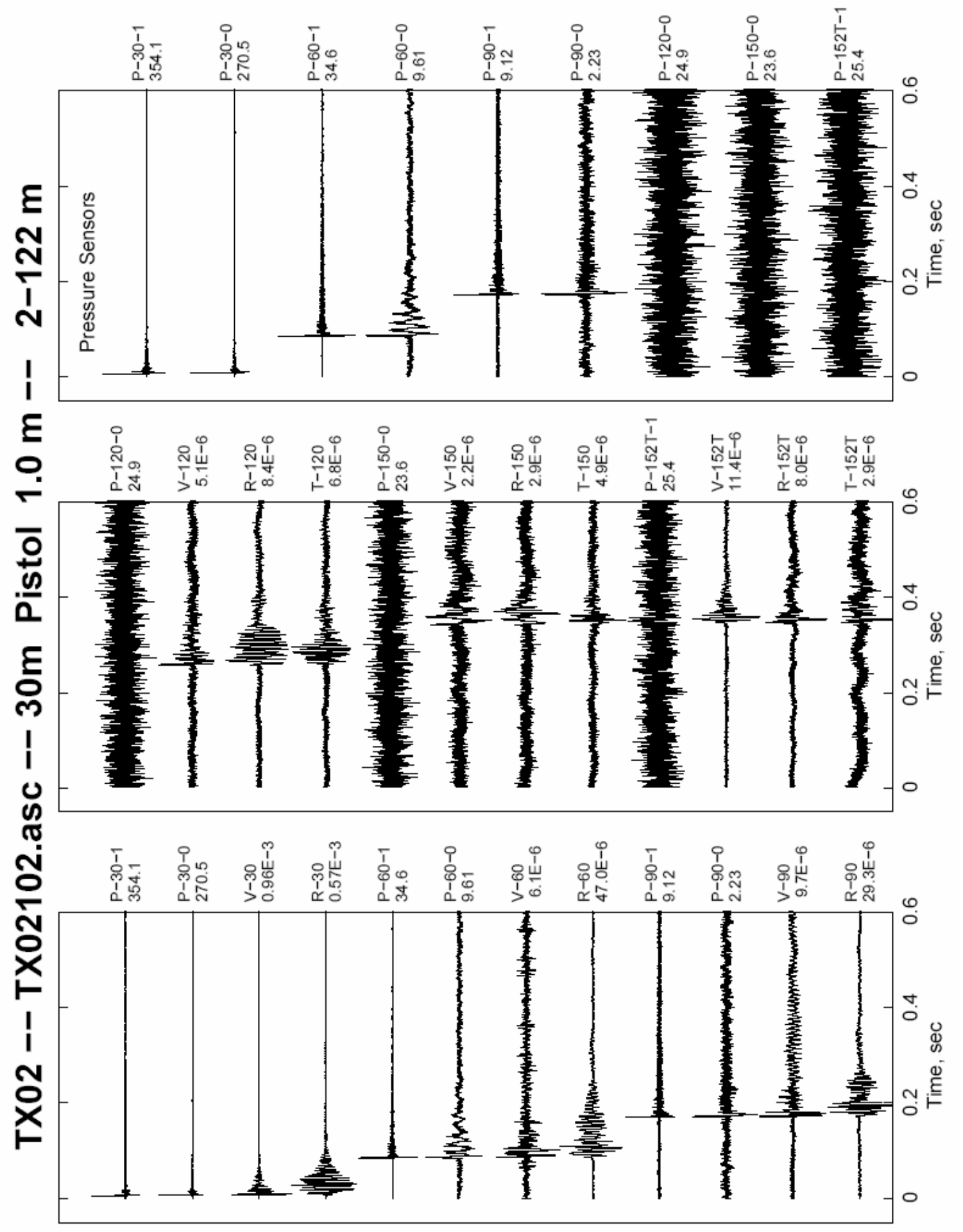




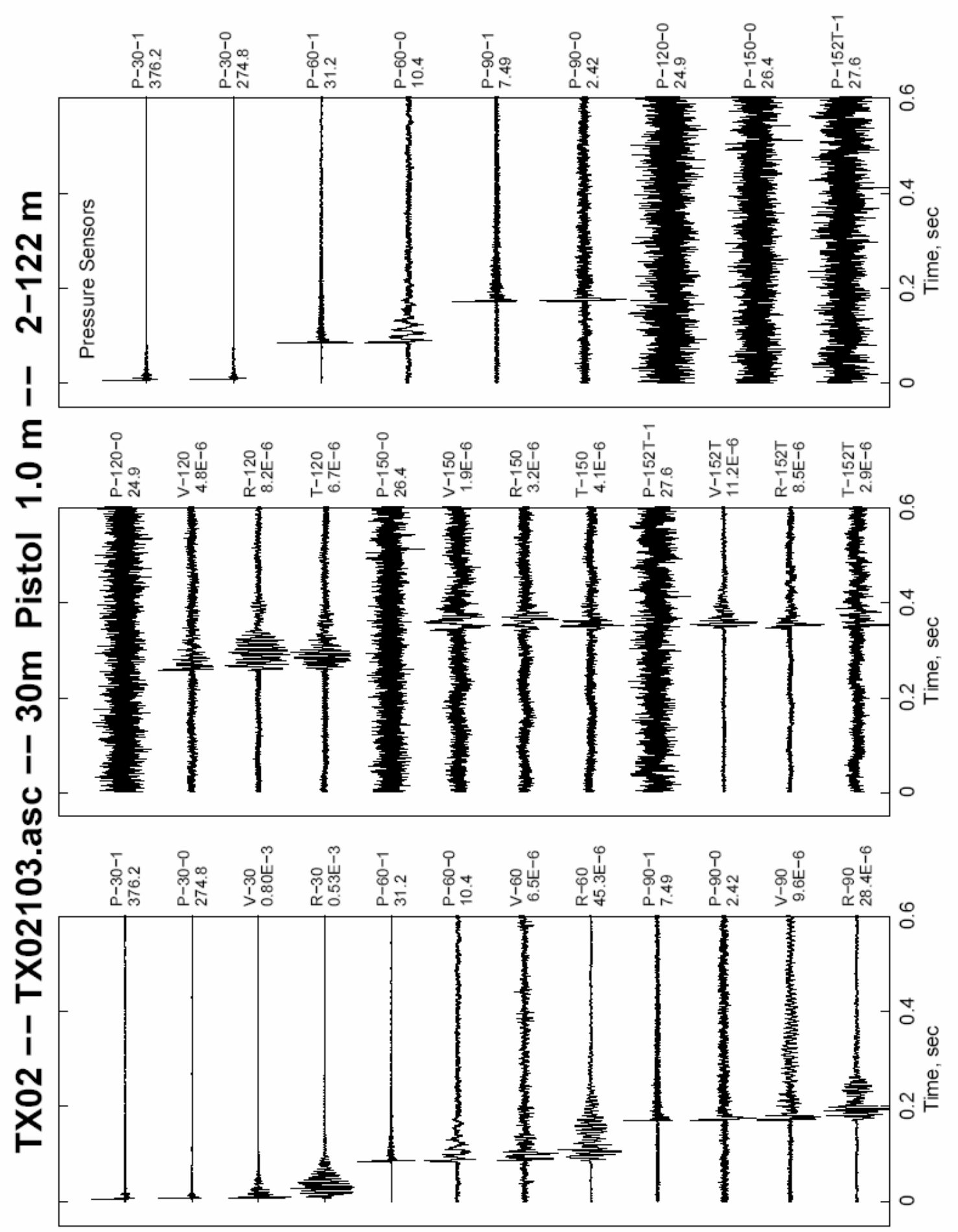




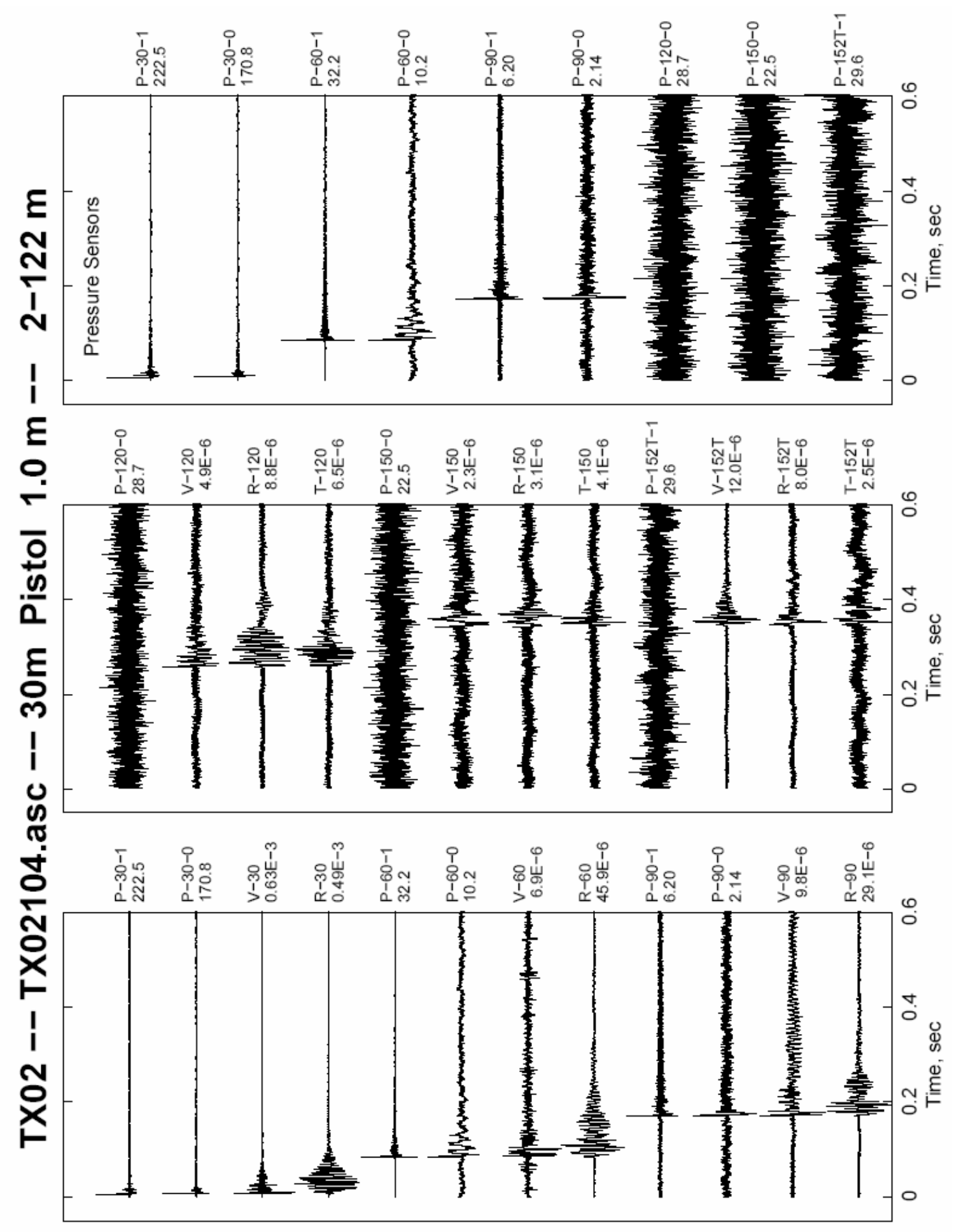




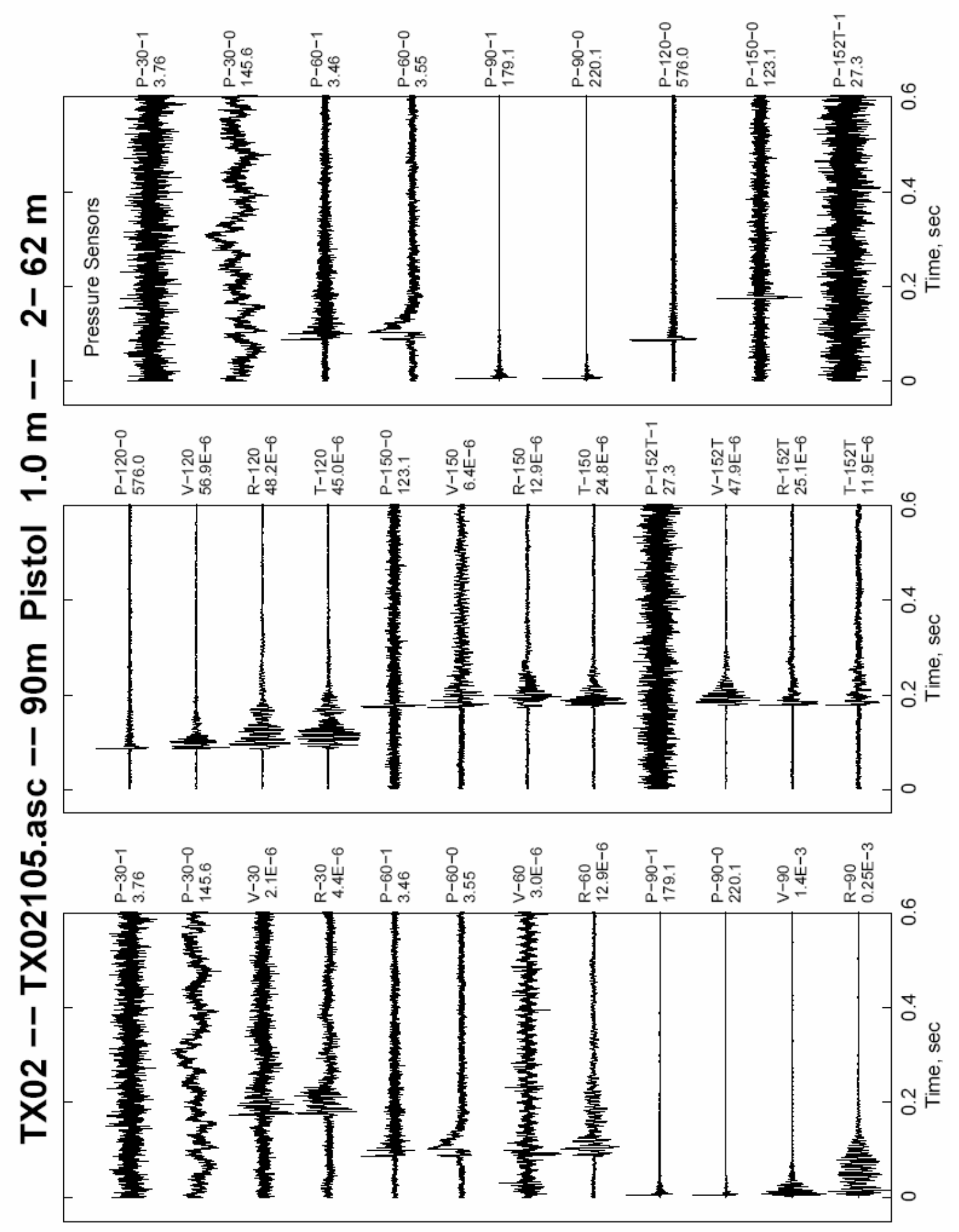




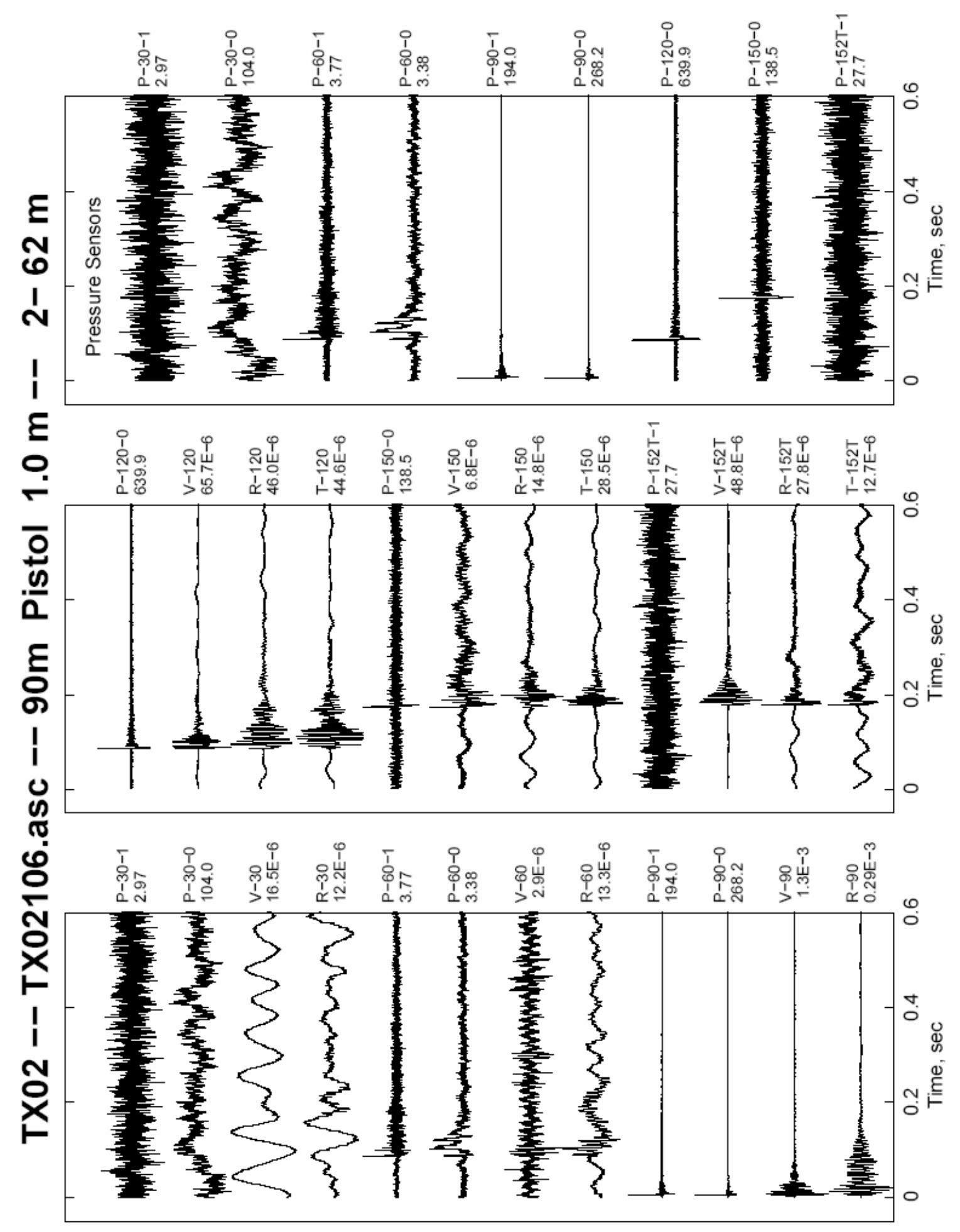




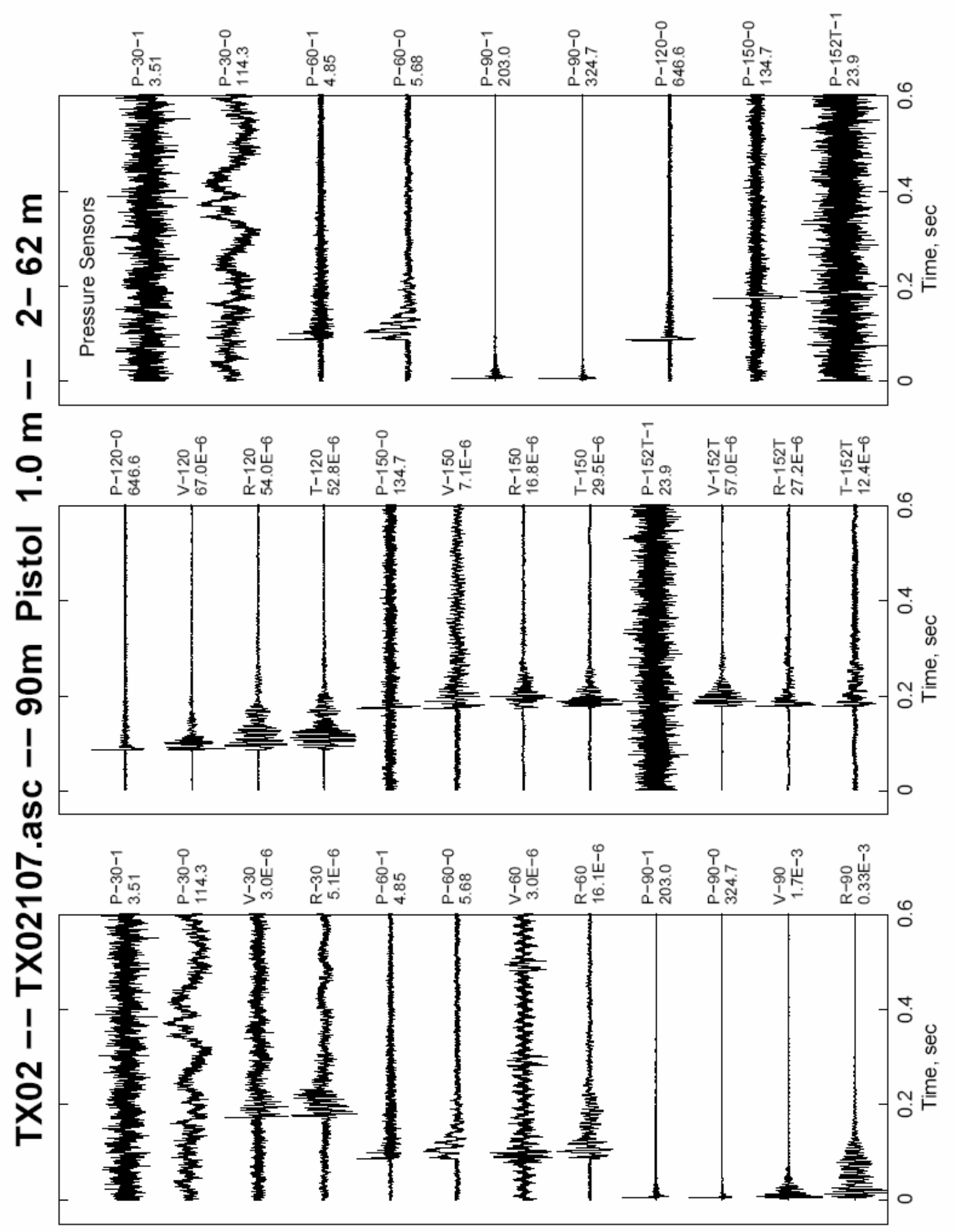




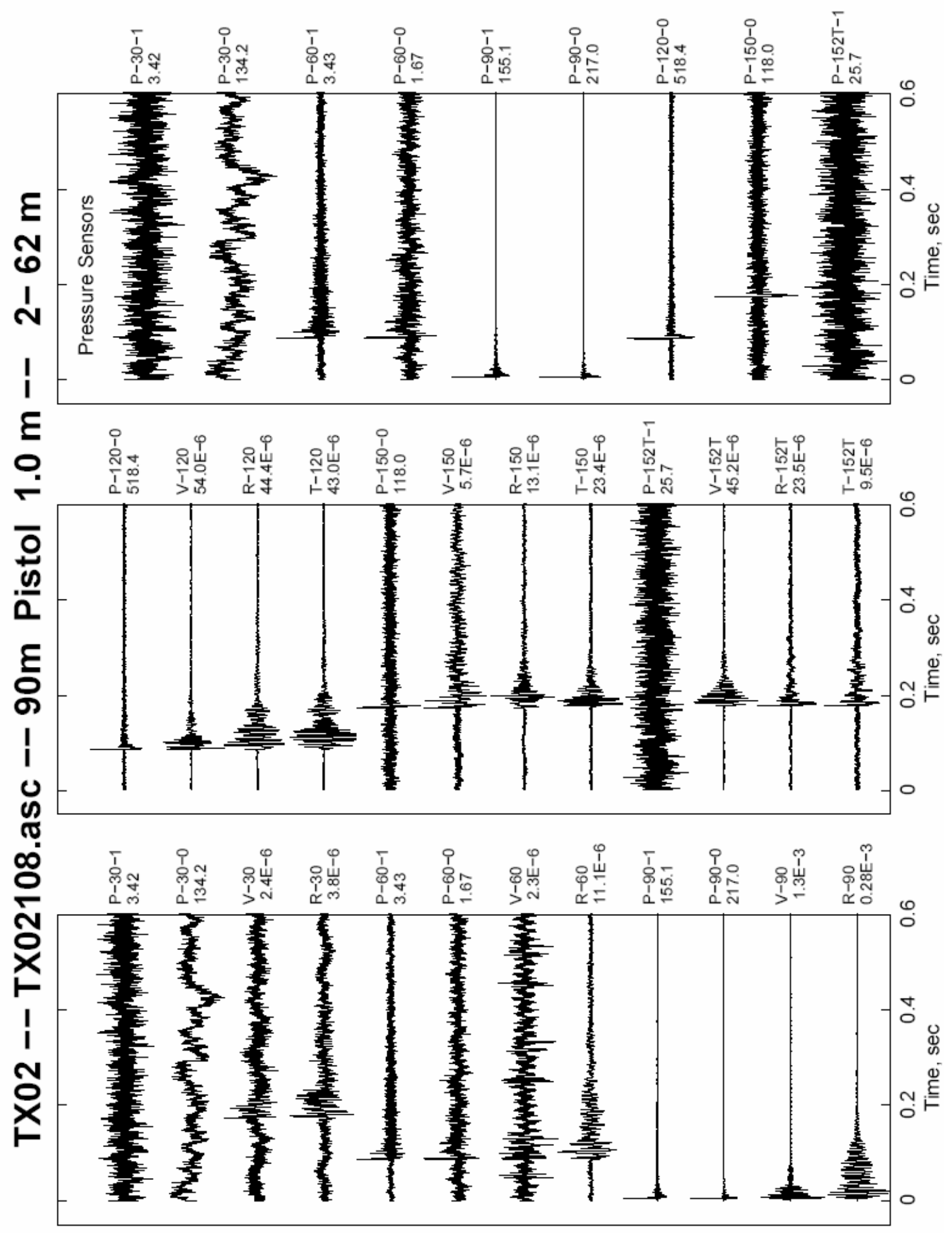




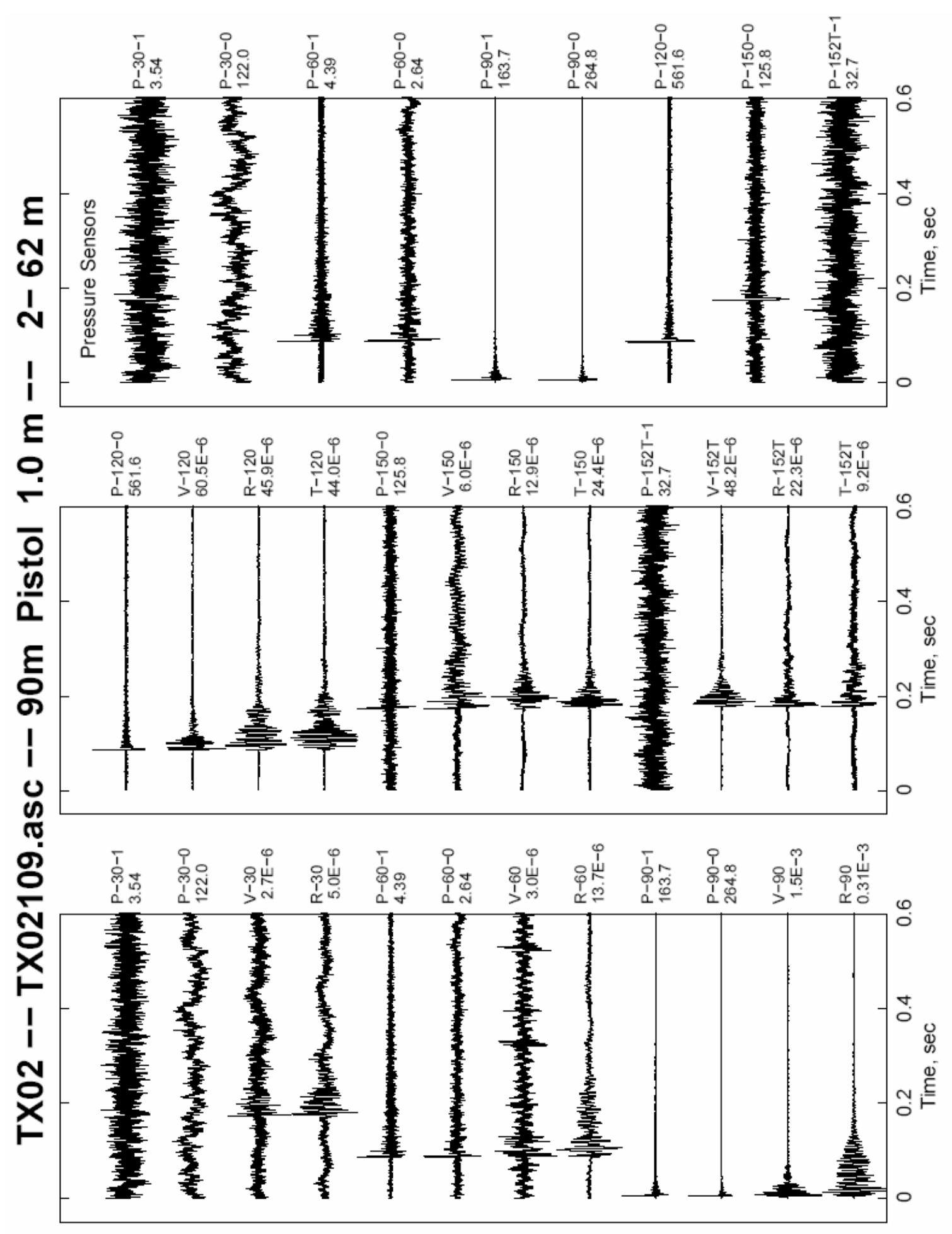




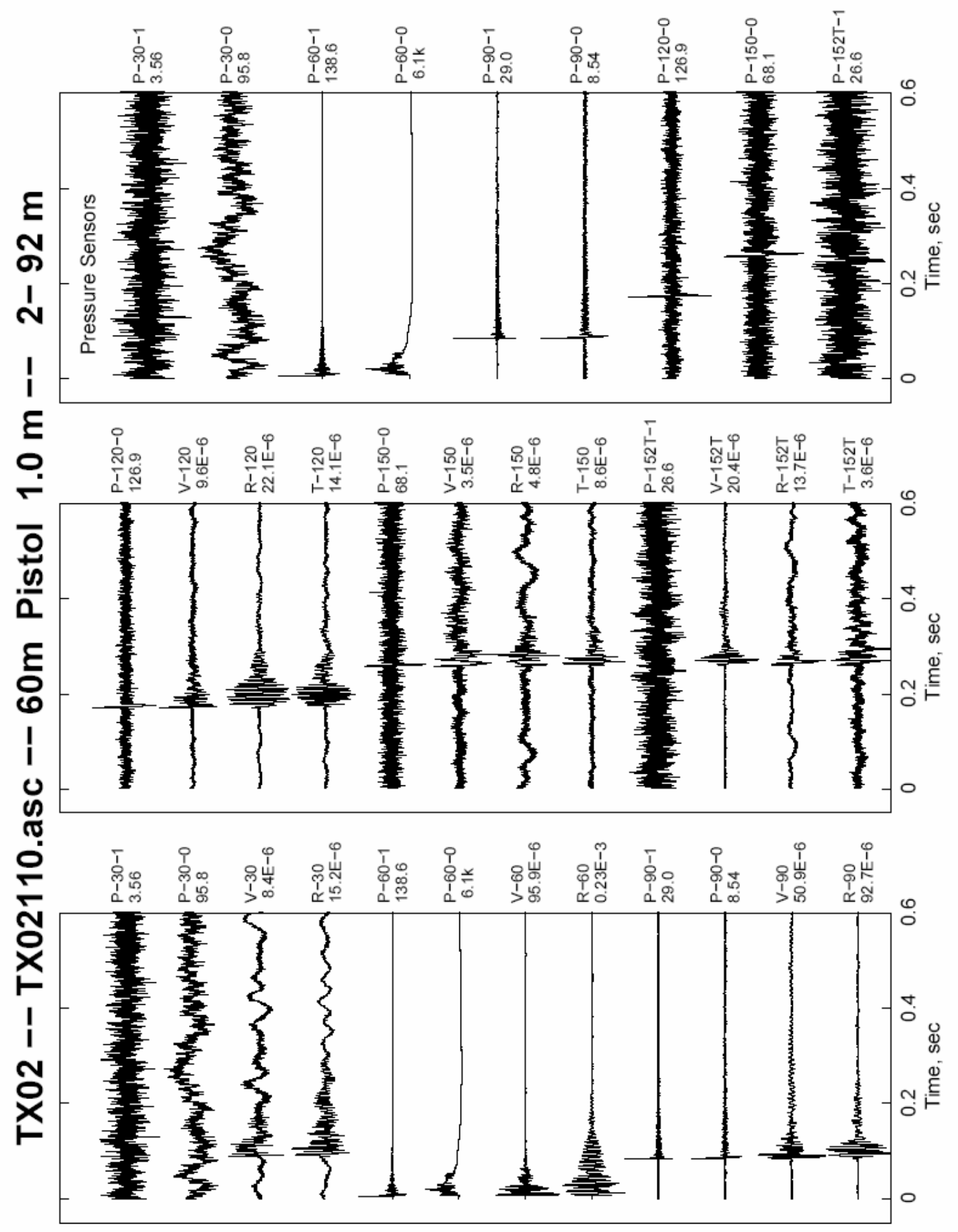




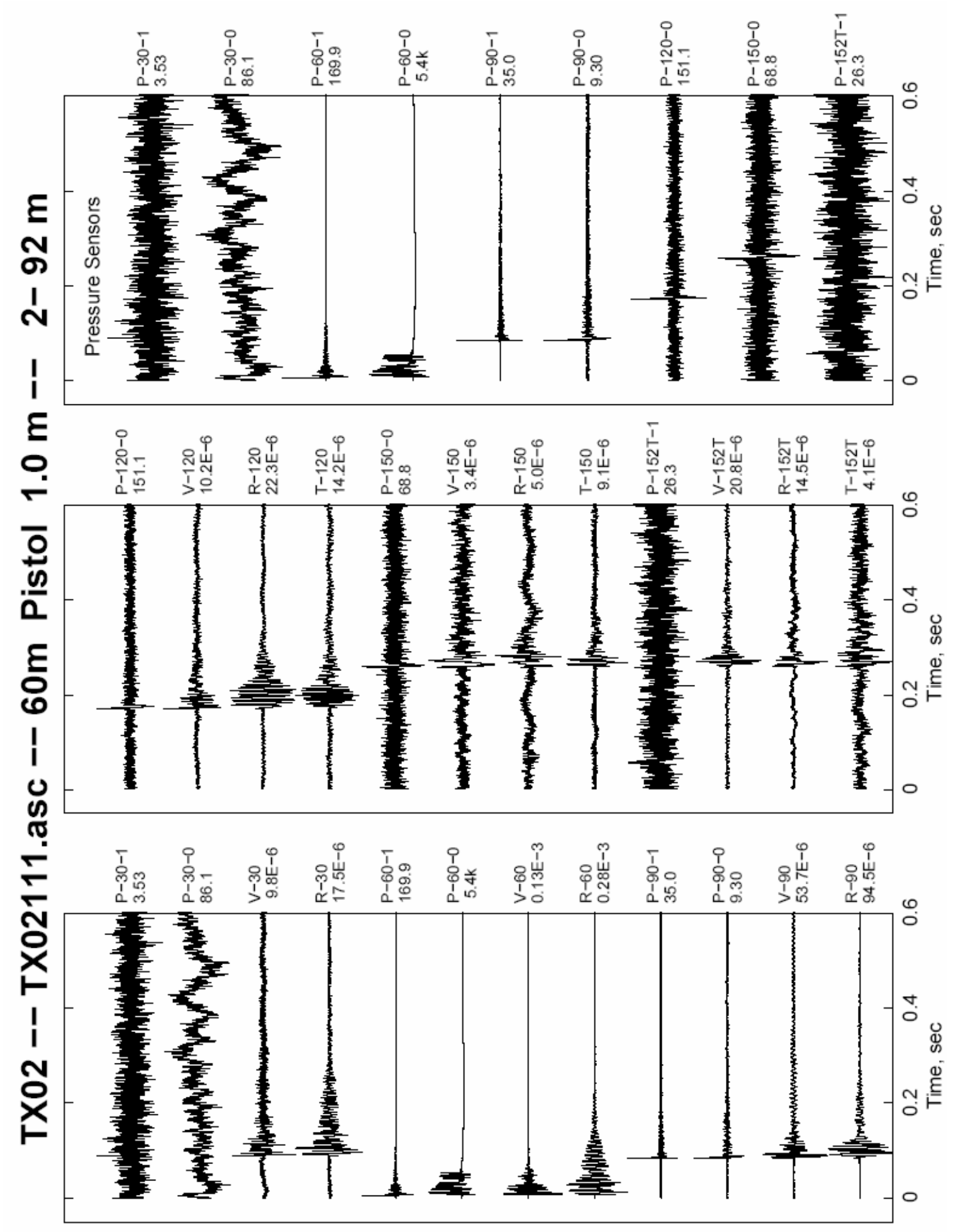




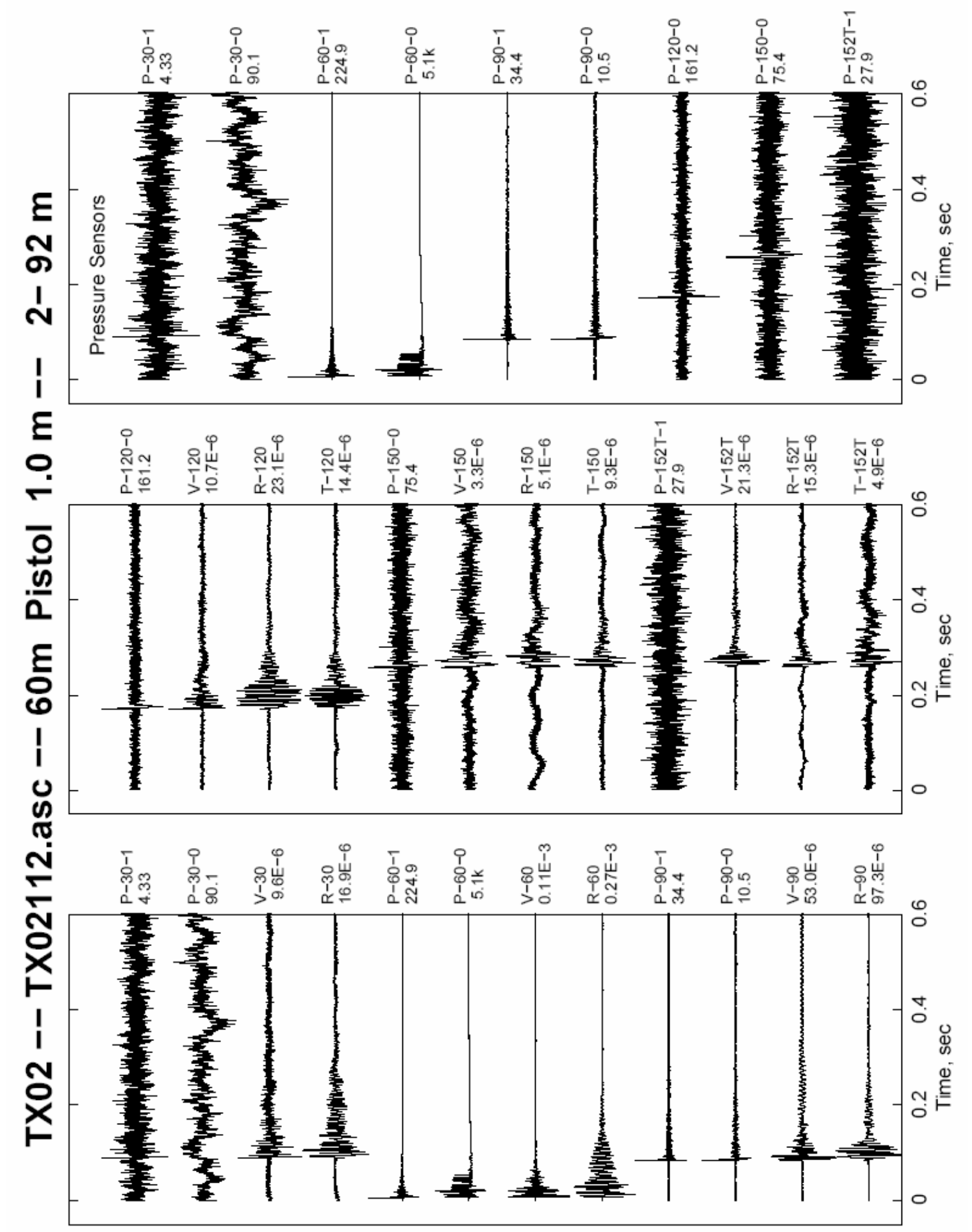




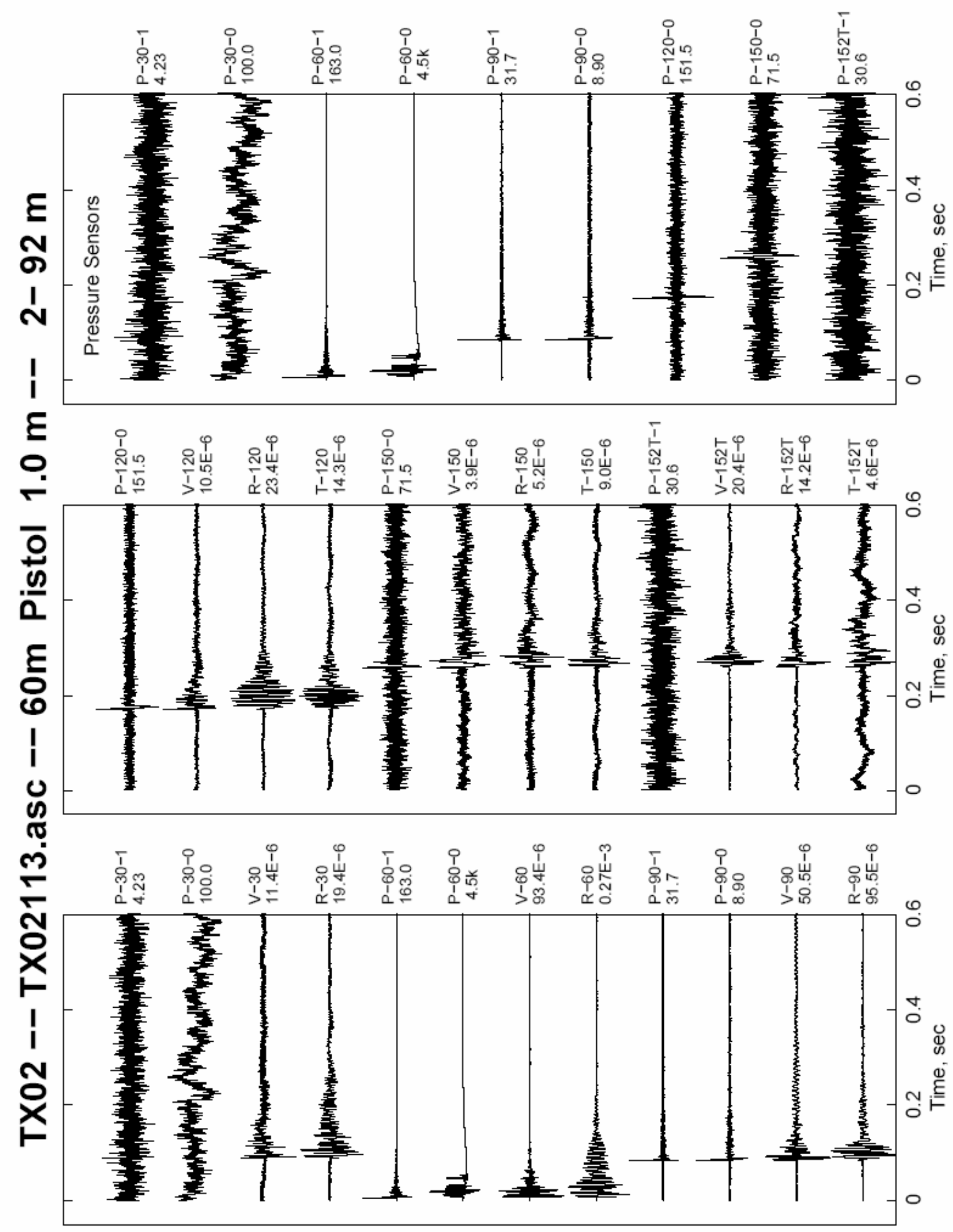




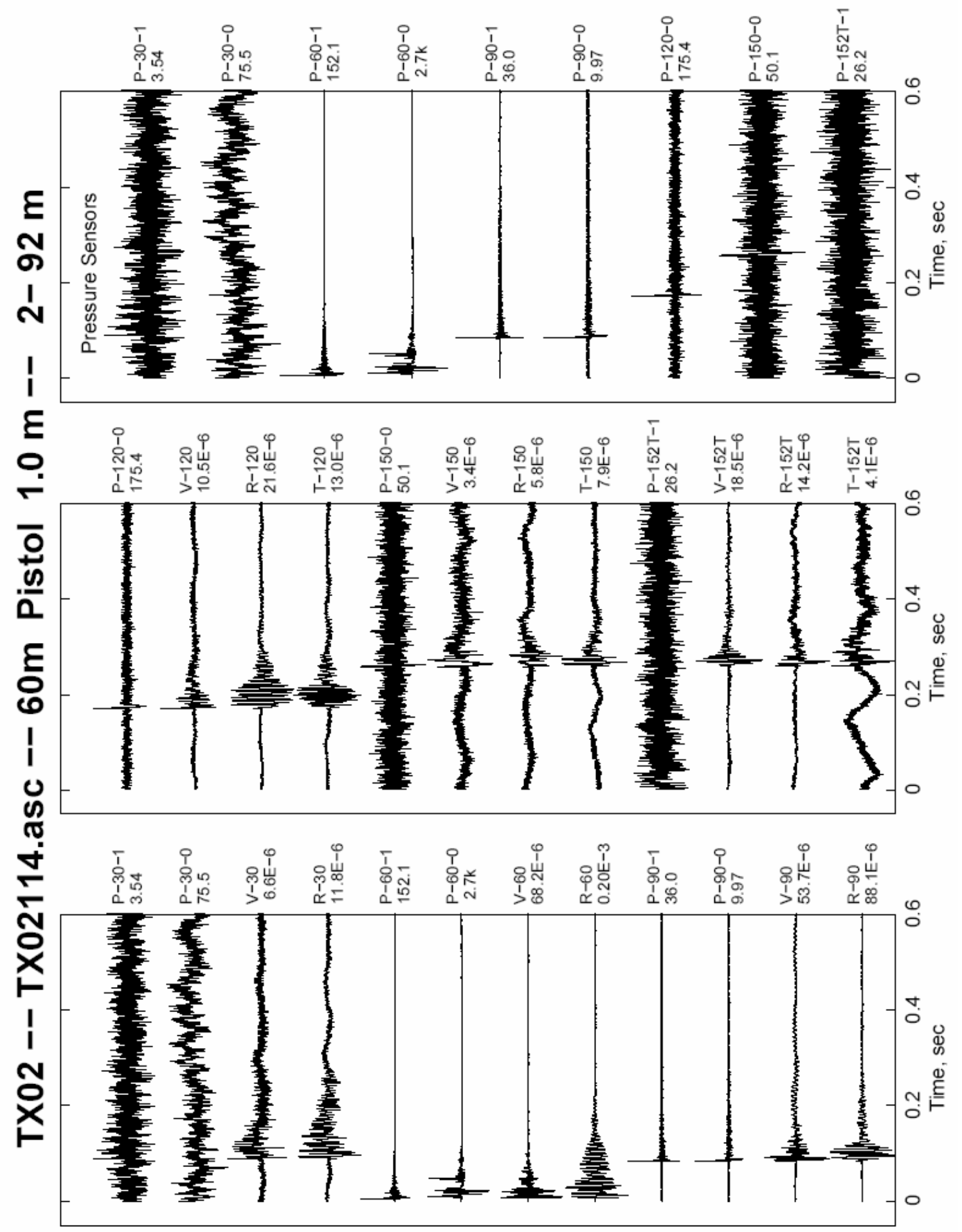




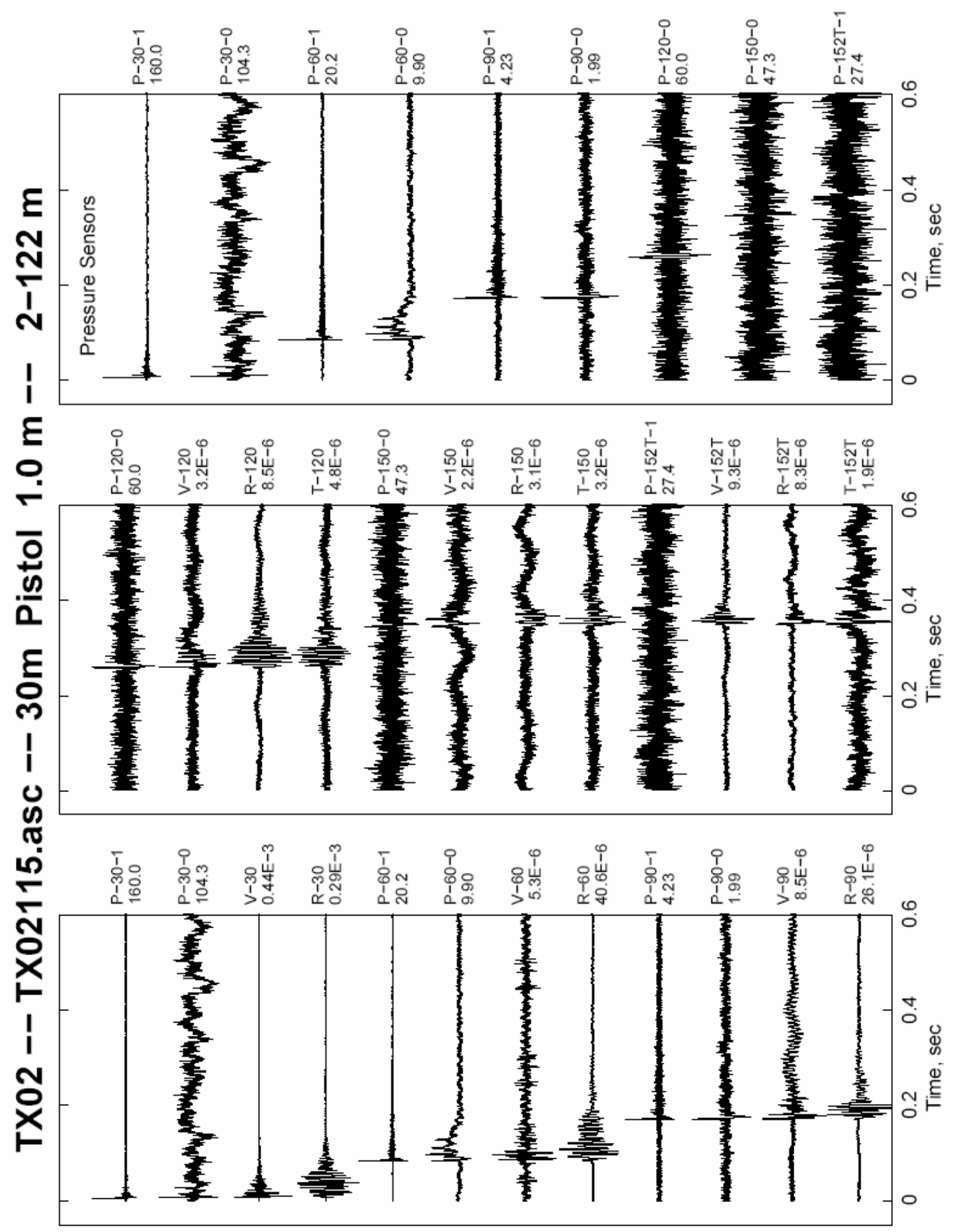




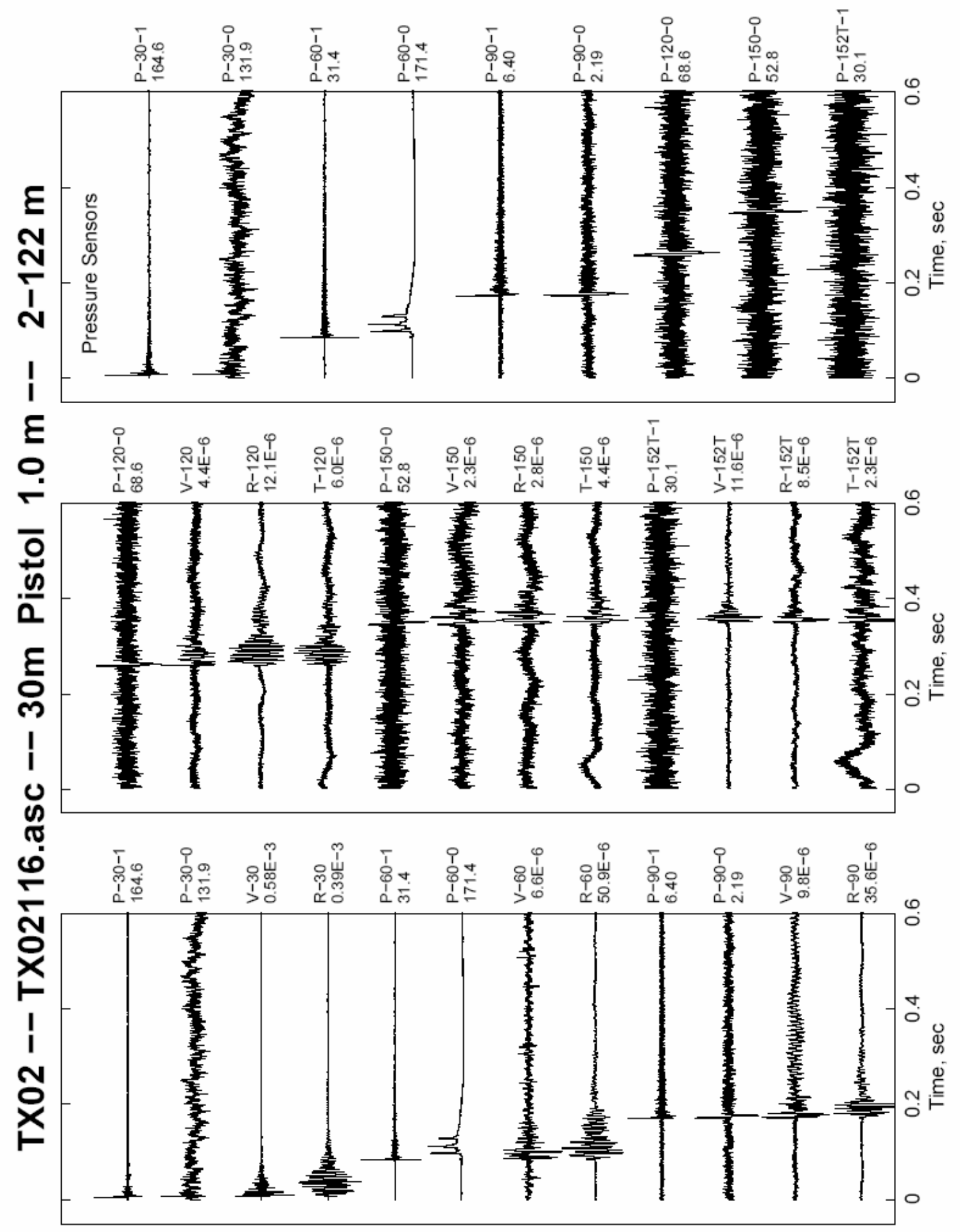




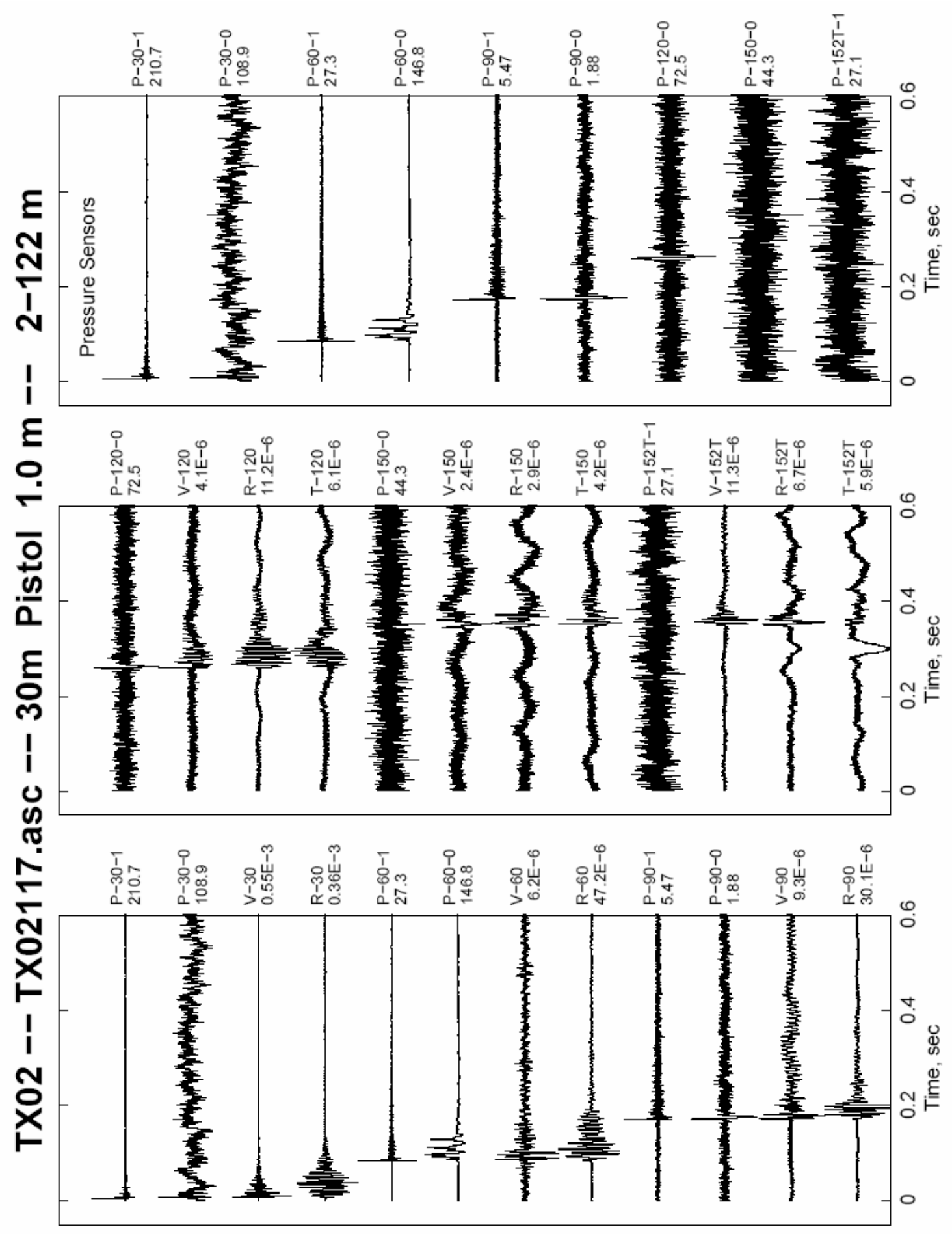




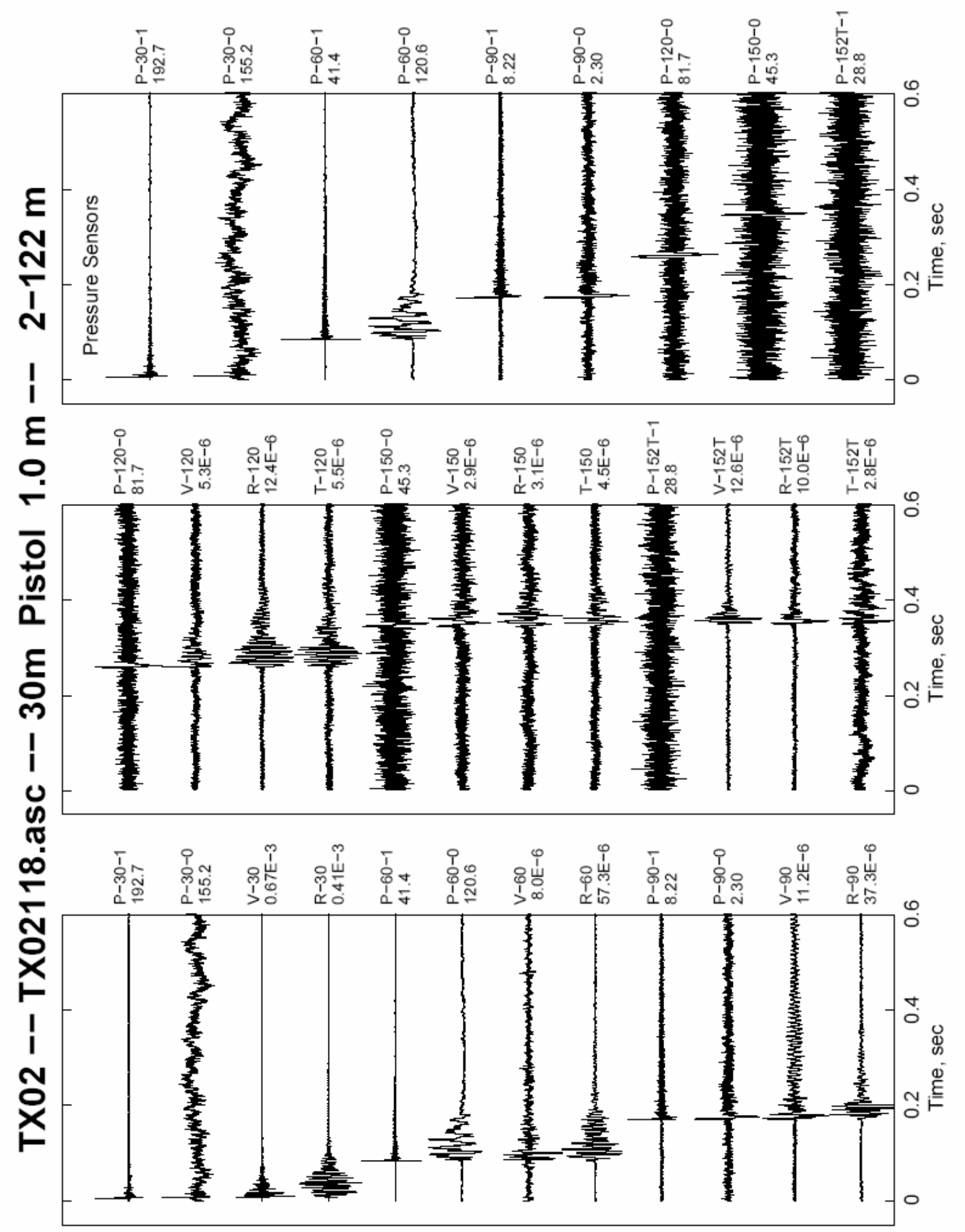




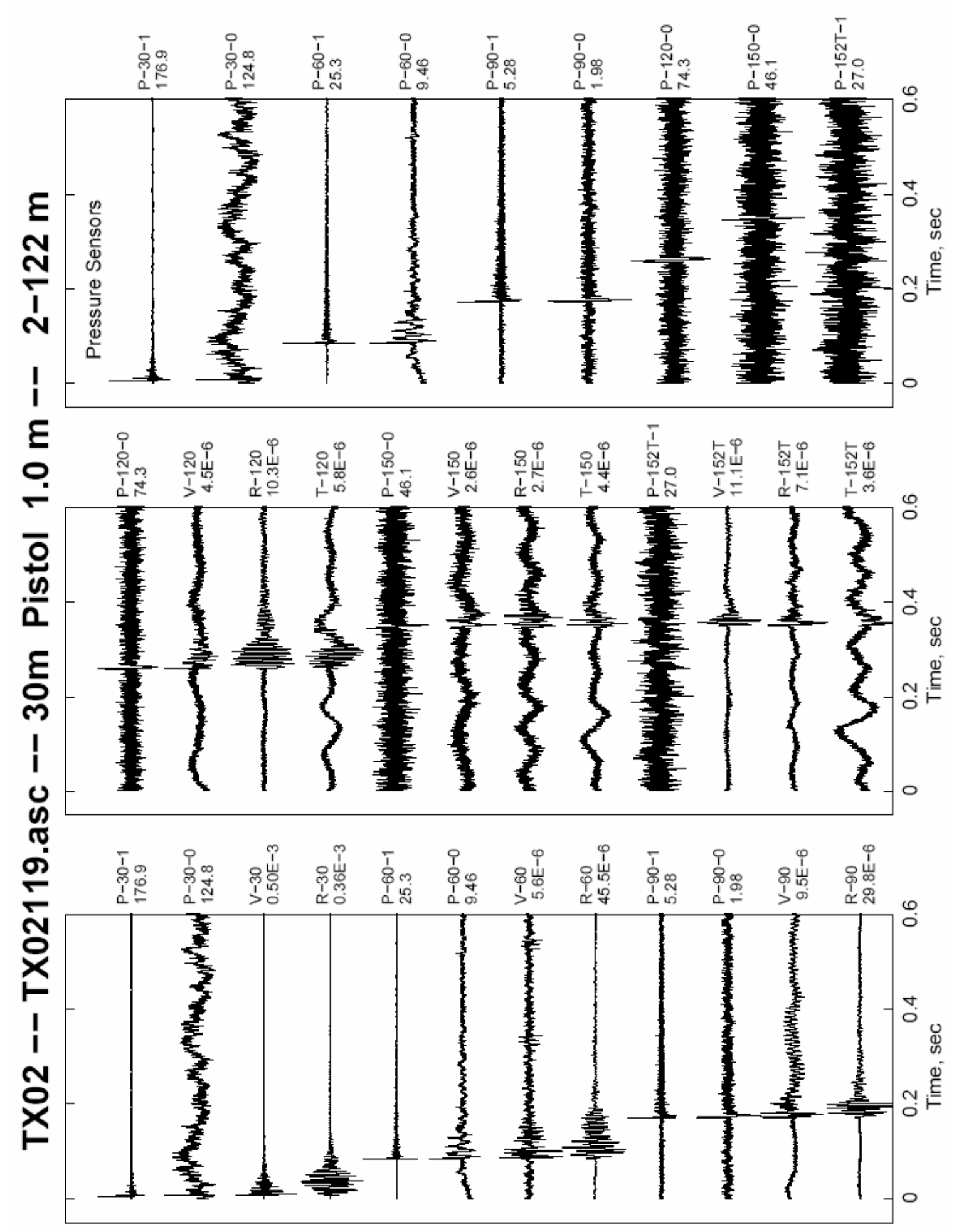




\section{APPENDIX B: MATLAB PROGRAMS TO READ AND PLOT BINARY DATA FROM NZ SEISMOGRAPH AND TO WRITE MULTICHANNEL ASCII DATA FILES.}

This section provides a listing of the MATLAB programs used to read and

plot the 24 binary data files recorded by the Geometrics NZ seismograph in SEG2 format. The programs convert the seismograph voltages to physical units before plotting. The programs also correct errors in the shot time (due to a malfunction of the shot box) and write the corrected data to multichannel ASCII data files that can be plotted using the programs listed in the next section of this report. These ASCII files serve as the permanent archive format for the test data. The files that are listed in this section are:

1. tx02doprocessnz.m-Main program to read binary data files and write multichannel ASCII files. To use, set the desired binary file numbers in the variable "recs" and run the program. You may have to change the lines that construct the filenames to use the correct directory for your computer, and you may want to change the print options at the very end of this program to match your printer or delete the plot from the screen after plotting. The program will write an ASCII file with a new record number that either matches the CERL shot number (for C4 explosions) or a new pistol shot number between 101 and 119. Writes the pressure sensor info to text file TX02MikeLog.m. Calls the programs listed below.

2. readnz.m-Function to read the SEG-2 binary file produced by the NZ seismograph. Calls words.m. Writes info to the text file readnzlog.m.

3. tx02cerlnum.m-Function to select the CERL C4 shot number or new pistol shot number from the binary file number.

4. tx02label.m-Function to get all of the experimental details including which sensor was attached to which channel, voltage-to-physical-units conversion factors, sensor and shot point geometry and distances, which source used for each file, etc. Calls words.m.

5. tx02plotnz.m-Function to make a three-panel plot of the binary data in landscape orientation. The channels plotted in each of the panels are given by the contents of iplot1, iplot2, and iplot3. These plots show the uncorrected times in the binary files, i.e., when the recorder button was pushed, not when the shot was actually fired.

6. tx02tbcorr.m-Function to find the true time break (shot instant). This is done using the arrival times from the first and last sensor in the array to determine the acoustic wave velocity, and then projecting back to zero 
distance to get the origin time. Writes the time break info to file Tx02TimeBreakLog.m and applies the correction to the multichannel ASCII data files.

7. tx02writenchan.m-Function to write multichannel ASCII data file with 12 line trace headers. The ASCII file name is tx02nnn.asc, where nnn is a new file number. nnn = CERL shot number if it was a C4 shot.

8. words.m-Function to separate a string into individual words by finding the blank characters.

\section{tx02doprocessnz.m-Main program to plot binary data and write multichannel ASCII file}

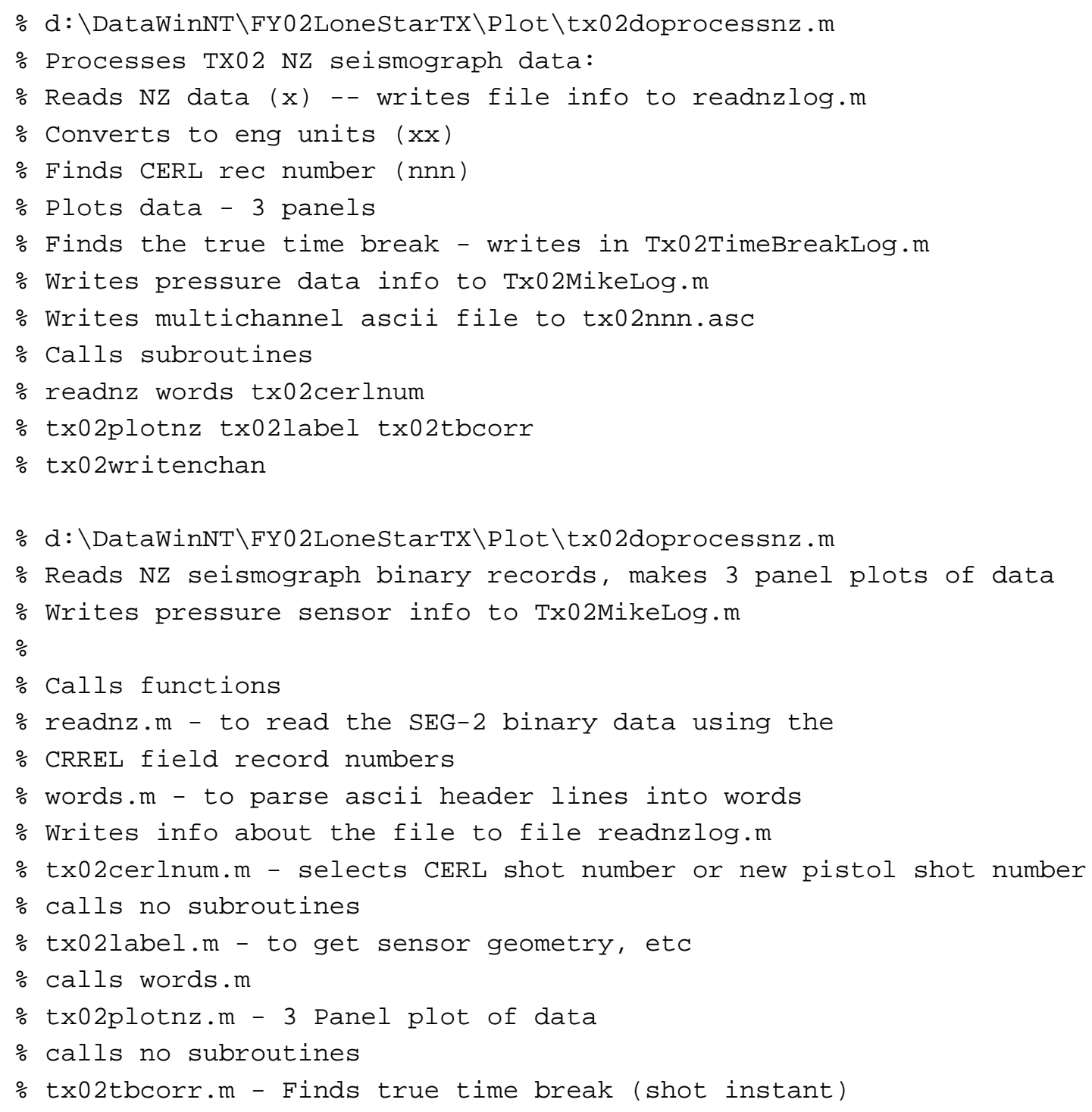


$\%$ from the arrival times at a few of the sensors

$\%$ Writes info to TX02TimeBreakLog.m

$\%$ calls no subroutines

$\%$ tx@2writenchan.m - writes multichannel ascii data with 12 line trace headers

\% File name is tx@2nnn.asc, where nnn is a new file number

$\%$ nnn $=$ CERL Shot number if it was a C-4 shot

$\%$ calls no subroutines

\% Don Albert dalbert@crrel.usace.army.mil

\% USA ERDC-CRREL, 72 Lyme Road, Hanover, NH 03755

\% NZ binary record numbers; plot has new record number in title

$\%$ C-4 Records 39, 44 were bad and are omitted

\%recs $=\left[\begin{array}{lllll}65 & 66 & 67 & 68 & 5\end{array}\right] ; \%$ \%X02 $\mathrm{C}-4$ examples and blank pistol shot

example

$\%$ recs $=\left[\begin{array}{llll}3 & 5 & 10 & 15\end{array}\right] ;$ \%Blank pistol shot examples

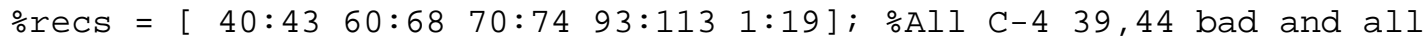

blank pistol

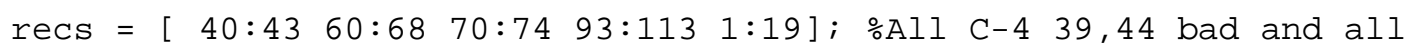

blank pistol

recs $=\left[\begin{array}{ll}64 & 65\end{array}\right]$;

\% Loop to plot the files

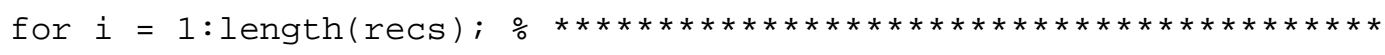

$\mathrm{xx}=[\mathrm{]} ; \mathrm{x}=[\mathrm{]}$;

recnum $=\operatorname{recs}(i)$;

shotnum $=\operatorname{recs}(i)$;

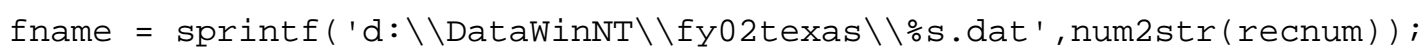

$\%$ read in data from $N Z$ binary file - $x$

$[x$, npts, deltat, nchan, delayms, descalingfact, ...

stackcount, acqdate, acqtime] = readnz (fname);

$\%$ sample rate

srate $=1 /$ deltat $; \%$ number of samples per second

$\%$ Get geometry and description for this experiment and record number

[sensor, sensortype, rtype, sensorx, sensory, sensorz, ...

srcloc, sourcetype, stype, srcx, srcy, srcz, sourcesize, ...

engfact, dist, sensor 2 , source 2$]=t x 02$ label $(x$, shotnum, nchan $)$; 
\% Convert from voltage to physical units ( $\mathrm{Pa}$ or $\mathrm{m} / \mathrm{s}$ )

xgain = descalingfact./(stackcount. ${ }^{*}$ engfact $) ;$ \%recorder units to eng units

for $i i i=1$ :nchan; $x x(:, i i i)=x(:, i i i) * x g a i n(i i i) ;$ end

$x x \max =\max (\operatorname{abs}(x x))$; \%abs max for plotting

$\%$ get CERL record number for $\mathrm{C}-4$ shots

cerlrec $=0$;

[cerlrec ] = tx@2cerlnum (shotnum);\%If C-4 get CERL Shot Number for Plot

$\% 3$ panel plot of $\mathrm{nz}$ data

iplot1 = [ $\left.\begin{array}{llllllllllll}9 & 10 & 11 & 12 & 5 & 6 & 7 & 8 & 1 & 2 & 3 & 4\end{array}\right]$; \%Channels for 1st (left) panel

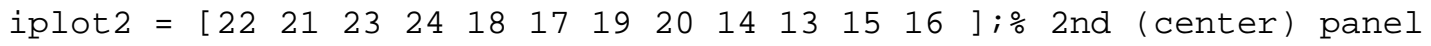

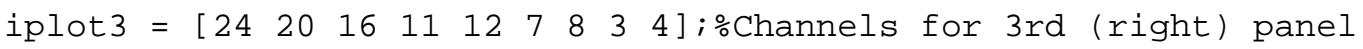

tx@2plotnz (shotnum, cerlrec, xx, iplot1, iplot2, iplot3, deltat, delayms, ...

dist, sensor, sensorx, sensory, sensorz, ...

srcloc, sourcesize, sourcetype, srcx, srcy, srcz);

\% Write pressure sensor data to text file

fid3=fopen ( 'TxO2MikeLog. $\mathrm{m}$ ', ' a' ) ;

iplot $=$ iplot $3 ;$ nplot=length (iplot 3$)$;

$\%$ Write pressure sensor trace header info to logfile

$[a a b b]=\max (x x)$; \%set plot windows because manual TB

$[\mathrm{cc} d d]=\min (x \mathrm{x})$; \%set plot windows because manual $\mathrm{TB}$

for $i$ ii $=1$ :nplot;

tttmax $=\operatorname{delayms} / 1000+(b b($ iplot $(i i i))) /$ srate; \%times in seconds

tttmin $=\operatorname{delayms} / 1000+(\operatorname{dd}($ iplot $(i i i))) /$ srate; \%times in seconds fprintf(fid $3, \ldots$

' \%4.of \%3.of \%3.of \%3.1f \%5.of \%3.1f \%3.1f \%6.1f \%6.1f \%6.4f \%6.1f

$\% 6.4 f \% 6.4 f \backslash n ', \ldots$

shotnum, iplot(iii), sensorx(iplot(iii)), sensorz(iplot(iii)), ...

srcx, srcz, sourcesize*1.25/2.2, ...

dist (iplot(iii)), aa(iplot(iii)), tttmax, cc(iplot(iii)), tttmin, (tttmin-

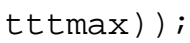

end

fclose (fid3);

\% Find TB correction - writes to output file XxXTimebreaklog.m 


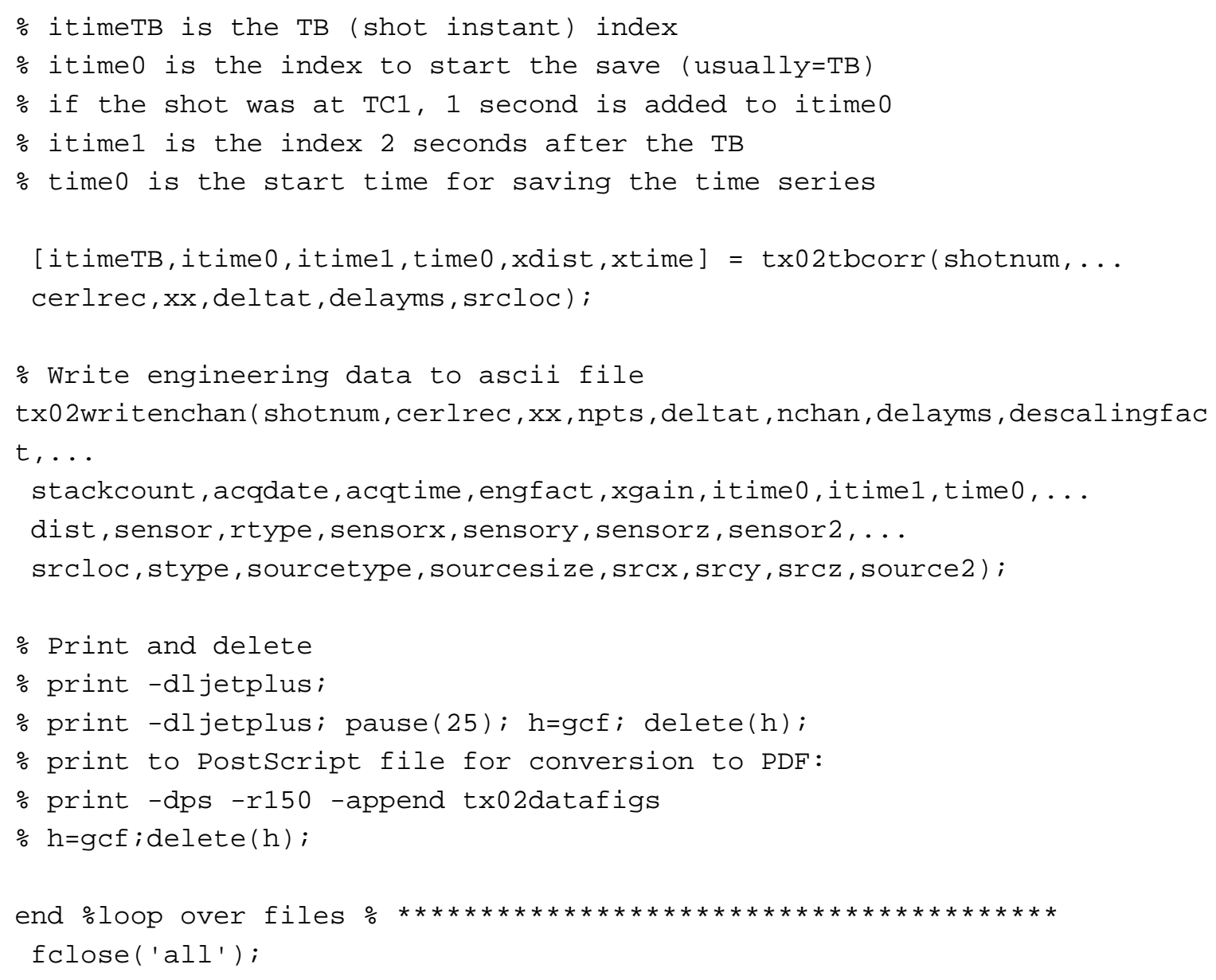




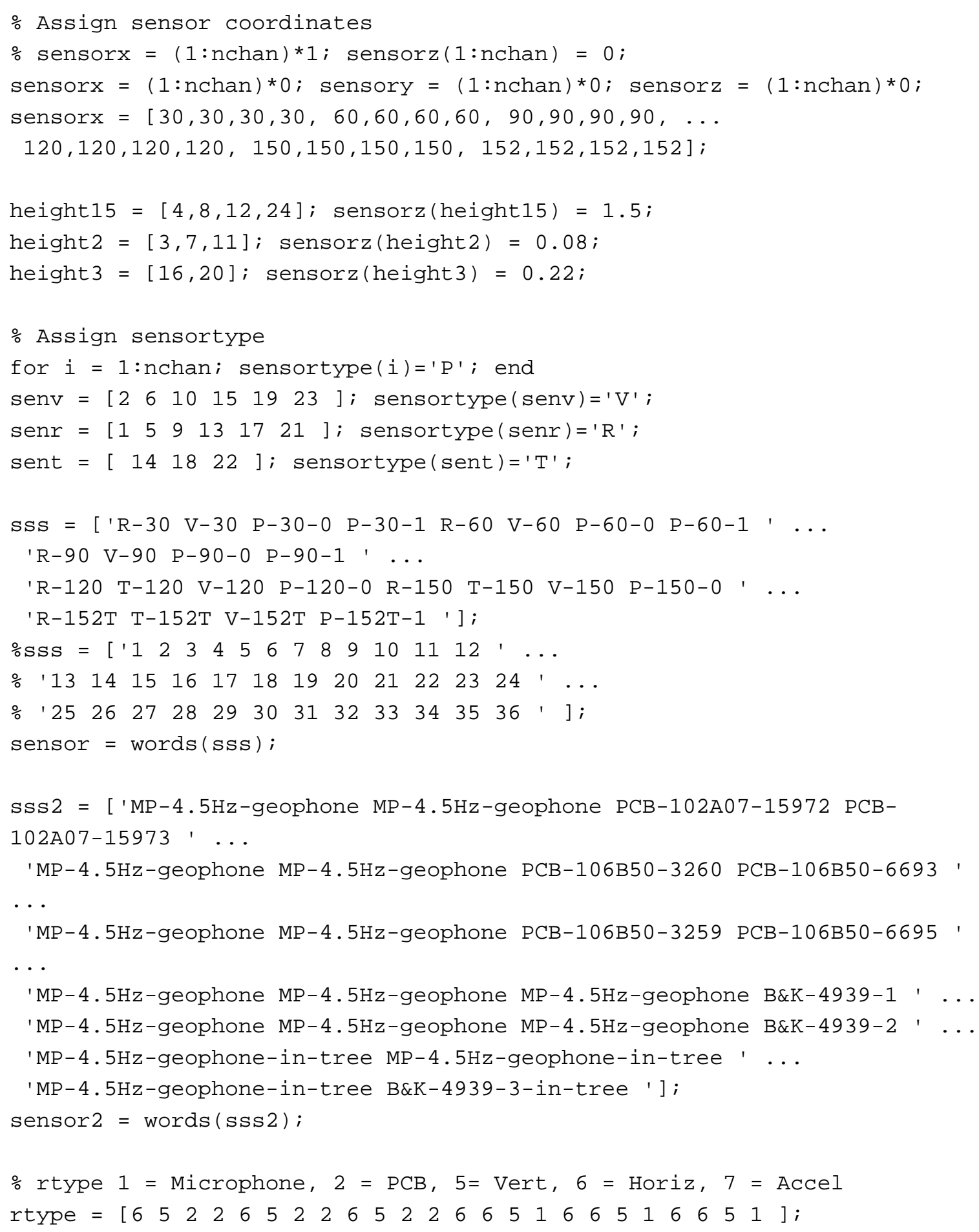




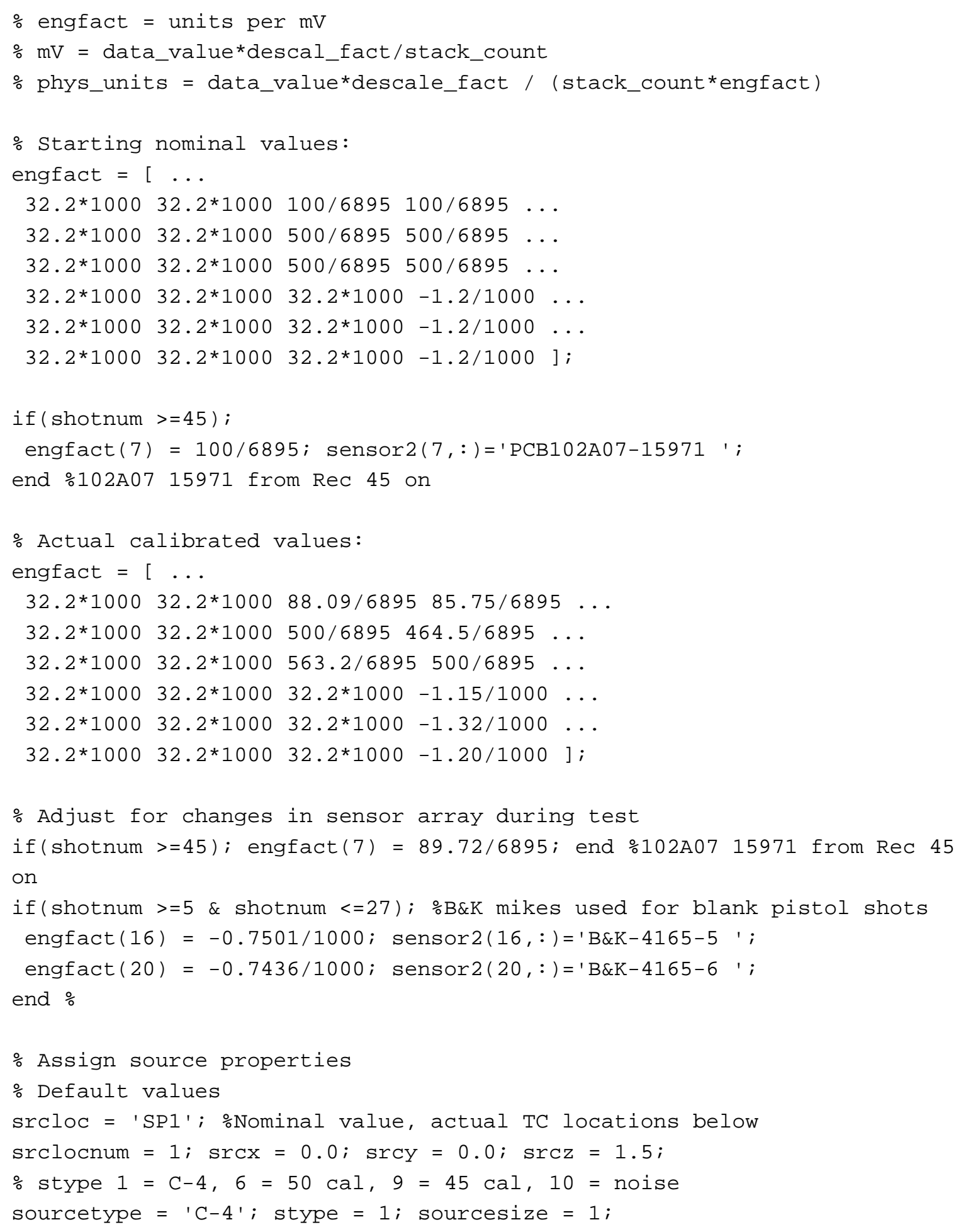




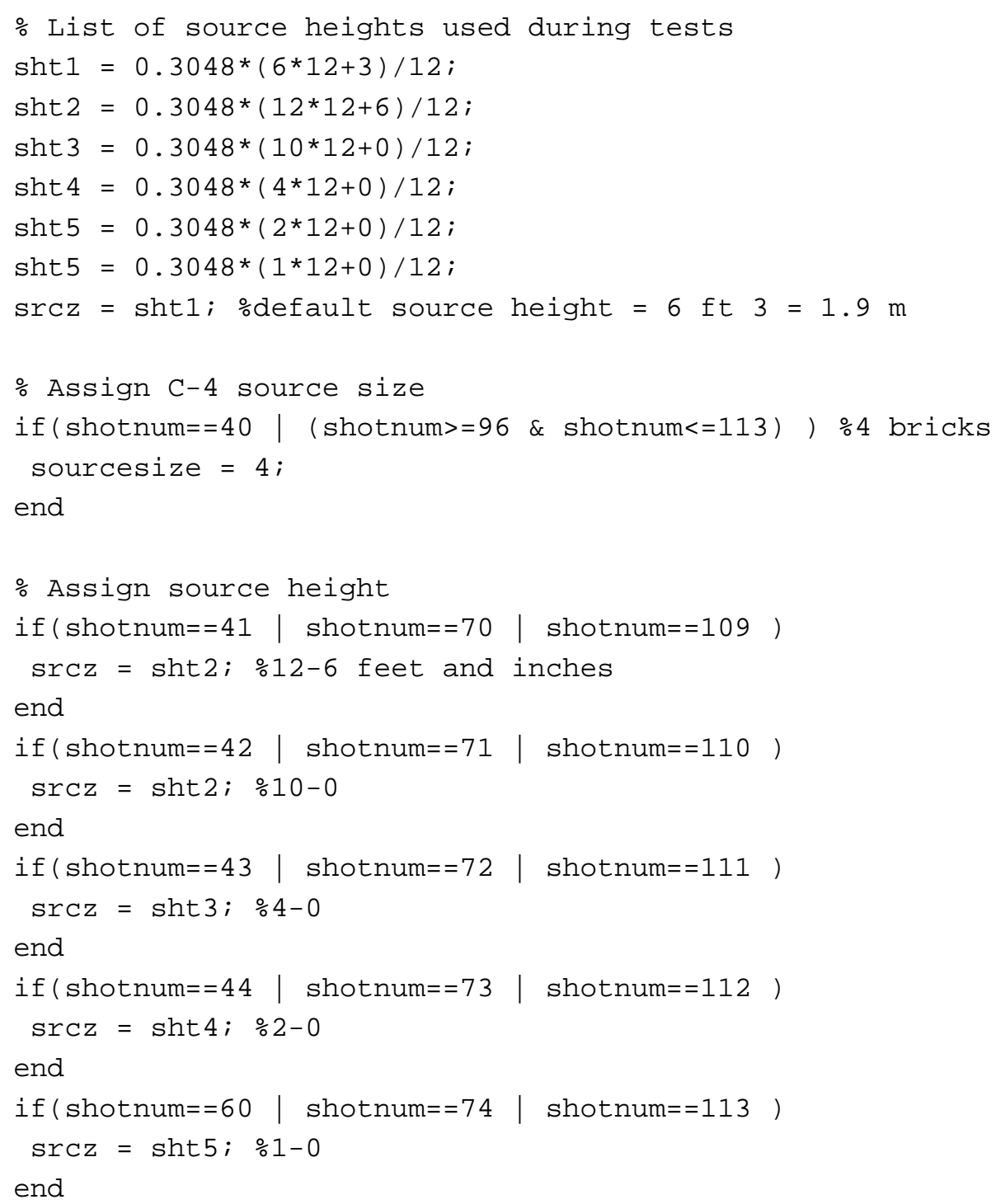




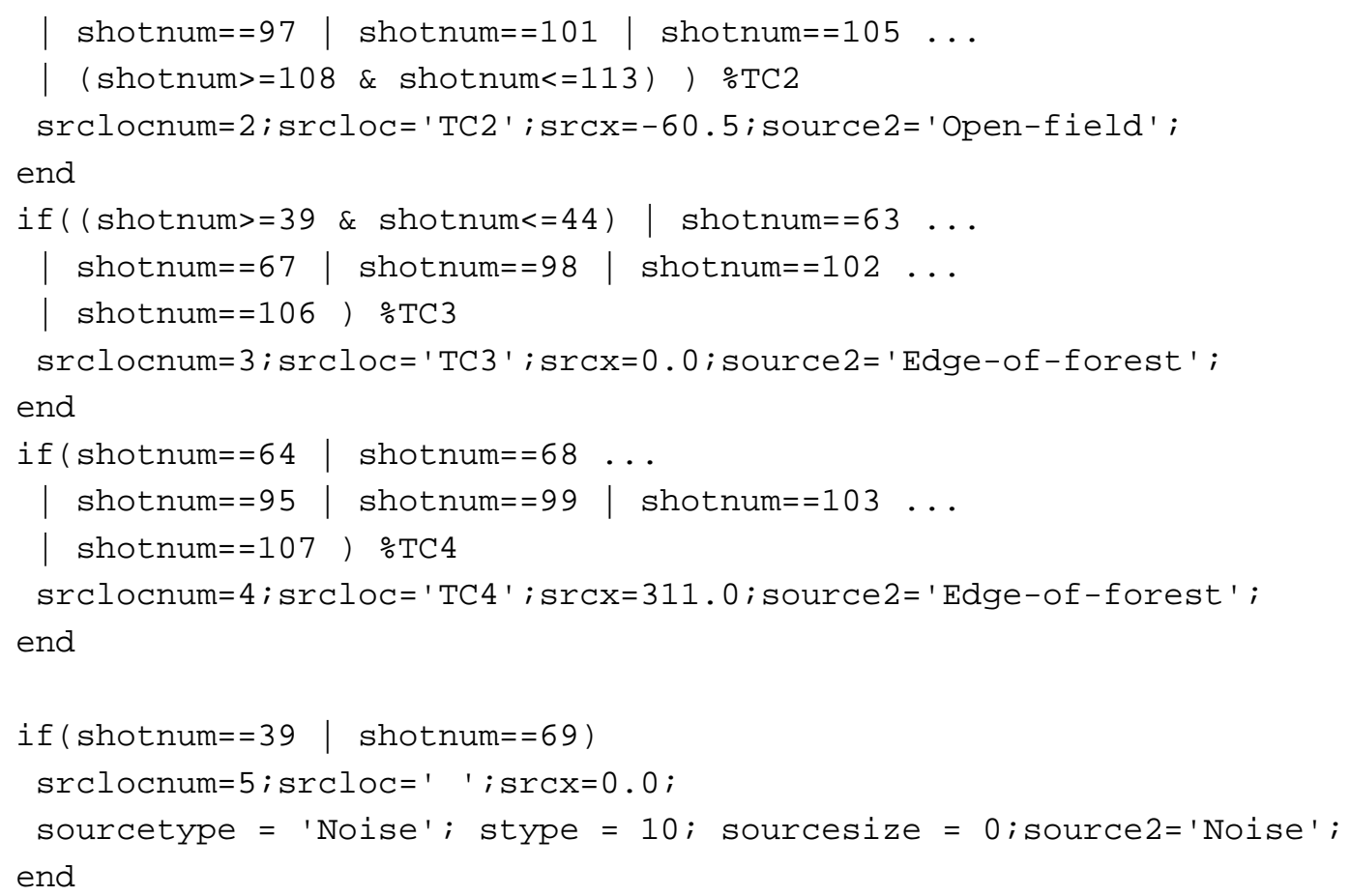

\% Assign source locations for blank pistol shots

if ( shotnum<5) $\% 30 \mathrm{~m}$

srclocnum $=5 ; \operatorname{srcloc}=$ ' $30 \mathrm{~m}$ '; $\operatorname{srcx}=30.0 ; \operatorname{srcy}=2 ; \operatorname{srcz}=1$;

sourcetype $=$ 'Pistol'; stype $=9 ;$, sourcesize $=0 ;$ source $2=$ 'In-forest '; end

if (shotnum $>=5 \&$ shotnum $<10$ ) $\% 90 m$

srclocnum $=7 ;$ srcloc $=$ ' $90 \mathrm{~m}$ '; $\operatorname{srcx}=90.0 ; \operatorname{srcy}=2 ; \operatorname{srcz}=1$;

sourcetype $=$ 'Pistol'; stype $=9 ;$, sourcesize $=0 ;$ source $2=$ 'In-forest' $;$ end

if ( shotnum $>=10$ \& shotnum $<15$ ) $\% 60 \mathrm{~m}$

srclocnum $=6 ;$ srcloc $=$ ' $60 \mathrm{~m}$ '; $\operatorname{srcx}=60.0 ;$ srcy $=2 ; \operatorname{srcz}=1$;

sourcetype $=$ 'Pistol'; stype $=9$; sourcesize $=0 ;$ source $2=$ 'In-forest' ; end

if ( shotnum $>=15 \&$ shotnum $<20$ ) $\% 30 m$

srclocnum $=5 ;$ srcloc $=$ ' $30 \mathrm{~m}$ '; $\operatorname{srcx}=30.0 ; \operatorname{srcy}=2 ; \operatorname{srcz}=1$;

sourcetype $=$ 'Pistol'; stype $=9$; sourcesize $=0$; source $2=$ 'In-forest' ;

end

\% Calculate source-to-sensor distances for this shot srcx $2=\operatorname{srcx}{ }^{*} \operatorname{srcx} ; \operatorname{srcy} 2=$ srcy $^{*}$ srcy; $\operatorname{srcz} 2=\operatorname{srcz}{ }^{*} \operatorname{srcz} ;$ 


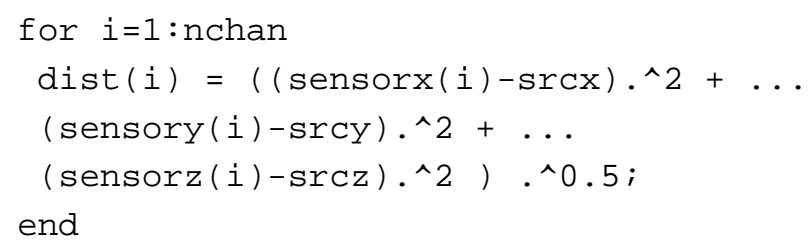


if $($ shotnum $==72)$; cerlrec $=145$ '; end if ( shotnum $==73)$; cerlrec $=' 46^{\prime}$; end if ( shotnum $==74) ;$ cerlrec $=' 47$ '; end if $($ shotnum $==93)$; cerlrec $=' 16^{\prime}$; end if $($ shotnum $==94) ;$ cerlrec $=117$ '; end if ( shotnum==95); cerlrec $=' 19$ '; end $\%$ missed CERL shot 18

if $($ shotnum $==96)$; cerlrec $=' 20$ '; end if $($ shotnum $==97) ;$ cerlrec $=' 21^{\prime}$; end if ( shotnum $==98)$; cerlrec $=' 22$ '; end if $($ shotnum $==99)$; cerlrec $=' 23$ '; end if ( shotnum $==100)$; cerlrec $=$ ' 24 '; end if $($ shotnum $==101)$; cerlrec $=' 25$ '; end if ( shotnum $==102)$; cerlrec $=' 26$ ' ; end if ( shotnum $==103$ ); cerlrec $=' 27$ '; end if ( shotnum $==104) ; \operatorname{cerlrec}=' 28$ '; end if ( shotnum $==105)$; cerlrec $=' 29^{\prime}$; end if ( shotnum $==106)$; cerlrec $=$ ' 30'; end if ( shotnum $==107)$; cerlrec $=' 31$ '; end if ( shotnum $==108)$; cerlrec $=' 32$ '; end if (shotnum==109); cerlrec $=$ ' $33^{\prime}$; end if ( shotnum $==110) ; \operatorname{cerlrec}=' 34$ '; end if ( shotnum==111); cerlrec $=$ ' 35 '; end if ( shotnum $==112)$; cerlrec $=' 36^{\prime}$; end if $($ shotnum $==113) ; \operatorname{cerlrec}=' 37$ '; end $\%$ CRREL Blank Pistol Shots

if $($ shotnum $==1)$; cerlrec $=$ ' 101 '; end if $($ shotnum $==2)$; cerlrec $=' 102$ '; end if $($ shotnum $==3)$; cerlrec $=1103$ '; end if $($ shotnum $==4)$; cerlrec $=' 104$ '; end if $($ shotnum $==5)$; cerlrec $=' 105$ '; end if $($ shotnum $==6)$; cerlrec $=1106$ '; end if $($ shotnum $==7)$; cerlrec $=' 107$ '; end if $($ shotnum $==8)$; cerlrec $=' 108$ '; end if $($ shotnum $==9)$; cerlrec $=' 109$ '; end if $($ shotnum $=10)$; cerlrec $=1110$ '; end if $($ shotnum $==11)$; cerlrec $=' 111$ '; end if $($ shotnum==12); cerlrec $=$ '112'; end if $($ shotnum $==13)$; cerlrec $=' 113$ '; end if $($ shotnum==14); cerlrec $=' 114$ '; end if $($ shotnum $==15) ;$ cerlrec $=' 115$ '; end 


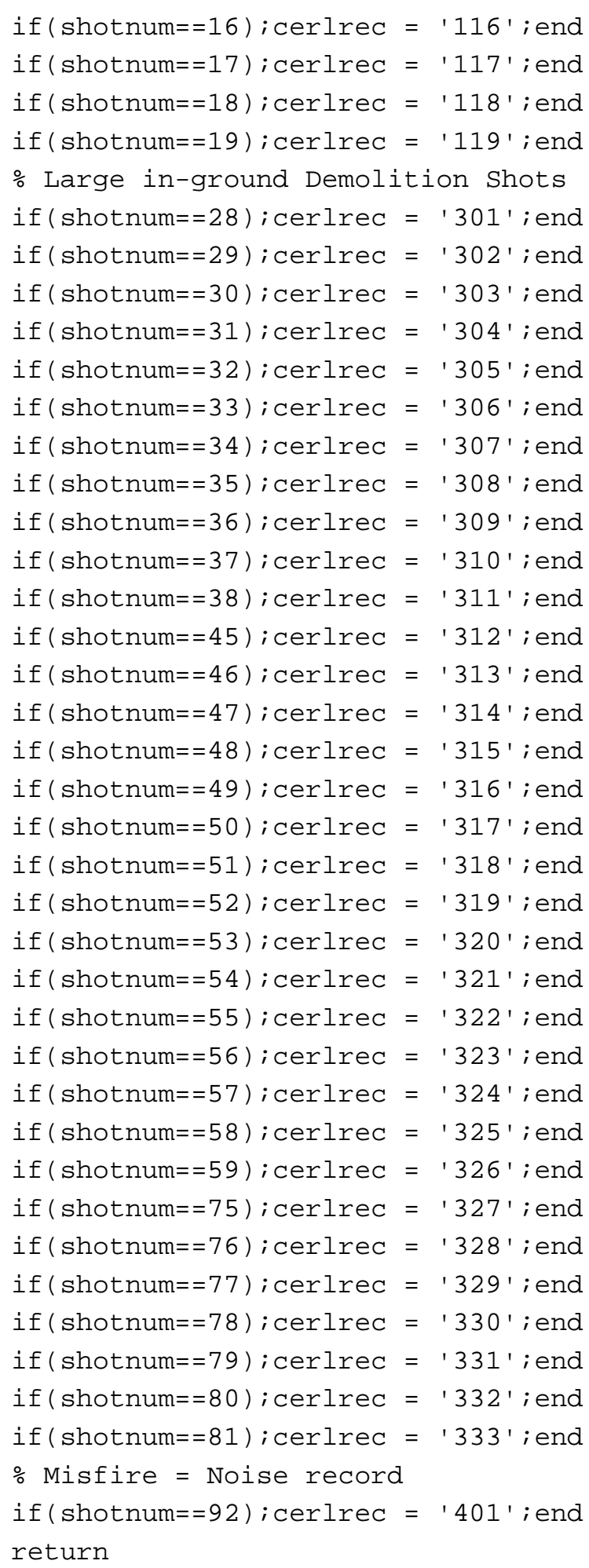




\section{tx02plotnz.m-Three-panel plot of binary seismograph data}

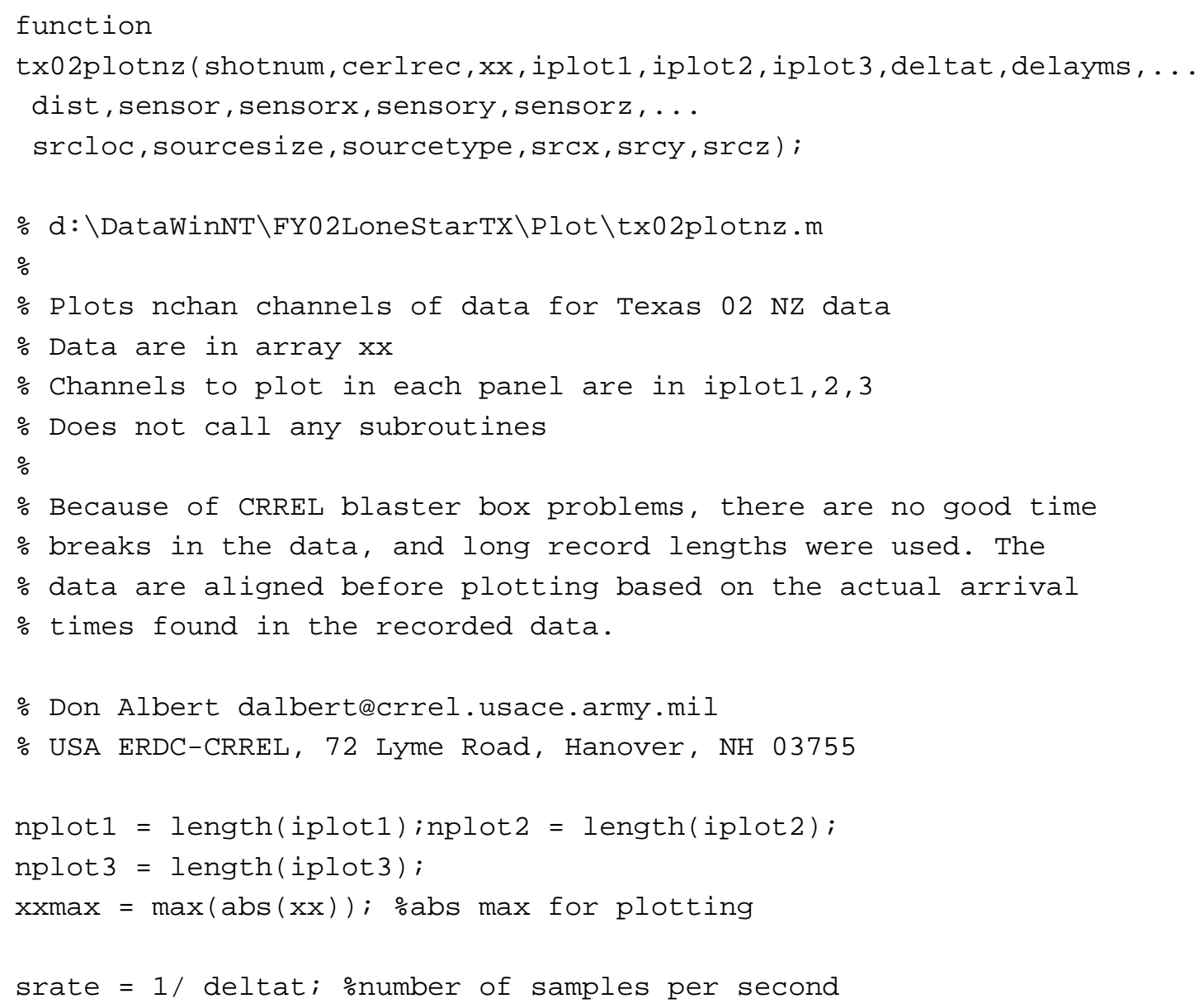




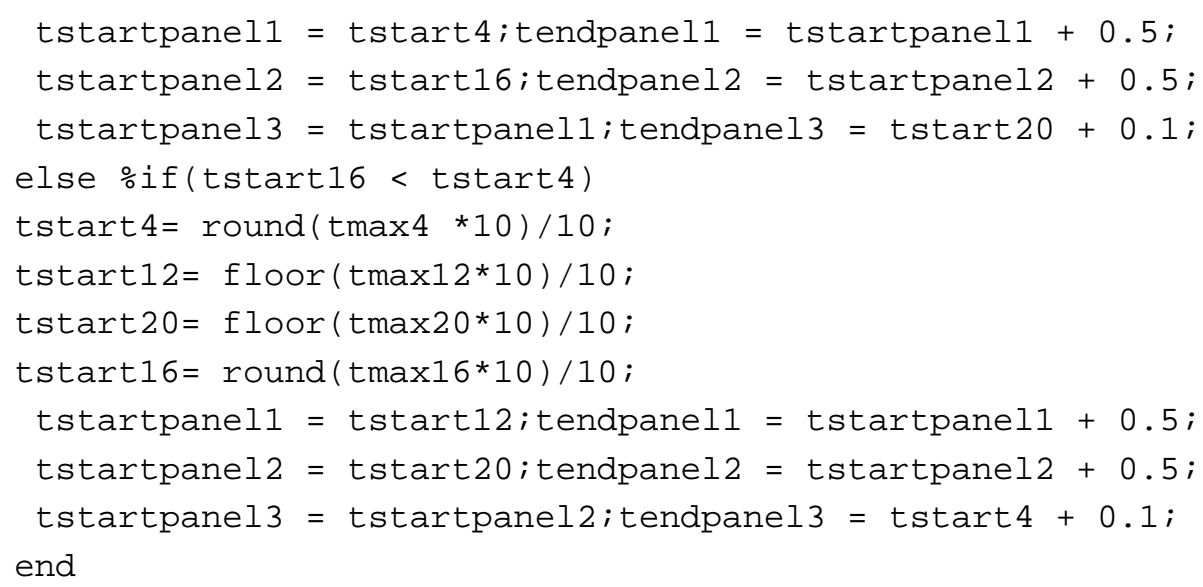


\% Set axis params here; depends on nplot - t2

yshift $=(1:$ nplot $) * 2-2$;

ax $=$ [tstartpanel1-0.05 tendpanel1 $-122^{*}$ nplot $]$;

$\operatorname{axis}(\mathrm{ax})$;

ax1 = gca; $\operatorname{set}(a \times 1$, 'YTick', [] $) ; \operatorname{set}(a \times 1$, 'Box', 'on' $)$;

xlabel('Time, sec')

$\%$ plot data and label

hold on;

for $i=1: n p l o t$

plot $\left(t,(x x(1: \operatorname{length}(t), \operatorname{iplot}(i)) / x x \max (i p l o t(i)))+y \operatorname{shift}(i), k^{\prime}\right)$;

leftlabel $=\operatorname{sprintf}\left({ }^{\prime} \% g^{\prime}, \operatorname{round}(\operatorname{dist}(\operatorname{iplot}(i)))\right)$;

$\%$ Text labels for plot

str1 = sensor $($ iplot $(i),:)$;

str2 $=\operatorname{sprintf}\left(' \% 7.2 e^{\prime},(\operatorname{xxmax}(i p l o t(i)))\right)$;

if $(x x m a x(i p l o t(i)))<0.0001$

str $2=\operatorname{sprintf}\left({ }^{\prime} \% 4.1 \mathrm{fE}-6\right.$ ', $\left.\left(1 \mathrm{E} 6{ }^{*} \times x \max (\operatorname{iplot}(i))\right)\right)$;

elseif $(\operatorname{xxmax}(i p l o t(i)))<0.001$

str2=sprintf ('\%4.2fE-3', (1E3*xxmax (iplot(i))));

elseif $(x \operatorname{xmax}($ iplot $(i)))<0.1$

str2=sprintf $($ '\%4.1fE-3', (1E3*xxmax (iplot (i))) );

elseif $(x \operatorname{xmax}(\operatorname{iplot}(i)))<10.0$

str2=sprintf $\left(' \% 4.2 f^{\prime},(x \operatorname{xmax}(\operatorname{iplot}(i)))\right)$;

elseif $(x \operatorname{xmax}($ iplot $(i)))>1000.0$

str2=sprintf('\%4.1fk',( $\operatorname{xxmax}(\operatorname{iplot}(i)) / 1000))$;

else

str2=sprintf $\left(' \% 4.1 f^{\prime},(\operatorname{xxmax}(\operatorname{iplot}(i)))\right)$;

end \%text loop

$\operatorname{str} 3=\operatorname{char}(\operatorname{str} 1)$;

str4 = $\operatorname{char}(\operatorname{str} 3, \operatorname{str} 2)$;

str5 = cellstr (str 4$)$;

text (tendpanel1+0.02, [yshift(i)], str5, 'FontSize ' , 8, ...

'HorizontalAlignment ', ' left ');

end \%plot loop $i=1$ :nplot

subplot $(1,3,2)$; \%Center (2nd) panel of plot

iplot=iplot2; nplot=nplot2;

yshift $=(1:$ nplot $) * 2-2$;

ax $=$ [tstartpanel2-0.05 tendpanel2 $-12^{*}$ nplot];

$\operatorname{axis}(\mathrm{ax})$;

ax1 = gca; $\operatorname{set}(a \times 1$, 'YTick', [] $) ; \operatorname{set}(a \times 1$, 'Box', 'on' $)$; 


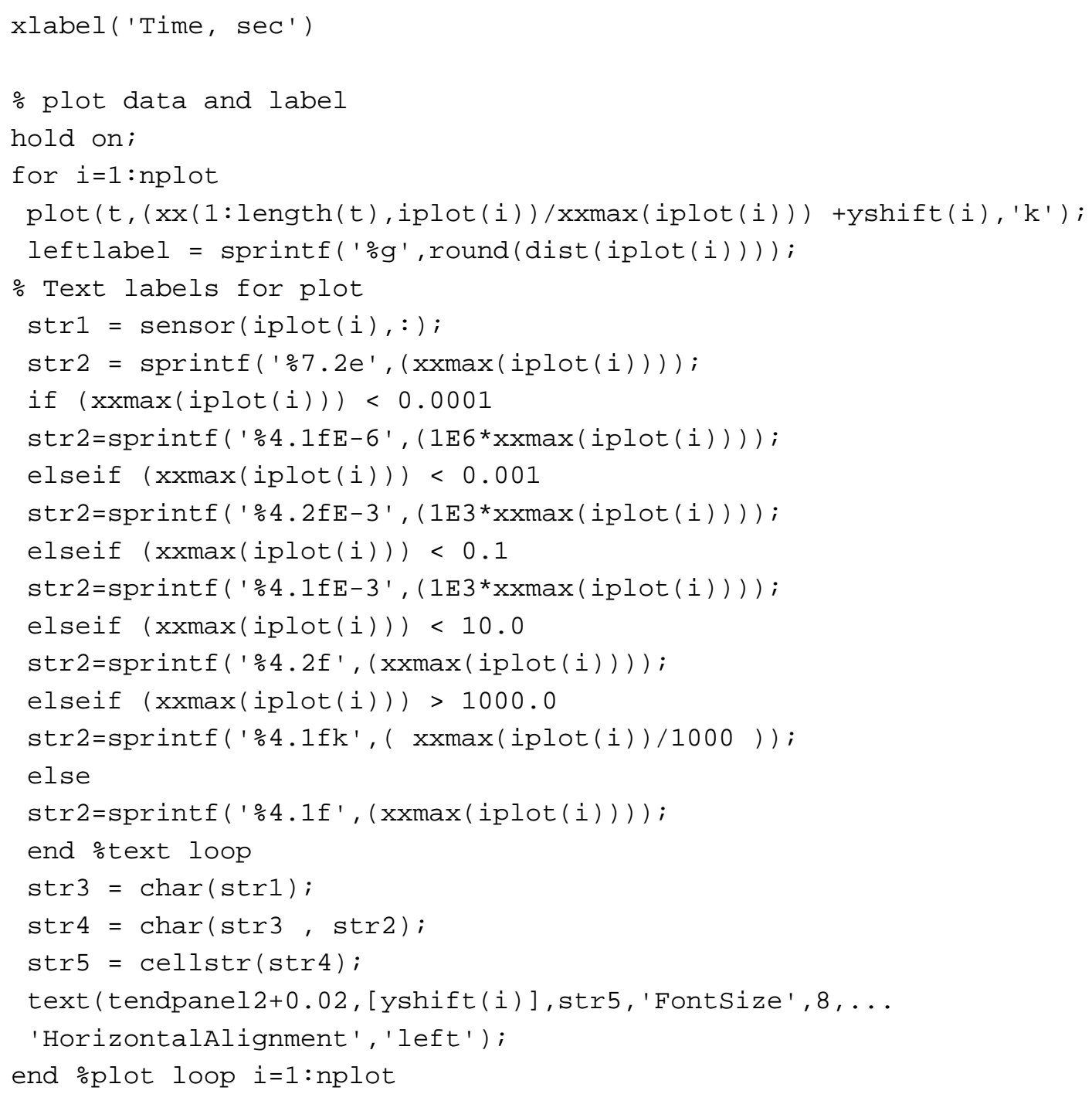


hold on;

for $i=1: n p l o t$

if $(x \operatorname{xmax}(\operatorname{iplot}(i)==0)) ; x x \operatorname{xax}(\operatorname{iplot}(i))=1$; end

$\operatorname{plot}\left(t,(x x(1: \operatorname{length}(t), \operatorname{iplot}(i)) / x x m a x(i p l o t(i)))+y \operatorname{shift}(i), k^{\prime}\right)$;

leftlabel $=\operatorname{sprintf}\left({ }^{\prime} \% g^{\prime}, \operatorname{round}(\operatorname{dist}(\operatorname{iplot}(i)))\right)$;

$\%$ Text labels for plot

str1 = sensor $(i p l o t(i),:)$;

str2 $=\operatorname{sprintf}\left({ }^{2} 7.2 \mathrm{e}^{\prime},(\operatorname{xxmax}(\operatorname{iplot}(i)))\right)$;

if $(x x \operatorname{xax}(\operatorname{iplot}(i)))<0.0001$

str2=sprintf( '\%4.1fE-6', (1E6*xxmax (iplot(i))));

elseif $(x \operatorname{xmax}(i p l o t(i)))<0.001$

str2=sprintf $($ '\%4.2fE-3', (1E3*xxmax (iplot(i))));

elseif $(\operatorname{xxmax}($ iplot $(i)))<0.1$

str2=sprintf ('\%4.1fE-3',(1E3*xxmax (iplot(i))));

elseif $(x \operatorname{xmax}(\operatorname{iplot}(i)))<10.0$

str $2=\operatorname{sprintf}\left({ }^{\prime} \% 4.2 f^{\prime},(x \operatorname{xmax}(\operatorname{iplot}(i)))\right)$;

elseif $(x \operatorname{xmax}($ iplot $(i)))>1000.0$

str2=sprintf ('\%4.1fk',( $x x \max (i p l o t(i)) / 1000))$;

else

str2=sprintf ('\%4.1f', (xxmax (iplot (i))) );

end \%text loop

$\operatorname{str} 3=\operatorname{char}(\operatorname{str} 1)$;

str $4=\operatorname{char}(\operatorname{str} 3, \operatorname{str} 2) ;$

str5 = cellstr $($ str 4$)$;

text (tendpane13+0.02, [yshift(i)], str5, 'FontSize' , 8, ...

'HorizontalAlignment', 'left');

$\%$ Write title for right panel

if $(i==n p l o t)$

text(tstartpane13+0.05,yshift(i)+1, 'Pressure Sensors') ;

end

end \%plot loop $i=1$ :nplot

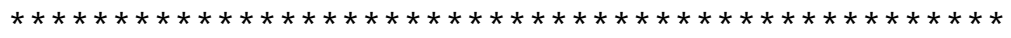

\% Title for entire plot

- - - - . - .

if (sourcesize $=0$ ) \% C-4 shot

plottitle $=\ldots$

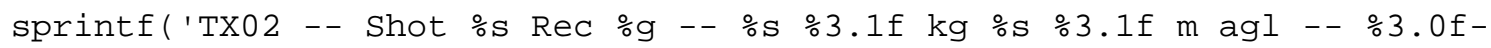
$\% 3.0 f \mathrm{~m}^{\prime}, \ldots$ 
cerlrec, shotnum, srcloc, sourcesize*1.25/2.2, sourcetype, srcz, min(dist), max (dist));

else \%not C-4 shot

plottitle $=\ldots$

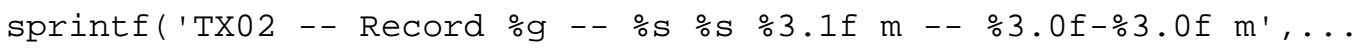
shotnum, srcloc, sourcetype, srcz, $\min ($ dist), $\max ($ dist )) ;

end

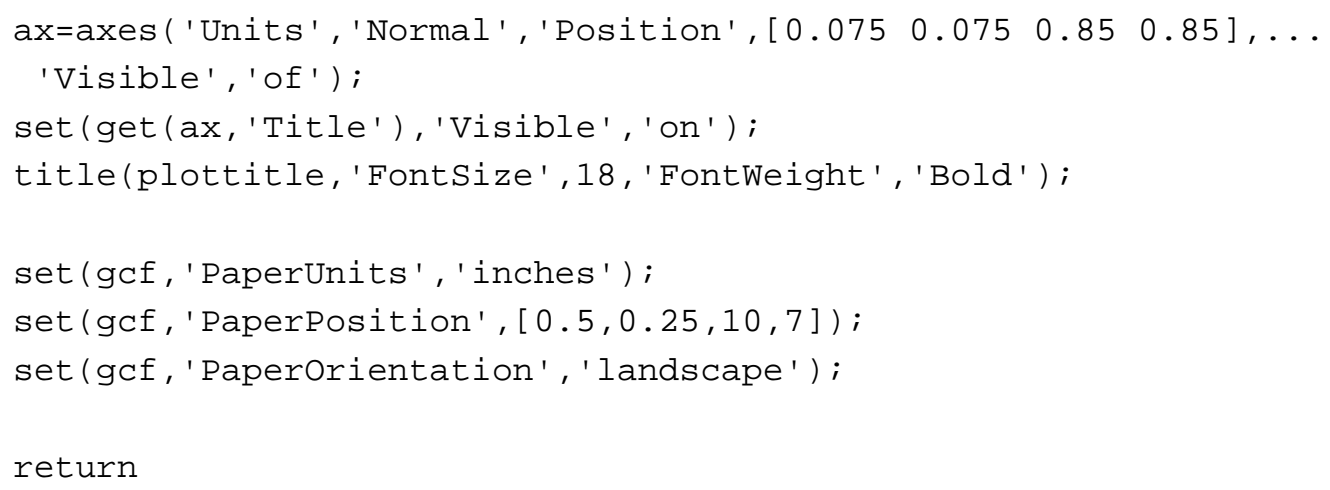

\section{tx02tbcorr-Finds true shot instant}

function [itimeTB, itime $\odot$, itime1, time $\odot, x d i s t, x t i m e]=$ txe2tbcorr (shotnum, ... 
$\%$ time 0 is the start time for saving the time series

\% Don Albert dalbert@crrel.usace.army.mil

\% USA ERDC-CRREL, 72 Lyme Road, Hanover, NH 03755

fid13=fopen ( 'Tx02TimeBreakLog.m', ' a' ) ;

srate $=1 /$ deltat $; \%$ number of samples per second

\% Pressure channels to process

$\left[\begin{array}{ll}a & b\end{array}\right]=\max \left(x x\left(:,\left[\begin{array}{lllll}4 & 8 & 12 & 16 & 20\end{array}\right]\right)\right)$;

$\mathrm{b} 2=\mathrm{b}+$ delayms*srate $/ 1000 ; \mathrm{b} 2=\mathrm{b} 2 /$ srate $;$

$x p=\left[\begin{array}{lllll}30 & 60 & 90 & 120 & 150\end{array}\right] ;$

if $(\operatorname{srcloc}(3)==' 11)$;

$x p=x p+445-30 ; \% \mathrm{TC} 1$

elseif $\left(\operatorname{srcloc}(3)==2^{\prime}\right.$ ' $)$

$x p=x p+91-30 ; \% \mathrm{TC2}$

elseif $\left(\operatorname{srcloc}(3)==3^{\prime}\right.$ ')

$x p=x p+0 ; \% \mathrm{TC} 3$

elseif $\left(\operatorname{srcloc}(3)==\right.$ ' $^{\prime}$ ')

$x p=\left[\begin{array}{lllll}281 & 251 & 221 & 191 & 161\end{array}\right] ; \% T C 4$

else

$x p=\left[\begin{array}{lllll}30 & 6 \odot & 90 & 120 & 150\end{array}\right] ;$

end

vel $=(x p(5)-x p(1)) /(b 2(5)-b 2(1)) ;$

t1actual $=x p(1) / v e l$;

tocorr = t1actual - b2(1);

b3 = b2 + tocorr; \%Corrected travel times

\% Code for pistol shots only - uses different sensors

if( (shotnum <= 19); \%Blank pistol shots

if(shotnum <=4) \%pistol at $30 \mathrm{~m}$

iii1 $=1$; iii2 = 3; \%start chan 4 , end chan 12

elseif(shotnum $>4$ \& shotnum $<9$ ) \%pistol at $90 \mathrm{~m}$

iii1 $=3$; iii2 $=5$; \%start chan 12, end chan 20

elseif(shotnum $>9$ \& shotnum<14) \%pistol at $60 \mathrm{~m}$

iii1 $=2 ;$ iii2 $=4 ;$ sstart chan 8 , end chan 16 


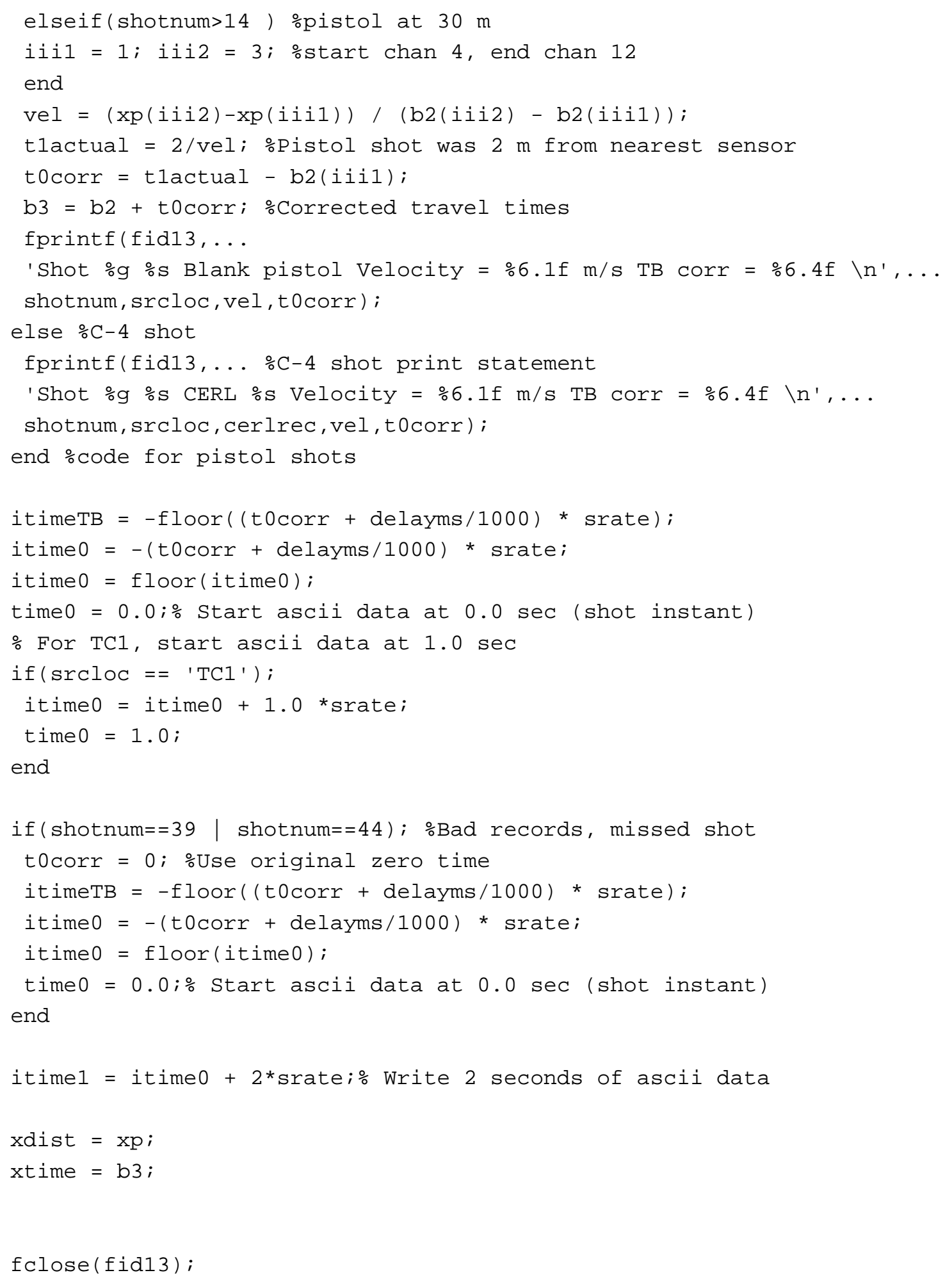


return

\section{tx02writenchan.m - Writes multichannel ASCII file tx02nnn.asc}

function tx@2writenchan(shotnum, cerlrec, $x x$, npts, deltat, nchan, delayms, ... descalingfact, ... stackcount, acqdate, acqtime, engfact, xgain, itime $\odot$, itime1, time $\odot, \ldots$ dist, sensor, rtype, sensorx, sensory, sensorz, sensor $2, \ldots$

srcloc, stype, sourcetype, sourcesize, srcx, srcy, srcz, source2);

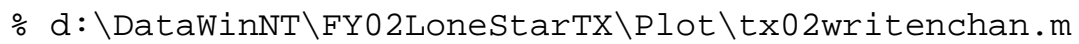

$\%$ Writes nchan (24) channel ascii file for Texas 2002 data

$\%$ File name is t $x 02 x x x$.asc, where $x x x$ is a new file number

$\% x x x=C E R L$ Shot number if it was a $C-4$ shot

$\%$ Calls no subroutines

\% Don Albert dalbert@crrel.usace.army.mil

\% USA ERDC-CRREL, 72 Lyme Road, Hanover, NH 03755

srate $=1 /$ deltat; $\%$ number of samples per second

recnum $=$ shotnum; npts $=$ length $(x x)$;

stype $=1 ;$ signal=1; srccomment=source $2 ;$

if $($ shotnum $<20) ;$ stype $=5 ;$ signal $=0 ;$ end

comment $=$ 'Lone Star AAP, Texarkana, TX Blast Noise Through Forest

W/CERL' ;

\% Construct ascii file name and open

cerlrec2 = str2num $($ cerlrec $)+2000$;

if (cerlrec $=0)$;

fname2 = sprintf('txœ\%g.asc', cerlrec2); \%tx०2001 - tx®2037

else

if $($ recnum<10)

fname2 = sprintf('tx@2spistol@\%s.asc', num2str(recnum)); \%tx@2pistol01 09

else

fname2 $=\operatorname{sprintf}($ 'tx02pistol\%s.asc', num2str $($ recnum $)) ; \%$ tx@2pistol10 19

end

end

fid5=fopen (fname2, ' $w$ ') ; 


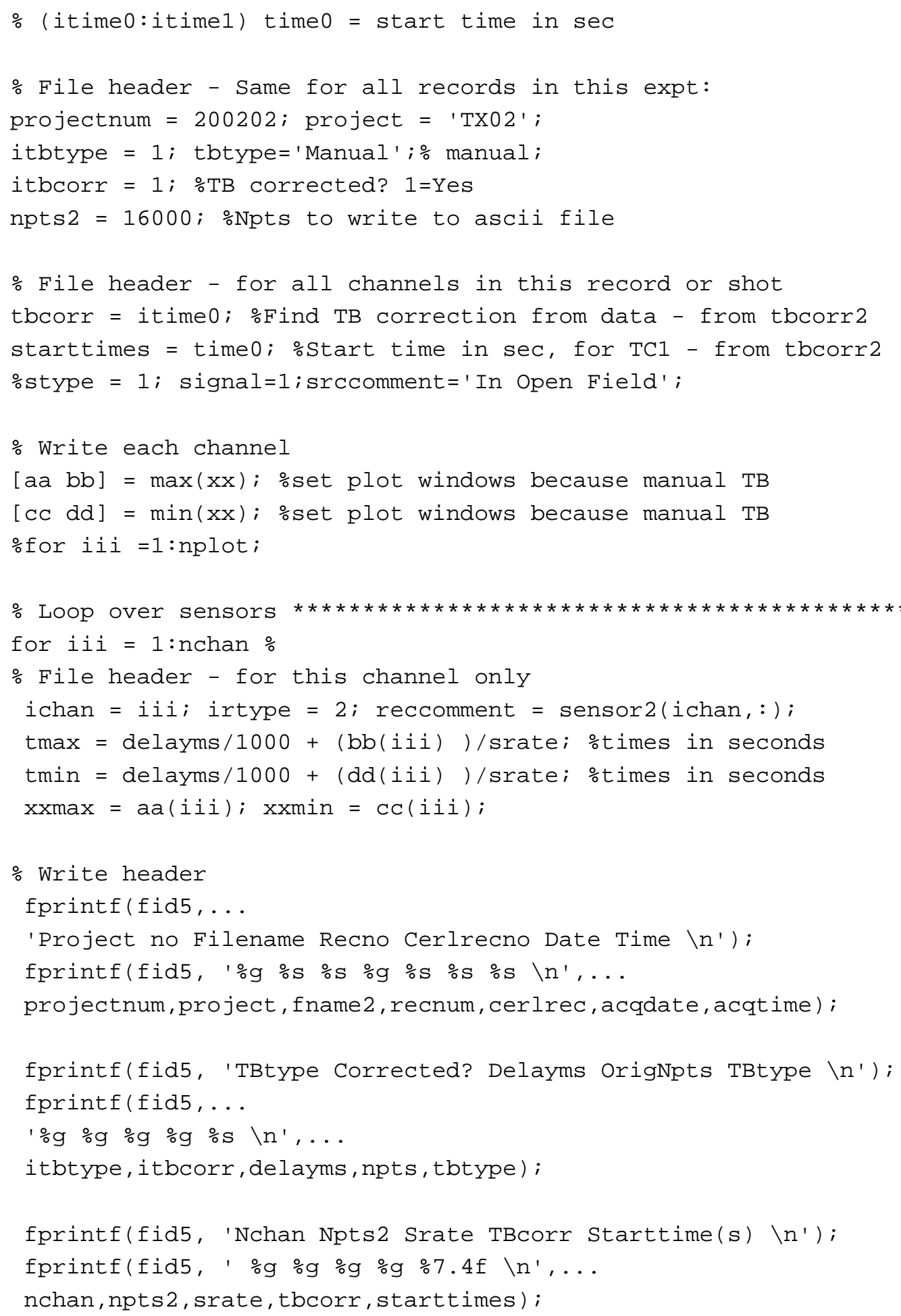




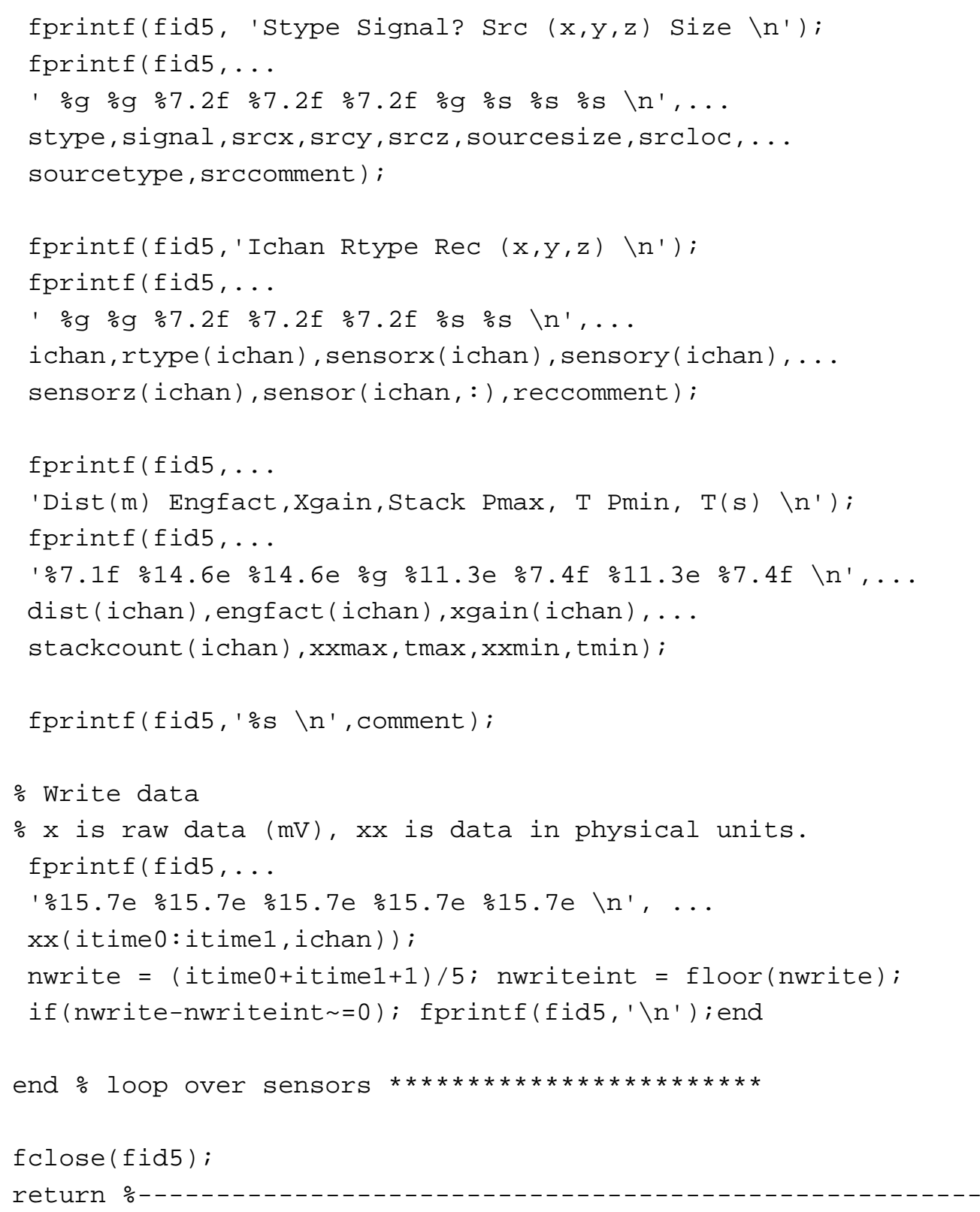

readnz.m-Reads NZ seismograph binary data file

function [scan, samplesPerScan, samplingInterval, nbofTraces, ... delaytime, descalingfact, stackcount, acqdate, acqtime] = readnz (filename)

\% Reads a binary seismograph file in SEG-2 format, and returns the 
$\%$ data in "scan" variable above.

\% This code written by Don Albert, CRREL, and based on code "SEG2LOAD" written

$\%$ by Pièce PY to read a radar file in modified SEG-2 format. Some of Piece's

$\%$ original code is still here in this file.

$\%$ Calls subroutine words.m

$\%$ Writes info about the file to file readnzlog.m

\% To read one NZ file directly:

$\%$ fname $=$ '10077.dat';

$\%[x$, npts, deltat, nchan, npts2, delayms, descalingfact, acqdate, ...

$\%$ acqtime $]=\operatorname{readnz}($ fname $)$;

\% READNZ - For Geometrics NZ seismograph

\%This version modified from MOUT data for MNO2 data

\% READNZ Modified by D Albert to read standard SEG-2 format

\% SEG2LOAD Read a SEG-2 (standard SEG-2 format of the Society of

\% Exploration Geophysicist) file from disk.

\% [scan, samplesPerScan, samplingInterval, shaft Interval,

$\%$ timerFrequency] =seg2load ('filename') reads the file 'filename'

$\%$ and returns the image scan $[\mathrm{m}, \mathrm{n}]$ containing $\mathrm{n}$ A-Scan of $\mathrm{m}$ samples.

$\%$ If no extension is given for the filename, the extension

$\%$ '.sg2' is assumed.

$\%$ samplesPerScan contains the number of samples per A-scan

$\%$ shaftinterval contains the distance between shaft encoder

$\%$ triggers in meter

\% samplingInterval contains the time between 2 samples in pico-seconds

$\%$ timerFrequency contains the frequency of A-scan sampling in $\mathrm{Hz}$

$\%$

\% Pièce PY 24/07/1996

$\%$ LAMI - DeTeC Demining Technology Center

\% Swiss Federal Institute of Technology (EPFL) -

\% Lausanne, Switzerland

\% Don Albert dalbert@crrel.usace.army.mil

\% USA ERDC-CRREL, 72 Lyme Road, Hanover, NH 03755

$\%$ check argument and filename

if (nargin $==0$ ) 


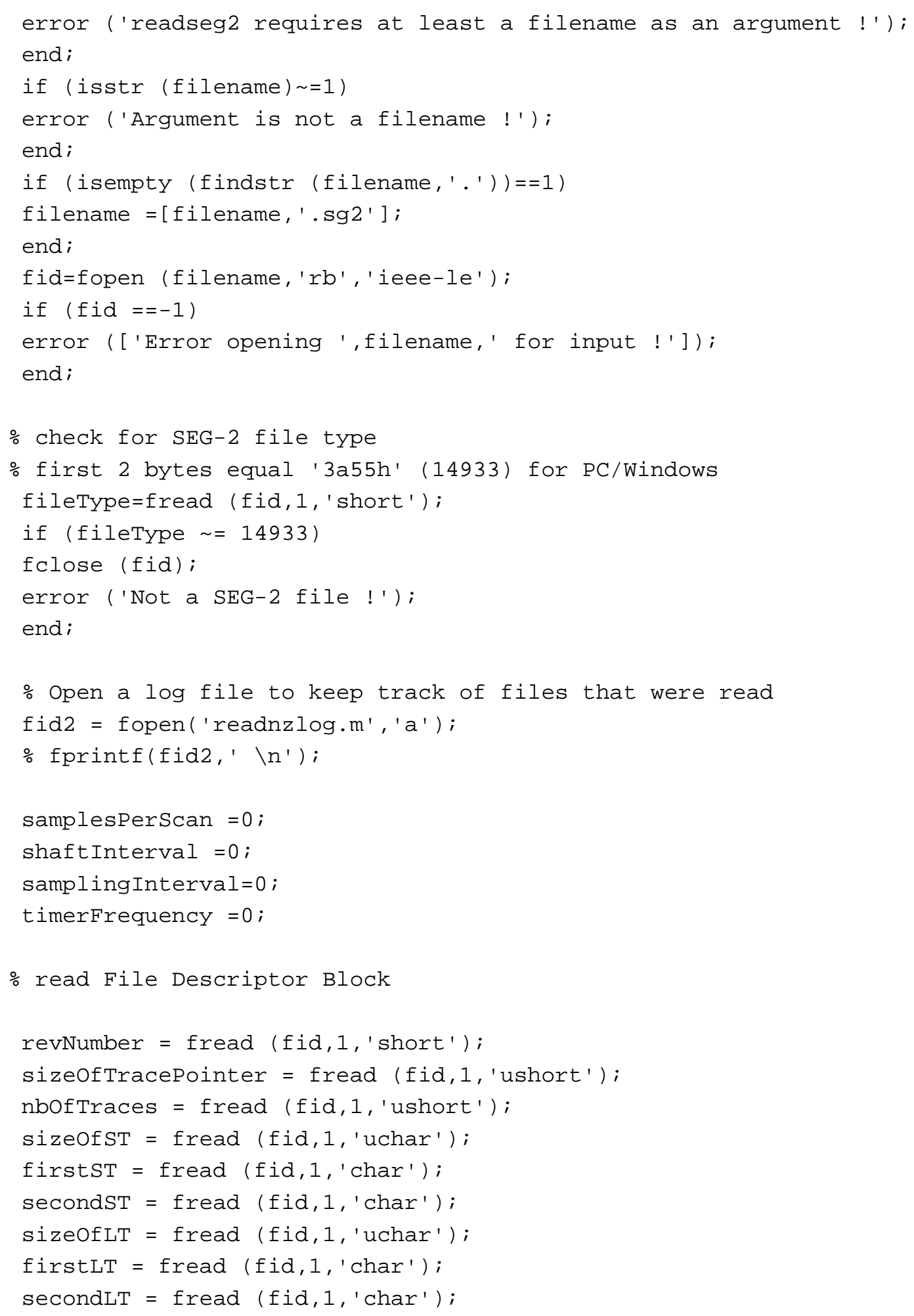




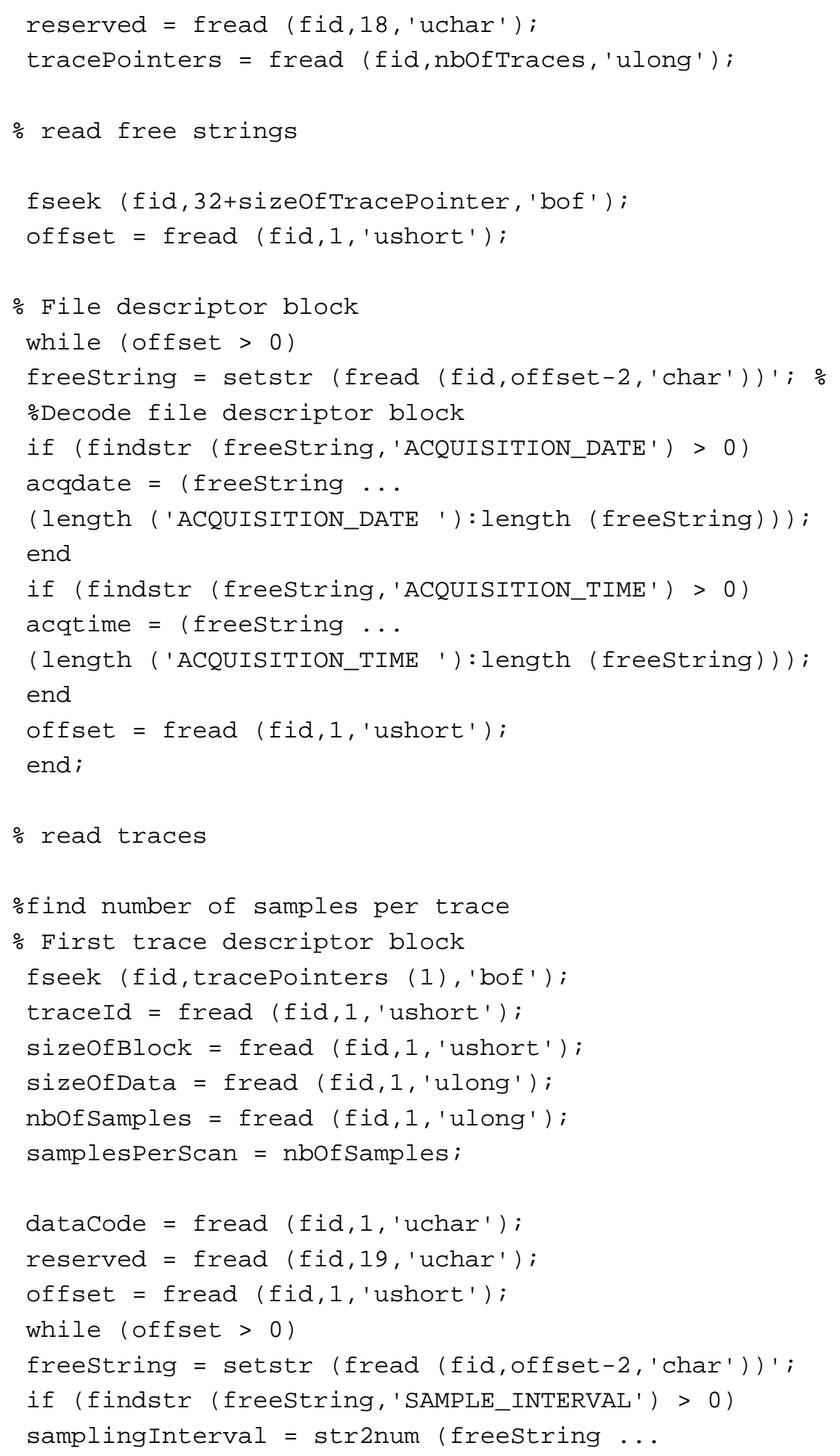




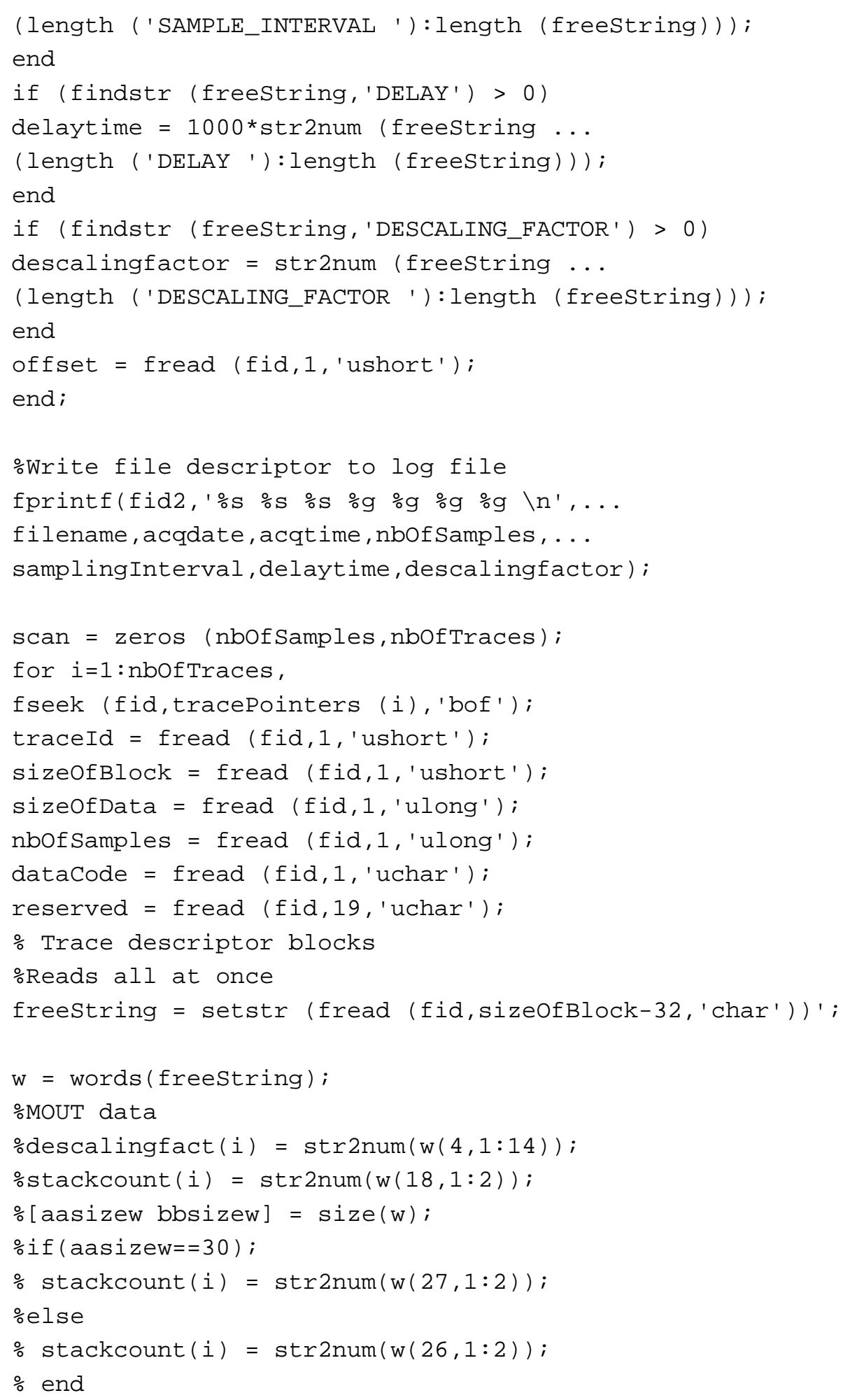




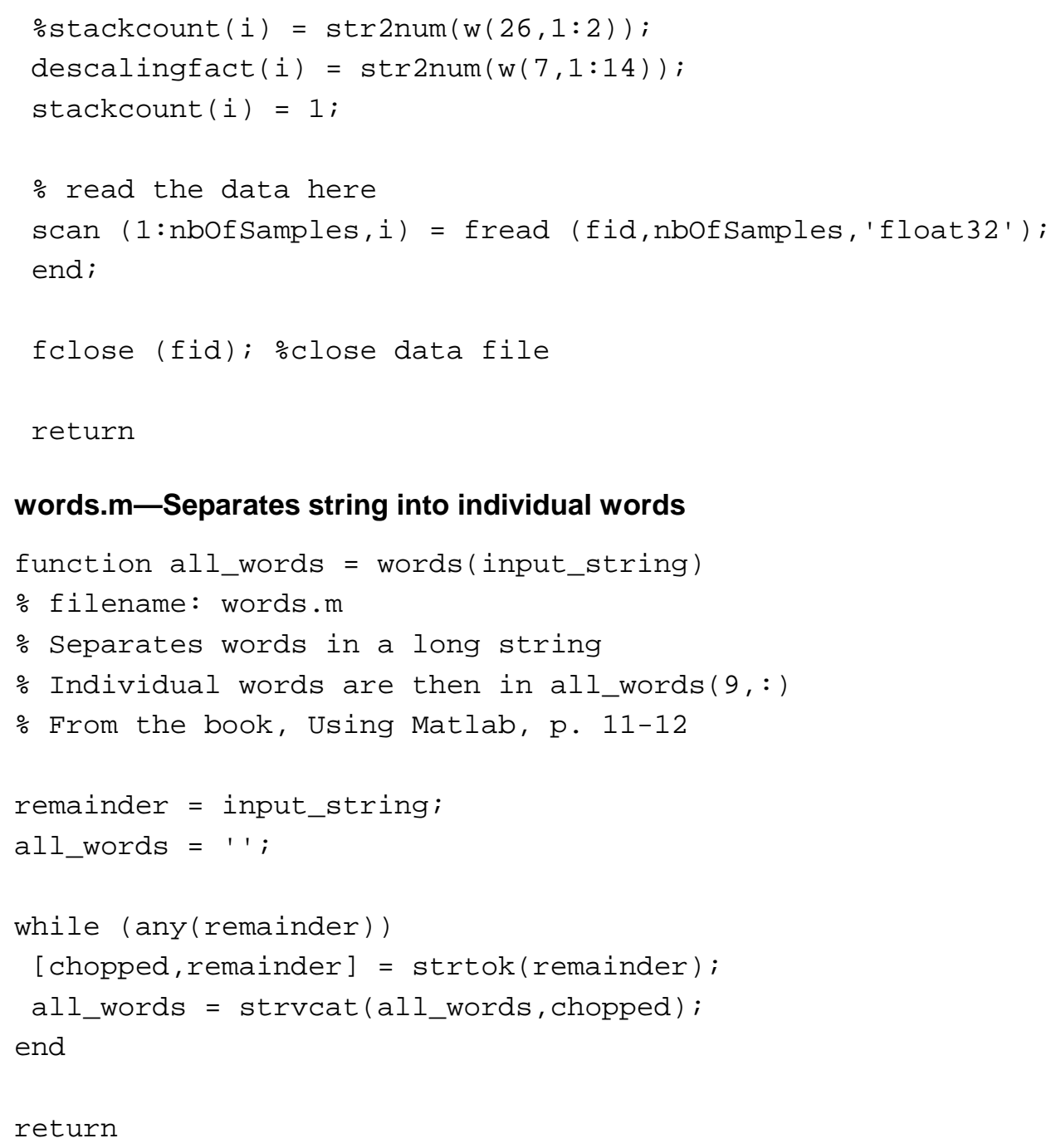




\section{APPENDIX C: MATLAB PROGRAMS TO READ AND PLOT MULTICHANNEL ASCII DATA FILES.}

This section provides a listing of the MATLAB programs used to read and plot the 24 channel ASCII data files that were constructed from the binary files using the programs in the previous section. The files that are listed in this section are:

9. doplotasc.m - Main program to read multichannel ASCII file and make a three-panel plot of the data. To use, set the desired ASCII file numbers in the variable "recs" and run the program. You may have to change the lines that construct the filenames to use the correct directory for your computer, and you may want to change the print options at the very end of this program to match your printer or delete the plot from the screen after plotting. Calls the programs listed below.

10. readascii.m - Function to read in the data from the multichannel ASCII files. Calls function words.m

11. plotasc.m - Function to make a three-panel plot of the data in landscape orientation. The channels plotted in each of the panels are given by the contents of iplot1, iplot2, and iplot3.

12. words.m - Function to parse a string into individual words. This function is listed in the previous section.

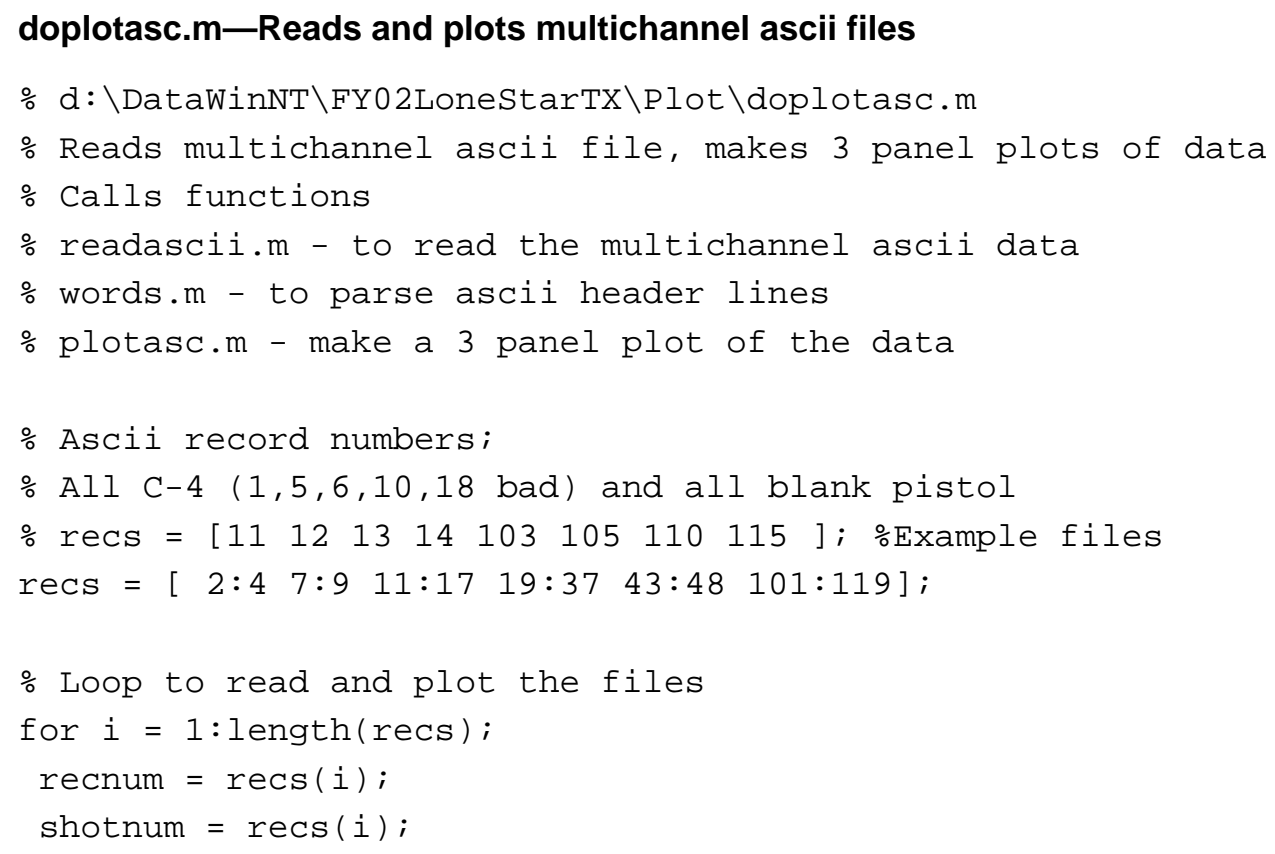




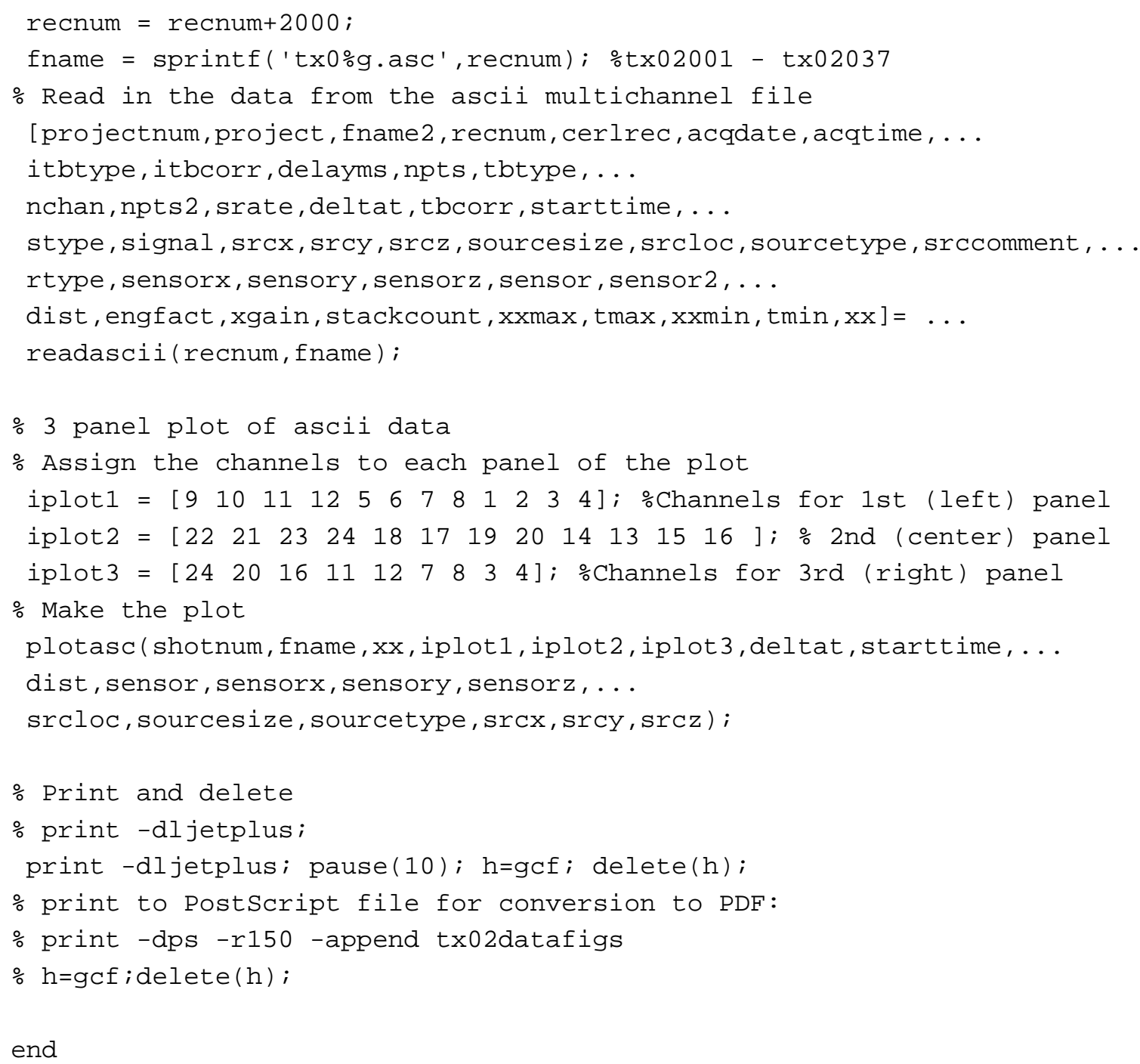




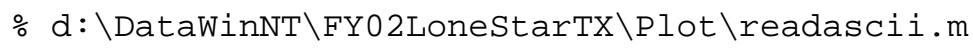

$\%$ Reads in ascii seismograph data from fid6

\% Calls words.m - parse string into individual words

\% Don Albert dalbert@crrel.usace.army.mil

\% USA ERDC-CRREL, 72 Lyme Road, Hanover, NH 03755

$\%$ Read first header to get number of channels

fid6=fopen ( fname, ' $r$ ') ;

line1 = fgets $($ fid6); $\%$ Project no Filename Recno Cerlrecno Date Time

line2 $=$ fgets $($ fid6) ;

line3 $=$ fgets $($ fid6);\%TBtype Corrected? Delayms Orignpts TBtype

line4 = fgets $($ fid6);

line5 $=$ fgets $($ fid6) $; \%$ Nchan Npts2 Srate TBcorr Starttime(s)

line $6=$ fgets $($ fid6) ;

line $7=$ fgets $(f i d 6) ; \%$ Stype Signal? $\operatorname{Src}(x, y, z)$ Size

line8 $=$ fgets $($ fid 6$)$;

line9 $=$ fgets $($ fid6 $) ; \%$ Ichan Rtype $\operatorname{Rec}(x, y, z)$

line10 $=$ fgets $($ fid 6$)$;

line11 = fgets $($ fid6) $;$ \%ist $(\mathrm{m})$ Engfact, Xgain, Stack Pmax, $T$ Pmin, $T(\mathrm{~s})$

line12 = fgets $($ fid6);

line13 = fgets $($ fid6 $) ; \%$ Comment

a6 = words (line6);

nchan $=\operatorname{str} 2 \operatorname{num}(a 6(1,:)) ; \operatorname{npts} 2=\operatorname{str} 2 \operatorname{num}(a 6(2,:))$;

fid6=fopen (fname, ' $r$ '); \%rewind file

for iii $=1:$ nchan $\%$ Read data

$\%$ Read header - Same for all records in this expt:

line1 $=$ fgets $($ fid6 $) ;$ line2 $=$ fgets $($ fid 6$) ;$ line3 $=$ fgets $($ fid6 $) ;$

line4 $=$ fgets $($ fid6 $) ;$ line5 $=$ fgets $($ fid 6$) ;$ line $6=\operatorname{fgets}($ fid 6$)$;

line $7=$ fgets $($ fid6 $) ;$ line8 $=$ fgets $($ fid 6$) ;$ line $=$ fgets $($ fid6 $) ;$

line10 $=$ fgets $($ fid6 $) ; \operatorname{line11}=$ fgets $($ fid6 $) ;$ line12 = fgets $($ fid6 $)$;

line13 = fgets $($ fid6 $) ; \%$ Comment

$\%$ decode header

a2 $=$ words(line $) ;$ a4 $=$ words (line $) ;$ a $6=\operatorname{words}(l$ ine 6$) ;$

a8 $=$ words $($ line 8$) ;$ a10 $=$ words $($ line 10$) ;$ a12 = words $(l i n e 12)$; 


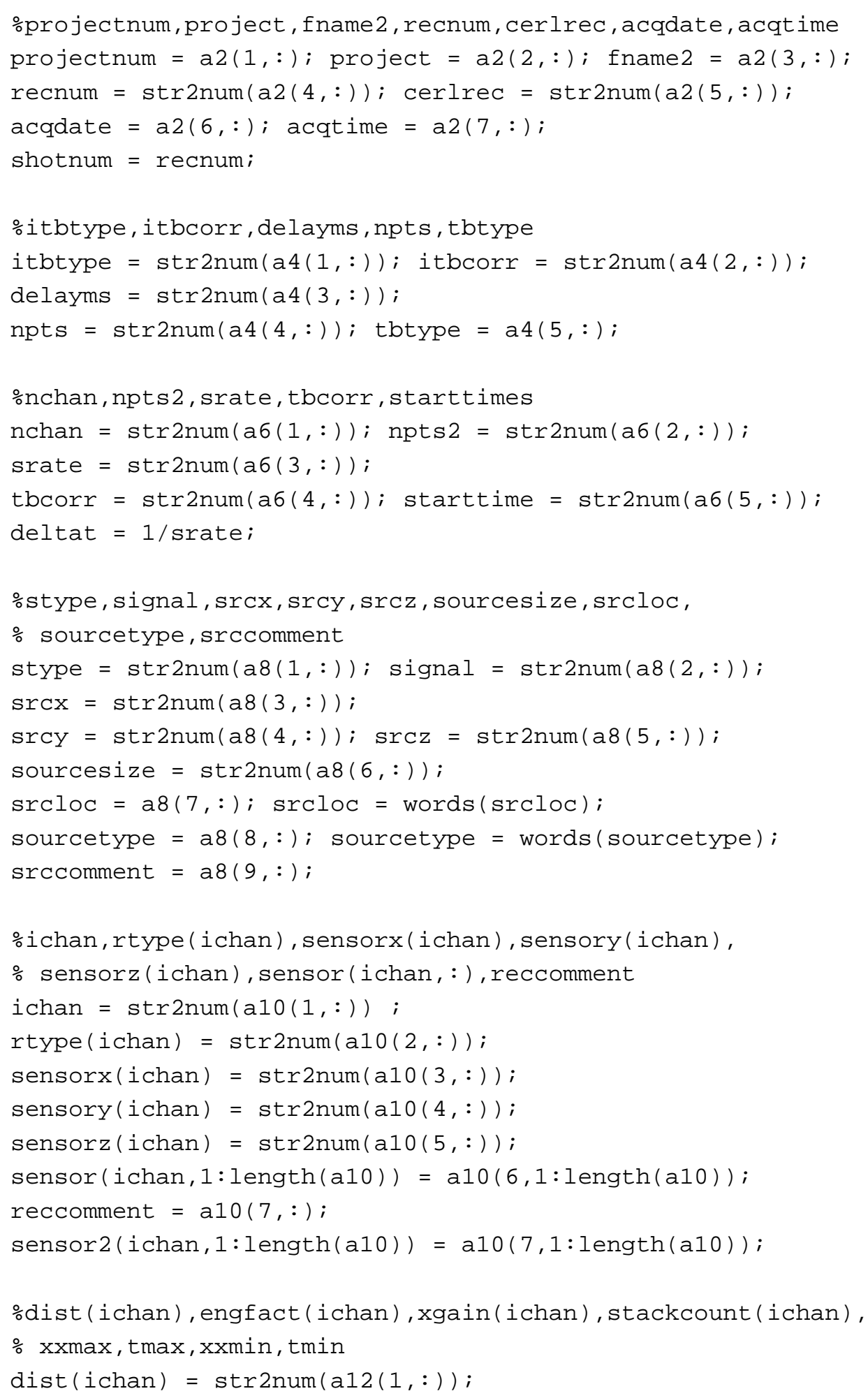




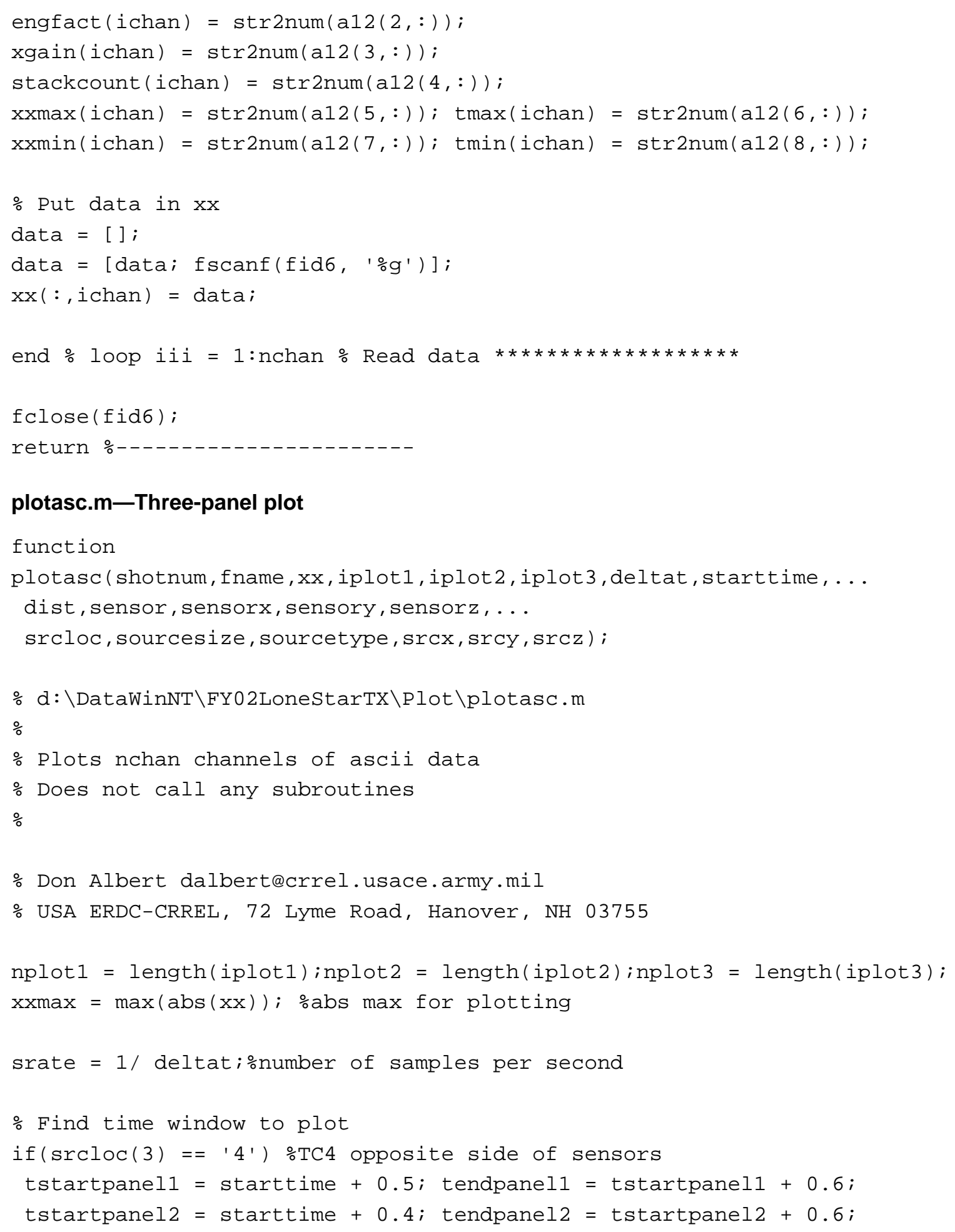




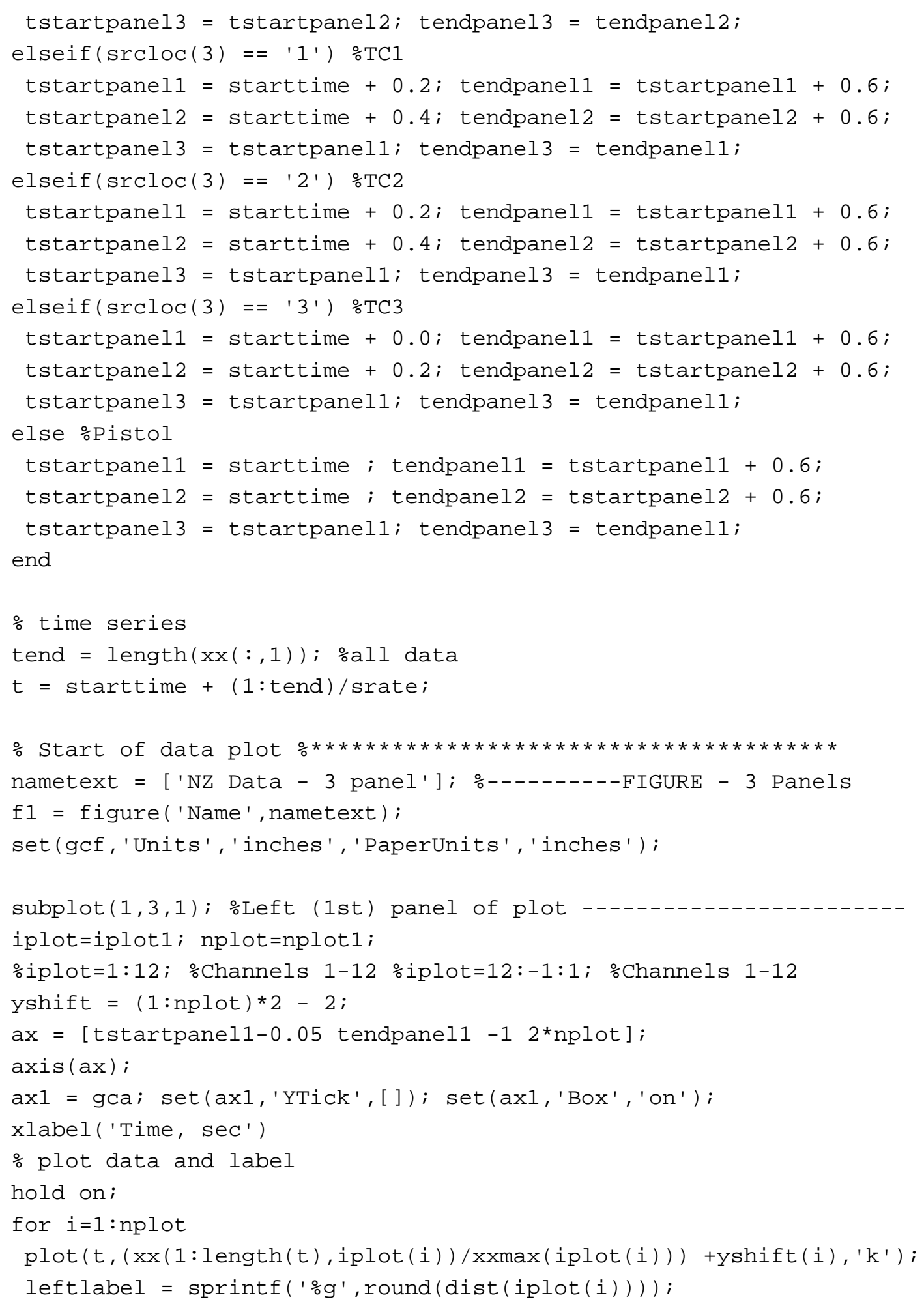




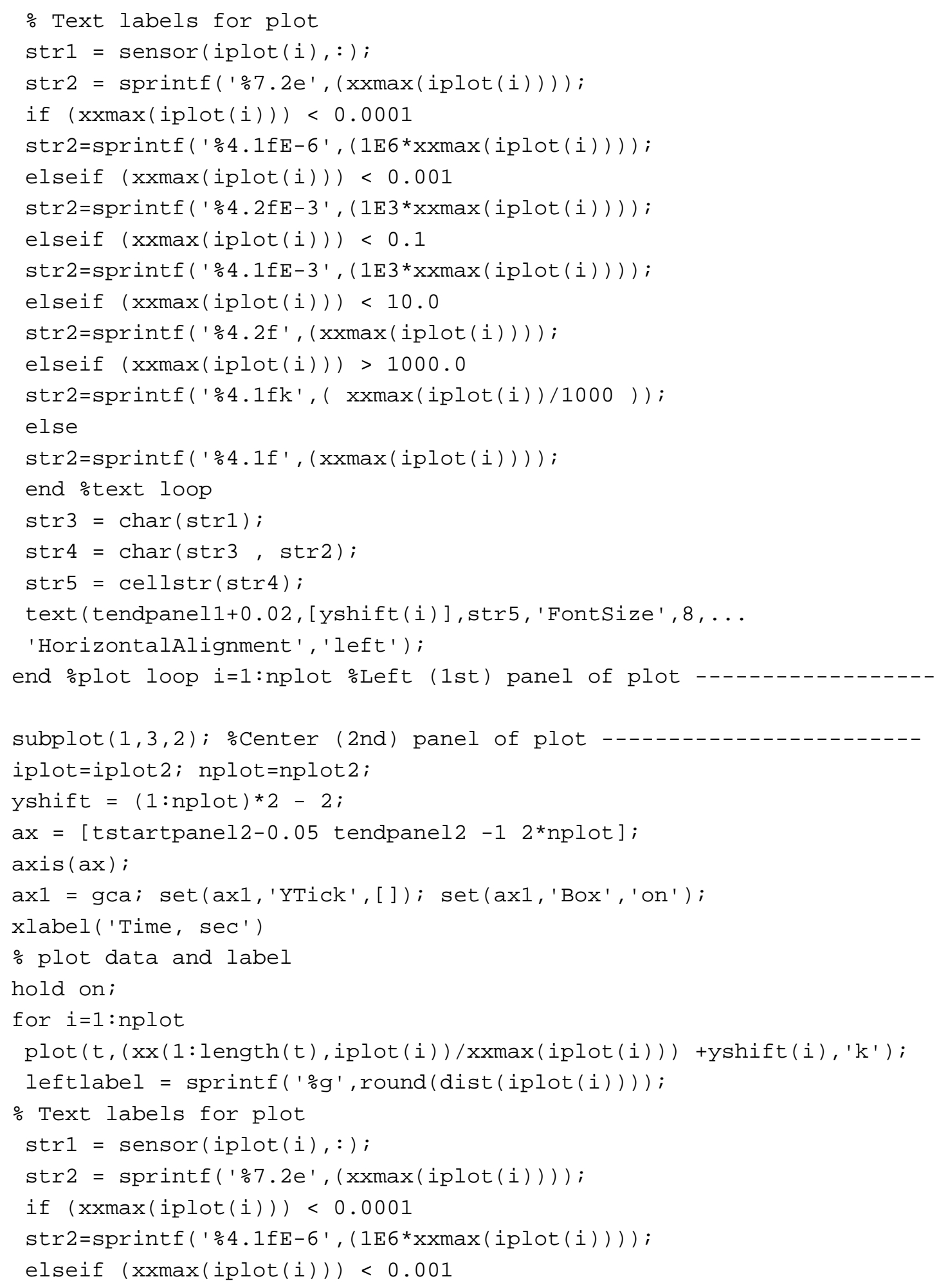




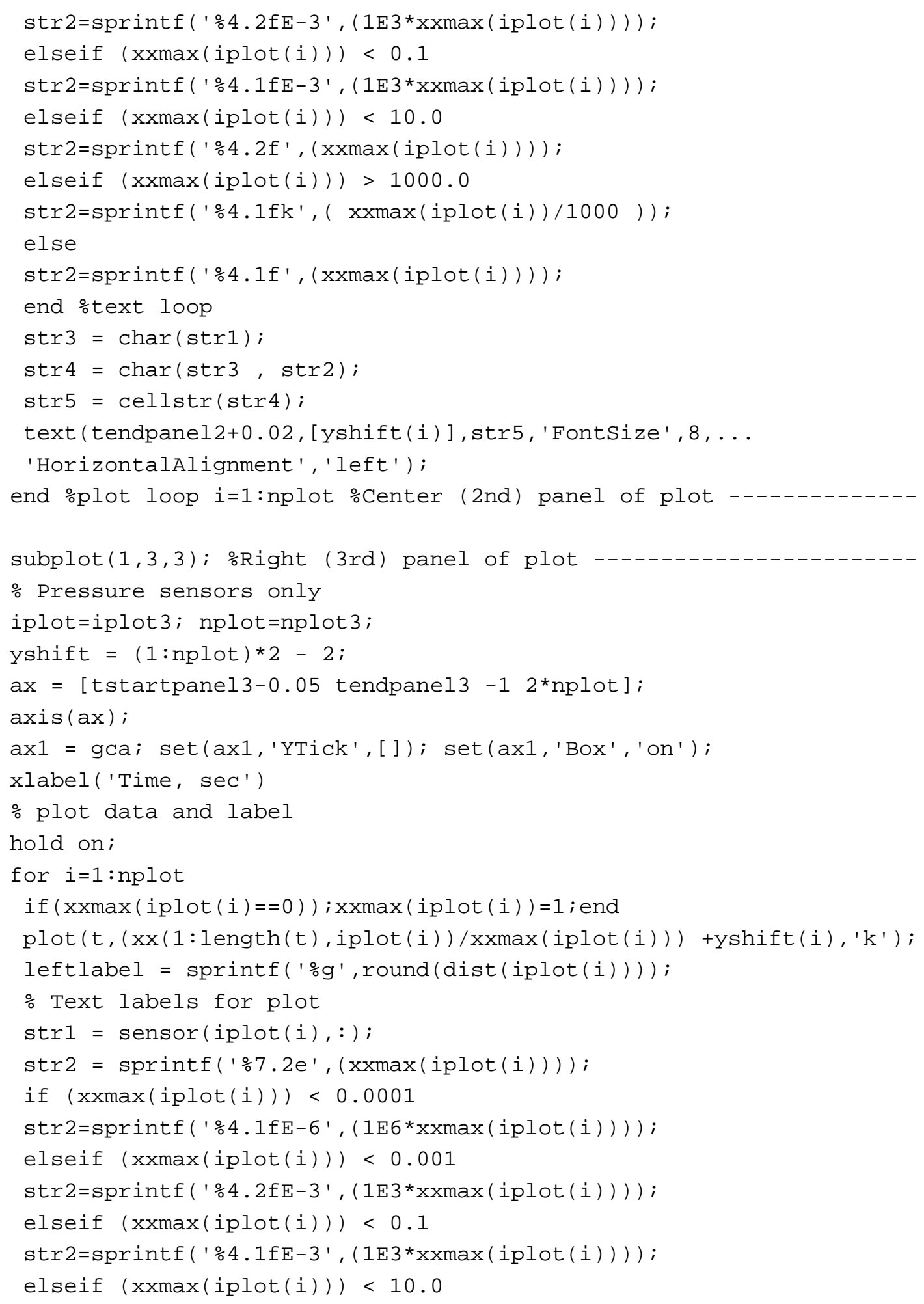




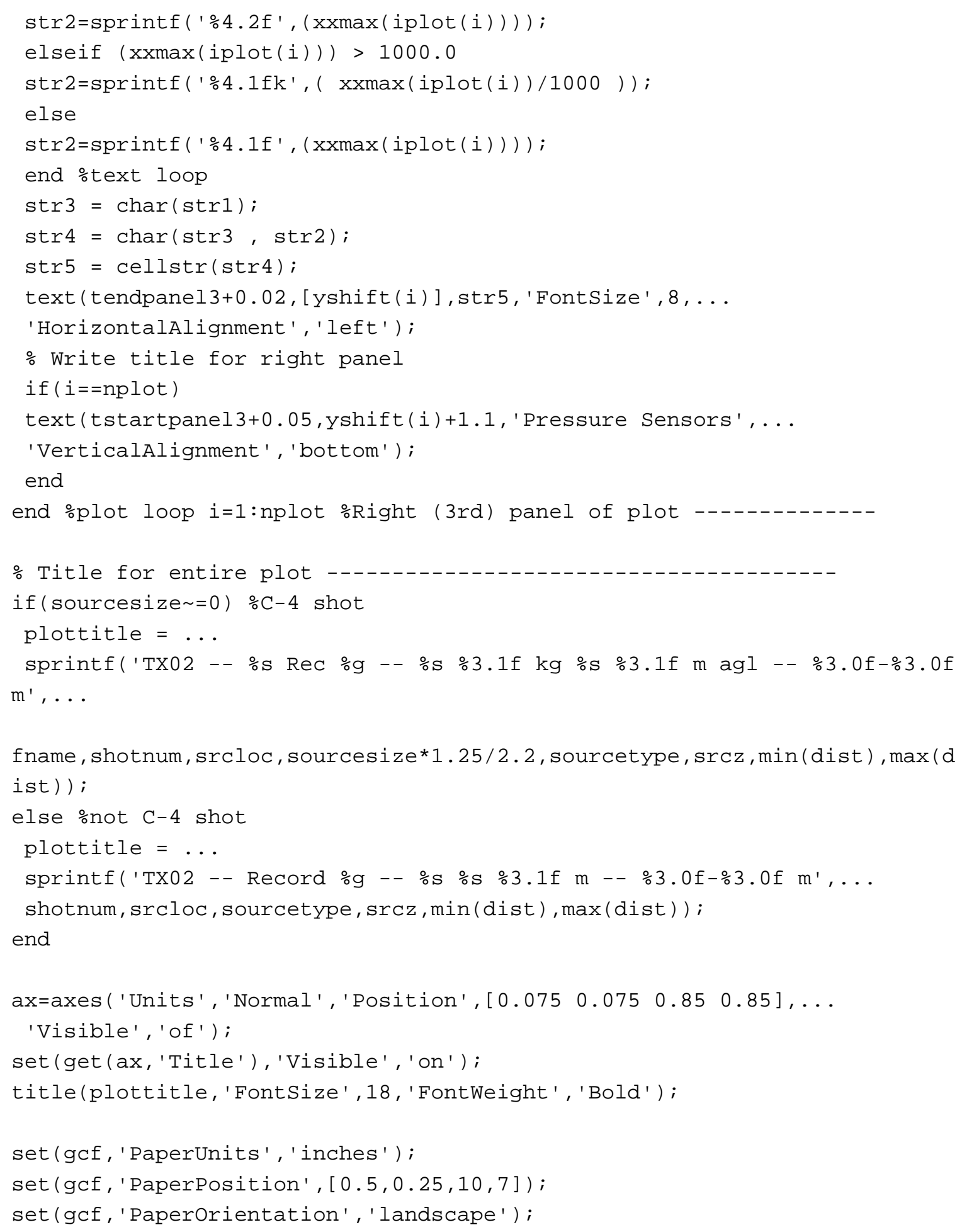


return 


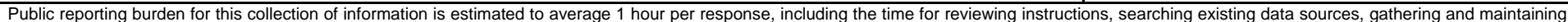

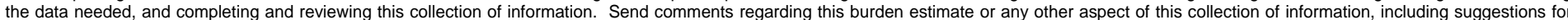

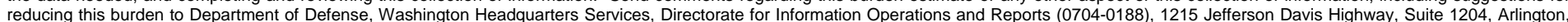

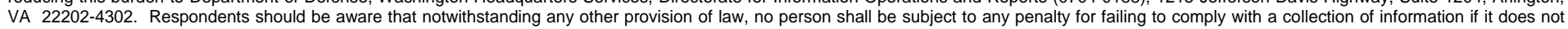
display a currently valid OMB control number. PLEASE DO NOT RETURN YOUR FORM TO THE ABOVE ADDRESS.
1. REPORT DATE $(D D-M M-Y Y Y Y)$ 2. REPORT TYPE
May 2005
3. DATES COVERED (From - To)

4. TITLE AND SUBTITLE

Short-range Seismic and Acoustic Signature Measurements Through Forests

5a. CONTRACT NUMBER

5b. GRANT NUMBER

5c. PROGRAM ELEMENT NUMBER

6. AUTHOR(S)

5d. PROJECT NUMBER

Stephen N. Decato, Donald G. Albert, Frank E. Perron Jr., and David L. Carbee

5e. TASK NUMBER

5f. WORK UNIT NUMBER

7. PERFORMING ORGANIZATION NAME(S) AND ADDRESS(ES)

8. PERFORMING ORGANIZATION REPORT NUMBER

Cold Regions Research and Engineering Laboratory

72 Lyme Road

ERDC/CRREL TR-05-10

Hanover, NH 03755-1290

9. SPONSORING I MONITORING AGENCY NAME(S) AND ADDRESS(ES)

10. SPONSOR/MONITOR'S ACRONYM(S)

U.S. Army Corps of Engineers

Washington, DC 20314-1000

11. SPONSOR/MONITOR'S REPORT NUMBER(S)

\section{DISTRIBUTION / AVAILABILITY STATEMENT}

Approved for public release; distribution is unlimited

13. SUPPLEMENTARY NOTES

\section{ABSTRACT}

The effect of forests on low frequency military noise propagation is unknown. As part of a joint project, ERDC-CERL and ERDC CRREL conducted measurements at the Lone Star Army Ammunition Plant located in Texarkana, Texas, to investigate these effects. In this report, the short-range measurements conducted by ERDC-CRREL are documented. Blast noise waveforms produced by C4 explosions at distances from 30 to $567 \mathrm{~m}$ were recorded and are presented in this report. In all, 42 different explosions were recorded, producing 314 high quality pressure waveforms for analysis. Additional reports documenting the long-range measurements and analyzing the recorded data are in preparation.

\section{SUBJECT TERMS}

Acoustics

Acoustics measured through forest 16. SECURITY CLASSIFICATION OF:

\section{a. REPORT}

Unclassified

\section{b. ABSTRACT}

Unclassified
Blast noise

Blast waves

Forest effects

c. THIS PAGE

Unclassified

Sound propagation

Trees

Vegetation

17. LIMITATION OF ABSTRACT

Unclassified
18. NUMBER OF PAGES

135 19a. NAME OF RESPONSIBLE PERSON

19b. TELEPHONE NUMBER (include area code) 
